\section{$18 /, 1,46095$ \\ SANDIA REPORT}

SAND96-1497 • UC-404

Unlimited Release

Printed July 1996

\title{
Template-Mediated Ontogenesis: A Novel Approach to Mesomorphic Materials
}

J. E. Martin, M. T. Anderson, J. Odinek, P. Newcomer

Prepared by

Sandia National Laboratories

Albuquerque, New Mexico 87185 and Livermore, Califomia 94550

for the United States Department of Energy

under Contract DE-AC04-94AL85000

Approved for public release; distribution is unlimited.

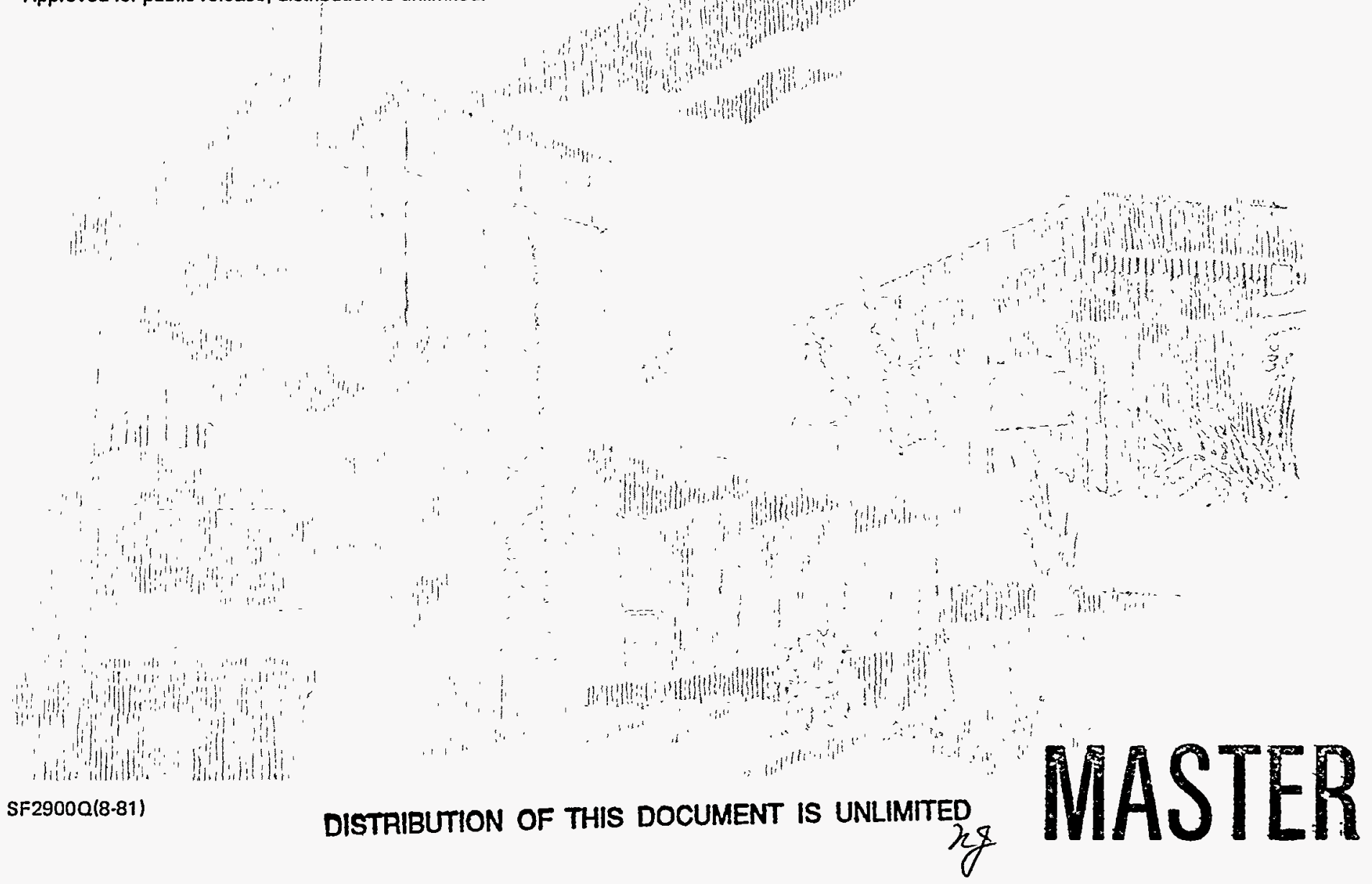


Issued by Sandia National Laboratories, operated for the United States Department of Energy by Sandia Corporation.

NOTICE: This report was prepared as an account of work sponsored by an agency of the United States Government. Neither the United States Government nor any agency thereof, nor any of their employees, nor any of their contractors, subcontractors, or their employees, makes any warranty, express or implied, or assumes any legal liability or responsibility for the accuracy, completeness, or usefulness of any information, apparatus, product, or process disclosed, or represents that its use would not infringe privately owned rights. Reference herein to any specific commercial product, process, or service by trade name, trademark, manufacturer, or otherwise, does not necessarily constitute or imply its endorsement, recommendation, or favoring by the United States Government, any agency thereof or any of their contractors or subcontractors. The views and opinions expressed herein do not necessarily state or reflect those of the United States Government, any agency thereof or any of their contractors.

Printed in the United States of America. This report has been reproduced directly from the best available copy.

Available to DOE and DOE contractors from

Office of Scientific and Technical Information

PO Box 62

Oak Ridge, TN 37831

Prices available from (615) 576-8401, FTS 626-8401

Available to the public from

National Technical Information Service

US Department of Commerce

5285 Port Royal Rd

Springfield, VA 22161

NTIS price codes

Printed copy: A06

Microfiche copy: A01 
SAND96-1497

Distribution

Unlimited Release

Category UC -404

Printed July 1996

\title{
Template-Mediated Ontogenesis: A Novel Approach to Mesomorphic Materials

\author{
J.E. Martin, M.T. Anderson, J. Odinek, P. Newcomer \\ Nanostructures and Advanced Materials Chemistry \\ Sandia National Laboratories \\ Albuquerque, NM 87185
}

\begin{abstract}
In this report we describe the methods we have developed for producing stable periodic mesoporous silica gels, thin films of mesoporous silica for sensor applications, a route to nonaqueous synthesis, and the use of various additives in controlling the pore size and structure of these materials. Mesoporous silica is formed by templating silica precursors around micelles of cationic quaternary ammonium surfactants. During the synthesis these micelles undergo a phase transition to a hexagonal, lamellar or cubic liquid crystalline state, thus imposing periodic order on the amorphous silica which occupies the interface of the hydrophilic cationic headgroups of the surfactants. The product of the bulk wet synthesis is a gel composed of micron size silica/surfactant particles, each of which consists of one or more crystalline domains of silica condensed around the surfactant template. The wet gel can then be washed and pyrolized to remove the surfactant template, yielding the periodic mesoporous silica product.
\end{abstract}




\section{Table of Contents}

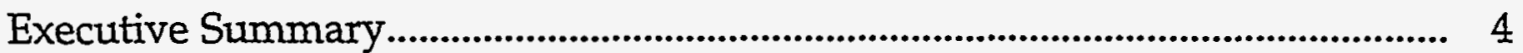

I. Background and Motivation.......................................................................... 6

II. Synthesis of Periodic Silica from Molecular Precursors in Methanol... 8

A. Epitome..................................................................................................... 8

B. Introduction................................................................................................ 9

C. Experimental.......................................................................................... 10

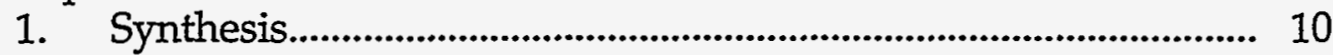

2. X-ray Diffraction................................................................................ 11

3. Light Scattering................................................................................. 12

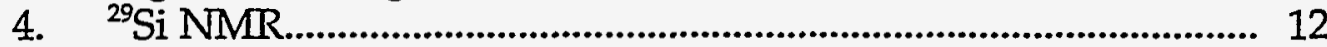

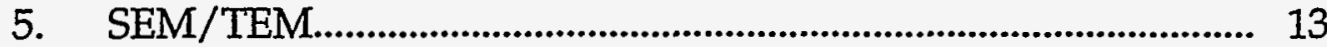

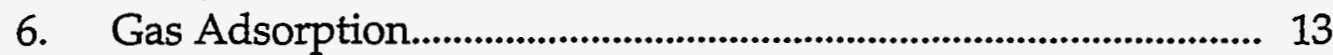

D. Results and Discussion....................................................................... 13

1. Formation of Homogeneous Solutions with Methanol........ 13

2. Effect of Methanol on Micelle Formation.:............................... 14

3. Effect of Ionic Strength on Micelle Formation.......................... 18

4. Micellization and Product Formation Mechanism.................. 20

5. Effect of Methanol on Yield.......................................................... 24

6. Effect of TMOS and Methanol on Kinetics................................ 25

7. Effect of TMOS and Methanol on Yield..................................... 26

8. Effect of TMOS and Methanol on Framework Condensation.28

9. Effect of Methanol on Micelle Radius and Pore Diameter..... 30

10. Controlling Porosity......................................................................... 30

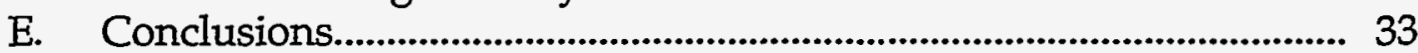

III. Synthesis of Periodic Mesoporous Thin Films.............................................. 34

A. Epitome..................................................................................................... 34

B. Introduction.............................................................................................. 35

C. Experimental......................................................................................... 36

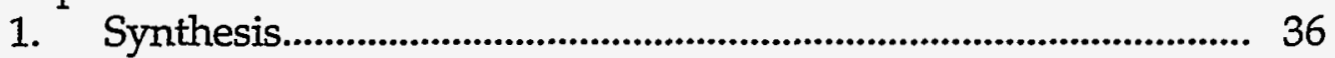

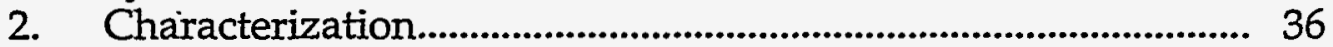

3. Gas Adsorption........................................................................... 37

D. Results and Discussion........................................................................ 37

1. Solution Homogeneity and Reaction Kinetics.......................... 37

2. Spin Coating Premixed Water:Cosolvent Solutions................ 39

3. Gas-Catalyzed Thin Film Synthesis.............................................. 45

4. Optimizing Film Thickness and Coverage.................................. 53

5. Microstructure and Sorptive Properties.................................... 55

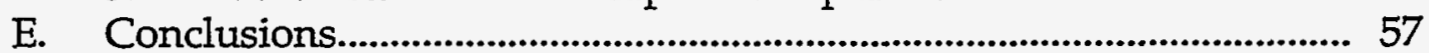

IV. Monolithic Periodic Mesoporous Silica Gels............................................... 58

A. Epitome.................................................................................................... 58

B. Introduction........................................................................................ 59

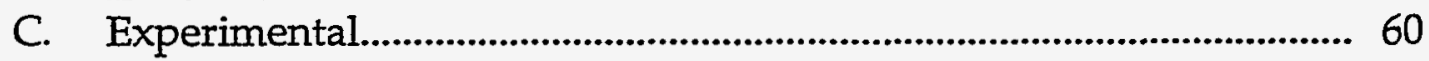

1. Synthesis........................................................................................ 60 


\section{DISCLAIMER}

Portions of this document may be illegible in electronic image products. Images are produced from the best available original document. 


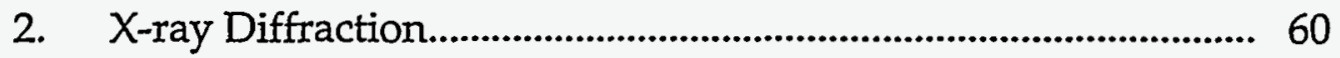

3. Small Angle X-ray Scattering........................................................... 61

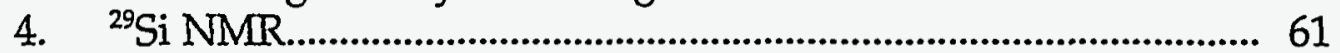

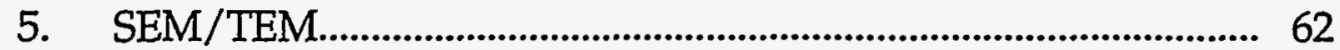

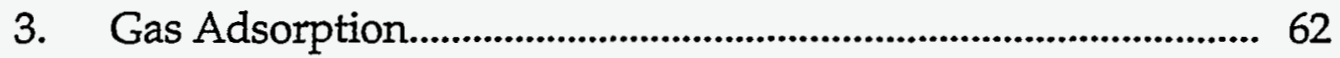

D. Results and Discussion........................................................................ 62

1. Silica Source: The Key to Forming Monolithic PMSGs......... 62

2. Structure of PMSGs.................................................................... 63

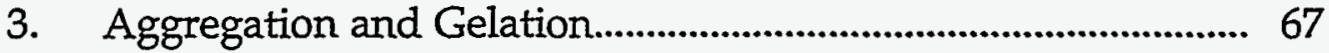

4. Controlling the Interparticle Density........................................ 72

5. Accessing Intraparticle Porosity..................................................... 76

6. Measuring Inter- and Intraparticle Porosity.............................. 78

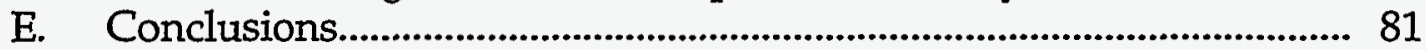

V. Effects of Cosolvents, Swelling Agents and Added Salt............................ 82

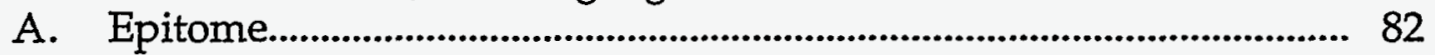

B. Introduction.............................................................................................. 82

C. Experimental........................................................................................... 85

1. Synthesis........................................................................................... 85

2. X-ray Diffraction............................................................................... 86

3. SEM/TEM................................................................................... 87

4. Gas Adsorption.............................................................................. 87

D. Results and Discussion..................................................................... 87

1. Cosolvents and the Hexagonal Existence Region.................... 87

2. Nonaqueous Synthesis.................................................................. 100

3. Cosolvents, Micellization and Product Periodicity................. 103

4. Pore Size Control......................................................................... 106

5. Mixed-Metal Frameworks........................................................ 107

6. Effect of Cosolvents on Microstructure.................................. 110

7. Swelling Agents.............................................................................. 110

8. Added Salt....................................................................................... 115

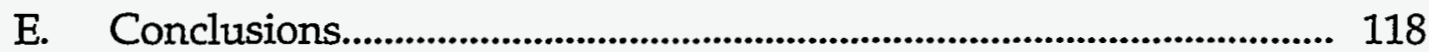

Acknowledgements................................................................................................. 118

References.................................................................................................................. 119 


\section{Executive Summary}

We have developed techniques to synthesize periodic mesoporous silica (PMS) in the form of gels and thin films. Mesoporous materials are in many ways similar to zeolites, except that the pore sizes are much larger, on the order of $30 \AA$ : Zeolites are synthesized by templating silica and alumina precursors around quaternary ammonium salts of typical molecular dimensions, whereas PMSs are synthesized by templating silica precursors around micelles of cationic quaternary ammonium surfactants. During the synthesis these micelles undergo a phase transition to a hexagonal, lamellar or cubic liquid crystalline state, thus imposing periodic order on the amorphous silica which occupies the interface of the hydrophilic cationic headgroups of the surfactants. The product of the bulk wet synthesis is a gel composed of micron size silica/surfactant particles, each of which consists of one or more crystalline domains of silica condensed around the surfactant template. The wet gel can then be washed and pyrolized to remove the surfactant template, yielding the periodic mesoporous silica product. Within the pores of the silica framework uncondensed hydroxl groups are now exposed that can be chemically functionalized to yield materials with high specificity for the binding of chemicals, and this is useful for selective filtration and catalysis.

In this report we describe the methods we developed for producing stable mesoporous silica gels, thin films for sensor applications, a route to nonaqueous synthesis, and the use of various additives in controlling the pore size and structure of these materials. We are now actively pursuing applications of these new materials, including coatings for surface acoustical wave sensors for automotive applications.

The first work we discuss is the effect of the solution thermodynamics on the formation of PMSs. Of course, the solution thermodynamics is critical to the formation of the liquid crystalline template, and it is well know that template formation is favored by a hydrophilic environment. However, the 
silica precursor we use is highly hydrophobic until it becomes at least partially hydrolyzed, so we were faced with a miscibility issue that affected the quality and yield of the product. We found that we could add methanol as a cosolvent to solubilize the precursors, but that this affected micellization by increasing the critical micelle concentration (decreasing the surfactant in the form of micelles). Fortunately, we found that adding controlled amounts of methanol allowed us to quickly solubilize the precusors yet still form the liquid crystalline template, yielding a high quality PMS with a large domain size.

The second result we discuss is the development of mesoporous silica gels. We were able to produce gels by using cosolvents to control the solution thermodynamics and by using a molecular silica precursor that hydrolyzes very quickly. These gels, which can be produced in a few seconds, are stable to drying and we are now investigating methods of solvent extraction that would prevent significant volume change, such as super critical fluid extraction, to yield a mesoporous aerogel. We are pursuing a patent on this work.

We next discusss the issue of synthesizing mesoporous silica thin films, which we found could be accomplished using an approach we call gas-phase catalysis. In this method the hydrolysis of molecular silica precursors is base catalyzed after the wet film is formed by admitting ammonia gas into a reaction chamber. The gas catalyses the silica precursors into multidentate silanols, which then form the mesoporous material in situ. We are pursuing a patent on this work as well.

Finally, we investigated the effect of a large number of cosolvents, swelling agents, and added salts on the properties of the PMGs. These investigations led to a substantially nonaqueous synthesis of these materials, useful for thin film production and other applications. The swelling agents enabled us to produce lamellar products with greatly increased lattice parameters, but we were not able to make a really good hexaganol product. 


\section{Background and Motivation}

Mesomorphic materials have properties that are fundamentally different than those of their macroscopic constituents. This frequently leads to greater utility, but producing microheterogeneous materials is often difficult since the only constraint imposed during synthesis is macroscopic- the reaction bath itself. In this proposal we will use surfactant templates to produce novel microheterogeneous materials whose unique properties address specific materials goals. These include chemically selective filters and coatings for surface acoustical wave devices.

Surfactants make ideal mesoscale templates due to the variety of morphologies they assume in solution and because of the ease with which the characteristic size can be controlled. Depending on the concentration, surfactants can form thermodynamically stable spherical, ellipitical, cylindrical or lamellar structures, and even complex bicontinuous phases whose interface is thought to be a Schwartz surface - a family of surfaces where every point is a saddle point. More complex structures can be made by driving the system out of equilibrium, to create unstable interpenetrating spinodal structures. In any case, our general scheme is to mix the surfactants with a hydrophilic and hydrophobic phase, at least one of which is reactive, in order to coerce the reactive component to assume the morphology of the template. This approach should be successful whenever the chemical reactions do not alter the phase behavior of the surfactant. The spherical, ellipitical, cylindrical and lamellar templates will give rise to particles with the corresponding morphologies, whereas the bicontinuous and spinodal templates will yield microheterogenous materials with high fracture toughness.

In special circumstances, such as in the nucleation and growth of colloids, continuous phase synthesis may naturally lead to mesoscale structures. However, it is generally essential to impose a template upon the reacting species. For example, a recent issue of Scientific American featured the synthesis of solid acid catalysts in zeolitic cages. In this case, synthesis in a 
confining medium proved to be the key to producing a catalyst with high specificity. Likewise, a recently developed, Sandia-patented process produces nanometer-size metal particles by confining the reaction to an inverse spherical micellar template. These metal clusters have been demonstrated to be extraordinary hydrogenation catalysts, due to the high density of defect sites on their highly curved surfaces. These nascent attempts at template-mediated synthesis show promise for this to be a methodology that will yield a new generation of materials.

In the studies that follow, we have mixed molecular silica precursors with cationic quaternary ammonia salts at high $\mathrm{pH}$ to produce lamellar and hexagonal porous silica structures. The liquid crystalline surfactant template actually forms during the reaction, the phase transition from a micellar phase being driven by the production of multidentate silica ligands produced from the hydrolysis of silica alkoxides. The periodicity in these materials is $\sim 40-55 \AA$ and the pore size in the hexagonal phase is $\sim 25-30 \AA$. Zeolites have pore sizes generally smaller than $8 \AA$ so these materials fill a size range that cannot be achieved by other techniques. After the silica-surfactant system assembles into the liquid crystalline phase, ( $\sim \mathrm{s}$ at ambient temperature) condensation of the silica occurs to form a nearly fully bonded framework in a few minutes. The material can then be treated by a number of techniques to effect removal of the surfactant template from the silica phase. A silica framework remains that has a significant number of unresolved siloxyl groups within the pores. These groups can easily be functionalized to achieve a desired chemical specificity or catalytic activity. For example, reacting with trimethoxysilanol renders the pore volume hydrophobic, thus encouraging hydrocarbon absorption.

In the following we will discuss many of the synthetic approaches we have developed to make bulk mesoporous materials and thin films. These studies have focused on both understanding the factors that direct structure and on making tailorable materials. 
II. Synthesis of Surfactant-Templated Periodic Silica from Molecular Precursors in Water: Methanol Solutions

\section{A. Epitome}

Using the molecular silica source tetramethoxysilane (TMOS) and its parent alcohol, methanol, along with surfactant, base, and water we are able to synthesize periodic mesoporous silica (PMS) from homogeneous solutions. We varied $r$ ( $w t \%$ methanol in aqueous solution) from $0 \%$ to $100 \%$ to determine its effect on solution homogeneity, micellization, formation mechanism, long-range order, yield, microstructure, framework condensation, and pore diameter of the products. We find four regions of behavior: In Region I ( $r=0$ to $\sim 25 \%$ ) the TMOS is not completely solubilized prior to gel formation, so the solutions are not homogeneous; however the concentration of micelles is highest in this region so PMS forms, but with less than optimal yields and long-range order. In Region $\Pi(r=\sim 25$ to $\sim 60 \%)$ the solutions are homogeneous and micelles form, which leads to PMS with optimal long-range order and in high yield. In Region III ( $r=\sim 60$ to $90 \%$ ) the solutions are homogeneous, but micelles do not form, and PMS with short-range order forms in less than optimal yield. In region IV ( $x>\sim 90 \%$ ) solutions are homogeneous, micelles do not form, and the only products are amorphous silica.

In general our cosolvent-based molecular approach to PMS leads to the rapid formation of weak particulate gels that consist of submicron particles. After template removal, apparent BET surface areas can exceed $950 \mathrm{~m}^{2} / \mathrm{g}$ and the void volume can exceed $0.6 \mathrm{cc} / \mathrm{g}$. There is a high fraction of uncondensed silica in the as-made product $\left(Q^{3} / Q^{4} » 2.1\right)$, but after calcination a strong, bonded siloxane framework forms $\left(Q^{3} / Q^{4} » 0.4\right)$. 


\section{B. Introduction}

Periodic mesoporous silica can be synthesized by assembling an inorganic phase around organic surfactants. [1-6] These templated materials are unique in that they have unimodal, narrow pore size distributions with pore sizes tunable from $\sim 15$ to $\sim 100 \AA$. A wide variety of uses for periodic mesophases have been demonstrated or envisioned, including acid catalysts [7-9] (although acidity is less than for zeolites), [6,10] oxidative catalysts for bulky organics, [11-13] supports for noble-metal oxidation and hydrogenation catalysts, [14] supports for NiMo in hydrocracking, [15] and supports for heteropolyacid ion catalysts, $[16,17]$ hosts for conducting polyaniline filaments [18] and carbon wires, [19] separations media (especially in membrane form), coatings for sensors, [20] and high surface area adsorbents. [1]

To synthesize periodic mesoporous silica four-reagents are required: a silica source, water, a solvent, a catalyst, and a surfactant. In a typical synthesis, a silica source is added to a basic aqueous solution of a micelleforming cationic surfactant. The silica source is hydrolyzed and condensed to form multidentate, multicharged anions that can coordinate with the surfactant headgroups. The strong interaction between the silica species and the surfactants leads to phase separation of the silica/surfactant arrays into microdomains that have an ordered, liquid crystalline structure. The domains aggregate and agglomerate to form colloidal particles that eventually precipitate. Over an extended period of time, the silica condenses to form a bonded siloxane framework with the evolution of much heat. The surfactant templates can then be removed by one of several processes to form the porous material. In these cases the PMS is made from inhomogeneous solutions.

For applications it is important to have a high yield of pure materials; this maximizes the specific activity or surface area of the products, eliminates unwanted byproducts, and makes the synthetic process most cost effective. We realized that to achieve nearly quantitative yields, materials should be made from a stoichiometric mixture of reagents in homogeneous solutions. 
To do this we need molecular precursors (i.e. alkoxides) and a solvent that has a low enough dielectric constant to rapidly solubilize the hydrophobic silicon alkoxide, but a high enough dielectric constant so that the surfactant acts as a chemical dipole. To achieve homogeneous solutions we thus use a mixed water/alcohol system and tetramethoxysilane (TMOS). The lower dielectric constant methanol acts as an homogenizer for the alkoxide but still allows micelle formation over a wide water:methanol range. In fact, by varying the concentration of methanol in the solution the extent of micellization in the precursor solution can be contiguously varied. This allows us to investigate the effects of micellization on product structure and long-range order.

We find that synthesis of surfactant-templated mesophases from homogeneous solutions with TMOS as the silica source leads to important and interesting differences compared to products made from inhomogeneous solutions; these differences include: an essentially quantitative yield of product with respect to silicon and surfactant; a significant effect on the tertiary structure of the product, giving gels rather than precipitates; dramatically reduction in the time to make an ordered product $(<10 \mathrm{~s})$; pore diameters that can be continuously tailored over a $\sim 5 \AA$ range; as-made silica/surfactant composites that have a high fraction of uncondensed silica species (after calcination the extent of framework condensation is virtually identical to that for materials made hydrothermally or from TEOS); and materials in three useful forms: i.e. thin films, monoliths of arbitrary shape and size, and powders of submicron particles. Here we concentrate on the effects of methanol and TMOS on the spatial, kinetic, and adsorptive properties of powdered PMS.

\section{Experimental}

C.1 Synthesis - Our work focused on 1-d periodic hexagonal mesoporous silica. A typical synthesis involved mixing: $9.8 \mathrm{~g}$ deionized water/methanol, 
$0.2 \mathrm{~g}(0.55 \mathrm{mmol})$ of cetyltrimethylammonium bromide, $\mathrm{C}_{19} \mathrm{H}_{42} \mathrm{NBr}$, $\left(\mathrm{C}_{16} \mathrm{TMABr}\right.$; Fisher, $\left.99+\%\right), 65 \mathrm{~mL} 50 \mathrm{wt} \% \mathrm{NaOH}(1.14 \mathrm{mmol})$, and $0.625 \mathrm{~mL}$ ( $4.23 \mathrm{mmol}$ ) tetramethoxysilane (TMOS, United Chemical Technologies or Aldrich). Molar ratios of $\mathrm{C}_{16} \mathrm{TMABr}: \mathrm{NaOH}$ :TMOS in the initial mixture were 0.13:0.27:1. The water to methanol weight ratio (expressed as $r=w t \%$ methanol) was varied from $\mathrm{r}=0 \%$ to $100 \%$. The $\mathrm{H}_{2} \mathrm{O}$ :Si ratio ranged from 129 to $<1$.

The optimal synthetic method was to inject $4.2 \mathrm{mmol}$ of TMOS into 10 g of a rapidly stirred alkaline $\mathrm{C}_{16} \mathrm{TMABr}(2 \mathrm{wt} \%)$ water:methanol $(75: 25 \mathrm{w} / \mathrm{w})$ solution. The mixture turns translucent, then transparent, and then abruptly opaque as a white particulate gel forms. After the gel forms the sample continues to generate large amounts of heat.

After aging ( $1 \mathrm{~min}$ to $30 \mathrm{~d}$ ) the wet silica/surfactant gels were suction filtered, washed with deionized water and/or methanol and dried in air. The intraparticle surfactant typically was removed from the product by calcination. The calcination process involved a linear temperature ramp from 25 to $550^{\circ} \mathrm{C}$ over $2-10 \mathrm{~h}$ flowing $\mathrm{N}_{2}$; an isotherm at $550{ }^{\circ} \mathrm{C}$ for $2 \mathrm{~h}$; cooling to $\left\langle 300{ }^{\circ} \mathrm{C}\right.$; a linear ramp to $550^{\circ} \mathrm{C}$ in flowing $\mathrm{O}_{2}$; an isotherm for $>10$ $\mathrm{h}$; and cooling to room temperature in flowing oxygen (furnace off). The calcined product was usually white with a small amount of black carbonaceous material; if the product was not white it was again calcined in $\mathrm{O}_{2}$ at $550^{\circ} \mathrm{C}$ for $» 10 \mathrm{~h}$.

C.2 X-ray Diffraction - Data were collected with a Scintag PAD V instrument using nickel-filtered $\mathrm{Cu} \mathrm{K}_{\mathrm{a}}$ radiation. Data were collected in continuous scan mode from 1.5 to $10^{\circ} 2 \theta$ with a $0.02^{\circ}$ sampling interval and a $1^{\circ} / \mathrm{min}$ scan rate. Slits widths starting from the source were $1,2,1$, and $0.3 \mathrm{~mm}$. Tube voltage was $45 \mathrm{kV}$ and tube current was $35 \mathrm{~mA}$. Peak positions and full-widths at half height were determined with Scintag analysis software (TC9 package). Peak positions for the periodic hexagonal phase were corrected with an external 
standard routine. The routine used four Bragg peaks $(100,110,200,210)$ for the correction.

C.3 Light Scattering - Dynamic and static light scattering measurements were made with a $63 \mathrm{~mW}$ NEC He-Ne laser, using a 256 channel Langley-Ford correlator. A Malvern index-matching temperature- controlled scattering vat, a Malvern detector assembly, using an RCA FW130 photomultiplier tube, and an Aerotech 12 inch stepper-motor-driven goniometer complete the basic light scattering hardware. The system is automated by a DEC PDP-11/73b computer.

Intensity autocorrelation functions $S_{2}(q, t)=\langle I(q, 0) I(q, t)>$ were collected and the Seigert relation was used to obtain the field autocorrelation function $S_{1}(q, t)=<E(q, 0) E(q, t)>$ from these. [21] The field autocorrelation function data were fit to single exponential decays and the micelle -radius was determined from the heterodyne decay rate $\Gamma$ from the standard relation $\Gamma=D_{t} q^{2}$, where $q$ is the scattering wavevector and $D_{t}$ is the translational diffusion constant. The Stokes-Einstein relation $D_{t}=k_{B} T / 6 \pi \eta R$ was then used to extract the radius, where $\eta$ is the solvent viscosity.

The static intensity data were taken on the same instrument using a statistical procedure to discard anomalously high intensity readings due to dust. Filtration of all micelle samples in a clean bench reduced dust contamination.

C.4 ${ }^{29}$ Si NMR - High resolution, magic angle spinning spectra were recorded on a Chemagnetics instrument equipped with a 4.7 Tesla magnet. Powder samples were packed into $2.5 \times 6 \mathrm{~cm} \mathrm{ZrO}_{2}$ rotors. Spectra were recorded at a resonance frequency of $39.7 \mathrm{MHz}$, with a $7.25 \mathrm{~ms}$ pulse at $90_{\mathrm{i}}$, and a pulse delay of $120 \mathrm{~s}$. Final spectra are an average of 512 scans. Tetramethylsilane (TMS) was used as a standard to define $0 \mathrm{ppm}$. Data were 
analyzed and integrated with MacNMR software routines from Tecmag to determine $Q$ ratios and shifts relative to TMS.

C.5 SEM/TEM - A JOEL 1200EX transmission electron microscope (TEM) with ASID (SEM) attachment was used to observe the microstructure and grain size of the powder samples. In order to observe individual grains, the aggregated powders were ground under methanol in a mortar and pestle. The suspended powder was caught on the holey carbon film of a $3 \mathrm{~mm}$ copper grid. Bright field TEM or diffraction contrast imaging was done at $120 \mathrm{kV}$ and involved low (20k times) and high (300k times) magnifications of the individual grains and small aggregates. SEM micrographs were obtained that reveled the morphology of powder aggregates and the size of the grains.

C.6 Gas Adsorption - A Micromeritics ASAP 2000 was used to collect isothermal $\mathrm{N}_{2}$ adsorption data at $77 \mathrm{~K}$. Samples were degased overnight at $200{ }^{\circ} \mathrm{C}$. 4-point BET analyses were performed to determine the apparent surface areas. Pore diameters were determined by application of the Kelvin Equation to the desorption branch of the data.

\section{Results and Discussion}

D.1 Formation of. Homogeneous Solutions with Water:Methanol -- In order to optimize yield we sought to make periodic mesoporous silica from homogenous solutions. In previous work, $[1,2,6]$ periodic mesoporous silica has been made from non-molecular silica sources, such as, fumed silica, precipitated silica, or water glass, but the solutions are inhomogeneous and yield, especially with respect to surfactant, is low.

PMS has also been synthesized from the molecular silica source tetraethoxysilane (TEOS) with water as the solvent; [3,4] however, owing to the hydrophobic nature of TEOS, an emulsion forms that eventually leads to the production of complex amorphous silica byproducts and PMS. We have 
directly observed these siliceous products with optical microscopy. The hydrolyzed TEOS forms micron size seed-pod like structures that eventually burst and expel partially hydrolyzed silicate species; the shell remains and ends up mixed with PMS.

A well documented method to overcome the insolubility of silicon alkoxides in aqueous solutions is to add a homogenizing agent. [22] In our syntheses we add a small amount of the alcohol methanol, to the readily hydrolyzable, molecular silica source, tetramethoxysilane (TMOS). The dielectric constant of methanol is lower than water so the mixture readily dissolves the hydrophobic alkoxide. The kinetics of product formation are so rapid that a portion of the TMOS remains emulsified at the gel point $\left(t_{\mathrm{gel}} \equiv\right.$ time at which the mesoporous product gels) until methanol weight ratio in the mixed solvent exceeds $\sim 25 \%$ (referred to as $r=25 \%$ ). At this ratio the TMOS is solubilized before the mesoporous product forms (i.e. $t_{\text {solubility }}<t_{\text {gee }}$ ) and the product forms out of a homogenous solution.

D.2 Effect of Methanol on Micelle Formation - Adding methanol to the precursor solution is expected to change the extent of micellization by changing the effective dielectric constant of the solvent. To quantify the changes in micellization in the $\mathrm{pH} 13.2$ precursor solution we applied static and dynamic light scattering in two ways.

In the first method we simply took advantage of the fact that the scattering intensity above the solvent background $\Delta \mathrm{I}(\mathrm{q})$ from a micelle solution [23] is

$$
\Delta \mathrm{I}(\mathrm{q})=\mathrm{bc} \mathrm{c} M S(\mathrm{q}) f(\mathrm{qR})
$$

Here $\mathrm{M}=\mathrm{nm}$ is the micelle mass, $\mathrm{n}$ is the number of the surfactant molecules per micelle, $m$ is the mass of a surfactant molecule, $c^{\prime}$ is the concentration of surfactant in the form of micelles, $R$ is the radius of gyration of a micelle, 
$f(q R)$ is the micelle form factor (conveniently expressed by a Bessel function) that gives the scattering from a single micelle, $S(q)$ is the structure factor that gives the optical interference that arises from correlations between micelles, which in turn are due to interactions between micelles. Finally, the scattering wavevector $\mathrm{q}=4 \pi \sin (\theta / 2) / \lambda$, in terms of the scattering angle $\theta$ and the wavelength in the scattering medium $\lambda(633 \mathrm{~nm} / 1.33)$, and $\mathrm{b}$ is a constant that depends on a variety of geometrical and optical factors, including the refractive index increment $\mathrm{dn} / \mathrm{dc}$. We assume here that the scattering from free surfactant is negligible.

The expression for the scattering intensity simplifies for small wavevectors and concentrations. When $q R<<1$ the intramicelle scattering interference is negligible and $f(q R<<1) \cong 1$. This condition is easily satisfied in our measurement, where $R \cong 2.5 \mathrm{~nm}$ and $\mathrm{q} \cong 1.05 \times 10^{-2} \mathrm{~nm}^{-1}$. Likewise, when the concentration $\mathrm{c}$ of micelles is small the intermicelle interference is negligible and $\mathrm{S}(\mathrm{q})=1$. Under these conditions $\Delta \mathrm{I}(\mathrm{q})=\mathrm{bc}^{\prime} \mathrm{M}$, and the concentration of micelles should simply be the concentration of surfactant, $c$, minus the concentration of free surfactant, which should be the critical micelle concentration, $\mathrm{cmc}$, at least for concentration close to the $\mathrm{cmc}$, i.e. $\Delta \mathrm{I}(\mathrm{q})=\mathrm{b}(\mathrm{c}-\mathrm{cmc}) \mathrm{M}$. For $r=0 \%$ to $70 \%$ we thus studied the dependence of the micelle scattering intensity as a function of concentration and extrapolated these data to zero concentration, Fig. 1, to find the cmc. From this study we found that the $\mathrm{cmc}$ increases significantly with methanol concentration, Fig. 2. The much lower dielectric constant of methanol ( $\varepsilon=33$ vs. 78 for water) reduces the effectiveness of the chemical dipole of the surfactant, so that the exchange energy of placing a free surfactant into a micelle is less negative. The entropy of mixing of free surfactant is larger than the entropy of mixing of micelles, so the balance is shifted toward more free surfactant.

The extrapolation in Fig. 1 shows that the scattered intensity increases linearly with micelle concentration as long as the surfactant concentration is 


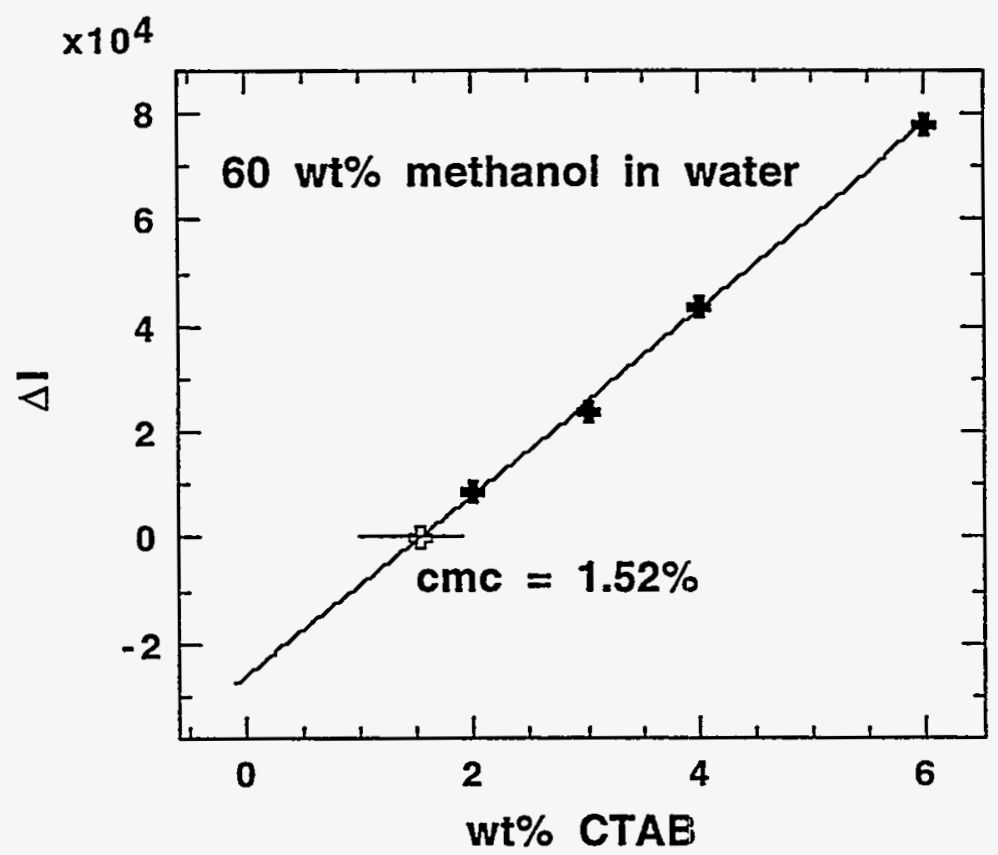

Figure 1. Static light scattering was used to determine the cmc by extrapolating the surfactant contribution to the light scattering to zero scattered intensity.

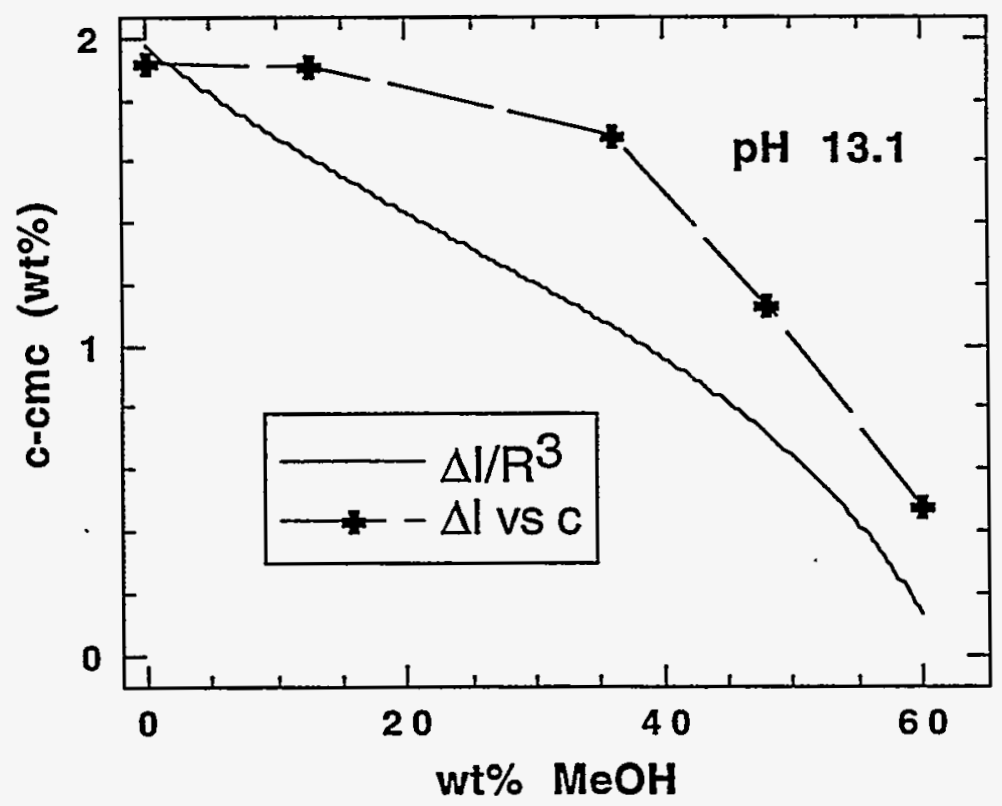

Figure 2. The amount of surfactant in the form of micelles for the mixed solvent system at basic pH was determined by two methods: determining the cmos in Fig. 1 and subtracting this from the surfactant concentration ( $2 \mathrm{wt} \%$ ); and dividing the measured scattering intensity of the 2 $w t \%$ solution by the cube of the hydrodynamic radius. The latter is the continuous line, obtained from the fits to the data in Fig. 4. Although some discrepancies are observed in these two methods, the decrease in micellization with increasing methanol concentration is clear enough. 
less that a few percent, implying that the intermicelle interference, which increases as the micelle concentration squared, is negligible. Thus although at $2 \mathrm{wt} \% \mathrm{C}_{16} \mathrm{TMABr}$ we are not in the strict limit of zero concentration, to a good approximation the relation $\Delta \mathrm{I}(\mathrm{q})=\mathrm{b}(\mathrm{c}-\mathrm{cmc}) \mathrm{M}$ holds. The $\mathrm{cmc}$ can now be determined from the relation $\mathrm{c}-\mathrm{cmc}=\Delta \mathrm{I}(\mathrm{q}) / \mathrm{bM}$. Of course, the micelle mass will in general depend on the solvent composition. We determined the micelle mass by measuring the hydrodynamic radius $R_{h}$ of the micelle from the decay rate of the intensity autocorrelation function, using the StokesEinstein relation for the diffusion coefficient. The dependence of the micelle diffusion coefficient and the solvent viscosity on solvent composition is shown in Fig. 3. The solvent composition dependence of the micelle hydrodynamic radius and solution scattering intensity is shown in Fig. 4. The micelle mass was taken to be $\mathrm{M}=\mathrm{aR}_{\mathrm{h}}{ }^{3}$, so we finally have $\mathrm{c}-\mathrm{cmc}=$ $\Delta \mathrm{I}(\mathrm{q}) /\left(\mathrm{baR}_{\mathrm{h}}{ }^{3}\right)$. [Note that the refractive index of methanol (1.326) is extremely close to water (1.332) so that the variation in optical contrast is negligible.]

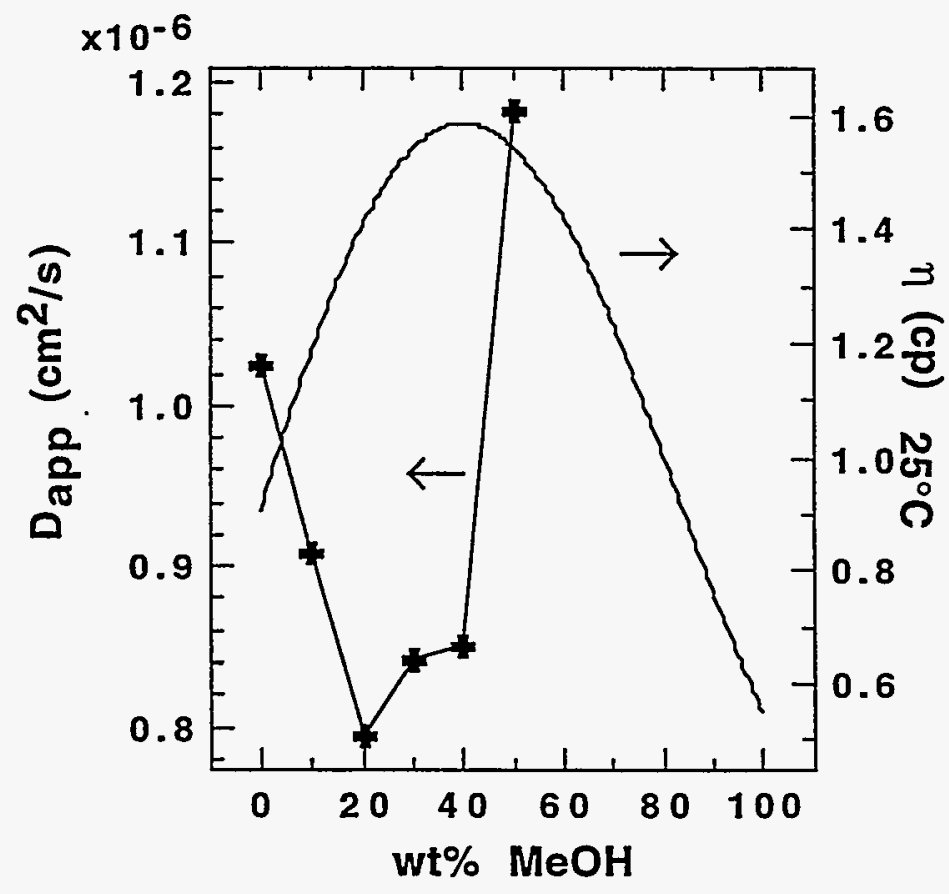

Figure 3. The apparent diffusion coefficient of micelles in a $2 \mathrm{wt} \%$ CTAB solution at $\mathrm{pH} 13.2$ is plotted against the methanol concentration, as is the solvent viscosity. From these data the hydrodynamic radius of the micelles can be determined. 


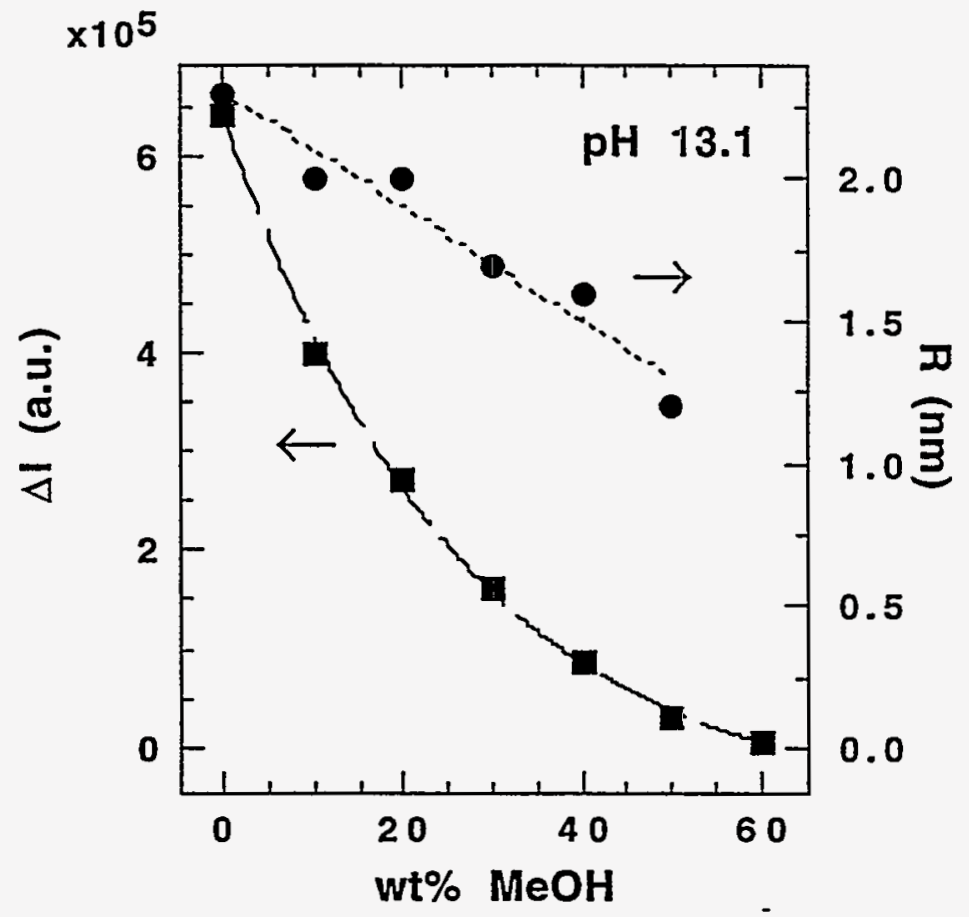

Figure 4. The hydrodynamic radius of the micelles is found to decrease with increasing methanol concentration, and the scattering intensity decreases much more rapidly, which indicates that at $60 \mathrm{wt} \%$ methanol, micelles almost cease to form.

Concentration units can be obtained by noting that our measured $\mathrm{cmc}$ of $\mathrm{C}_{16} \mathrm{TMABr}$ in pure water at $\mathrm{pH}=13.2$ is $0.076 \%$. Equating $\Delta \mathrm{I}(\mathrm{q}) /\left(\mathrm{baR}_{\mathrm{h}}{ }^{3}\right)$ to $c-c m c=(2-0.076)$ gives us the product $a b$. We have plotted the $c-c m c$ versus $w t \% \mathrm{MeOH}$ in Fig. 2, and these data are in reasonable agreement with the numbers computed by subtracting the measured $\mathrm{cmc}$ from the surfactant concentration. The general picture is clear enough: the micelle concentration decreases rapidly with methanol concentration, until the $\mathrm{cmc}$ is approximately equal to the surfactant concentration (2\%) at $60 \mathrm{wt} \%$ methanol. At this point there are essentially no micelles, and it is reasonable to question whether the surfactant can still act as a template.

D.3 Effect of Ionic Strength on Micelle Formation - An additional aspect of these measurements that we found interesting is the increase of the micelle 
scattering intensity with the base concentration, Fig. 5, an increase not associated with a significant change in the cmc. This 8-fold increase in

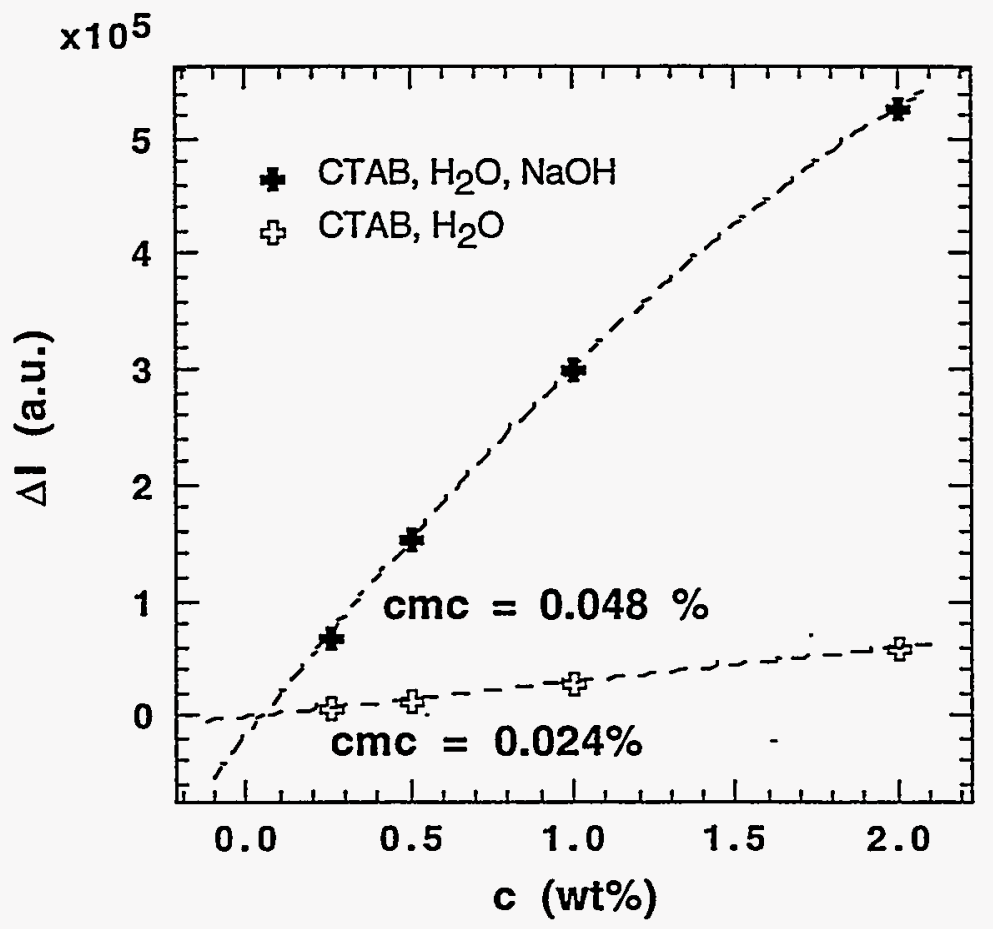

Figure 5. Sodium hydroxide is found to greatly increase the scatting intensity of a CTAB solution, and yet not significantly alter the cmc.

scattering intensity could be a $\mathrm{pH}$ effect or may be due to the increased ionic strength of the basic solution. We found that adding $\mathrm{NaCl}$ to the solution caused a similar increase in scattering intensity, Fig 6, so we believe that an increase in the micelle size may occur due to charge screening: The additional counterions aid in screening the charge interaction between the cationic headgroups, thus reducing the electrostatic contribution to the micelle energy and allowing larger micelles to form. Such effects have been observed in similar systems. [24] To test this directly we used DLS to determine the dependence of the diffusion coefficient and hydrodynamic radius on salt concentration, Fig 7 . These data show that the hydrodynamic radius increases from $0.4 \mathrm{~nm}$ at [salt] $=0 \mathrm{M}$, to $2.5 \mathrm{~nm}$ at [salt] $=0.215 \mathrm{M}$. The radius of a spherical micelle should be about $2.0 \mathrm{~nm}$, so the very small 
aggregates we observe may only be poorly organized clusters of several molecules. In any case, the micelle size and mass is dependent upon the ionic strength of the solution.

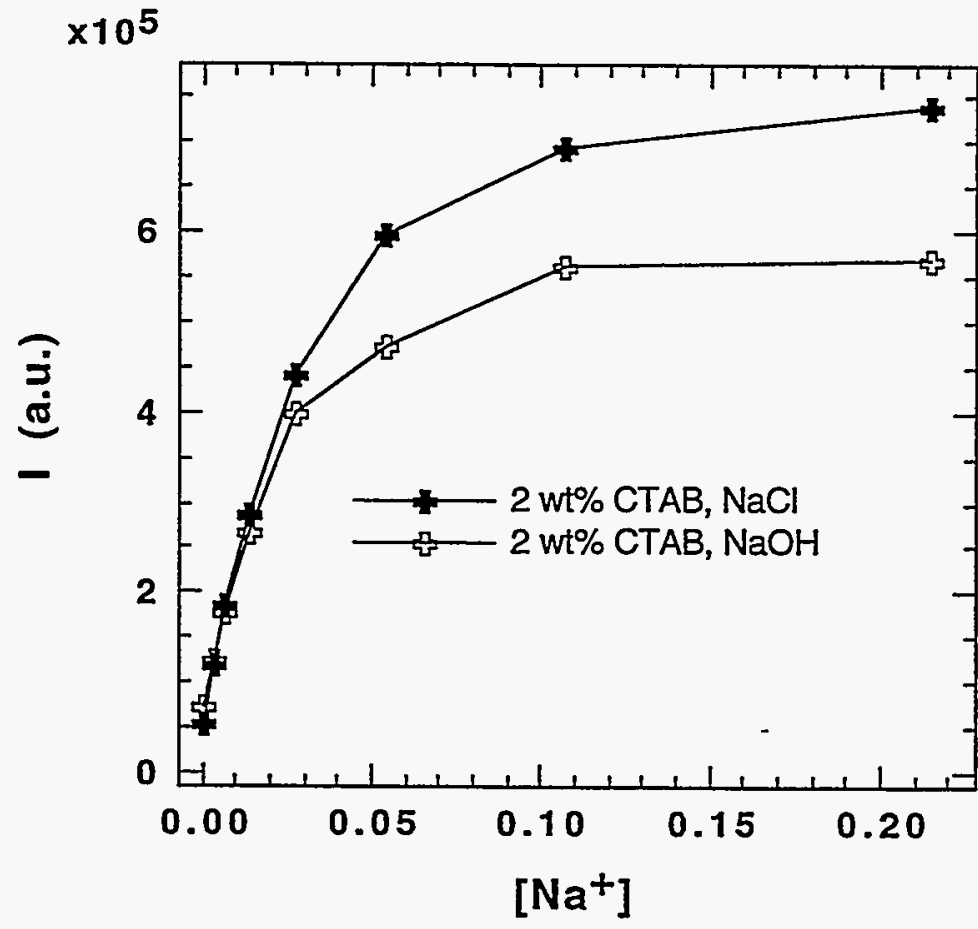

Figure 6. The increase in the scattering intensity we observe with $\mathrm{NaOH}$ can be achieved by adding $\mathrm{NaCl}$, indicating that the effect is not one of $\mathrm{pH}$, but is due to the increased ionic strength of the solution.

D.4 Micellization and Formation Mechanism - It is clear that silica assists the organization of micelles into liquid-crystalline PMS domains. Two key questions are whether the degree of micellization affects the order parameter of the products, and, more generally, whether micelles even need to be present before the silica source is added. The water:methanol system provides an ideal system to answer these questions as the degree of micellization can be continuously varied. Also surfactant solutions with concentrations below $\mathrm{cmc}$ can be made without having to go to extremely dilute systems. 


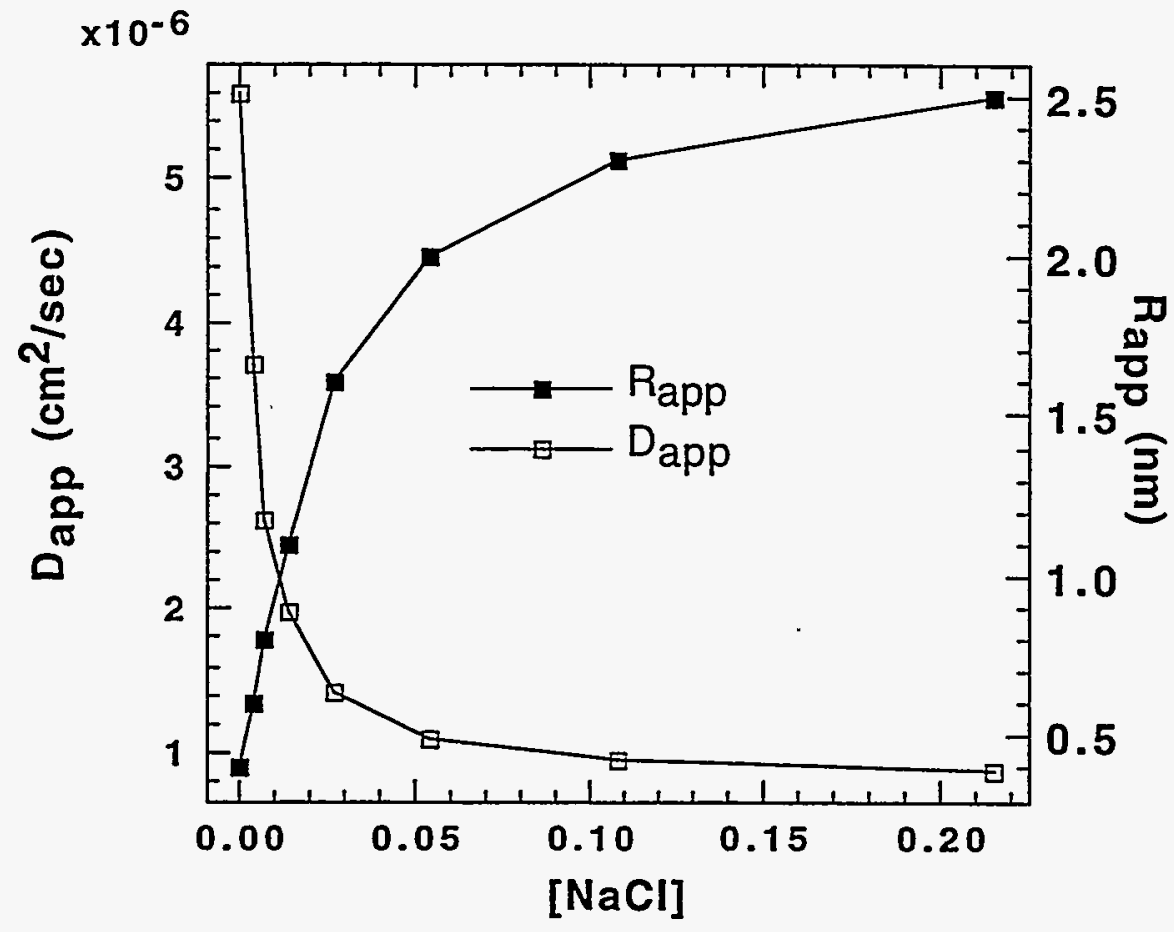

Figure 7. Dynamic light scattering measurements on $2 w t \%$ CTAंB at neutral pH show that with added salt the diffusion coefficient plummets and the hydrodynamic radius increases. The larger micelle size indicates the formation of full micelles at high salt concentration, which causes the increased scattering of light.

To follow the effect of methanol on long-range order we determine the coherent scattering domain (CSD) size from diffraction data, Fig. 8, by applying the Scherrer equation:

$$
\Delta(2 \theta)_{\text {sample }}-\Delta(2 \theta)_{\text {inst }}=0.91 / L \cos \theta_{\text {sampl }}
$$

where $\Delta(2 \theta)_{\text {sample }}$ is the FWHM of the Bragg diffraction peak in radians, $\Delta(2 q)_{\text {inst }}$ is the instrumental broadening of the peak, [25] $\lambda$ is the incident radiation wavelength (1.54056 $\AA$ ), $\theta_{\text {sample }}$ is the position of the peak, and $L$ is the coherent scattering domain (CSD) size in Angstroms. As Fig. 9 shows, the CSD increases at low $r$ to a maximum at $\sim 20$ to $25 \%$, and then decreases until $c »$ $c m c$, at which point there is a relatively sharp decrease. In all cases the average particle sizes of the products are larger than the CSD size, so the 


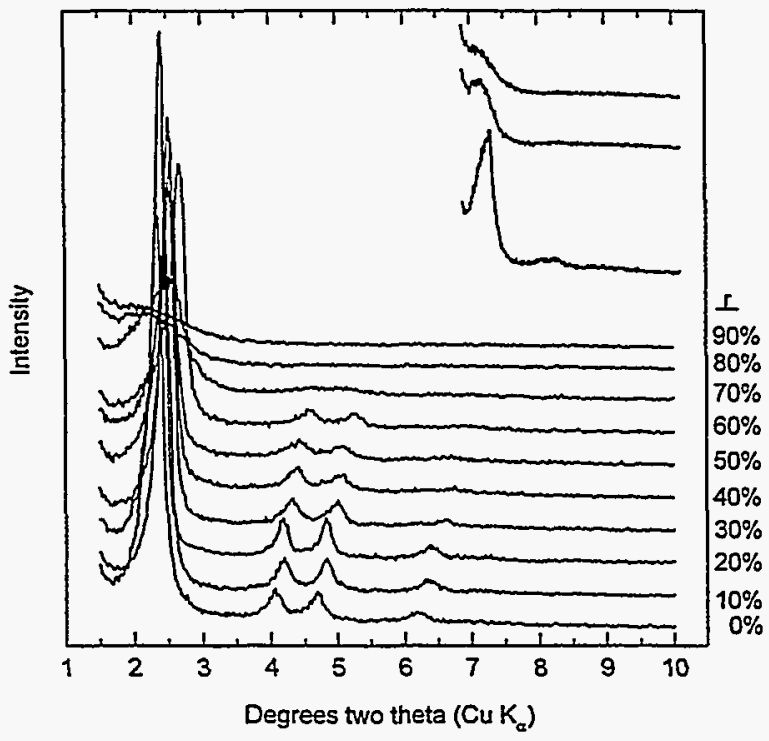

Figure 8. X-ray diffraction data show the decrease in number of peaks as $r$ increases, which indicates a reduction in long-range order. Note the shift of the peaks to smaller $d$ values as $r$ increases, which indicates a reduction in the unit cell constant. -The inset shows from top to bottom $r=10 \%, 20 \%$ and $30 \%$. These samples were washed with methanol to avoid the possibility of converting unreacted reagents to PMS. Adding water to the filtrate produced no solids.

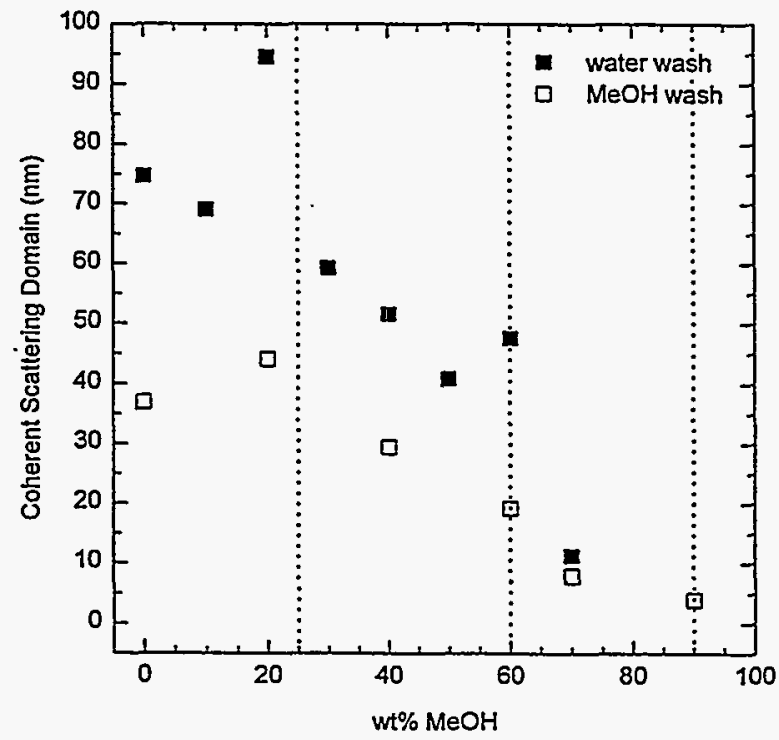

Figure 9. The long-range order of silica/surfactant mesophases is a maximum at $-20-25 \mathrm{wt} \%$ methanol for samples washed with water (circles) and for samples washed with methanol (triangles). There is a precipitous drop in $C S D$ at $c-c m c=0$. Our best sample made at $10^{\circ} \mathrm{C}$ in 75:25 water methanol has a CSD of $140 \mathrm{~nm}$. 
broadening of the Bragg peaks is a measure of the long-range order within a particle, rather than a measure of particle size.

As shown in Fig. 2, the greatest extent of micellization in the water:methanol system occurs for no added methanol. Therefore, the materials with the longest range order may be expected to occur with no added methanol. The maximum CSD actually occurs at $r$ »25\%, which is the concentration at which $t_{\text {solubility }}$ first exceeds $t_{\text {gel }}$. [26] Thus we have shown that to achieve optimal long-range it is important for $t_{\text {gel }}>t_{\text {solubility }}$ that is, that the precursor solution is homogeneous prior to phase separation into product and liquid.

In the region $30 \%<r<70 \%$ the CSD slowly decreases in parallel with the steady decrease $c-c m c$, which shows that the degree of micelliztion in the precursor solution affects the degree of order in the product. At $r \gg 70 \%$ the CSD decreases abruptly as $c$ exceeds $c m c$. This dramatically illustrates the effect of surfactant preorganization on product order. For $70<r<90$ despite the very small CSD sizes as evidenced by one broad peak in the XRD pattern, TEM micrographs confirm a honeycomb-like porous structure. Gas adsorption measurements on a calcined material made with $r=30 \%$ confirm that the template can be removed from the mesophase yield a periodic mesoporous product with surface area of $317 \mathrm{~m}^{2} / \mathrm{g}$ and porosity of $0.20 \mathrm{cc} / \mathrm{g}$.

At $r>90 \%$, translucent chemical gels form. Gelation times are typically several order of magnitude longer than for mesophases. The water:silicon ratio $(h)$ is less than 10 in this regime. The value of $h$ is not excessively low mesophases are typically from solutions with $h \sim 17$ to $25,[6,12]$ and microporous silicate films are made commonly with $h$ less than $5[27]-$ but the presence of methanol shifts the hydrolysis and the condensation equilibria toward non-hydrolyzed precursors and non-condensed silicate species, which retards the overall reaction rate. In this low dielectric constant environment it is clear that the surfactant has ceased to act as an effective chemical dipole and that the impetus for cooperative assembly into an ordered product is gone. 


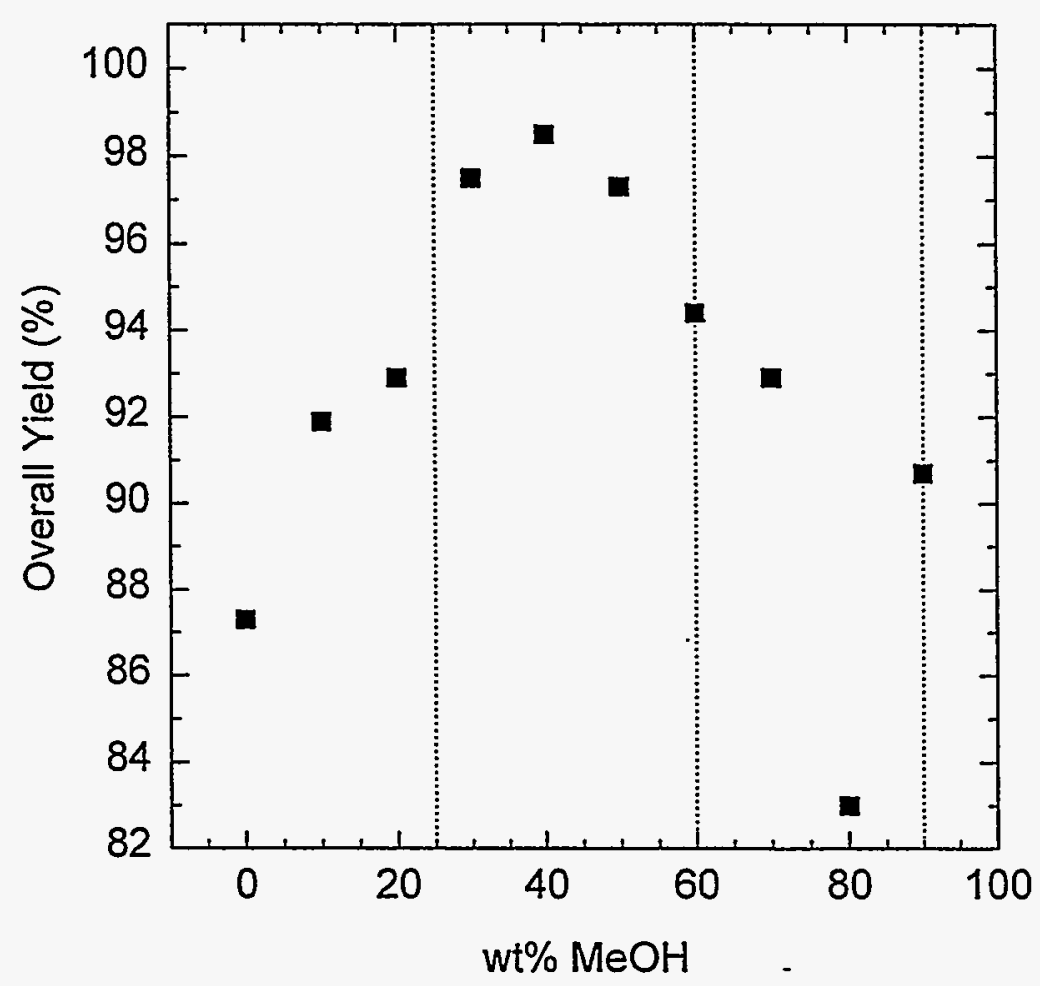

Figure 10. Yield of solid products from samples made from reaction of $4.2 \mathrm{mmol}$ TMOS with $10 \mathrm{~g}$ of an alkaline $2 \mathrm{wt} \% \mathrm{C}_{16} \mathrm{TMABr}$ solution. Overall yield $=100 *$ (1- (wt. filtrate residue - calc. wt. $\mathrm{NaBr}$ )/ theoretical yield of $\mathrm{SiO}_{2.13} \cdot 0.13 \mathrm{C}_{16} \mathrm{TMA}^{+}$).

The link between micellization and long-range order of the product is now clear. The materials that have the largest CSD sizes are made from solutions that are homogeneous and that have the maximum preorganization of surfactant [28] - in our case, solutions that have $r=\sim 20$ to $25 \%$. Micellization of the surfactant prior to the addition of the silica source does substantially improve the long-range order of the hexagonally arrayed pores; however, the prearrangement of surfactant molecules into micelles is not necessary to the formation of periodic ordered mesophases.

D.5 Effect of Methanol on Product Yield -- We established that methanol dissolves all of the TMOS prior to gel formation for $r>\sim 25 \%$, and thus we expect materials made from these solutions to exhibit higher yield that those made with $r<25 \%$. As shown in Fig. 10 this is the case. In fact as long as $t_{g e l}>$ $t_{\text {solubility }}$ and there are micelles in solution initially, the yield is nearly 


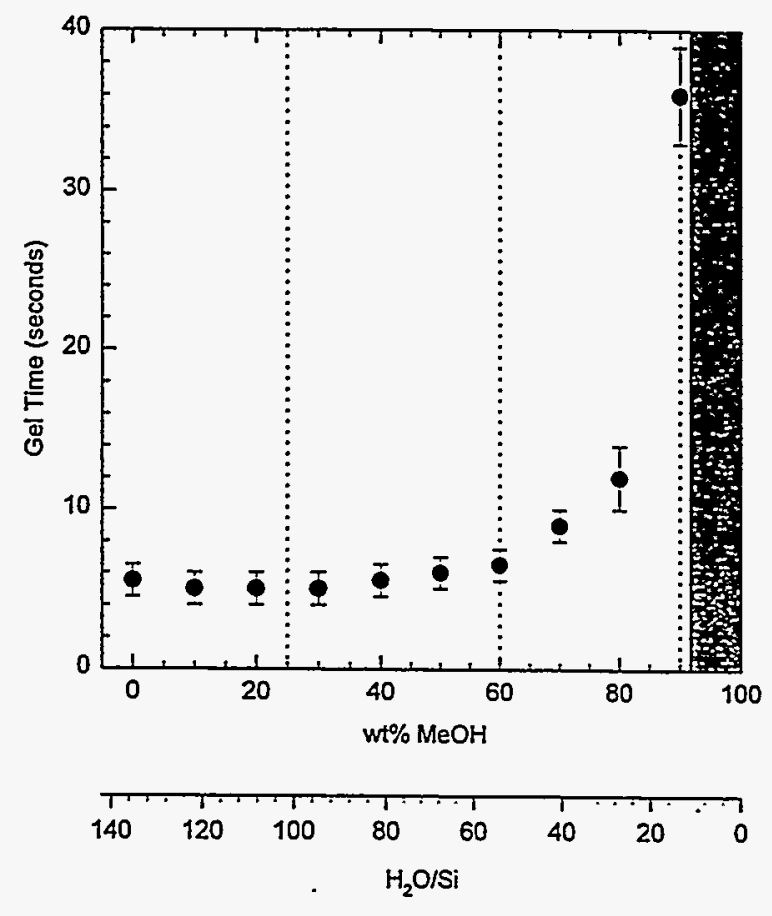

Figure 11. The gel time is nearly constant for $c-c m c>0$, but increases rapidly for $c-c m c<0$. The increase is likely partly attributable to the diminishing concentration of water, but primarily attributable to the failure of the surfactant to act as a template. At $\mathrm{H}_{2} \mathrm{O} / \mathrm{Si}<10$ chemical gels of amorphous silica form as opposed to periodic mesophases. Samples were made from reaction of $4.2 \mathrm{mmol}$ TMOS with $10 \mathrm{~g}$ of an alkaline $2 \mathrm{wt} \% \mathrm{C}_{16} \mathrm{TMABr}$ solution.

quantitative. For $r<25 \%$ some of the silica is presumably in the form of amorphous byproducts and thus not all of the surfactant is used in the reaction and is thus collected in the filtrate. For $r>60 \%$ it appears that not only is the long range order of the product less than optimal, but the complete transformation to periodic product is frustrated and some of the free surfactant and small silica species are left unreacted.

D.6 Effect of TMOS and Methanol on Kinetics - The use of the rapidly hydrolyzable alkoxide TMOS in basic solution leads to the rapid evolution of charged silicate species and the formation of a particulate gel within a few seconds Fig. 11. Amazingly, as shown in Fig. 12, the wet gels have periodic order at the earliest time we can make measurements, $\sim 1 \mathrm{~min}$. 


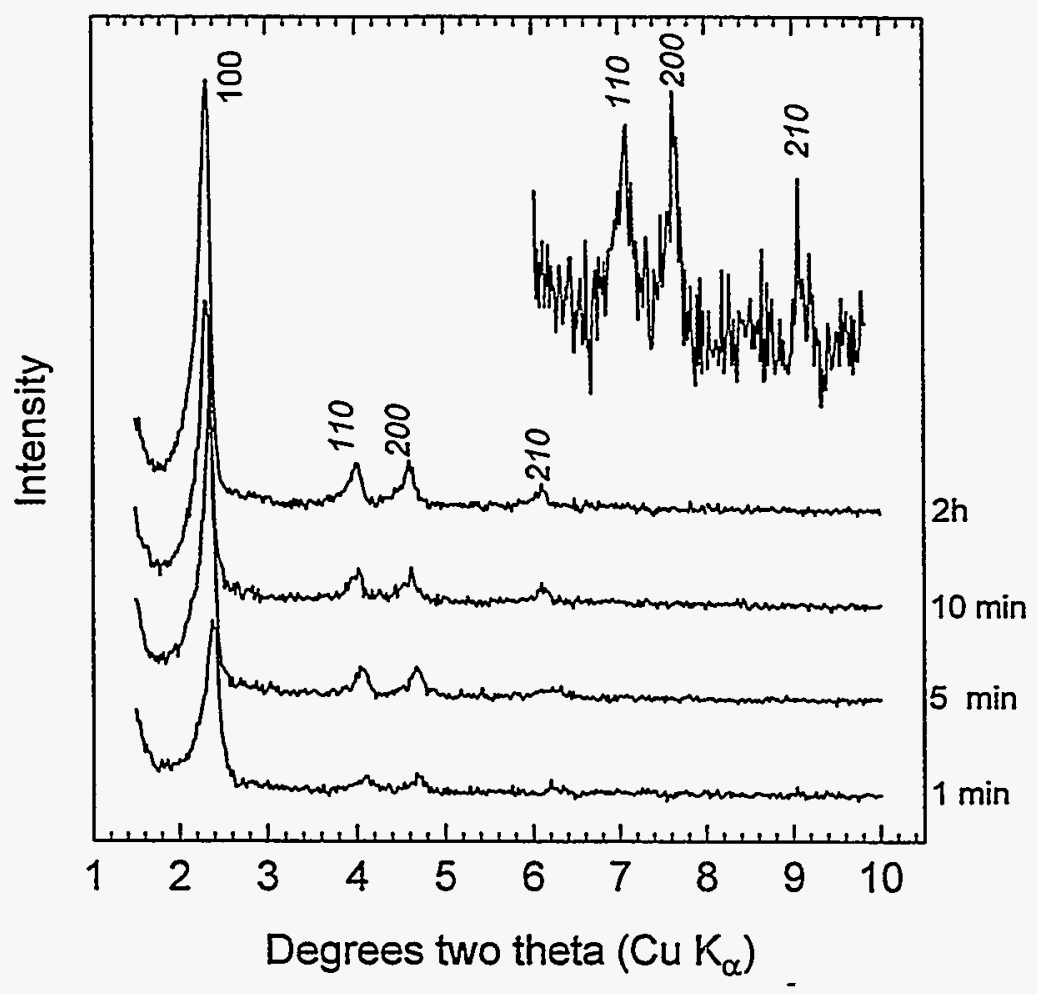

Figure 12. In situ $X$-ray diffraction of mesophases made from reaction of $4.2 \mathrm{mmol}$ TMOS with $10 \mathrm{~g}$ of an alkaline 75:25 water:methanol $2 \mathrm{wt} \% \mathrm{C}_{16} \mathrm{TMABr}$ solution. Samples were isolated by filtration. Recovered wet gels were immediately transferred to a holder. The Miller indicies for each Bragg reflection are shown. The inset shows the sample analyzed at 1 minute.

The formation time with TMOS is 1 to 2 orders of magnitude faster than for TEOS and 3 to 4 several hours for samples made from nonmolecular silica sources. It is well known that the hydrolysis time for alkoxysilanes increases as the size of the alkoxide ligand increases, which accounts for the difference between TEOS and TMOS. It is also well known that, even at high temperatures and a $\mathrm{pH}$ of $\sim 12$, the dissolution of solid of colloidal silica is relatively slow, which accounts for the long times used to form mesophases derived from these silica sources. These temporal differences imply that the rate limiting step in mesophase formation is the evolution of the small, charged silicate oligomers that compose the inorganic framework.

D.7 Effect of TMOS and Methanol on Microstructure -- The tertiary structure of the TMOS derived periodic products is distinctly different from materials 


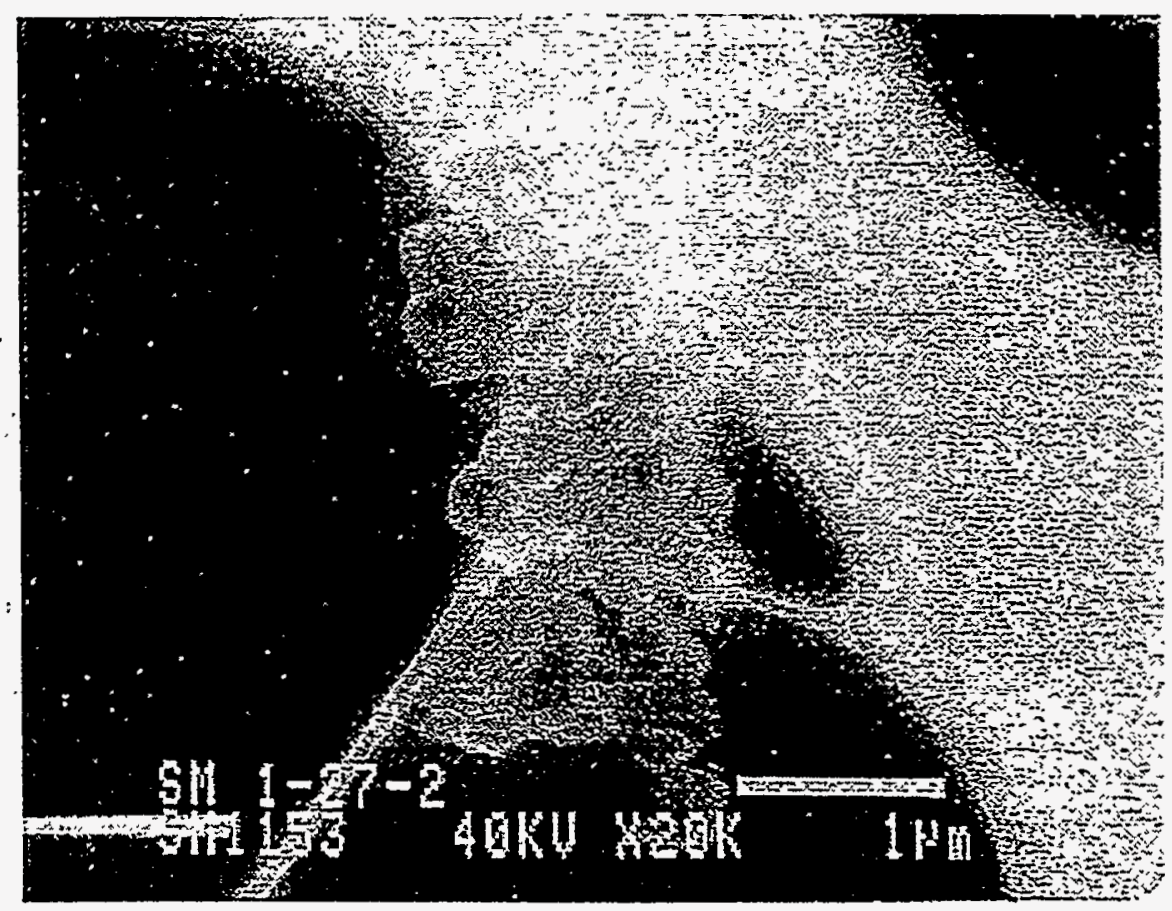

Figure 13. Scanning electron micrographs of samples prepared from reaction of $4.2 \mathrm{mmol}$ TMOS with $10 \mathrm{~g}$ of an alkaline 75:25 water:methanol $2 \mathrm{wt} \% \mathrm{C}_{16} \mathrm{TMABr}$ solution. The micrograph clearly shows the aggregated submicron crystals. Particle size ranges from $\sim 150$ to $\sim 500 \mathrm{~nm}$.

made from non-molecular sources or from $\mathrm{Si}(\mathrm{OR})_{4}$ where $\mathrm{R}=\mathrm{C}_{2} \mathrm{H}_{5}, \mathrm{C}_{3} \mathrm{H}_{7}$ $\mathrm{C}_{4} \mathrm{H}_{9}$. TMOS gives weak particulate gels rather than colloidal precipitates. The aggregated particles that compose the gel are submicron and spherical or ellipsoidal, Fig. 13. They typically range in size from 0.15 to $0.5 \mathrm{~mm}$. We have examined the structure and absorptive properties of the gel monoliths, and details are reported elsewhere. [29] After breaking apart the gel and washing with water or methanol, aggregates of with dimensions greater than $1 \mathrm{~mm}$ are present, but the contact between individual particles is minimal. The microstructure of the broken gels provides ready access to the internal surface area of each particle and facilitates transport into and out of the powder. High resolution TEM confirms the regular honeycomb arrangement of the pores within each particle, Fig. 14. 


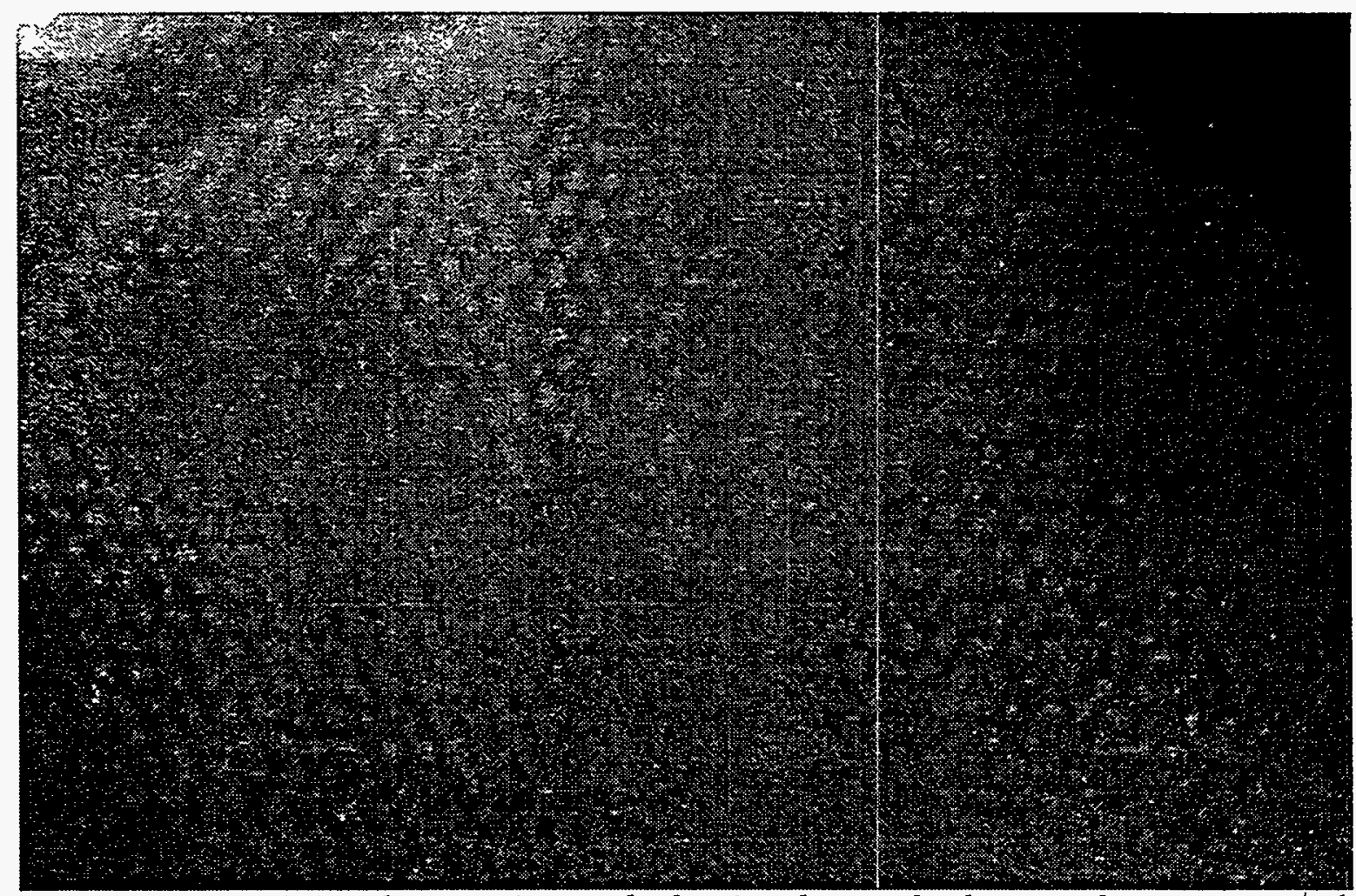

Figure 14. Transmission electron micrograph showing the regular honeycomb arrangement of pores in the uncalcined nanocomposite. The sample was made from reaction of $4.2 \mathrm{mmol}$ TMOS with $10 \mathrm{~g}$ of an alkaline 75:25 water:methanol $2 \mathrm{wt} \% \mathrm{C}_{16} \mathrm{TMABr}$ solution.

\section{D.8 Effect of TMOS and Methanol on Framework Condensation - The ${ }^{29} \mathrm{Si}$} MASNMR measurements on $r=25 \%$ gels isolated 1 minute after formation show that there is a very high fraction of uncondensed silicon groups present, Fig. 15. The $Q^{3} / Q^{4}$ ratio is $\sim 2.1$ as compared to $\sim 1.2$ for a material made from TEOS in water (isolated after $10 \mathrm{~min}$ ). Over time the framework with TMOS continues to condense to form siloxane bonds even when aged in the original 75:25 mother liquor. After one week the $Q^{3} / Q^{4}$ ratio in an aged sample is $\sim 1.2$. After the materials are calcined, the $Q^{3} / Q^{4}$ ratio is $\sim 0.40$, which is nearly identical to that for samples made from TEOS. This is important, as it means that the framework from the TMOS-derived samples is ultimately as well condensed as materials made from other techniques and should exhibit similar chemical and hydrothermal stability. 


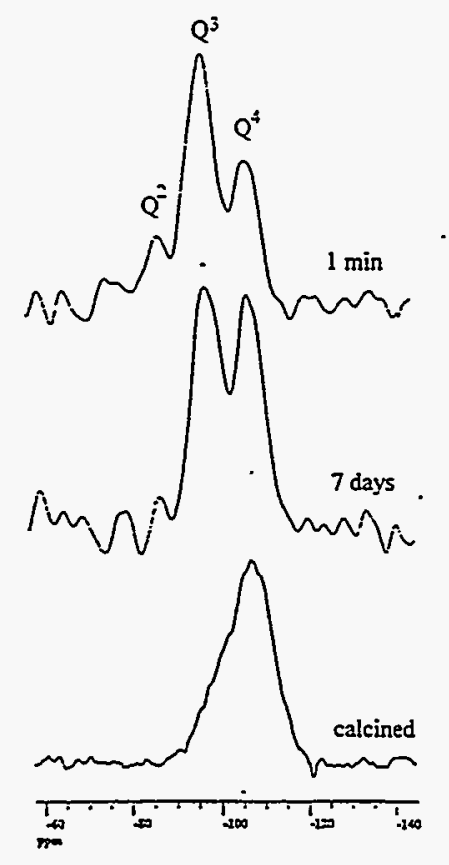

Figure 15. ${ }^{29} \mathrm{Si}$ MAS NMR show the $Q^{3} / Q^{4}$ evolution as a function of aging time and processing conditions. The top spectrum is for a sample isolated after 1 minute with data collected over $\sim 18 \mathrm{~h}\left(Q^{3} / Q^{4}=2.1\right)$, the middle is for a sample aged in its mother liquor for $7 \mathrm{~d}\left(Q^{3} / Q^{4}=1.2\right)$, and the third is for a sample calcined in $\mathrm{N}_{2}$ and $\mathrm{O}_{2}$ at $550^{\circ} \mathrm{C}$ with a heating rate of $10^{\circ} \mathrm{C} / \mathrm{min}$ $\left(Q^{3} / Q^{4} \gg 0.40\right)$. All samples were prepared the preferred synthetic method. Peak positions: 1 minute $-Q^{2}=-88.2 \mathrm{ppm}, \mathrm{Q}^{3}=-98.8 \mathrm{ppm}, \mathrm{Q}^{4}=-107.8 \mathrm{ppm} ; 7 \mathrm{~d}-\mathrm{Q}^{2}=-87.6 \mathrm{ppm}, \mathrm{Q}^{3}=-98.5 \mathrm{ppm}, \mathrm{Q}^{4}$ $=-107.8 \mathrm{ppm}$; calcined $-\mathrm{Q}^{2}=-88.8 \mathrm{ppm}, \mathrm{Q}^{3}=-98.0 \mathrm{ppm}, \mathrm{Q}^{4}=-106.7 \mathrm{ppm}$.

\section{D.9 Effect of Methanol on Micelle Radii and Pore Diameter -- Continuous} control of pore diameter is afforded by changing the water:methanol ratio. From dynamic light scattering experiments we observe that addition of methanol to the micellar solution decreases the hydrodynamic radius of micelles, Fig. 16. This decrease in micelle radius is paralleled by a decrease in unit cell constant in resulting silica-surfactant composites. The decrease in cell constants is indicative of a decrease in pore diameter of the calcined porous products as confirmed by gas adsorption studies. The value of $r$ can be changed from $0 \%$ to $\sim 70 \%$ to provide a continuous $\sim 5 \AA$ span of pore sizes.

D.10 Controlling Porosity - The porosity of the 75:25 water:methanol derived periodic silica is dictated by the method used to remove the template. 


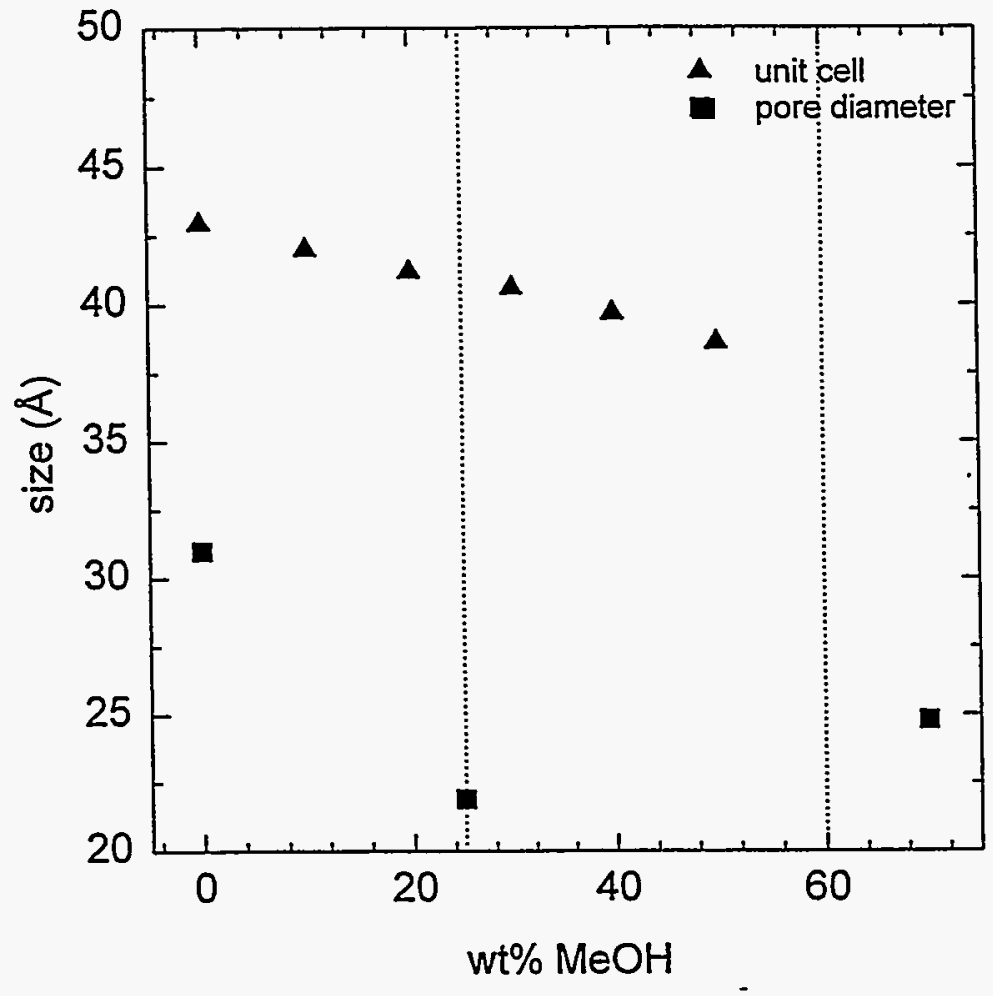

Figure 16. The unit cell parameters and pore radius decrease as $r$ increases until $c-c m c=0$. The cell radius and pore diameter are larger when no micelles are present than when the concentration is low.

Calcination leads to greater surface area and pore volumes than liquid extraction of the solvent. For example, a 75:25 water:methanol sample extracted with acidic ethanol $(\mathrm{pH}=2.5)$ at room temperature for $93 \mathrm{~h}$ had a surface area of $210 \mathrm{~m}^{2} / \mathrm{g}$ and a pore volume of $0.14 \mathrm{cc} / \mathrm{g}$, Fig. 17. From TGA studies on the porous product, the removal of organic material seems nearly complete; however, extraction in ethanol may very well lead to significant breaking of Si-O-Si bonds to form Si-O-Et linkages. This weakens the framework could lead to its partial collapse. The framework collapse is not unexpected given the high fraction of $Q^{3}$ species present in the as-made material.

In contrast, calcined materials made with 75:25 $\mathrm{H}_{2} \mathrm{O}: \mathrm{MeOH}$ have surface areas as high as $970 \mathrm{~m}^{2} / \mathrm{g}$ and pore volumes up to $0.66 \mathrm{cc} / \mathrm{g}$, Fig. 18 . The high temperatures and absence of solvents drive the condensation of 


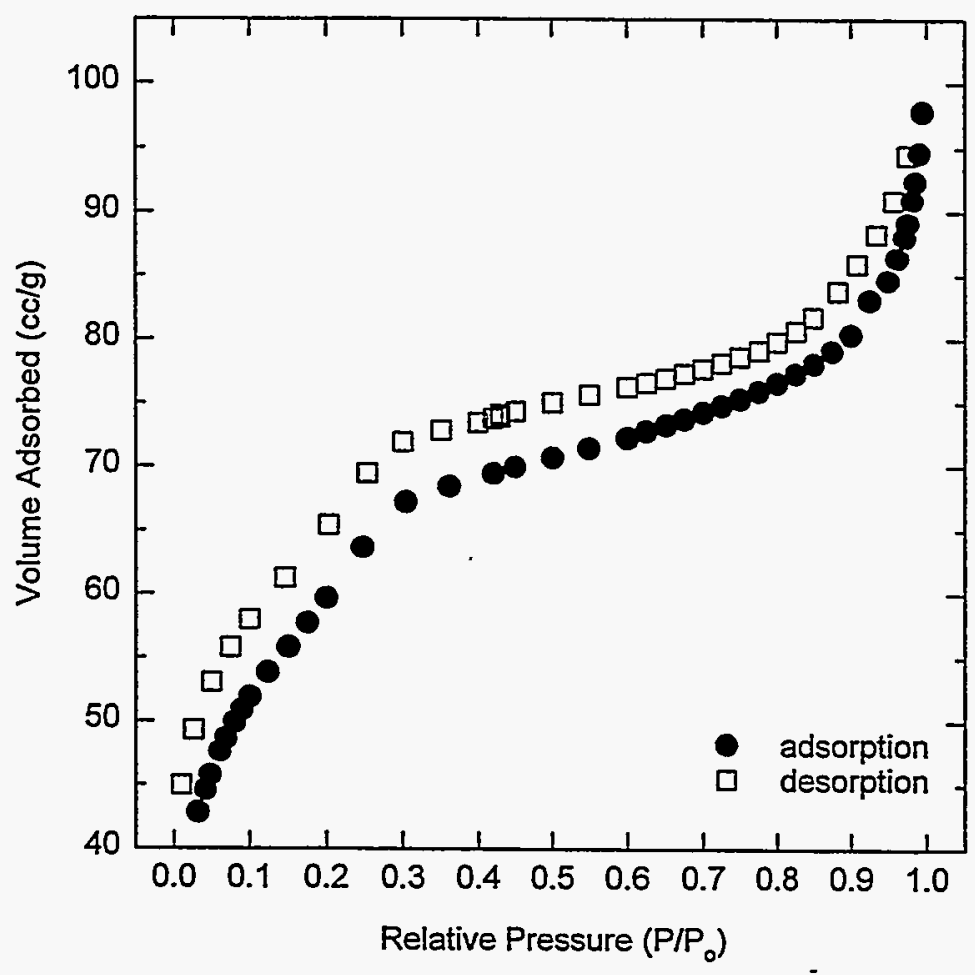

Figure 17. Nitrogen sorption isotherm at $77 \mathrm{~K}$ of a solvent exchanged sample. The surface area is $213 \mathrm{~m}^{2} / \mathrm{g}$ and the pore volume is $0.14 \mathrm{cc} / \mathrm{g}$. The average pore radius by $2 \mathrm{~V} / \mathrm{A}$ from BET analysis is $\sim 13.4 \AA$, indicating a mesoporous material. Equilibrium was not achieved at each point, thus the hysteresis. The sample was prepared by reaction of $4.2 \mathrm{mmol}$ TMOS with $10 \mathrm{~g}$ of an alkaline $2 \mathrm{wt} \% \mathrm{C}_{16}$ TMACl 75:25 water:methanol solution. Template was extracted in acidic anhydrous ethanol (1.3 M HCl) for $93 \mathrm{~h}$ at room temperature. The solid to solvent ratio was $1 \mathrm{~g} / 200 \mathrm{~mL}$. The sample lost $40.7 \%$ of its original weight during extraction. Thermogravimetric analysis showed a 9.3\% weight loss for the extracted sample, which corresponds to the amount of water adsorbed when the porous sample is left in air, i.e. almost no organic was present.

silicate species to form a stable bonded silica framework. Surface area porosity is thus maintained.

The final pore diameter of PMS depends calcination conditions used to remove the organic phase. For calcined samples, the shrinkage is in large part determined by the gas used to during the heat treatment, Fig. 19. Shinkages typically range from $\sim 10$ to $\sim 20 \%$ for samples aged $5 \mathrm{~min}$ to $50 \mathrm{~h}$ prior to calcination. Shinkages are on the high end of the range when the samples are heated only in $\mathrm{O}_{2}$. The shrinkage can be lessened if the sample is first calcined in $\mathrm{N}_{2}$. Calcination in $\mathrm{N}_{2}$ to $550^{\circ} \mathrm{C}$ removes greater than $\sim 95 \%$ of the organic material. The organic matter decomposes at higher temperature in $\mathrm{N}_{2}$ 


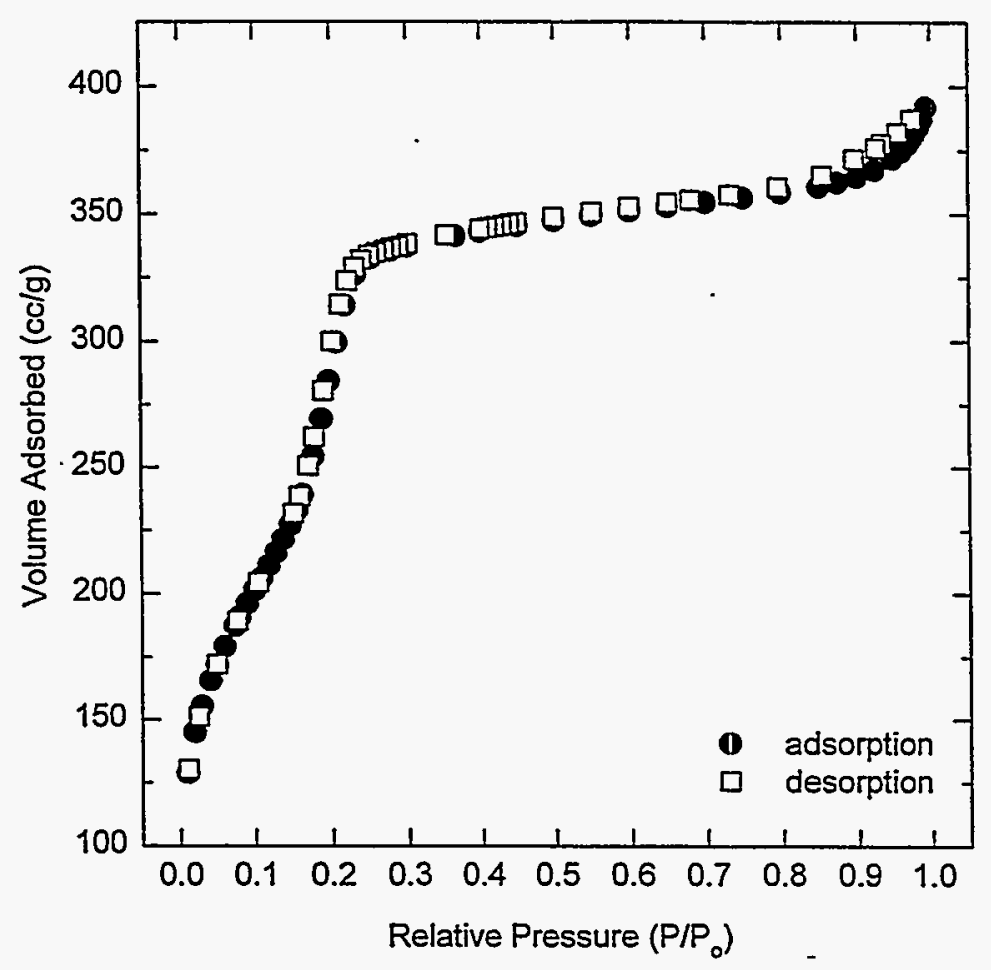

Figure 18. Nitrogen sorption isotherm at $77 \mathrm{~K}$ on a calcined sample. The surface area is $902 \mathrm{~m}^{2} / \mathrm{g}$ and the pore volume is $0.61 \mathrm{cc} / \mathrm{g}$. The average pore radius by $2 \mathrm{~V} / \mathrm{A}$ from $\mathrm{BET}$ is $\sim 13.4 \AA$, which indicates a mesoporous material. The sample was prepared by reaction of $4.2 \mathrm{mmol}$ TMOS with $10 \mathrm{~g}$ of an alkaline $2 \mathrm{wt} \% \mathrm{C}_{16} \mathrm{TMACl}$ 75:25 water:methanol solution. Template was removed by calcination in flowing $\mathrm{O}_{2}$. Heating rate was $\sim 4.4^{\circ} / \mathrm{min}$ to $550^{\circ} \mathrm{C}$ followed by a $2 \mathrm{~h}$ isotherm and slow cool to room temperature.

than in $\mathrm{O}_{2}$. Delaying decomposition allows for greater framework bonding prior the very exothermic decomposition of organic groups. The stabilized network shrinks less when the template is removed. The small amount of residual organic matter not decomposed by $\mathrm{N}_{2}$ is removed by subsequent calcination in $\mathrm{O}_{2}$.

Stepwise control of pore diameter has been demonstrated in the $r=$ $25 \%$ series by using $\mathrm{C}_{\mathrm{n}} \mathrm{H}_{2 \mathrm{n}+1} \mathrm{~N}\left(\mathrm{CH}_{3}\right)_{3} \mathrm{Br}$ with $n=10,12,14,16,18$. The cell constant change is given approximately by $2.25 \Delta n \AA$ in this range, which is very similar to what has been found in purely aqueous systems. [1,3] This indicates that the surfactant chains are extended $\sim 89 \%$ of maximum.

The pore size distribution (psd) and pore diameter are influenced by the water:methanol ratio. Samples with $r=0 \%, 25 \%$, and $70 \%$ were studied 


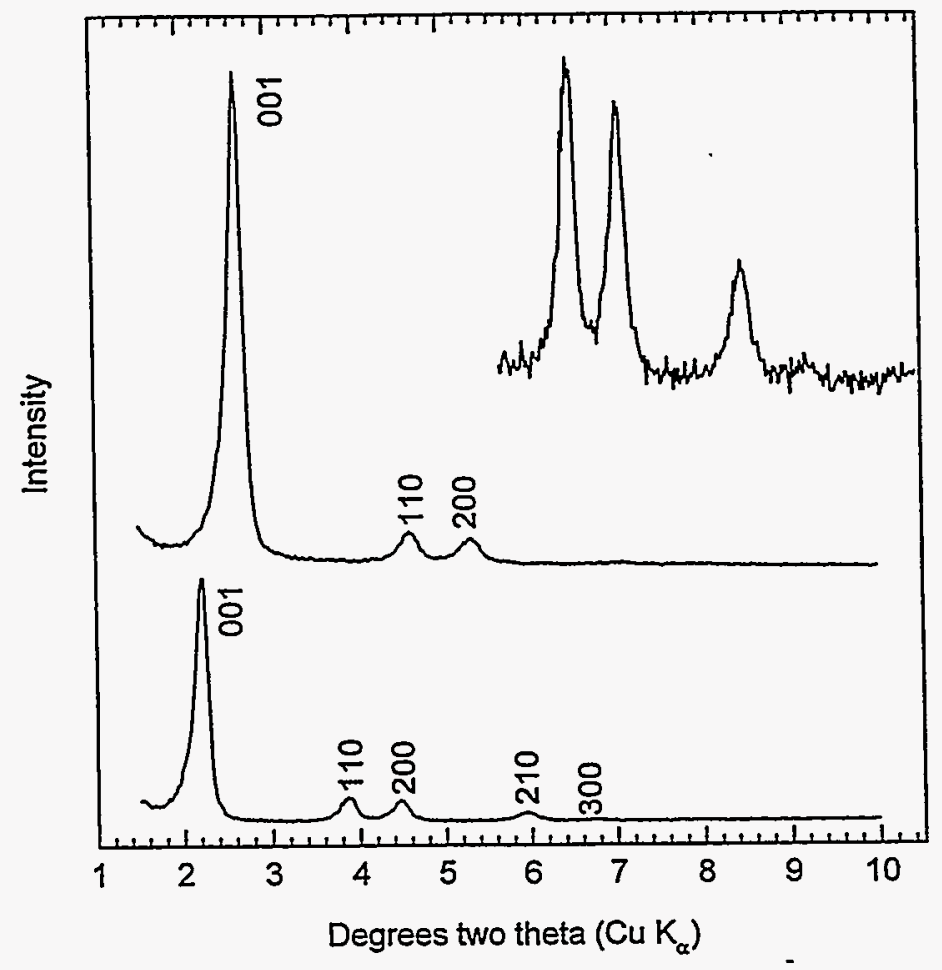

Figure 19. X-ray diffraction of calcined sample described in Fig. 18. The cell parameter shrinks from 45.36 (10) in the as-made samples to 38.44 (5) $\AA$ in the calcined sample. The overall shrinkage is $15.3 \%$. Note that the long-range order of the calcined product is less than for that of the uncalcined as evidenced by the absence of the 210 and 300 Bragg reflections.

in detail. The psd is a minimum, and the surface area is a maximum at $r=$ $25 \%$. The pore diameter decreases from $r=100 \%(\sim 31 \AA)$ to $r=30 \%(\sim 25 \AA)$. The wall thickness varies from $\sim 8 \AA$ to $\sim 14 \AA$ depending on the sample, but there appear to be no general trends with $r$.

\section{E. Conclusions}

We have found that homogenous solutions of molecular precursors that still support micelle formation are necessary to optimize the yield, longrange order, and adsorptive properties of PMS. In particular, the cosolvent methanol enables us to achieve such homogenous, micelle-forming solutionsd. A number of experimental measurements on the mesoporous product demonstrate that the optimal water:methanol ratio is $75: 25$, but satisfactory product is made over a wide methanol range. The cosolvent 
methanol also allows the pore size and distribution to be tailored over a wide continuous range.

Light scattering measurements indicate that the micelle size and the $\mathrm{cmc}$ are strong functions of methanol concentration, $\mathrm{pH}$, and added salt. The formation of micelles is not a sufficient condition to insure the formation of PMSs: at high added salt and neutral $\mathrm{pH}$ micelles do form, but the product does not.

The reactions we have described result in gels that consist of mesoporous particles that are $\sim 250 \mathrm{~nm}$ in size. These particulate gels are formed by the aggregation of particles and the gelation of aggregates. The morphology and minimal connectivity of particles in these gels is advantageous for gas adsorption applications. The inorganic framework of the gels is initially poorly condensed, but aging and calcination provide a well condensed, stable siloxane network.

\section{Synthesis of Periodic Mesoporous Silica Thin Films}

\section{A. Epitome}

We describe a new synthetic method for the formation of thin films of periodic mesoporous silica. This method yields thin films that are continuous and crack-free, that have the familiar hexagonal structure, and that have lattice parameters of $\sim 40 \AA$. The method, which we call GasCatalyzed Thin Film Synthesis (GCTFS), involves diffusing ammonia into a homogeneous micellar coating solution on a substrate. This method ameliorates problems associated with coating substrates from inhomogeneous solutions and provides films that have a well defined microstructure and surface areas greater than $300 \mathrm{~m}^{2} / \mathrm{g}$. We have studied and optimized GCTFS with light scattering, ${ }^{29} \mathrm{Si}$ NMR, XRD, optical microscopy, SEM, TEM, and isothermal $\mathrm{N}_{2}$ sorption measurements. 


\section{B. Introduction}

Thin films of periodic mesoporous silica have a number of possible applications, which include size-specific coatings for surface acoustic wave (SAW) sensors, [20] catalyst supports, [16,29] and funtionalized optical coatings. There has been some discussion of the use of mesoporous silica as a surface coating in the literature. For example, in the applications patent by Olson, Stucky, and Vartuli, [20] a bulk phase was dispersed into a liquid and dip-coated onto a substrate, which resulted in a noncontinuous, non-uniform coating of colloidal particles that had been homogeneously nucleated in solution. Ogawa [30] reported the formation of lamellar thin films from acid catalyzed solutions of silica at low silica to surfactant ratios. At high silica concentrations the hexagonal phase did not form. Yang et al. [31] reported a very nice study on the growth of hexagonal thin films of mesoporous silica on a mica substrate suspended horizontally in an acidic solution of tetraethoxysilane (TEOS). The pores tended to align parallel to the surface, and the accessible pore volume was not reported. Bontha et al. [32] reported the synthesis of mesoporous films through liquid-liquid interfacial reactions.

The formation of thin films of mesoporous silica is nonetheless vexed by several problems: (1) particles nucleate in solution, precipitate, and sediment onto the substrate, (2) the kinetics of product formation are slow so there is a wide distribution of species in solution at any given time, any of which can be deposited onto the substrate (3) the solutions used to make the films or coatable powders are inhomogeneous, so that byproducts, such as, amorphous silica and excess surfactant, can be incorporated into the films, (4) the microstructure of the films derived from solution, or particles spread onto a substrate, is difficult to control due to large variations in particle size and agglomeration.

To overcome these problems, we devised a method to rapidly form products from homogeneous solutions that have a controlled, predictable microstructure. The method builds on our previous work making 
mesoporous silica gels from the molecular precursor TMOS. [33] The method has the advantage that the solutions can be easily dip-or spin-coated to give films with complete, continuous coverage, very large accessible pore volume, and that contain a minimum of byproducts. We are able to make films of aggregated 20-500 nm periodic mesoporous particles; within each particle exists the familiar hexagonal array of $2-4 \mathrm{~nm}$ diameter channels. The film thickness can be varied from a few hundred nanometers to several microns. The films are stable to calcination at $500{ }^{\circ} \mathrm{C}$ and exhibit surface areas in excess of $300 \mathrm{~m}^{2} / \mathrm{g}$. The films are being evaluated as high surface area, size-selective coatings for surface acoustic wave (SAW) sensors.

\section{Experimental}

C.1 Preparative - A typical synthesis of periodic mesoporous silica involves mixing deionized water, a cosolvent, such as, methanol, ethylene glycol, or formamide, cetyltrimethylammonium bromide or hydrogensulfate (CTAB or CTAHS), $\mathrm{NaOH}$ or $\mathrm{NH}_{3}$ gas, and tetramethoxysilane (TMOS). Two general preparations are solutions catalyzed with $\mathrm{NaOH}$ and gas catalyzed with $\mathrm{NH}_{3}$. For solution catalyzed synthesis we use three preparations: $A-2 \mathrm{wt} \%$ CTAB or CTAS, $[\mathrm{NaOH}]=0.174 \mathrm{M},[\mathrm{Si}]=0.40 \mathrm{M} ; \boldsymbol{B}-4 \mathrm{wt} \% \mathrm{CTAB}$ or CTAS, $[\mathrm{NaOH}]=$ $0.33 \mathrm{M},[\mathrm{Si}]=0.76 \mathrm{M} ; \mathrm{C}-8 \mathrm{wt} \% \mathrm{CTAB}$ or CTAS, $[\mathrm{NaOH}]=0.60 \mathrm{M},[\mathrm{Si}]=1.38 \mathrm{M}$. For gas-catalyzed synthesis, we used $C T A B$ and silica in the concentrations listed in methods $A, B$ and $C$ and designate these preparations $D, E$, and $F$, respectively. In all preparations the solvent is a mixture of water and a cosolvent. We define $r$ as the weight percent cosolvent in the mixture. The Si:surfactant ratio is 7.6 for all preparations.

C.2 Measurements - X-ray diffraction, light scattering, ${ }^{29} \mathrm{Si} \mathrm{NMR}$, and SEM/TEM data were collected and treated as described in sections II.C.2-5. 
C.3 Gas Adsorption Measurements - $\mathrm{N}_{2}$ sorption isotherms were collected at $77 \mathrm{~K}$ on calcined films that had been deposited on ST-cut quartz surface acoustic wave (SAW) devices. [34] Prior to making measurements the system was outgassed at $\sim 160^{\circ} \mathrm{C}$ for $2 \mathrm{~h}$. Mass flow controllers were used to vary the partial pressure of nitrogen $\left(\mathrm{P} / \mathrm{P}_{\mathrm{o}}\right)$ in a nonadsorbing helium carrier stream from $\sim 3 \%$ to $\sim 100 \%$ for the adsorption branch and $\sim 100 \%$ back to $\sim 3 \%$ for the desorption branch. The device was oscillated at $\sim 97 \mathrm{MHz}$ and the change in frequency with changing $\mathrm{P} / \mathrm{P}_{\mathrm{o}}$ was monitored and converted to a change in adsorbed mass. The sensitivity can be as great as $\sim 80 \mathrm{pg} / \mathrm{cm}^{2}$, where $\mathrm{cm}^{2}$ refers to the active area of the SAW covered by film (in our case $\sim 0.15 \mathrm{~cm}^{2}$ ). The BET model is then used to determine surface area, assuming $16.2 \AA^{2}$ for the area of a nitrogen molecule on the surface.

\section{Results and Discussion}

We explored a number of strategies to make thin films before developing gas-catalyzed synthesis; reviewing these efforts gives insight into the benefits of this approach. In many cases the film morphologies we obtained were unexpected, even bizarre. Before we were able to form thin films, however, we had to address several problems with the coating solutions.

D.1 Solution Homogeneity and Reaction Kinetics - Our first efforts addressed making a homogeneous solution from which to dip- or spin-coat films. In order to make homogeneous solutions the reagents should be molecular (i.e. alkoxides) and must all be dissolved prior to product formation. The key is to find a solvent system that is hydrophobic enough to solubilize the alkoxide, yet hydrophilic enough to allow the surfactant to act as a chemical dipole, which is essential if it is to act as a template. [33] The solvent system must contain some water to promote the hydrolysis and condensation of the alkoxide. We found that a wide variety of cosolvents 


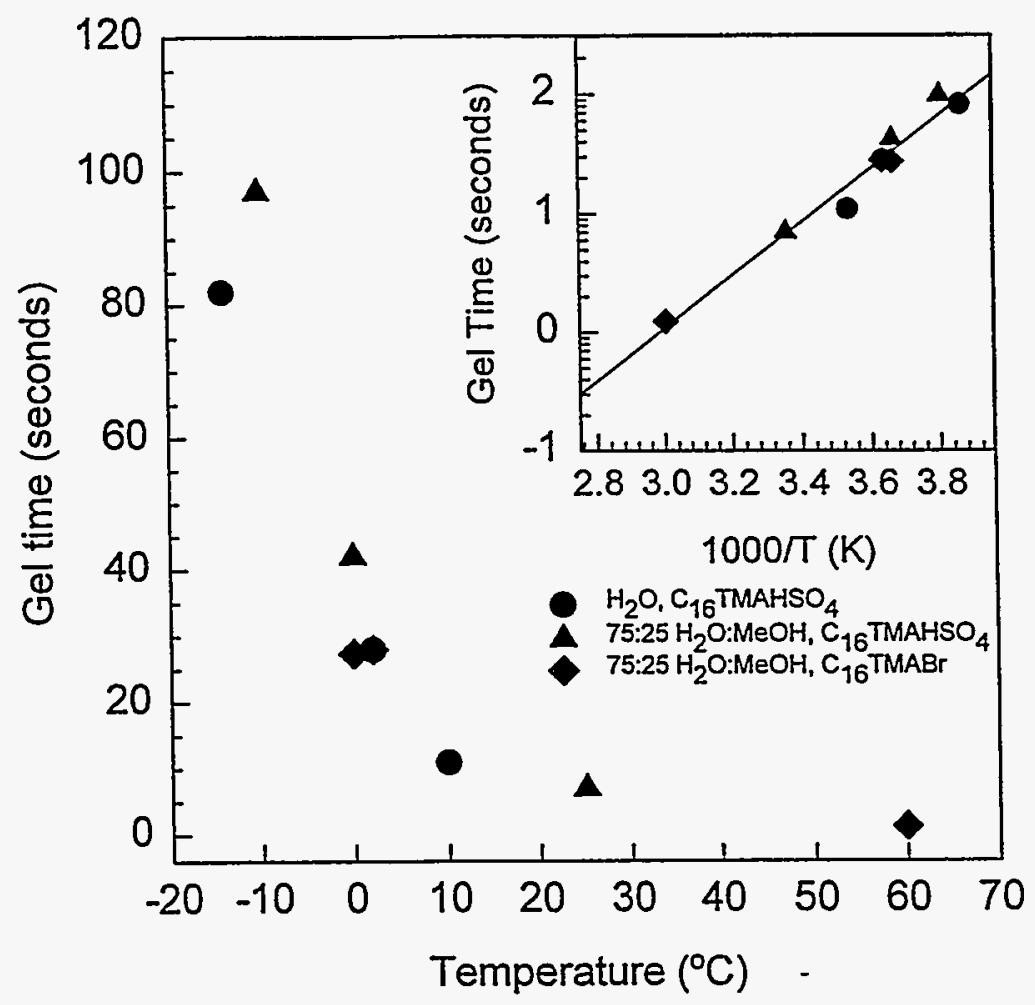

Figure 20. The gel time is strongly dependent on temperature and can be usefully described by an Arrhenius law. The rate limiting step in gel formation is the hydrolysis of silica.

combined with water meet these criteria, including, alcohols, glycols, amide, and polyethers.

The next problem to address was to speed the reaction kinetics. This is essential because when TEO [3,4] or non-molecular sources of silica $[1,2,6]$ are used a wide variety of species are simultaneously present in the reaction mixture, such as, partially hydrolyzed alkoxides $\left(\mathrm{Si}\left(\mathrm{OCH}_{3}\right)_{4-x}(\mathrm{OH})_{x^{\prime}}\right.$ silicate oligomers, colloidal products, amorphous silica, free surfactant, and precipitated product. Obviously this is not an optimal mixture from which to make pure, continuous periodic mesoporous thin films. Fortunately, the kinetics of product formation are accelerated by $\sim 2$ orders of magnitude when TMOS is used as the silica source instead of TEOS. In fact, periodic mesoporous silica forms within 5-7 seconds at room temperature with TMOS, Fig. 20, and aggregation and gelation occur in less than 1 second. This nearly instantaneous transformation from homogeneous solution to periodic 
product effectively eliminates the broad distribution of species found when TEOS is the silica source.

Forming products with TMOS eliminates the problems of wide particle size distributions and uneven agglomeration found with precipitated colloidal particles. The TMOS-derived product forms aggregated colloidal particles that are 20-500 nm diameter. The particles form a continuous gel network in which the particle-particle contact area is minimal, which provides ready access to the intraparticle porosity for adsorbing gases.

\section{D.2 Spin Coating Premixed Water:Cosolvent Solutions - As shown in Fig.} 20, the time to form a product is increased as the temperature is decreased. We reasoned that a homogeneous solution could be deposited onto a substrate and spun before it gelled. The shear forces during spinning provide a liquid layer of essentially constant thickness, except at the extreme periphery of the substrate. The speed at which the substrate is spun thus fixes the reaction volume and the final thickness of the film.

Formamide: In our first experiments we mixed the reagents as if to make a bulk gel and spin coated these onto a substrate. We used Preparation A with $\mathrm{CTAB}$ and a substantially nonaqueous, $r=90 \%$ formamide solvent (water:Si ${ }^{\circ} h=12.9$ ). The gel point was increased to about 30 seconds by cooling the reactants to $0{ }^{\circ} \mathrm{C}$ before adding the TMOS. Quaternary ammonium surfactants with bromide or chloride anions phase separate at low temperature, but we found that CTAHS does not. When using CTAHS we were careful to add enough extra $\mathrm{NaOH}$ to titrate the $\mathrm{HSO}_{4}^{-}$ion and maintain a nominal $\mathrm{pH}$ of 13 after titration. If these reactants are allowed to form a bulk gel, XRD data (Fig. 21) show that the hexagonal mesophase forms, with a lattice periodicity of about $40 \AA$.

To make the thin films, the basic surfactant solution was stored on ice, as was the TMOS. We injected the TMOS into the surfactant solution and spin coated the silicon or silica substrate before the solution gelled. The 


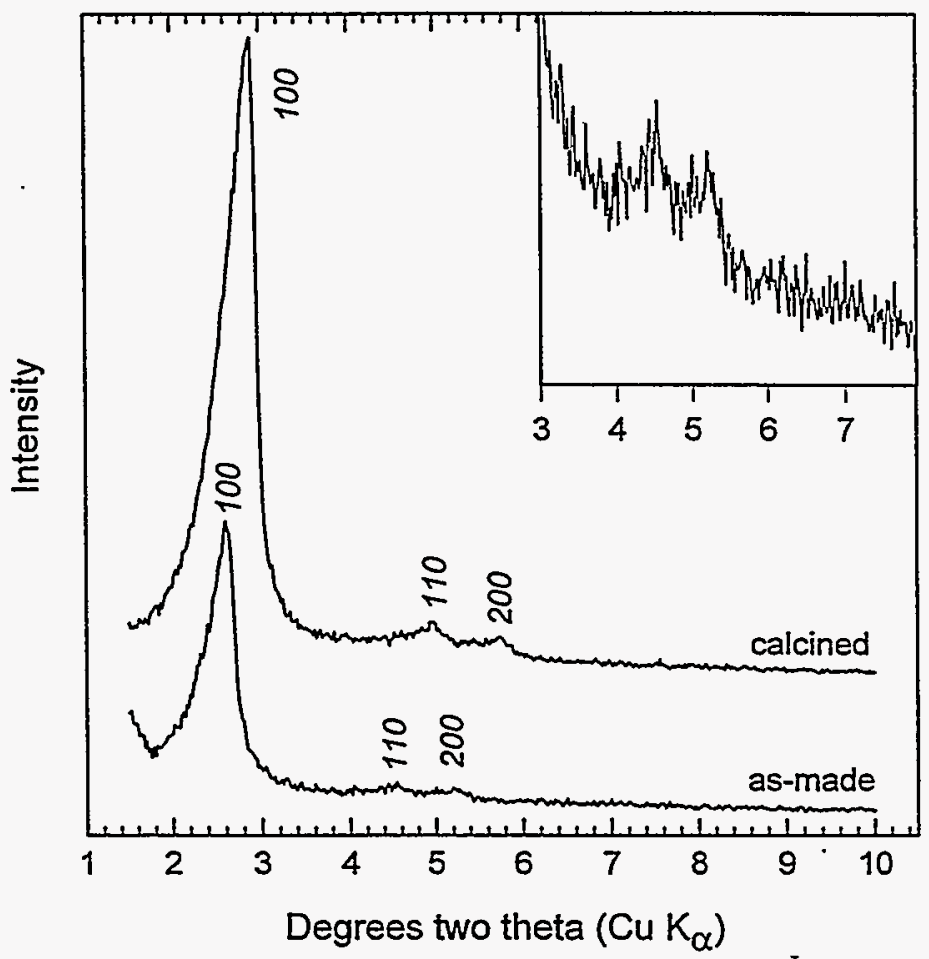

Figure 21. X-ray diffraction data for an as-made mesoporous bulk gel made using Preparation $A$ with CTAB and $r=90 \%$ formamide (h $» 13$ ), and the same sample calcined at $550^{\circ} \mathrm{C}$ in $\mathrm{O}_{2}$ for 10 h. The as-made gel has a hexagonal lattice constant of $\sim 40.0 \AA$ compared to $\sim 36.5 \AA$ for the calcined sample. The inset shows the higher order reflection region for the as-made sample.

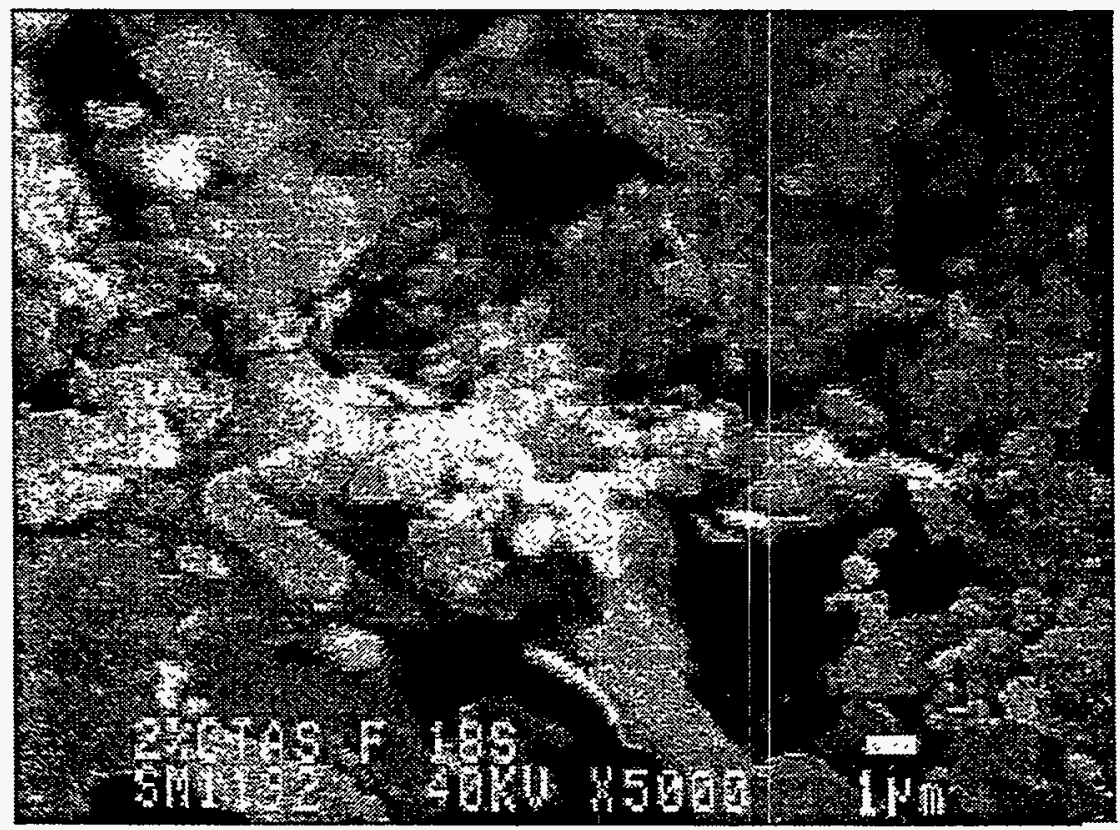

Figure 22. Scanning electron micrograph of a thin film made by spin coating premixed reactants (method A with CTAS and $r=90 \%$ formamide). Solution was mixed at $0{ }^{\circ} \mathrm{C}$ and deposited on a room temperature substrate. Coverage is non-uniform and consists only of particle aggregates nucleated in solution. 
resulting films appeared uniform to the unaided eye, but optical and scanning electron microscopies revealed that coverage was incomplete and spotty. We varied the spinning speed from 500 to $9000 \mathrm{rpm}$ and time at which we spun the solution (before, during, and after formation of the product), but always obtained non-uniform coverage, Fig. 22.

We examined the effect of leaving out the water from the reactant mixture $(\mathrm{r}=100 \%)$. The idea being that after the thin film was spun, moisture from the atmosphere would diffuse into the solution and start the reaction. This did not lead to improved results. However, substantially increasing the relative humidilty by heating the initial film overnight in steam $\left(100^{\circ} \mathrm{C}\right)$ led to periodic mesoporous films that show one peak at $\sim 30 \AA$ in the XRD spectrum. Unfortunately there is also a significant amount of unreacted surfactant in the film. Higher temperatures and longer reaction times may improve this approach.

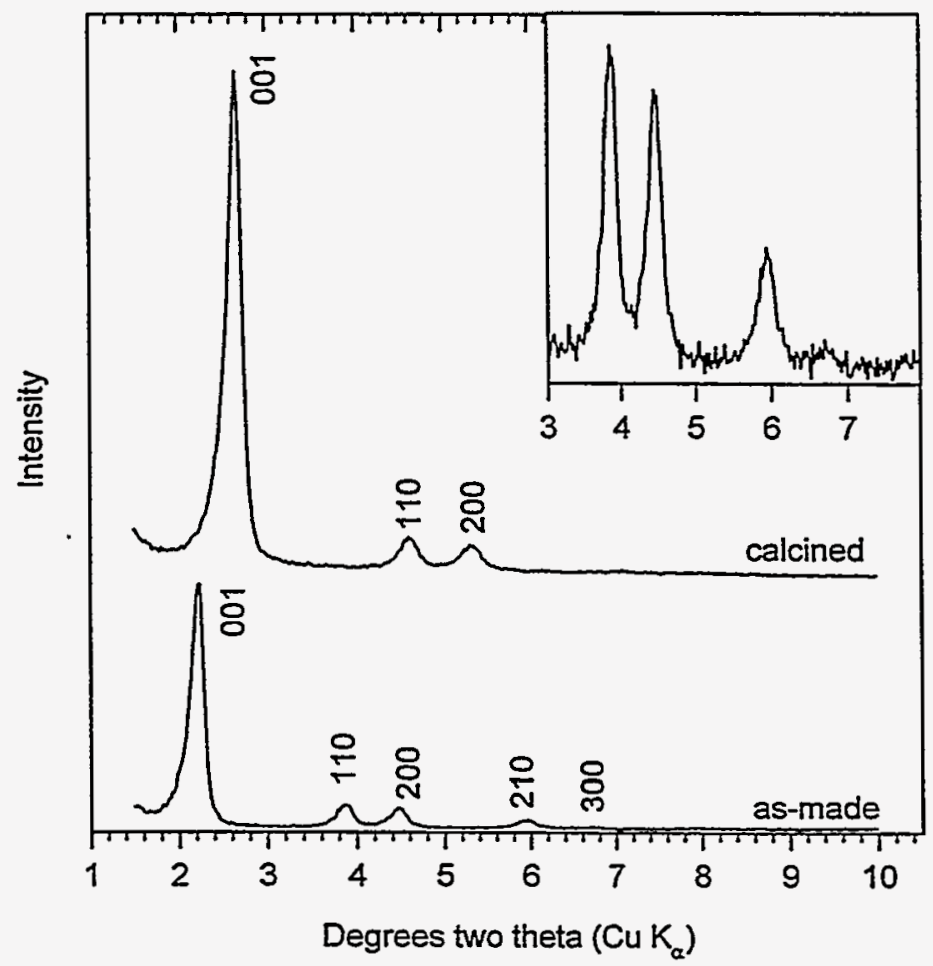

Figure 23. X-ray diffraction data for an as-made mesoporous bulk gel made using Preparation $A$ with $C T A B$ and $r=25 \%$ methanol $(h » 97)$, and the same sample calcined at $550^{\circ} \mathrm{C}$ in $\mathrm{O}_{2}$ for 10 h. The as-made gel has a hexagonal lattice constant of $\sim 45.3 \AA$ compared to $\sim 38.4 \AA$ for the calcined sample. The inset shows the higher order reflection region for the as-made sample. The coherent scattering domain size for the as-made sample is $\sim 140 \mathrm{~nm}$. 
Methanol: Our next approach was to try a different mixed solvent system. Bulk samples made using method A with CTAB and $r=25 \%$ methanol always gave excellent long-range order (scattering domain sizes up to $140 \mathrm{~nm}$ ), Fig. 23. In an attempt to obtain better coverage, we doubled the reagent concentrations (method B) and used CTAS with $r=25 \%$ methanol.
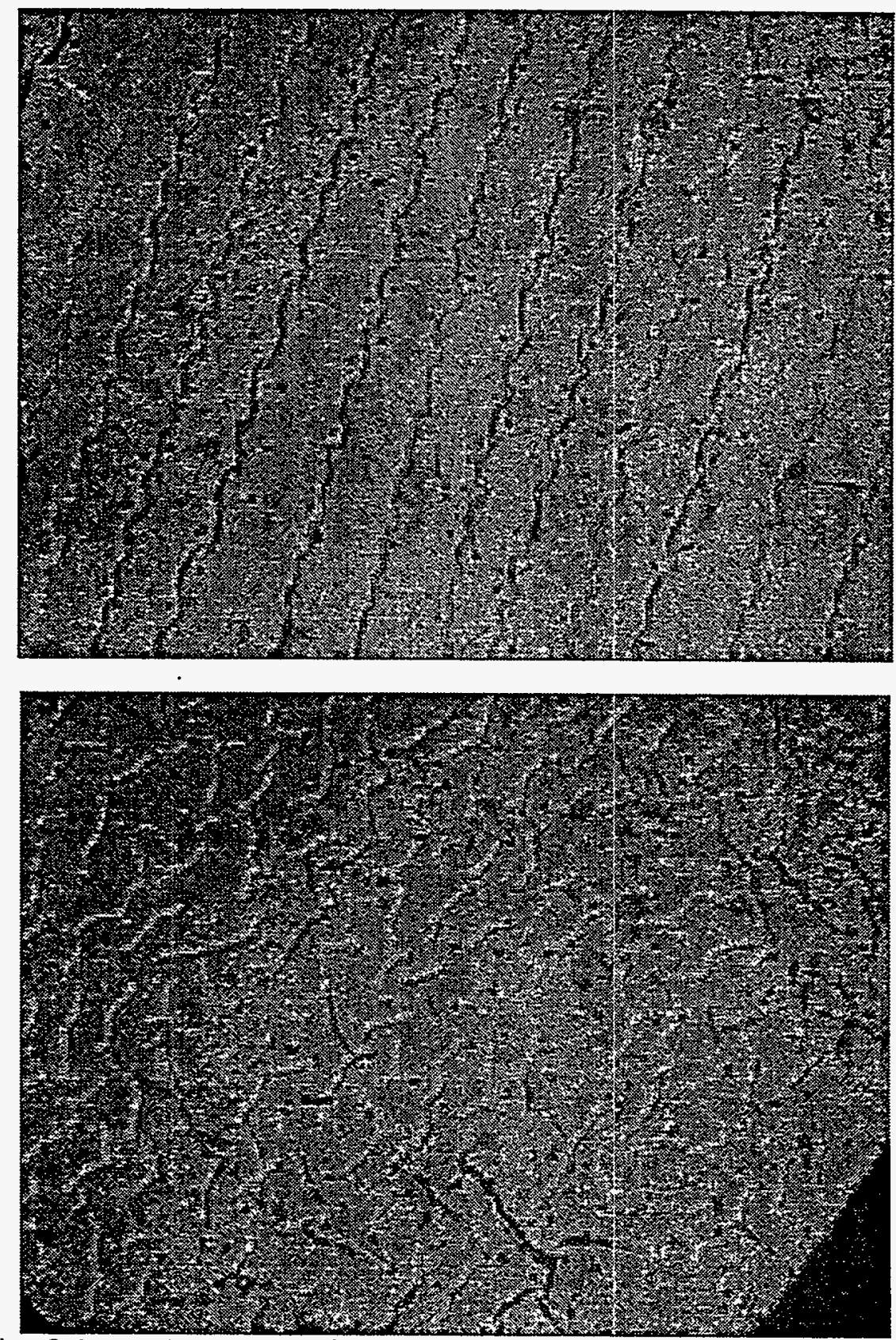

Figure 24(a). Spin coating premixed reactants (Preparation B with CTAFS and $x=25 \%$ methanol) gave bizarre tire tread type structures. The lines cannot be dissolved by common solvents and may form by the deposition of silica from convective rolls. (b). In other areas of the same coated substrate the lines form branched structures. 
These systems produced bizarre films. Fig 24a shows the parallel line structures obtained by spin coating. These lines cannot be dissolved by common solvents. In other areas of the same coated substrate, branched wiggley lines occur, Fig $\mathbf{2 4 b}$. Electron microscopy of these structures revealed finer detail, Fig. 25, but the actual mechanism of pattern formation remains enigmatic.

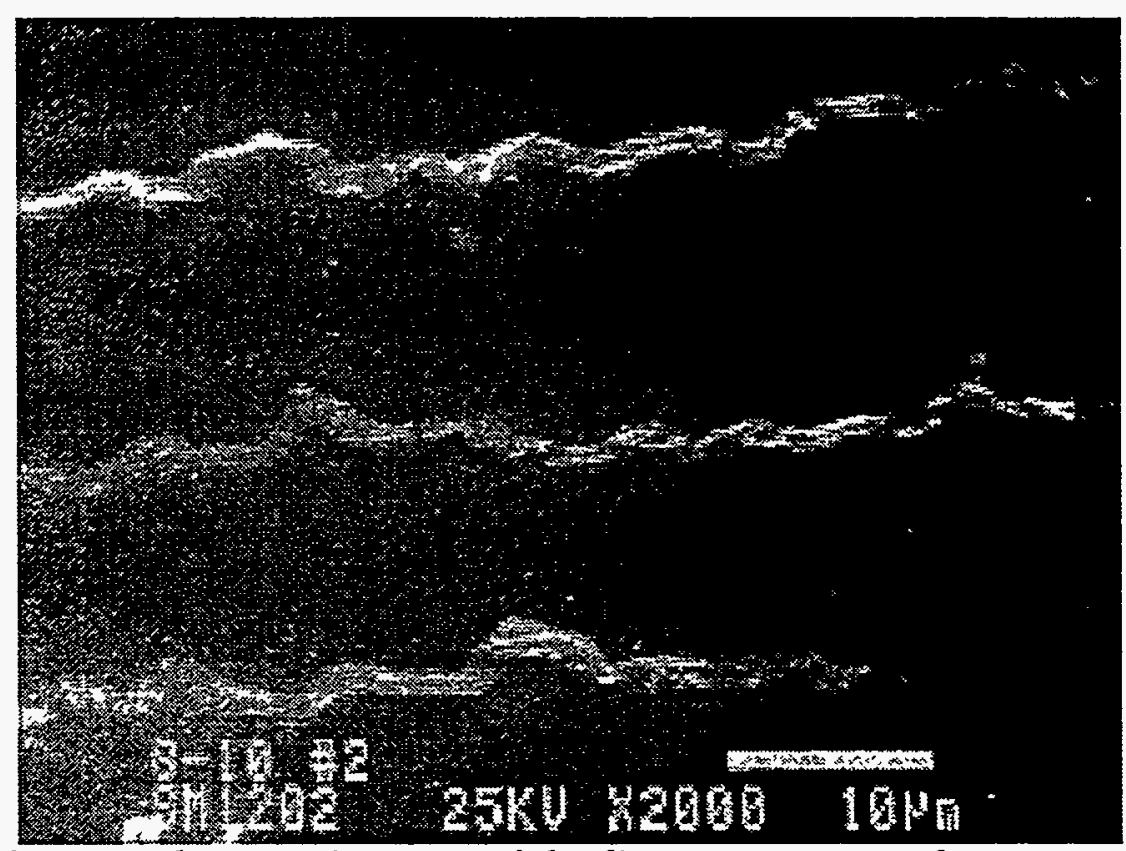

Figure 25. Scanning electron microscopy of the line structures reveal an apparently wound structure that may indicate the interaction of evolving structures with convective rolls.

We were able to consistently reproduce the line structures. We performed a control experiment by leaving out the TMOS; the CTAS solution formed beautiful Diffusion Limited Aggregates (DLA), Fig. 26, of which much has been written in the literature. [35] DLA clusters are produced when diffusing particles stick upon contact with the growing cluster, without the ability to restructure. These dendritic surfactant structures have a different morphology than structures formed in the presense of silica and they rinse away with methanol, so we suspected that the lines found in the presense of TMOS are some form of silica. SEM with EDX confirms that the lines contain silicon. 


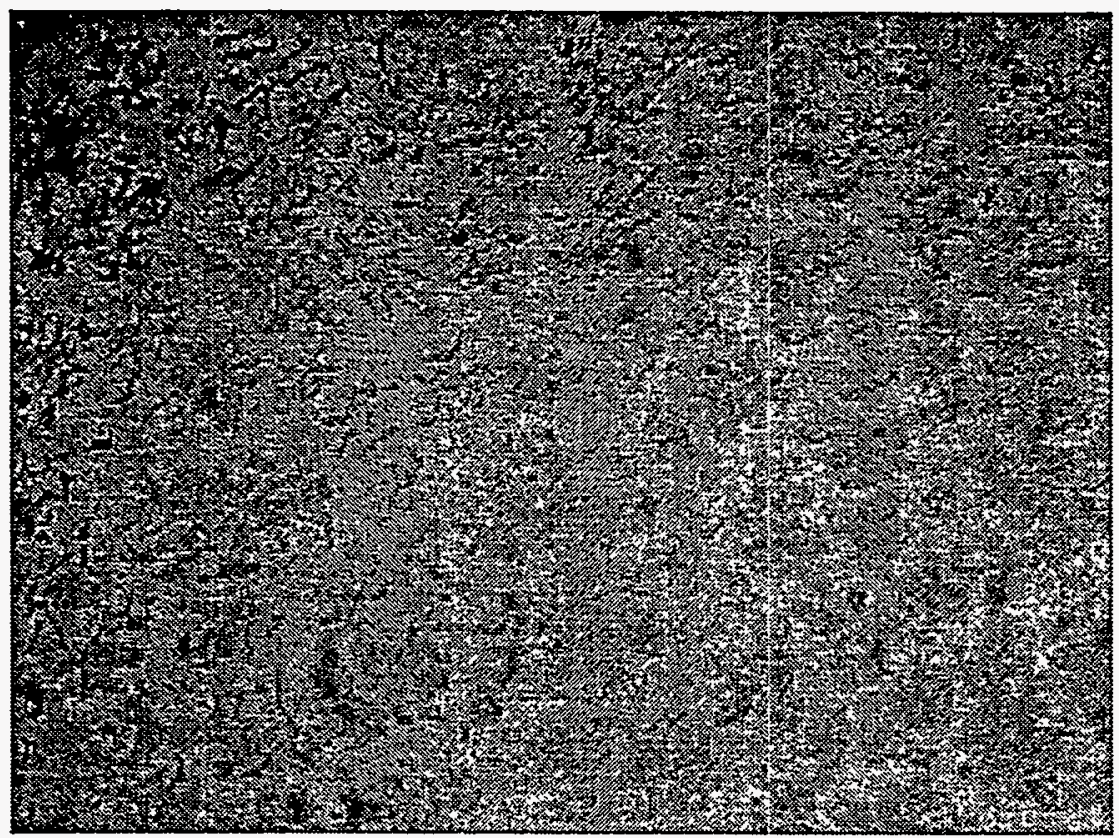

Figure 26. In an effort to gain some insight into the line structures produced using methanol as a cosolvent, we conducted a control experiment without TMOS. This has a tremendous effect on structure formation, producing fractal DLA clusters of CTAHS. These clusters rinse away with common solvents such as methanol, and do not appear when TMOS is added to the solution. The concentration of CTAS is $4 \mathrm{wt} \%$ in an alkaline $75: 25$ water:methanol solution.

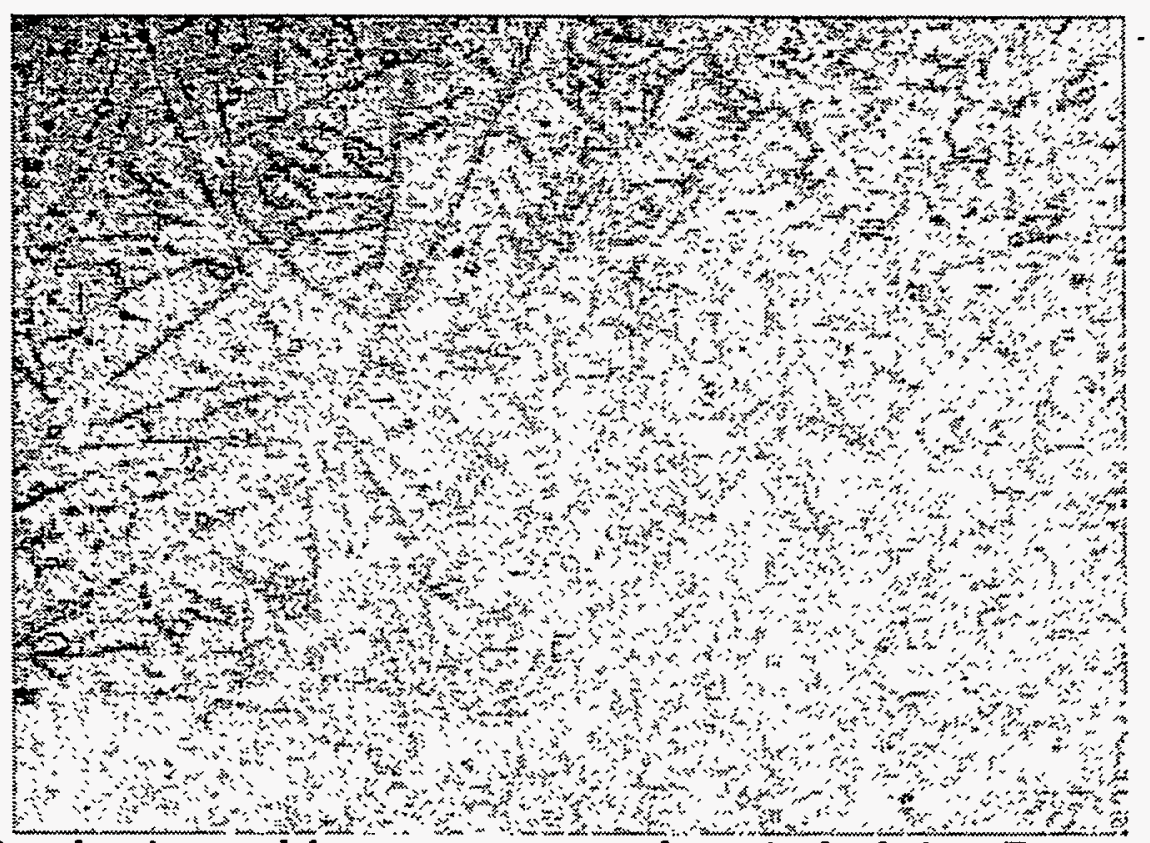

Figure 27. Sample spin coated from more concentrated premixed solutions (Preparation $C$ with CTAHS and $r=25 \%$ methanol) did not give better surface coverage, but produced sparse branched structures. Without TMOS we again obtained DLA clusters from $8 \%$ CTAS.

With 8\% CTAHS (method C) the results were somewhat different. Without TMOS we again obtained DLA clusters. With TMOS we obtained 
the sparse branched structures in Fig. 27, which again have poor surface coverage.

To determine the effect of the surfactant anion on structure, we used Preparation $B$ with CTAB and $r=25 \%$ methanol and cooled the solutions on ice to just above the phase boundary $\left(-10^{\circ} \mathrm{C}\right)$. Spin coating these solutions led to the particle/line-like structures in Fig. 28a, which again were not dissolved by an alcohol rinse. Some coatings contained fractal-like aggregates, Fig. $\mathbf{2 8 b}$.

From these early studies we realized that the rapid kinetics observed for the TMOS system are advantageous in that we avoid a wide distribution of species in solution, and, as we generally use aqueous-based solutions that have a high surface tension, we can form films before the solution dewets. Nonetheless, without strict temperature control and precise timing the kinetics are problematic in that the exact instant of product formation is difficult to predict accurately enough to get continuous films. Thus a method is needed to predictably delay the product formation until the coating solution is on the substrate in the appropriate thickness.

D.3 Gas-Catalyzed Thin Film Synthesis - In the work thus far described, we attempted to control the rate of reaction simply by reducing the temperature; however the rates of hydrolysis and condensation of the TMOS are not only sensitive to temperature, but to $\mathrm{pH}$. We decided to try to form a stable coating solution at $\mathrm{pH}=7$ by leaving out the base and then diffusing the catalyst to the substrate in the form of ammonia gas. The idea is that we can deposit the coating solution on a substrate, spin or drain it to the desired thickness, and by turning on the ammonia ( $\mathrm{pH}$ switch), rapidly form the product.

Bulk Products: To confirm that we could form periodic mesoporous silica by this approach, we made bulk products by exposing the neutral reaction solution to ammonia gas (Preparation D). X-ray diffraction clearly shows that this process leads to periodic mesoporous silica, Fig. 29. Furthermore, the product forms in the top few millimeters of the static liquid within $\sim 10$ s. Thus, by dip coating the neutral solution onto silicon substrates 

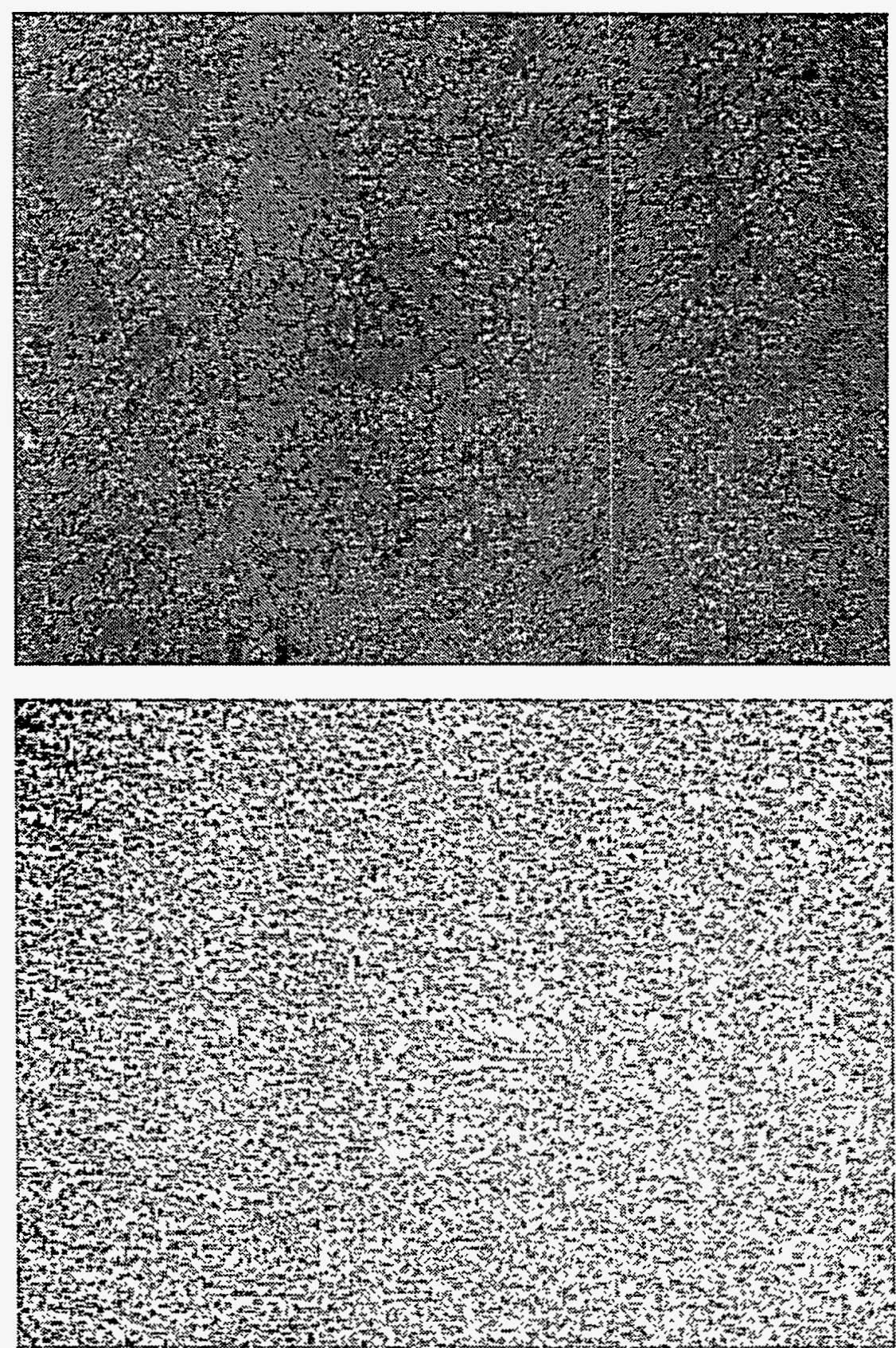

Figure 28(a). Samples spin coated using premixed solutions (Preparation B with CTAB and $r=$ $25 \%$ methanol) gave the particle/line-like structures that survived a solvent rinse, which indicates that they are silica based. (b). Some coatings contained fractal-like aggregates that probably formed in bulk solution before spin casting.

(method $\mathrm{F}$ with CTAB and $\mathrm{r}=25 \%$ methanol) and then gassing with ammonia, we were able to make films with diffraction patterns characteristic of periodic mesoporous silica, Fig. 30 .

Methanol ( $r=25 \%$ ): Our first attempts at gas catalyzed synthesis were with dip coating using Preparation D. Macroscopically the films appeared to 


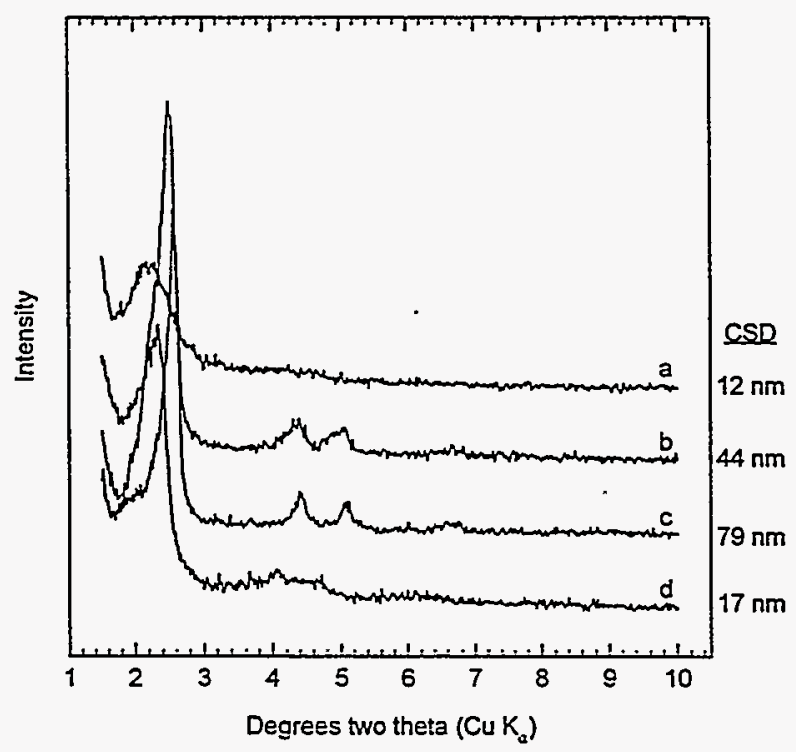

Figure 29. X-ray diffraction data from bulk samples made by Prep. $D$ with $r=50 \%$ ethylene glycol (a) and $r=25 \%$ methanol (b-d) solutions indicate hexagonal structure. The coherent scattering domain (CSD) size depends on cosolvent, agitation, and solution temperature.

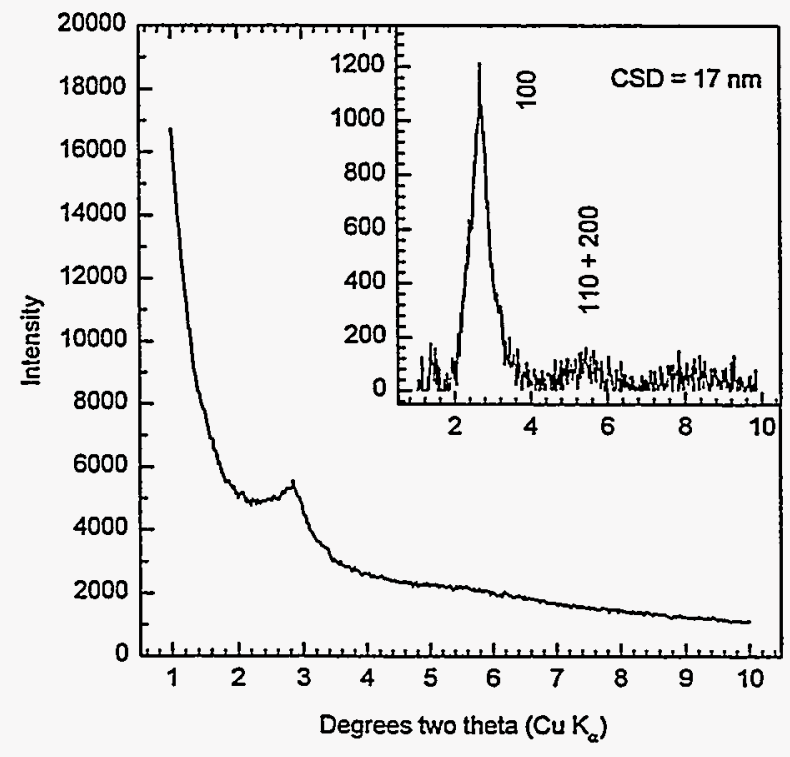

Figure 30. X-ray data from a thin film sample on silicon made by Prep. F with $r=25 \%$ methanol indicate hexagonal structure. Inset is the pattern with the background subtracted. The CSD size calculated from the 100 reflection is $17 \mathrm{~nm}$. The convolution of the 110 and 200 reflections is similar to that seen in the bulk sample with a $17 \mathrm{~nm}$ CSD. 


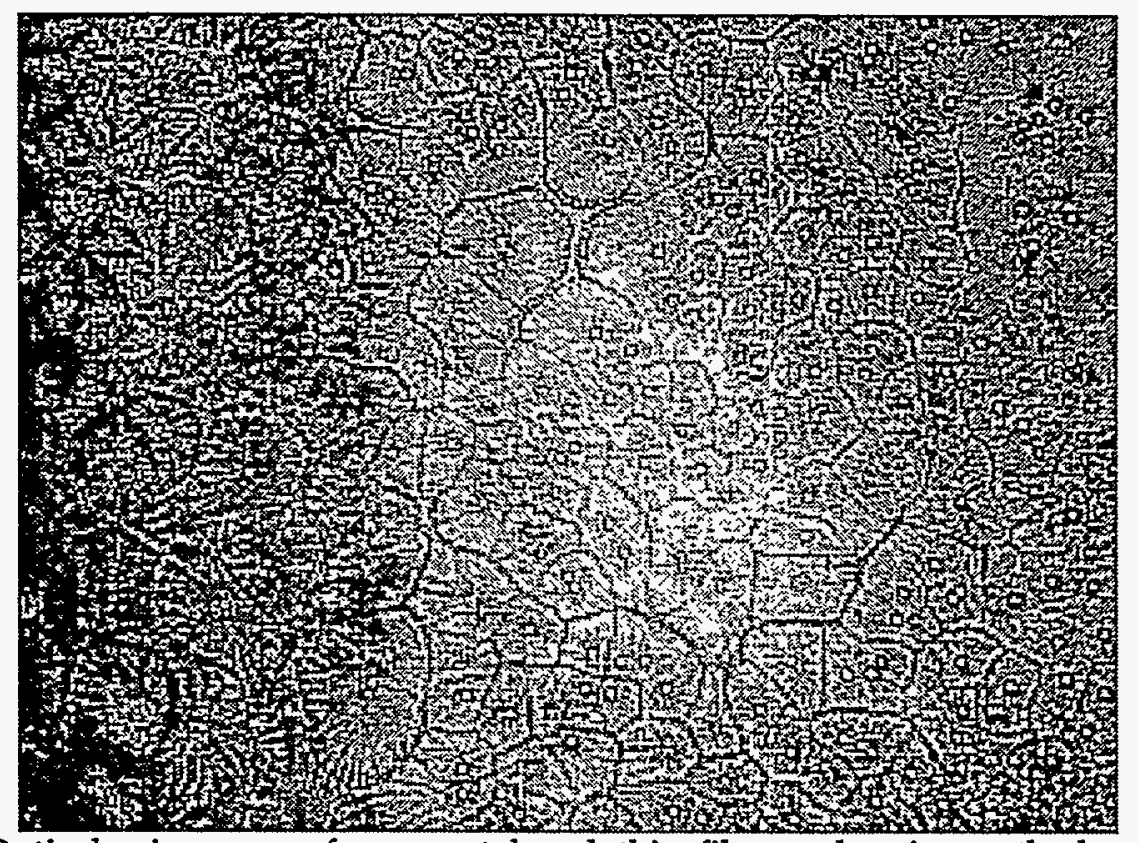

Figure 31. Optical microscopy of a gas-catalyzed thin film made using method with $r=25 \%$ methanol reveals polygonal tessellation that we believe is due to silica deposition from convection cells. The convection cells are driven by the volatility and heat of vaporization of methanol.

have uniform thickness, but optical microscopy revealed the peculiar pattern of Fig. 31. We investigated the cause of the formation of this pattern by mixing methanol with an aqueous suspension of $1 \mathrm{~mm}$ polystyrene latex spheres that we squirted onto a microscope slide. Under an optical microscope the latices allowed visualization of complex convection rolls and cells, the formation of which are driven by the volatility and heat of vaporization of methanol. At first the convection was dominated by rolls, but as the system became more concentrated, the rolls started to show instabilities along their boundaries. These periodic instabilities grew in amplitude, then pinched the roll into polygonal cells that eventually dissipated as the methanol evaporated away.

We believe the pattern in Fig. 31, and perhaps the wiggley line pattern discussed earlier, is due to deposition of silica from convective cells. If this is indeed the case there are two reasonable approaches to the problem; switch to 

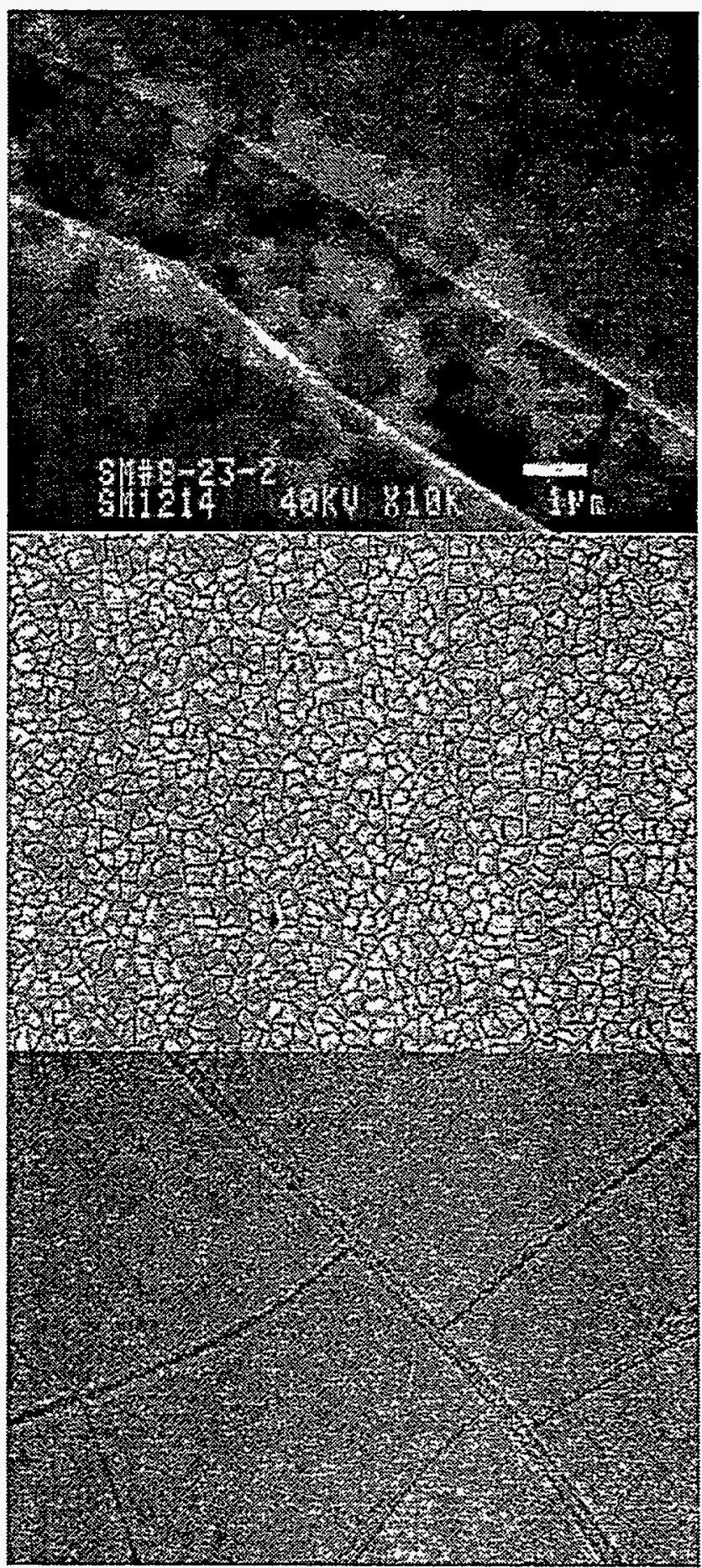

Figure 32(a). A gas-catalyzed thin film made using Preparation D with $r=50 \%$ formamide shows uniform coverage, but with some coarse cracking. (b) The cracking becomes more pronounced and occurs on a finer scale after a methanol wash. (c) Electron microscopy reveals that the street cracks prior to washing comprise thin layer above $250 \mathrm{~nm}$ aggregated particles. 
a less volatile solvent system, such as, water/formamide, or control the methanol fugacity in the environment to which the substrate is exposed during coating.

Formamide ( $r=50 \%$ ): Gas catalyzed synthesis using Preparation $D$ gave the uniform films shown in Fig. 32a; the street pattern of cracks is due to film shrinkage during drying. After a methanol wash the film shrinkage problems became more acute Fig 32b. Electron microscopy of the films revealed the formation of clustered $250 \mathrm{~nm}$ particles beneath a very thin continuous layer, Fig 32c. Most of the cracking problems are probably because the films are too thick. The films were pulled from solution more slowly to decrease thickness, but because of the high viscosity the solution drained slowly, and also dewetted, tending to give spotty coverage.

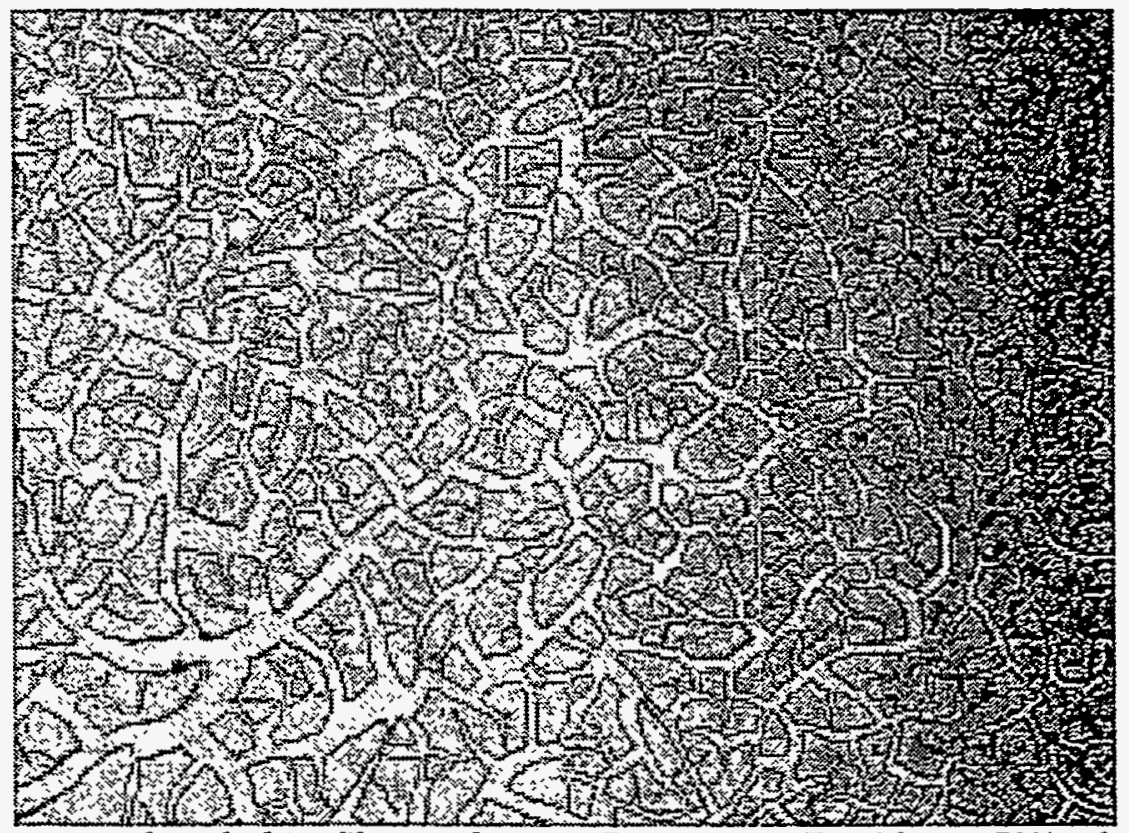

Figure 33. A gas-catalyzed thin film made using Preparation D with $r=50 \%$ ethylene glycol produced a uniform thin film that shrank badly after a methanol wash, producing a marvelous pattern reminiscent of bronchi. The change in the characteristic dimension of the pattern is due to varations in the film thickness; thinner films exhibit fracture on a finer scale.

Ethylene glycol ( $r=50 \%$ ): Gas catalyzed synthesis using Prepartion A gave a uniform film that shrank severely upon rinsing with methanol, creating the marvelous fragmentation pattern in Fig. 33, reminiscent of the recursive bronchi in Mandelbrot's The Fractal Geometry of Nature. [36] We 


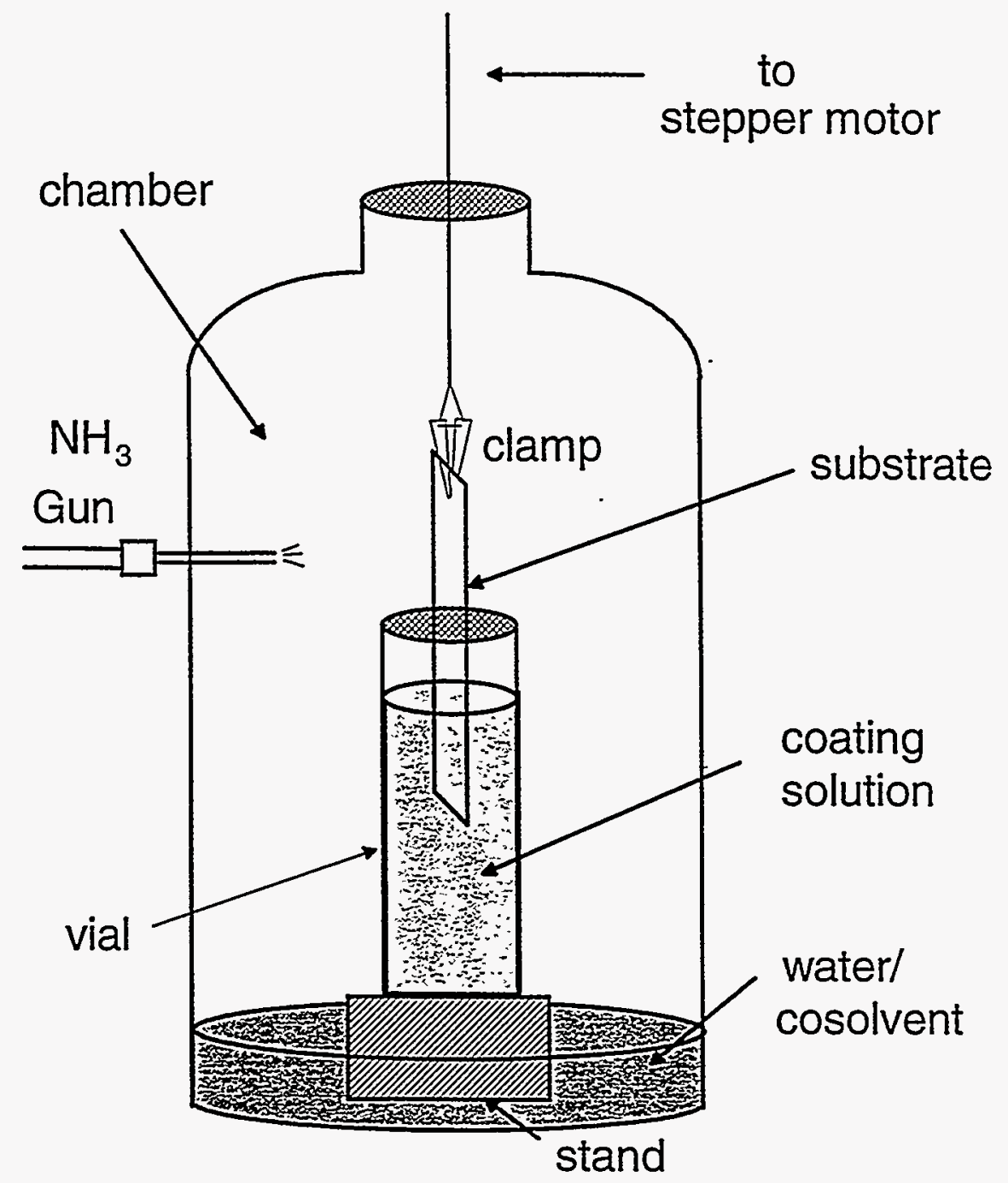

Figure 34. Schematic of the environmental chamber used for dip coating.

found that ethanol washes minimize film cracking problems, so we adopted this procedure in further studies. Again the high viscosity of the coating solution gave coverage, cracking, and uniformity problems.

Methanol ( $r=25 \%$ ) in an environmental chamber: Because of the viscosity and coverage problems with formamide and ethylene glycol, we decided to explore the second approach to eliminating the formation of convection cells with methanol. We built a simple environmental chamber, Fig. 34, for dip coating substrates and catalyzing product formation with ammonia gas. Into this chamber we placed a small dish of the $r=25 \%$ 
water:methanol solvent, thus insuring the fugacity of the methanol in the coating matched that in the chamber. Because the net evaporation of methanol is essentially zero in this case, we were able to produce uniform thin films that did not convect using method E, Fig. 35.

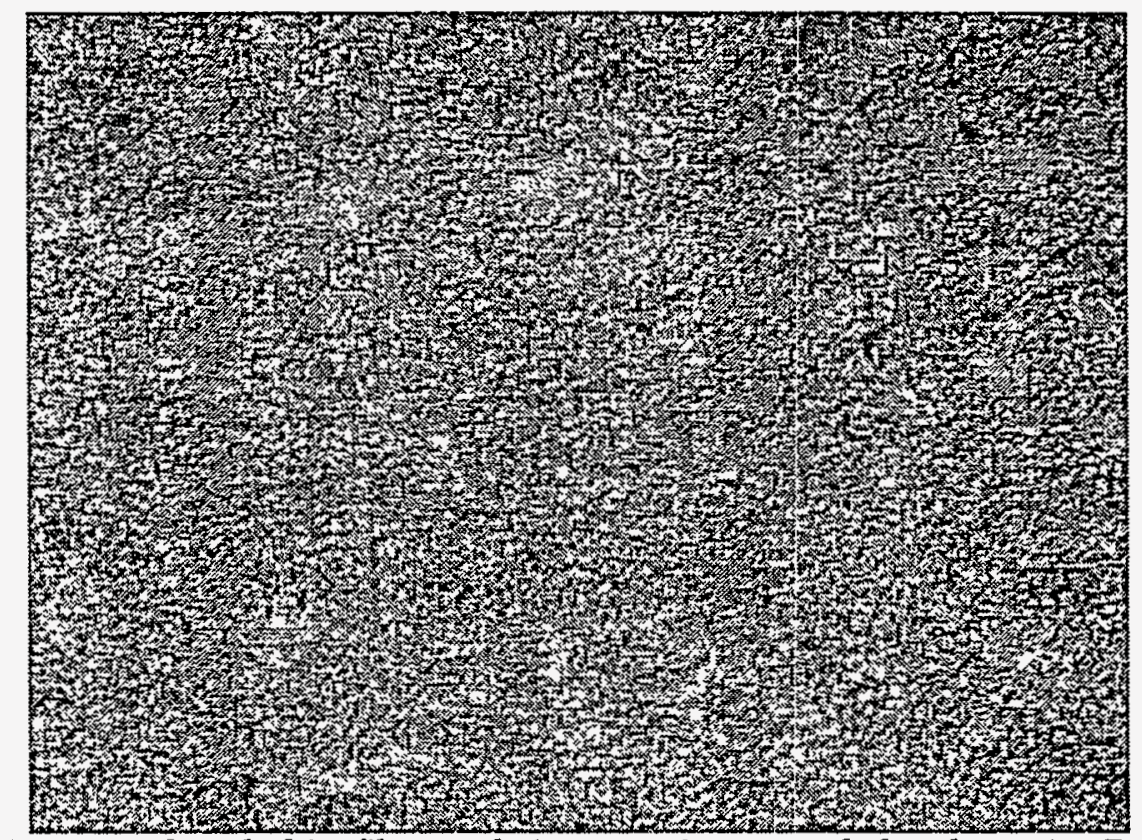

Figure 35. A gas-catalyzed thin film made in an environmental chamber using Preparation D with $r=25 \%$ methanol produces uniform thin films because the net evaporation of methanol is essentially zero in this case.

Chemistry of the neutral coating solution: After solving the convective problems with the environmental chamber, we focused on the chemistry of the $r=25 \%$ methanol coating solutions. The neutral coating solution with $8 \% \mathrm{CTAB}$ and $[\mathrm{Si}]=1.38 \mathrm{M}$ remains clear for $\sim 90$ minutes before a transparent or translucent chemical gel forms. Thus we wanted to determine the optimal time at which to dip the solution. We examined the evolution of silicate and surfactant species with static light scattering, dynamic light scattering, and ${ }^{29} \mathrm{Si} N \mathrm{NMR}$.

The NMR measurements, Fig. 36, show that in the $[\mathrm{Si}]=1.38 \mathrm{M}$ solution, $Q^{0}{ }_{10 H}$ species form after $\sim 10$ minutes, $Q^{1}$ species form after $\sim 30$ minutes, $Q^{2}$ species begin to form after $\sim 60$ minutes, and $Q^{3}$ and $Q^{4}$ species begin to form after 70 minutes. From light scattering measurements, Fig. 37, we see that the species in solution - micelles, and partially hydrolyzed 


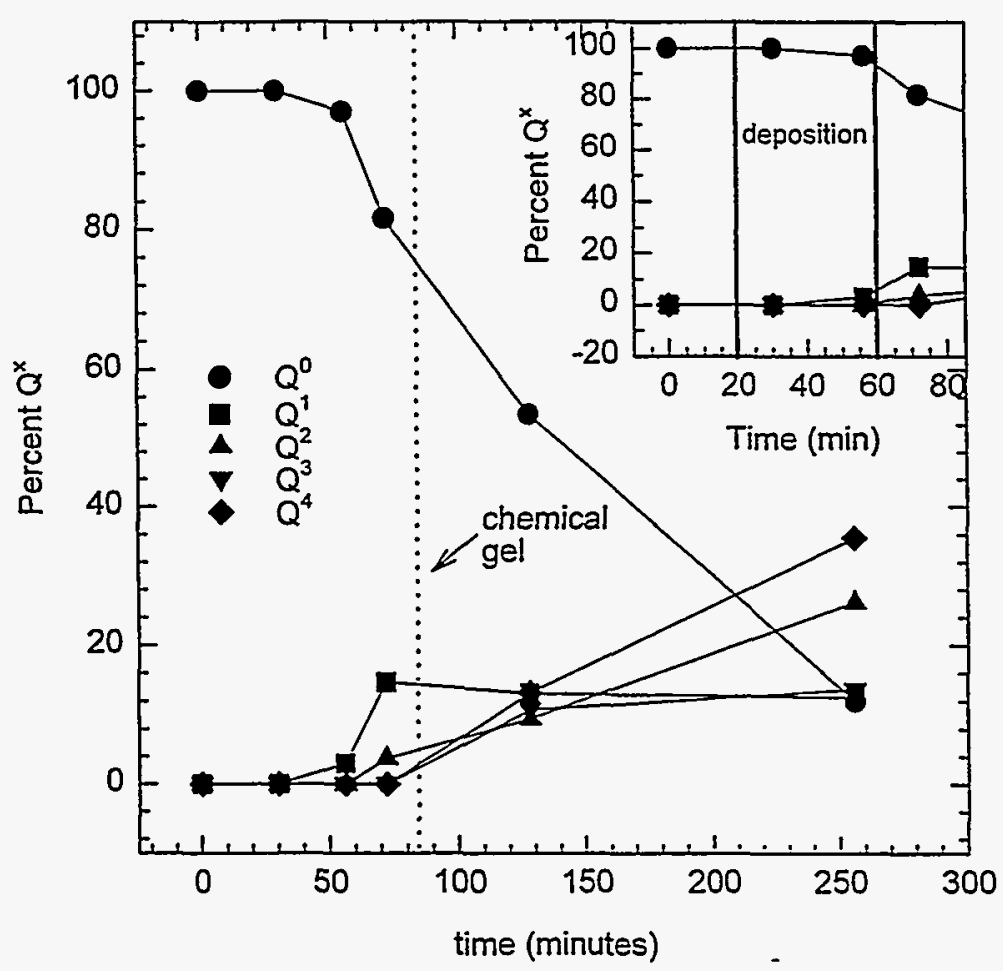

Figure 36. ${ }^{29} \mathrm{Si} N M R$ data for a neutral solution that was $8 \mathrm{wt} \% \mathrm{CTAB},[\mathrm{Si}]=1.32 \mathrm{M}$, and $\mathrm{r}=25 \%$ water:methanol show the evolution of $\mathrm{Si}-\mathrm{O}-\mathrm{Si}$ bridging oxygens. These data show that siloxane bonds do not form until $\sim 30$ minutes $\left(t / t_{\text {gel }} \gg 0.3\right.$ ). The inset shows that the optimal deposition occurs just prior to the first siloxane bond formation to just after the formation of $Q^{3}$ species. The hydrolysis of the first methoxy group occurs after about 10 minutes, which corresponds well with the observed time at which the solutions begins to wet the substrate.

alkoxide monomers and oligomers - do not scatter significantly for about $1 \mathrm{~h}$ in a 1.38 $\mathrm{M}$ silica solution. The chemical gels that form at 90 minutes are amorphous in $\mathrm{X}$-ray diffraction and therefore are not a desired product. Thus we find the optimal time window to deposit the coating solution is between 10 and 60 minutes. In this range the silicate precursor have begun to hydrolyze so they wet more hydrophilic substrates, and the silicate species are still small so they do not frustrate the transformation to PMS.

D.4 Optimizing Film Thickness and Coverage - Gas-catalyzed synthesis in an environmental chamber leads to uniform periodic mesoporous silica films, but the film thickness and coverage have to be optimized. The ultimate thickness of the film is determined by the coating conditions, that is, spin speed or withdrawal rate of the substrate, along with the initial silica 


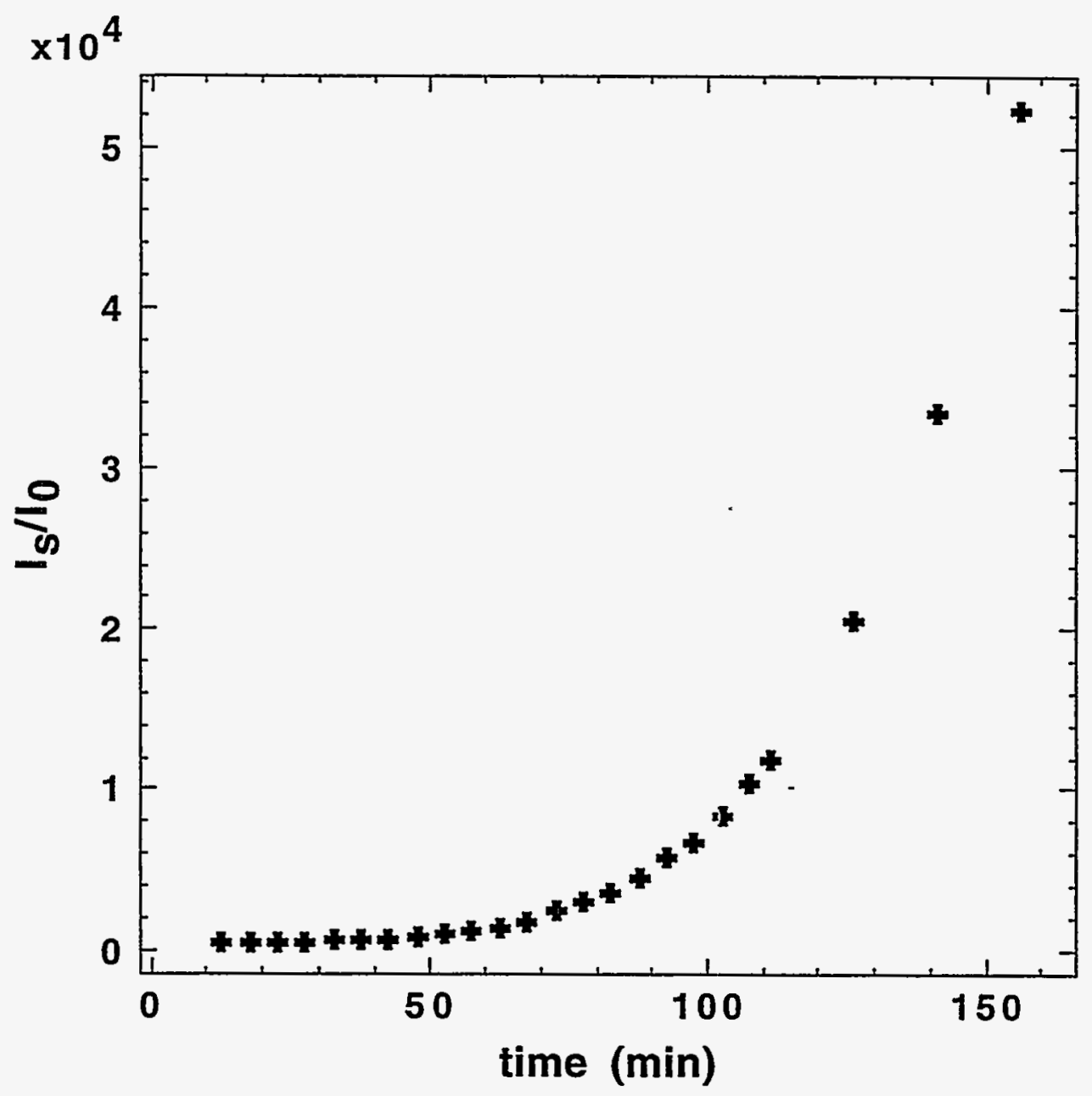

Figure 37. Light scattering intensity as a function of time for a solution made from $8 \mathrm{wt} \% \mathrm{CTAB}$, $[\mathrm{Si}]=1.38 \mathrm{M}$, and $\mathrm{r}=25 \%$ water:methanol indicate an incubation time during which hydrolysis occurs but particle formation is negligible. Data on a 2 wt\% CTAB, $[\mathrm{Si}]=0.40 \mathrm{M}$, and $\mathrm{r}=50 \%$ water:formamide solution show an $\sim 50$ minute incubation time; quasielastic light scattering demonstrates that after 10 minutes the particle radius is only about $5.5 \mathrm{~nm}$, which is about twice the initial micelle radius.

concentration. Conditions that lead to films that are less than $\sim 1 \mathrm{~mm}$ thick are optimal in that films do not crack when washed or calcined at $500^{\circ} \mathrm{C}$.

The coverage depends on the initial concentration of silica in the coating solution. Figs. 38a \& $b$ show the difference in coverage for a film made from a $[\mathrm{Si}]=0.40 \mathrm{M}$ solution versus one made from a $[\mathrm{Si}]=1.38 \mathrm{M}$ solution. The coverage is continuous and complete for the latter, whereas it is not continuous for the former. In general we find that for continuous 
coverage it is best to use solutions that have an initial silica concentration of $\sim 1.3 \mathrm{M}$ or greater.
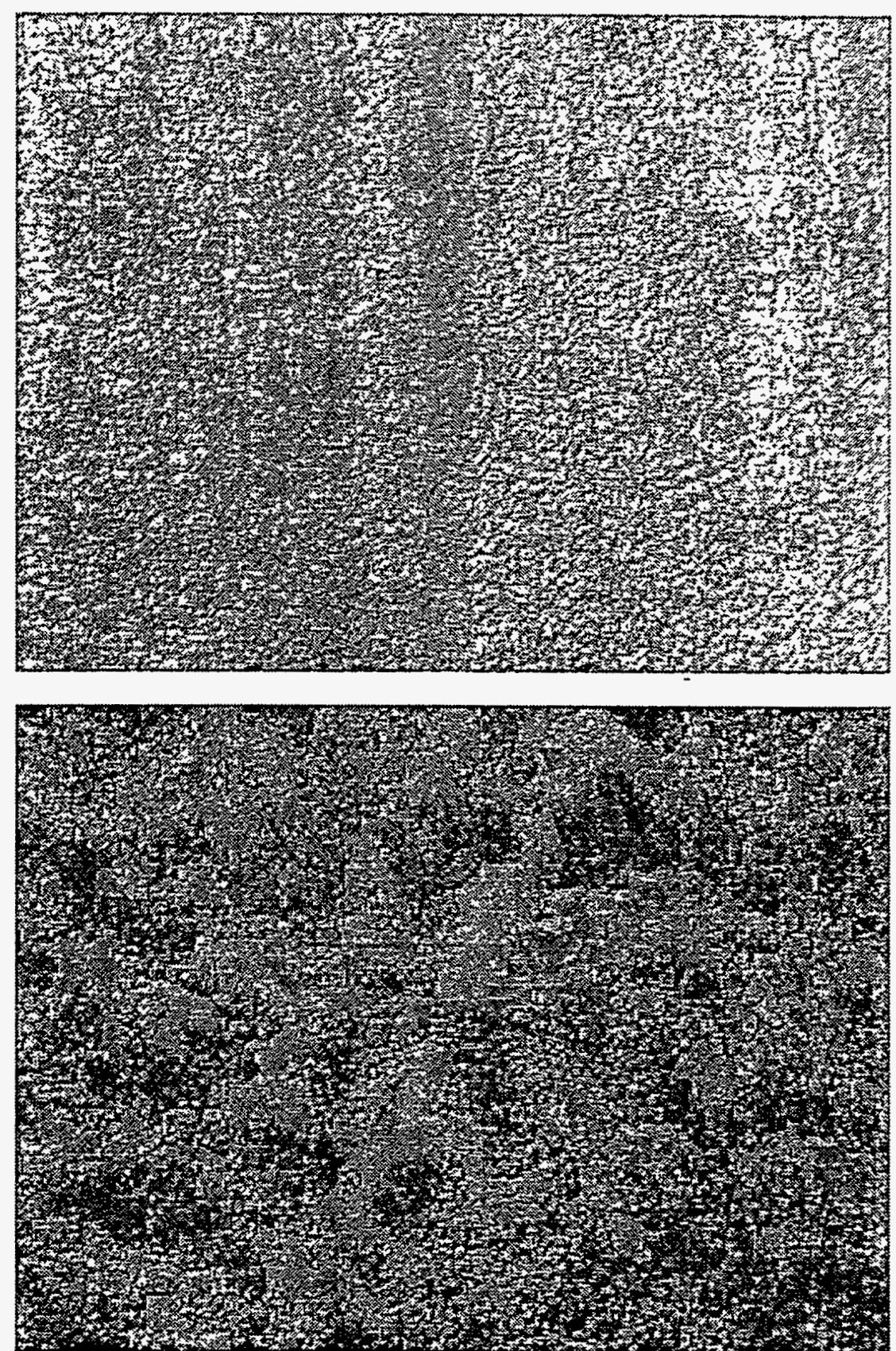

Figure 38(a). Gas-catalyzed film dip coated onto a silicon substrate in the environmental chamber using Preparation D with $r=25 \%$ methanol shows incomplete, spotty coverage, whereas a film made using Preparation F shows continuous, uniform coverage (b).

D.5 Microstructure and Sorptive Properties - Scanning electron microscopy and transmission electron microscopy, Fig. 39, shows that the gas catalyzed films consist of aggregated submicron ellipsoidal or spherical particles. X-ray 
diffraction, Fig. 30, and transmission electron microscopy, Fig. 40, reveal that within each particle there is a periodic hexagonal array of 1-dimensional, unimodal $3 \mathrm{~nm}$ channels. The primary particle size tends to be 20-100 $\mathrm{nm}$ when ammonia gas is used as catalyst and $~ 150-500 \mathrm{~nm}$ when sodium hydroxide is used as a catalyst.

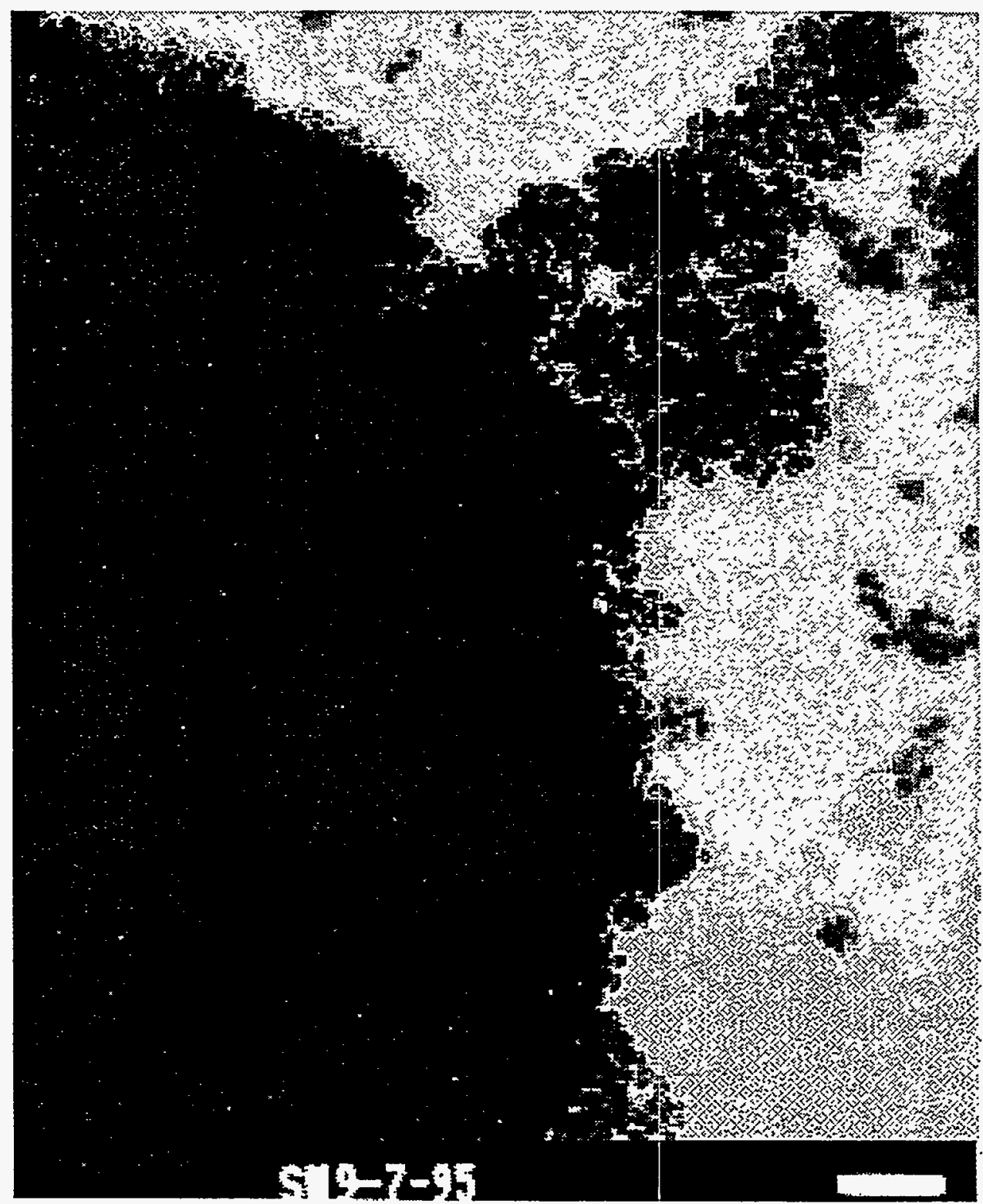

39. Low magnification TEM (scale bar $=500 \mathrm{~nm}$ ) of gas catalyzed film scraped off the substrate shows that the film consists of densely aggregated particles. In this thick film the coverage is complete.

Spin coated gas-catalyzed $r=25 \%$ water:methanol films were coated on ST-cut surface acoustic wave (SAW) devices using method F. The coated 
devices were calcined at $500^{\circ} \mathrm{C}$ in air to remove the organic template from the film. Films less than $1 \mu \mathrm{m}$ did not crack during the calcination procedure. Films greater than $\sim 1 \mu \mathrm{m}$ thick had large cracks after calcination and were not considered further. Nitrogen sorption measurements at $77 \mathrm{~K}$ were collected on a calcined film that was $<1 \mathrm{~mm}$ thick. From BET analysis of the region from $P / P_{0}=0.10$ to 0.18 in the adsorption branch, the surface area of the coated device is 145 times that of the bare device $\left(145 \mathrm{~cm}^{2} / \mathrm{cm}^{2}\right)$. From this value we conservatively estimated the film surface area per gram is $\sim 300 \mathrm{~m}^{2} / \mathrm{g}$ based on a film thickness of $1 \mathrm{~mm}$ and a bulk density of $\sim 0.5 \mathrm{~g} / \mathrm{cc}$. [37]

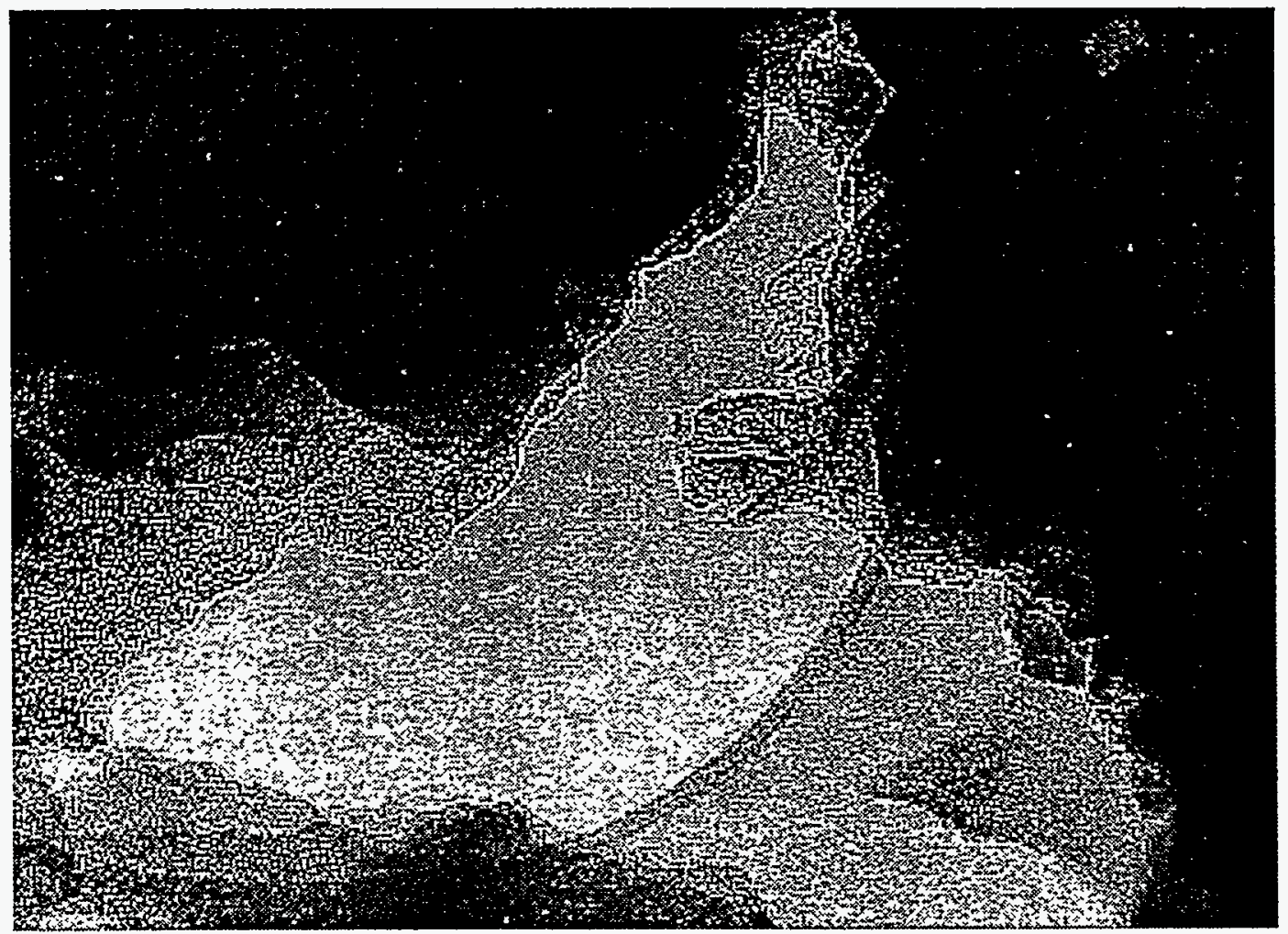

40. Lattice image TEM of gas-catalyzed thin film made from using Preparation $F$ with $r=25 \%$ methanol shows regions of well ordered hexagonal silica. Some areas also appear to have uniform pores that are randomly arranged. This microstructure is expected based on the small CSD size measured in XRD.

\section{E. Conclusions}

We have developed and optimized the synthesis of periodic mesoporous thin films from homogeneous solution. The keys to continuous 
films with complete coverage are: controlling formation kinetics through the use of TMOS as the silica source, the addition of the catalyst in the form of ammonia gas, the use of a controlled atmosphere chamber to control evaporation of the coating solution, and the use of concentrated coating solutions. The films exhibit large surface areas. Films deposited on SAW devices greatly enhance the device sensitivity and provide a size selective porous coating in which the pore diameter can be tuned from 15 to $50 \AA$. The synthetic method described here is flexible and several transition-metal and main-group dopant ions, in the form of alkoxides, have been incorporated into the reactive solution and ultimately the product framework.

\section{Monolithic Periodic Mesoporous Silica Gels}

\section{A. Epitome}

We have synthesized monolithic, templated particulate gels that have pore volumes and surface areas comparable to silica xerogels and aerogels. These gels are unique in that they contain a periodic, tunable microstructure within each particle. The gels exhibit a fascinating microstructure with 5 characteristic length scales over 4 orders of magnitude: fractal domains larger than the particle size ( $>500 \mathrm{~nm}$ ), particles that are 150 to $500 \mathrm{~nm}$ in diameter, interparticle pores that are on the order of the particle size, a feature in the gas adsorption measurements that indicates $\sim 10-50 \mathrm{~nm}$ pores, and periodic hexagonal arrays of $\sim 3 \mathrm{~nm}$ channels within each particle. The microstructure can be controlled by the initial silica content, template size, drying conditions, and calcination conditions. The wet gel monoliths exhibit calculated densities as low as $\sim 0.02 \mathrm{~g} / \mathrm{cc}$; the dried and calcined gels have bulk densities that range from $\sim 0.3-0.5 \mathrm{~g} / \mathrm{cc}$. The materials possess large interparticle $(\sim 1.3-$ $2.3 \mathrm{cc} / \mathrm{g})$ and intraparticle $(\sim 0.3-0.6 \mathrm{cc} / \mathrm{g})$ porosities. 


\section{B. Introduction}

Typical mesoporous materials, such as silica xerogels and aerogels [39], have tailorable microstructures with tremendous pore volumes and surface areas, but the pore structure is irregular and tortuous. For such materials it is possible to assign a characteristic pore size only by using statstical concepts such as percolation [40]. The broad spread of pore sizes is less than optimal for some size- and shape-selective applications, such as chromatography, catalysis, and reaction vessels for the synthesis of quantum confined species. For these applications a mesoporous material with a narrow pore distribution would be advantageous.

It has been recently discovered that by using liquid crystalline surfactant phases as templates enables the synthesis of mesoporous silicas with extremely narrow pore distributions that are tunable from 1.5 to $10 \mathrm{~nm}$ diameter $[1-4,6]$. Reported methods to synthesize periodic mesoporous silica yield precipitates of colloidal particles in a variety of sizes and shapes, ranging from submicron spherical grains to greater than $1 \mathrm{~mm}$ hexagonal aggregates $[1-4,6]$. In effect the pore volume of such aggregates is actually bimodal, one component being the nano-size pores within the particles, the other component being the tortuous avenues through the particle aggregates or compacts, which are of the order of the particle sizes $(\sim 0.150-1000 \mathrm{~nm}$.) Thus the particle size, morphology, and aggregate structure of primary particles are important considerations for applications: these control the flow rate of liquids through the packed material, access to porosity, and the interparticle void volume. These affect the ability to use the material for industrial scale processes (such as pressure swing adsorption), as well as the ability to safely and conveniently handle the material.

We have developed a one-step, binder-free method to synthesize pellets and monolithic periodic mesoporous silica gels (PMSGs) in a wide variety of sizes and shapes. These monoliths exhibit structure on many length scales, consisting of a disordered silica framework (1-10 $\AA$ ), that forms 
nano-size pores $(\sim 30 \AA)$, that are assembled into crystalline domains (4-150 $\mathrm{nm})$, within colloidal particles (20-500 $\mathrm{nm}$ ), that form particle aggregates $(>1$ $\mathrm{mm}$ ), that ultimately form an aggregate gel ( $>1 \mathrm{~mm}$ ). At the higher levels of structure these materials are similar to other aggregate gels (e.g. silica xerogels and aerogels), having the characteristic high surface areas and pore volume. However, each particle is itself porous with volume fraction porosity of $\sim 55 \%$. In this paper we describe the synthesis, microstructure, and sorptive properties of these templated monolithic gels.

\section{Experimental}

C.1 Synthesis -- A typical synthesis of a periodic mesoporous silica gel involves mixing deionized water, methanol, cetyltrimethylammonium bromide (CTÁB), NaOH, and tetramethoxysilane (TMOS). The water to methanol ratio is fixed at $75: 25 \mathrm{w} / \mathrm{w}$ and the micellar solutions, before the addition of TMOS, are $1,2,4,8,16,32 \mathrm{wt} \%$ in CTAB solutions. After addition of the TMOS, the silica concentrations are $0.2,0.4,0.76,1.38,2.33,3.56 \mathrm{M}$, respectively. The $\mathrm{H}_{2} \mathrm{O}$ :Si ratios vary from 195 to 4.2 across this range. The CTAB:NaOH:TMOS ratio is kept constant at 0.13:0.27:1.

The porous materials were synthesized by injecting TMOS into a rapidly stirred, basic surfactant solution. The resulting monolithic gels were dried in air over several days and then calcined to remove the surfactant. Calcination involved heating from 25 to $550^{\circ} \mathrm{C}$ at $1{ }^{\circ} \mathrm{C} / \mathrm{min}$ in flowing $\mathrm{N}_{2}$ followed by a $1 \mathrm{~h}$ isotherm and a $1^{\circ} \mathrm{C} / \mathrm{min}$ cool to room temperature; the process is repeated in flowing air except the isotherm lasted $5 \mathrm{~h}$. This procedure avoids the pore collapse that can occur if the monoliths are calcined in air. The calcined products are white.

C.2 X-ray Diffraction -- Data were collected on crushed monoliths using a Scintag PAD V instrument equipped with nickel-filtered $\mathrm{Cu} \mathrm{K}_{\mathrm{a}}$ radiation. A continuous scan mode was used to collect data from 1.5 to $10^{\circ} 2 \theta$ with a $0.02^{\circ}$ 
sampling interval and a $1 \% \mathrm{~min}$ scan rate. Slits widths starting from the source were $1,2,1$, and $0.3 \mathrm{~mm}$. Tube voltage was $45 \mathrm{kV}$ and tube current was $35 \mathrm{~mA}$.

C.3 Small Angle X-ray Scattering (SAXS) -- Data were collected on a BonseHart camera at Brookhaven National Light Source. This SAXS instrument uses highly collimated $X$-rays available on beam line $X 8 \mathrm{C}$ at the National Synchrotron Light Source. The analyzer used is a channel cut Si(111) crystal specifically designed for use at $\lambda=1.54 \AA$; it has a measured efficiency of $60 \%$ and an angular resolution (FWHH) of $0.001^{\circ}$. In the case of strongly scattering samples (i.e. powders), momentum transfers in the range $1 \times 10^{-4} \AA^{-1}<\mathrm{q}<0.1$ $\AA^{-1}$ could be studied with over 8 decades of dynamic intensity range. The analyzer crystal was rocked about its Bragg position to filter out only those $X$ rays with a given scattering angle. A scintillation counter was utilized to obtain a point-by-point scattering curve from a given sample. A major benefit of this camera is that the sample to detector distance of the $X$-ray camera is not determined by the desired angular resolution, and therefore is small even for high resolution work. For example, in pinhole cameras typical sample to detector distances are $10 \mathrm{~m}$, and even so they often have over an order of magnitude less resolution than a compact Bonse-Hart design.

C. $4{ }^{29} \mathrm{Si} N M R$ - High resolution, magic-angle-spinning spectra were recorded on a Chemagnetics instrument equipped with a 4.7 Tesla magnet. Powder samples were packed into $2.5 \times 6 \mathrm{~cm} \mathrm{ZrO}_{2}$ rotors. Spectra were recorded at a resonance frequency of $39.7 \mathrm{MHz}$, with a $7.25 \mathrm{~ms}$ pulse at $90^{\circ}$, and a pulse delay of $120 \mathrm{~s}$. Final spectra are an average of 512 scans. Tetramethylsilane (TMS) was used as a standard to define $0 \mathrm{ppm}$. Data were analyzed and integrated with MacNMR software routines from Tecmag to determine $Q$ ratios and shifts relative to TMS. 
C.5 SEM/TEM - A JOEL 1200EX transmission electron microscope (TEM) with ASID (SEM) attachment was used to observe the microstructure and grain size of the crushed monoliths. In order to observe individual grains, the aggregated powders were ground under methanol in a mortar and pestle. The suspended powder was caught on the holey carbon film of a $3 \mathrm{~mm}$ copper grid. Bright field TEM or diffraction contrast imaging was done at $120 \mathrm{kV}$ and involved low (20k times) and high (300k times) magnifications of the individual grains and small aggregates. SEM micrographs were obtained that reveled the morphology of powder aggregates and the size of the grains.

C.6 Gas Adsorption - A Micromeritics ASAP 2010 was used to collect isothermal $\mathrm{N}_{2}$ adsorption data at $77 \mathrm{~K}$ on crushed and monolithic samples. Samples were degased overnight at $200{ }^{\circ} \mathrm{C}$. 4-point BET analyses were performed to determine the apparent surface areas. Pore diameters were determined by application of the Kelvin Equation to the desorption branch of the data.

\section{Results and Discussion}

D.1 Silica Source: The Key to Forming Monolithic PMSGs - Typical mesoporous silica syntheses produce colloidal precipitates after 10-15 minutes. Our discovery of periodic mesoporous silica gels (PMSG) resulted from an attempt to optimize solution homogeneity, kinetics, and product yield for bulk periodic mesoporous silica powders [37]. These gels form in seconds at room temperature. We examined several silica sources and solvent systems [38] (e.g. water plus alcohols, amides, glycols, acetates, or polyethers) and found that the use of non-molecular solid silica sources, such as, fumed silica, precipitated silica, silica sols, or water glass, or the use of molecular alkoxides, such as, tetraethoxysilane (TEOS), tetrapropoxysilane (TPOS), tetrabutoxysilane (TBOS), results in colloidal particles of PMS in less 


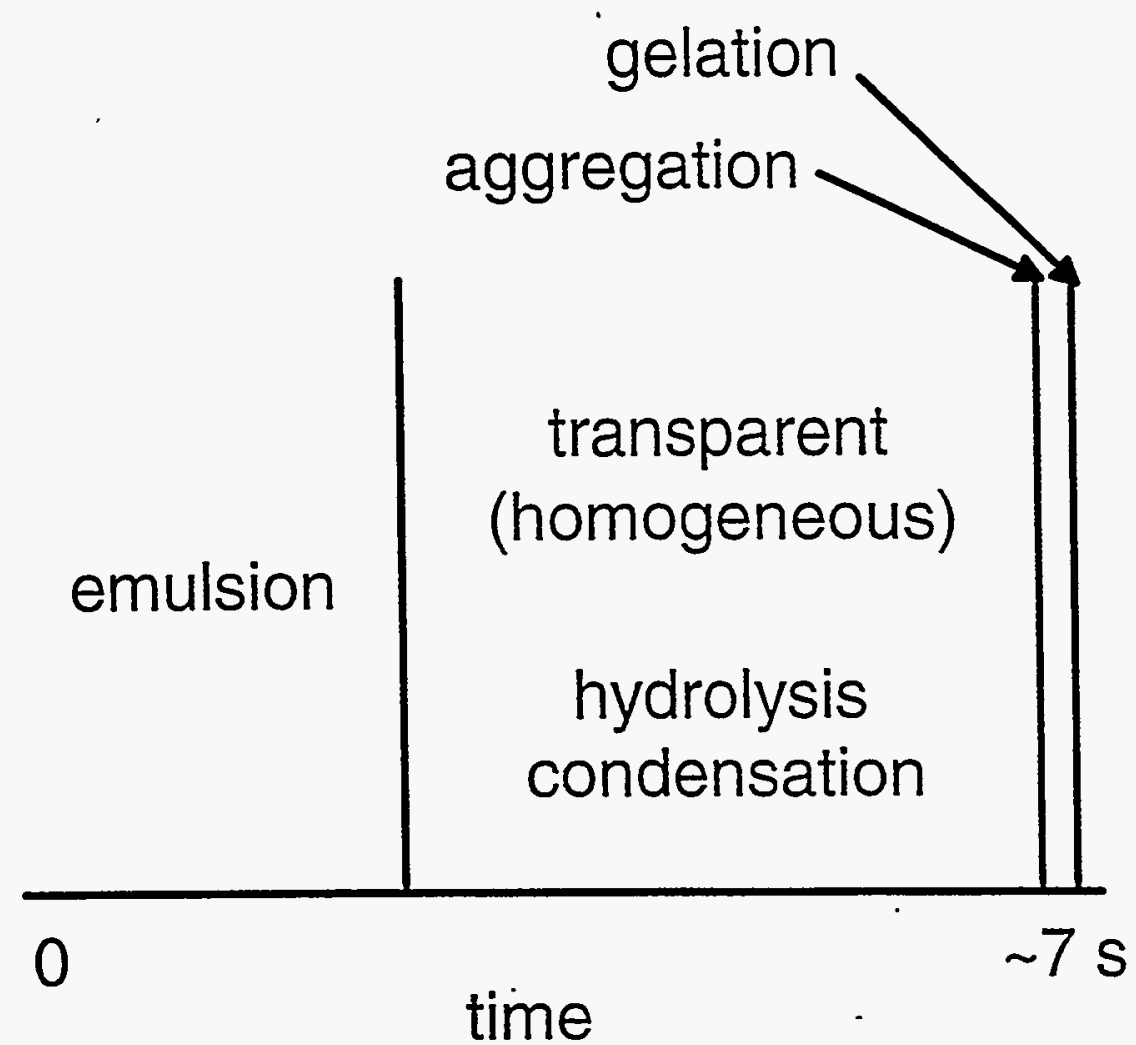

Figure 41. Evolution of product formation at room temperature for a sample that contains $2 \mathrm{wt} \%$ $\mathrm{CTAB}$ (in the initial solution), $[\mathrm{Si}]=0.40 \mathrm{M}$, and $[\mathrm{NaOH}]=0.174 \mathrm{M}$ in a $75: 25$ water:methanol solution. The time axis corresponds to immediately after the TMOS is added to the alkaline micellar solution.

than optimal yield $[1-4,6]$. In addition, the kinetics of these reactions are sluggish so that at any given time there are a wide variety of species in solution.

To increase the reaction kinetics, we used tetramethoxysilane (TMOS) as the silica source, which is known to hydrolyze quickly. To insure solution homogeneity, improve yield, and optimize long-range order, we used the cosolvent methanol [37]. From these homogeneous solutions, monolithic gels of periodic mesoporous silica form within 5-7 seconds at room temperature. The evolution of product formation is depicted in Fig. 41: TMOS is added to the alkaline micellar water:methanol solution $(t=0)$, an emulsion forms initially, but as the TMOS partially hydrolyzes it dissolves (within $\sim 3$ seconds) to yield a clear transparent solution. This solution turns translucent within 1-3 seconds as particles form, and rapidly becomes opaque as these particles aggregate and the aggregates gel. Gelation occurs with $\sim 1 \mathrm{~s}$ 


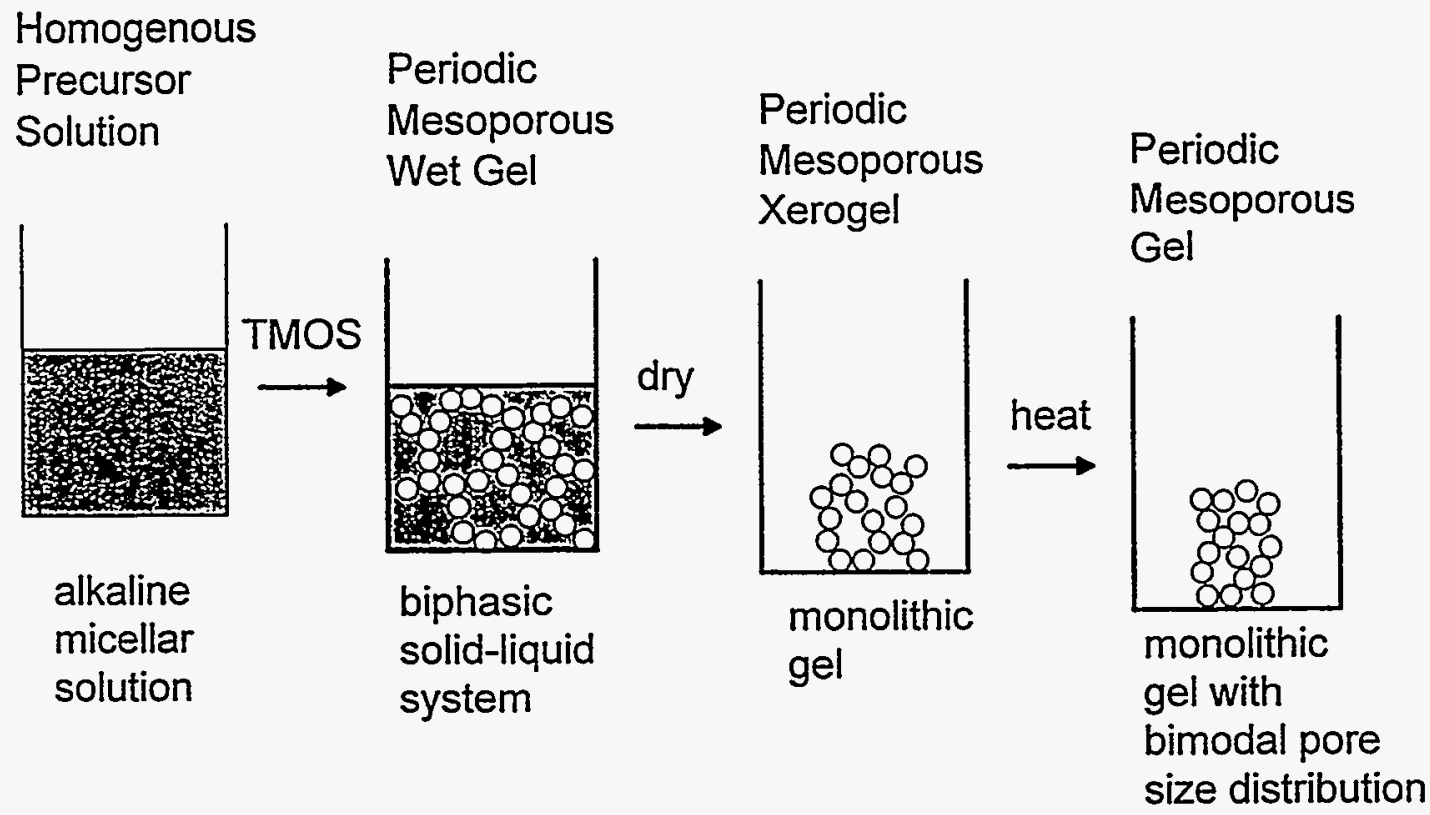

Figure 42. Schematic of product synthesis and processing.

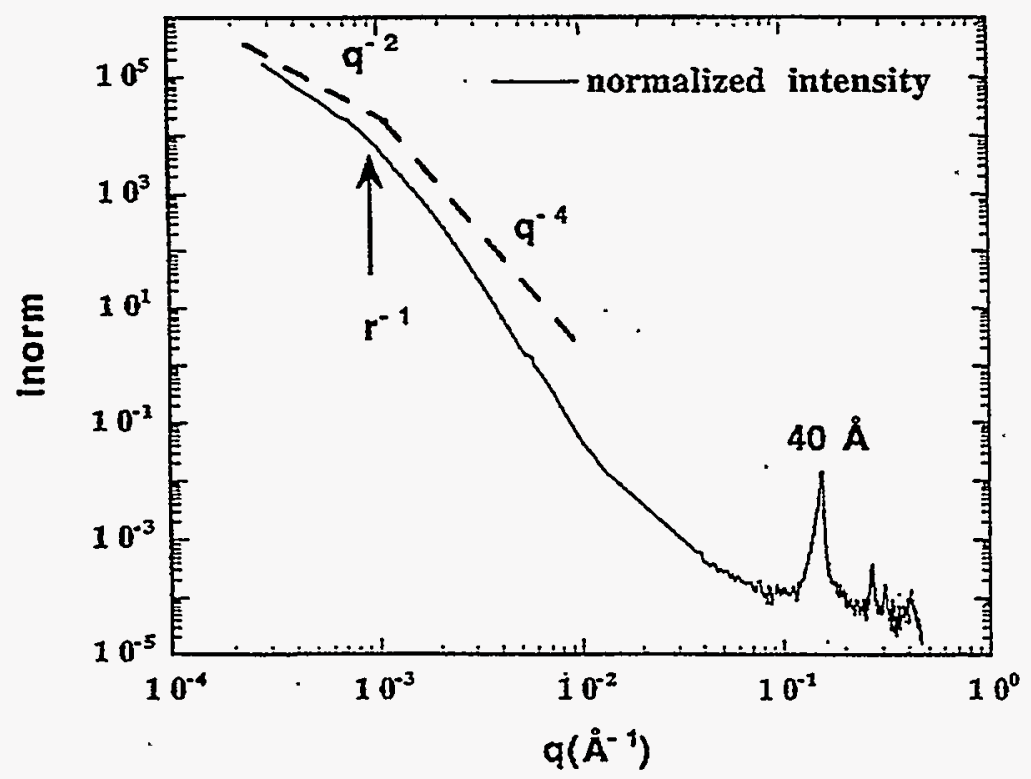

Figure 43. SAXS data show an extended regime of power-law scattering, indicative of aggregation of particles (mesophase domains) into fractal clusters of dimension 2. The slope of 4 in the Porod regime indicates the existence of smooth particles. The diffraction peaks at large $\mathrm{q}$ indicate the existence of a hexagonal array of unimodal $\sim 4 \mathrm{~nm}$ pores. 
after the onset of visible turbidity. An overview of the subsequent synthesis and processing steps is shown in Fig. 42.

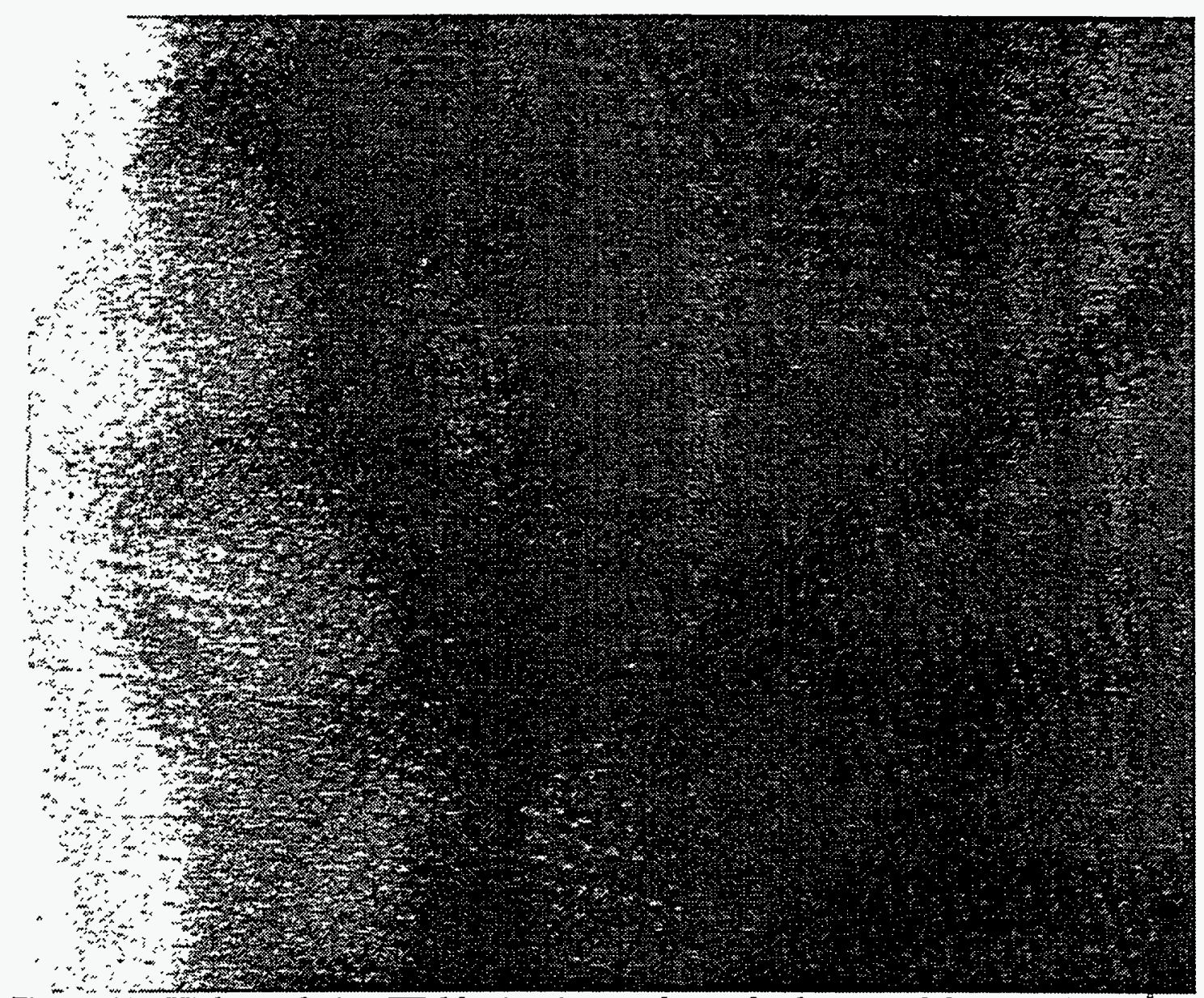

Figure 44. High resolution TEM lattice image shows the hexagonal lattice of $\sim 30 \AA$ pores within a particle.

D.2 Structure of PMSGs - We have examined the structure of the gels on several length scales with high resolution small angle $x$-ray scattering (SAXS) measurements and scanning and electron microscopies. The SAXS data are shown in Fig. 43. In the Bragg region of the SAXS data we see 4 peaks that can be indexed as the 100,110,200, and 210 reflections of a hexagonal cell ( $a$ »46 $\AA)$. This is definitive evidence for a periodic mesoporous silica 1-d channel structure, and data in this scattering regime agree well with our more conventional diffractometer measurements discussed below. High resolution TEM of the particles, Fig. 44, also confirms the existence of periodic hexagonal arrays of 3-4 $\mathrm{nm}$ pores. 
The coherent scattering domain size (CSD) of the periodic arrays can be computed from the width of the Bragg reflections using the Scherrer equation. This analysis gives CSDs in the range of 4-150 $\mathrm{nm}$, depending on the synthesis conditions, especially on the cosolvent type and concentration.

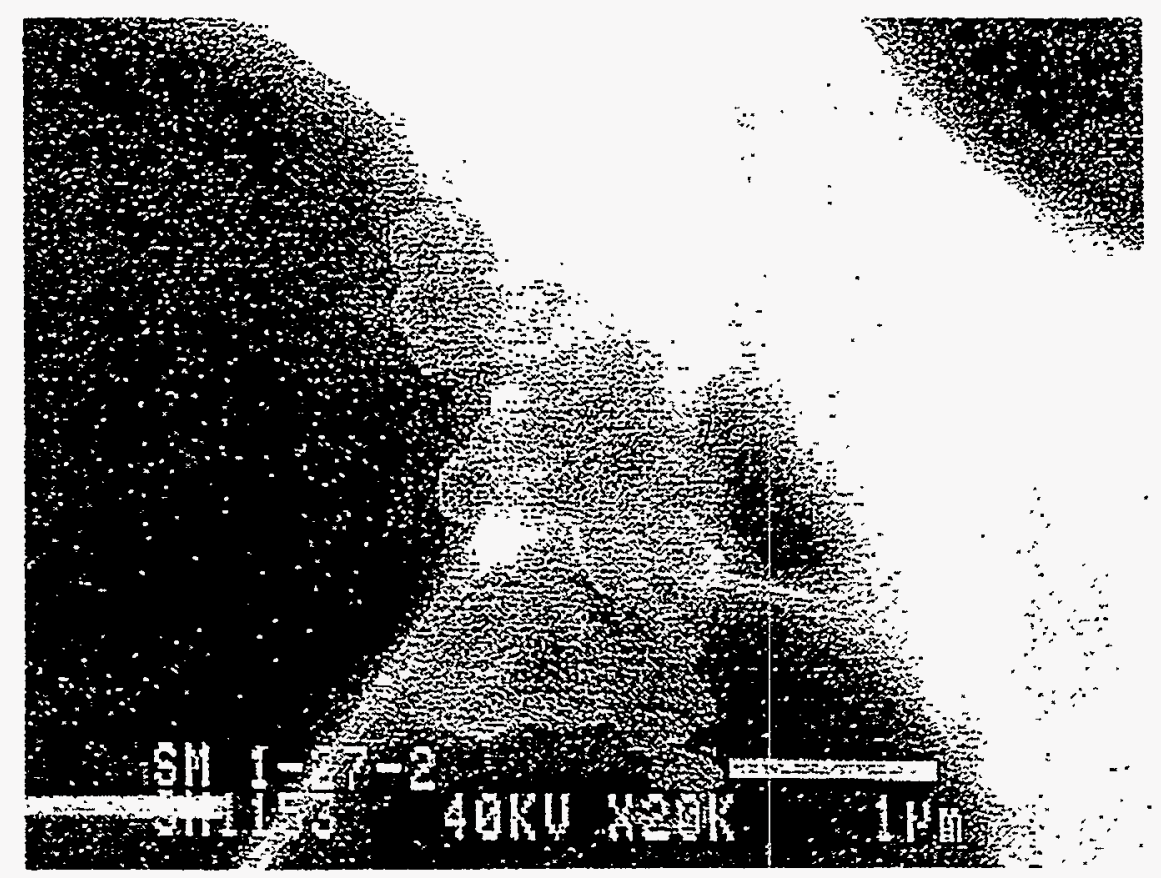

Figure 45. Scanning electron microscopy shows the aggregated $\sim 150-500$ mm ellipsoidal particles obtained from the crushed gel.

In the so-called Porod region $\left(a<<q^{-1}<R\right)$ of the SAXS data, where the scattering is due to the discontinuity in electron density at the particle surface, we observe a slope of approximately -4 on $\log -\log$ axes, $\left(S(q) \sim q^{-4}\right)$ which is indicative of smooth colloidal particles. The particles are spherical or ellipsoidal and range in size from $\sim 150$ to $\sim 500 \mathrm{~nm}$, as shown in Fig. 45, which may account for some of the deviation from Porod's law.

For dimensions large compared to the particle size $\left(\mathrm{q}<8 \times 10^{-4} \AA^{-1} ; \mathrm{d}>\right.$ $785 \mathrm{~nm}$ ), we see evidence of particle aggregates. Colloidal aggregates are very often mass fractal structures which give the scattering behavior $S(q) \sim q^{-D}$, where $1<\mathrm{D}<3$ is the fractal dimension. The fractal dimension, as determined 
from the slope of this data on $\log -\log$ axes, is $\sim 2$. (Additional evidence for the fractal nature of these aggregates is the formation of a gel, as discussed below.)

Finally, the aspect of growth that is technically due to gelation physics cannot be observed from scattering data taken on undiluted gelling structures. Gelation gives rise to connectivity correlations, not spatial correlations (although under very special circumstances connectivity correlations can induce a phase transition that itself produces spatial correlations) and scattering is only sensitive to the latter.

D.3 Aggregation and Gelation - Because mesoporous silica gels have not been studied previously, we will now describe the aggregation mechanism and its effects on mechanical properties in more detail. As mentioned above, on length scales large compared to the particle size, the SAXS data in Fig. 43 show the power law scattering $I(q) \sim q^{-D}$, with $D \cong 2$, which is consistent with the formation of fractal particle aggregates.

A reasonable scenario for aggregation is as follows: (1) TMOS hydrolyzes to form multidentate, multiply charged silicate oligomers that displace the monodentate, monovalent surfactant counterions (Br) from the surfactant micelles; (2) because these micelles now have an attractive interaction, due to bridging by multidentate ligands, [41] the micelles will stick upon contact, nucleating silica/surfactant rich domains; (3) these domains quickly grow due to further collisions with micelles and/or through coalescence with other domains; (4) the silica/surfactant rich domains undergo a transition to a liquid cystalline phase to form the mesoporous particles; (5) the particles collide to form aggregates, probably held together by siloxane bonds at interparticle contacts; (6) the aggregates grow to fill the volume of the solution and then percolate to form a gel; (7) the solid component of the gel continues to condense, thus consolidating the silica framework. Of course, other mechanisms for the formation of the particles have been suggested, such as the formation of silica-encapsulated rod-like 


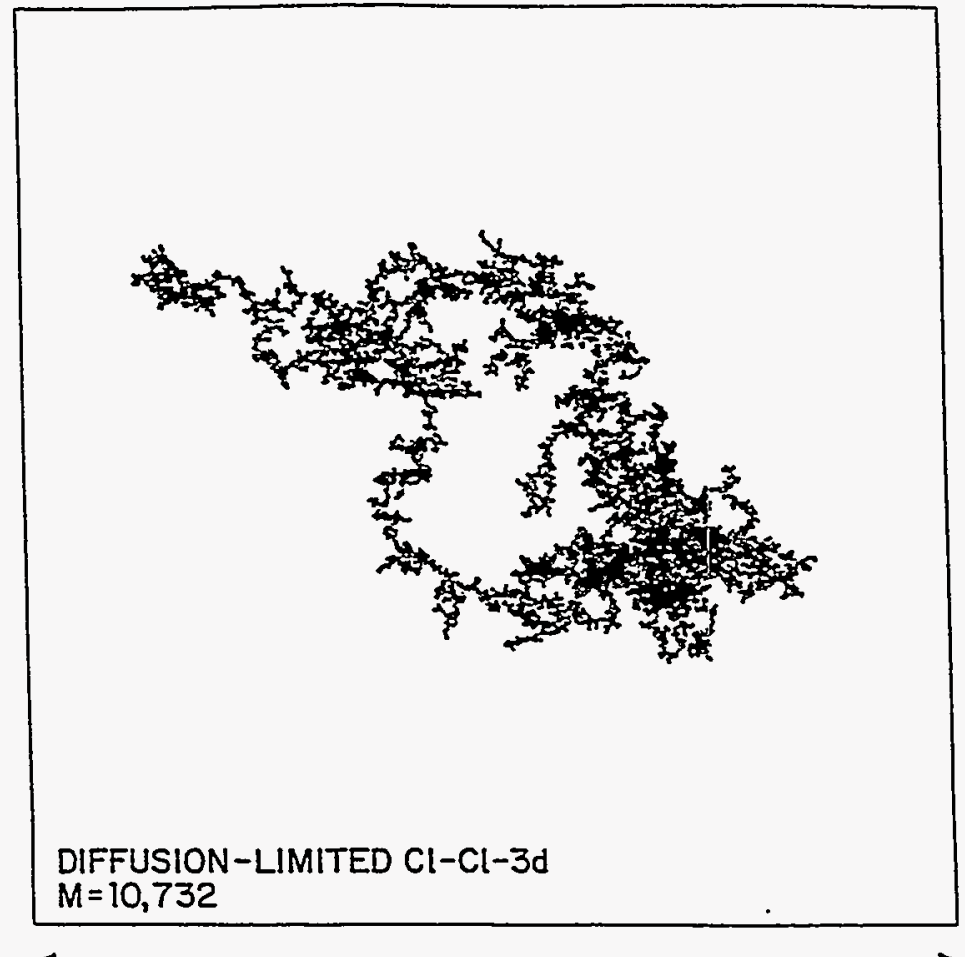

260 DIAMETERS

Figure 46. A 3-d Diffusion-Limited Cluster-Cluster Aggregate as simulated by P. Meakin [45]. Simulations give a fractal dimension of $\sim 1.75$.

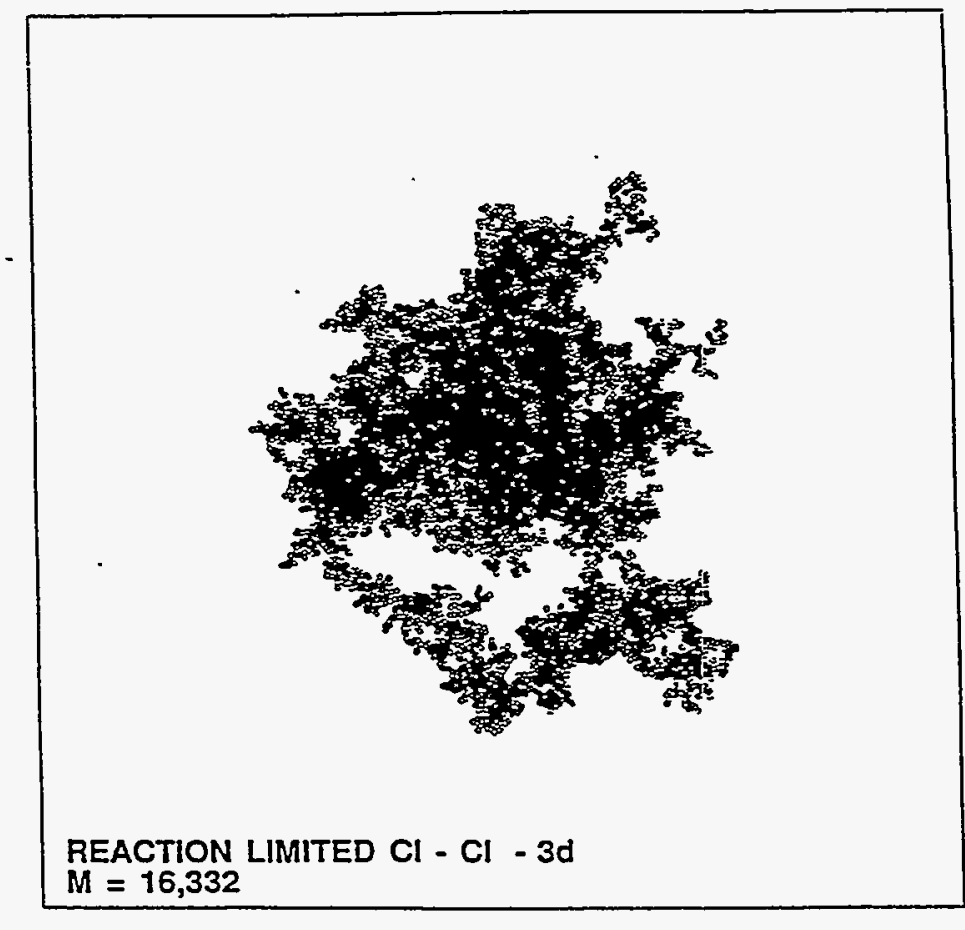

180 DIAMETERS

Figure 47. A 3-d Reaction-Limited Cluster-Cluster Aggregate as simulated by P. Meakin [45]. Simulations give a fractal dimension of $\sim 2.05$. 


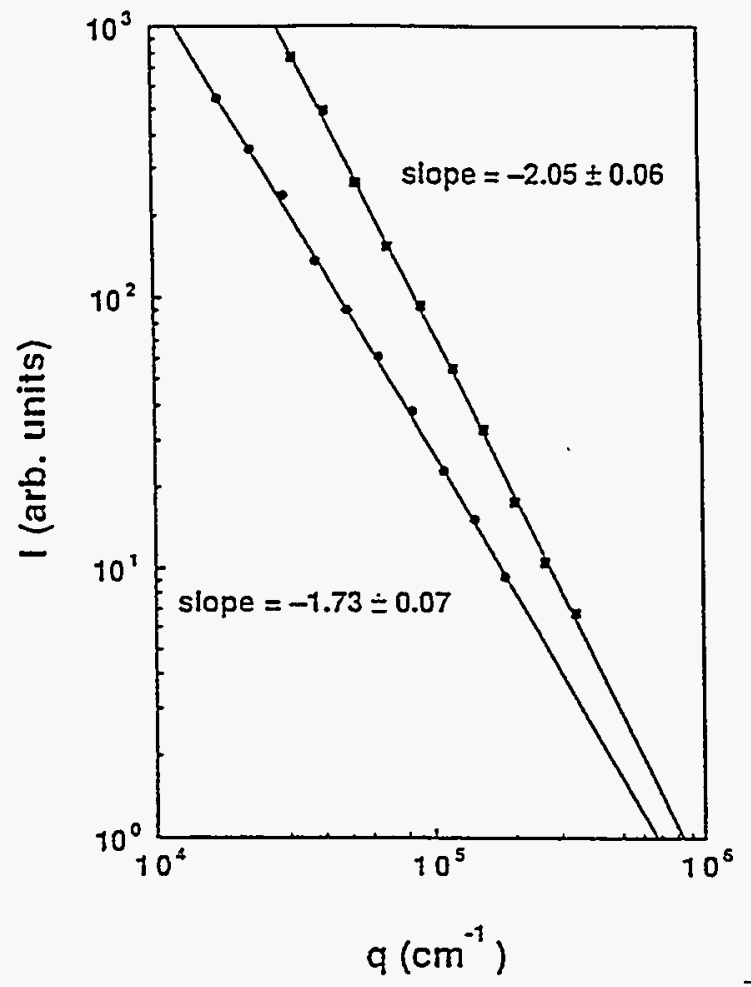

Figure 48. Light scattering data for rapid aggregates formed at a concentration of $2.5 \times 10^{-4} \mathrm{wt} \%$ silica aqueous solution give a fractal dimension of $1.73 \pm 0.07$, in constant with the data for slowly formed aggregates at a concentration at $0.1 \mathrm{wt} \%$ silica, which gives $\mathrm{D}=2.05 \pm 0.06$ [45].

micelles that then assemble to form bundles, and we cannot rule these out, so this description is only based on what we believe is reasonable. The timescale of particle formation is exceedingly small, so it will be difficult to sort this out. We will thus discuss the latter stages of growth.

Fractal aggregation has been much studied in the last decade [35], and it is found that it generally falls into two classes: Diffusion-Limited Cluster Aggregation (DLCA), Fig. 46, in which the clusters stick upon first contact, and Reaction-Limited Cluster Aggregation (RLCA), in which the sticking probability of the clusters is less than unity, Fig. 47. DLCA produces clusters with a fractal dimension of -1.75 , and RLCA produces clusters with a fractal dimension of $\sim 2.1$, Fig. 48 . Note that these data are taken on silica without surfactant. We expect surfactant to have two effects: it templates the periodic structure and reduces the repulsive Coulombic interaction between particles by reducing the charge. Thus the surfactant catalyzes gel formation by enabling the rapid formation of particles, and the susequent rapid aggregation 


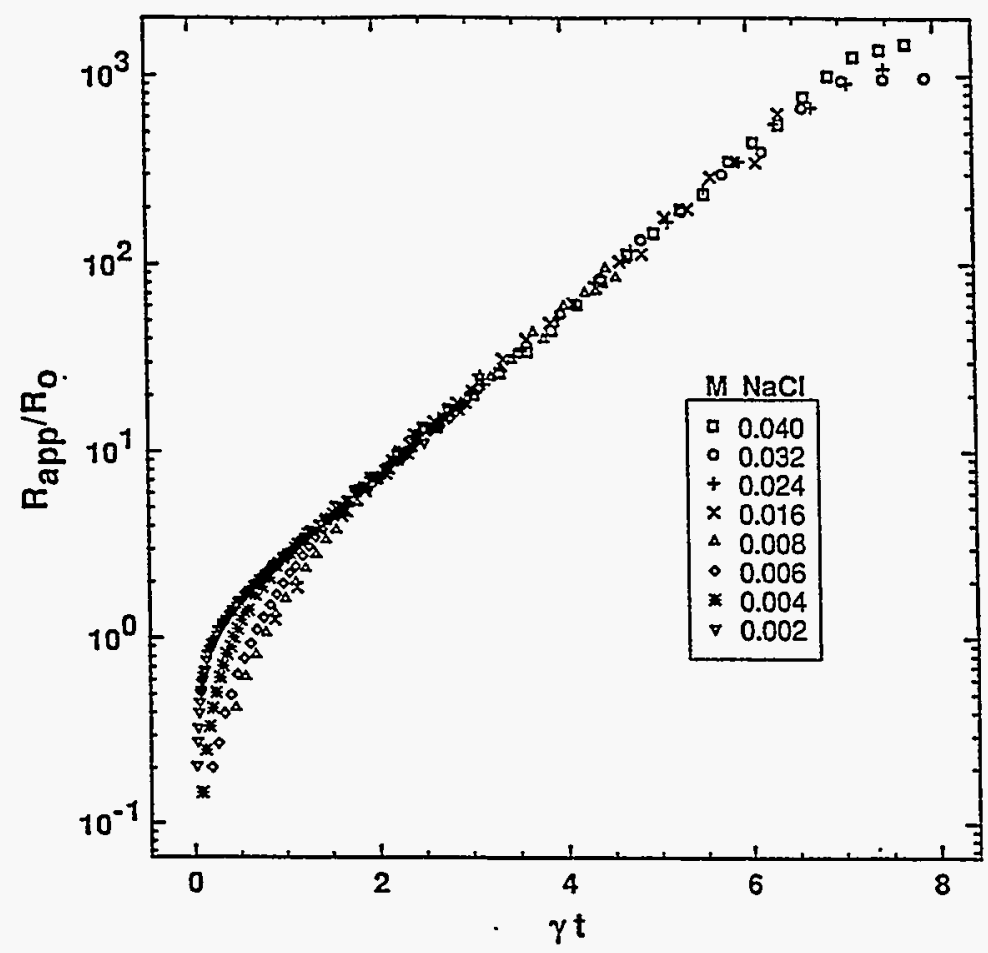

Figure 49. Reaction-Limited Cluster-Cluster Aggregation is exemplified by light scattering data from methanol solutions that were $100 \mathrm{mM}$ TMOS, $4.4 \mathrm{mM} \mathrm{NH}_{4} \mathrm{OH}$, and $2.2 \mathrm{M} \mathrm{H}_{2} \mathrm{O}$ [46]. The data show a collapse to a universal exponential growth regime for gt $>1$, which indicates RLCA.

of these. We have conducted a control experiment without the surfactant and a gel forms not in seconds, but in $\sim 10 \mathrm{~h}$.

Based on the extremely rapid aggregation and the high concentration of reactive hydroxyl and alkoxy species at the gel point (see below), we expect DLCA to be the operative growth mechanism; however, the measured fractal dimension is more consistent with RCLA. Generally, growth kinetics measurements can resolve these ambiguities: DLCA gives power law growth of the cluster radius, $R$, Fig. 49, and RLCA gives an exponential increase, Fig. 50. Unfortunately, in the case of periodic mesoporous silica, the aggregation is too rapid to study growth kinetics.

We attempted to slow the growth by cooling the samples as well as by limiting the amount of base catalyst added. Cooling the samples to $-14{ }^{\circ} \mathrm{C}$ slows the gel formation time to $\sim 90 \mathrm{~s}$, but the aggregation phase occurs in less than the last $5 \mathrm{~s}$, which is far too rapid for time-resolved static or dynamic 


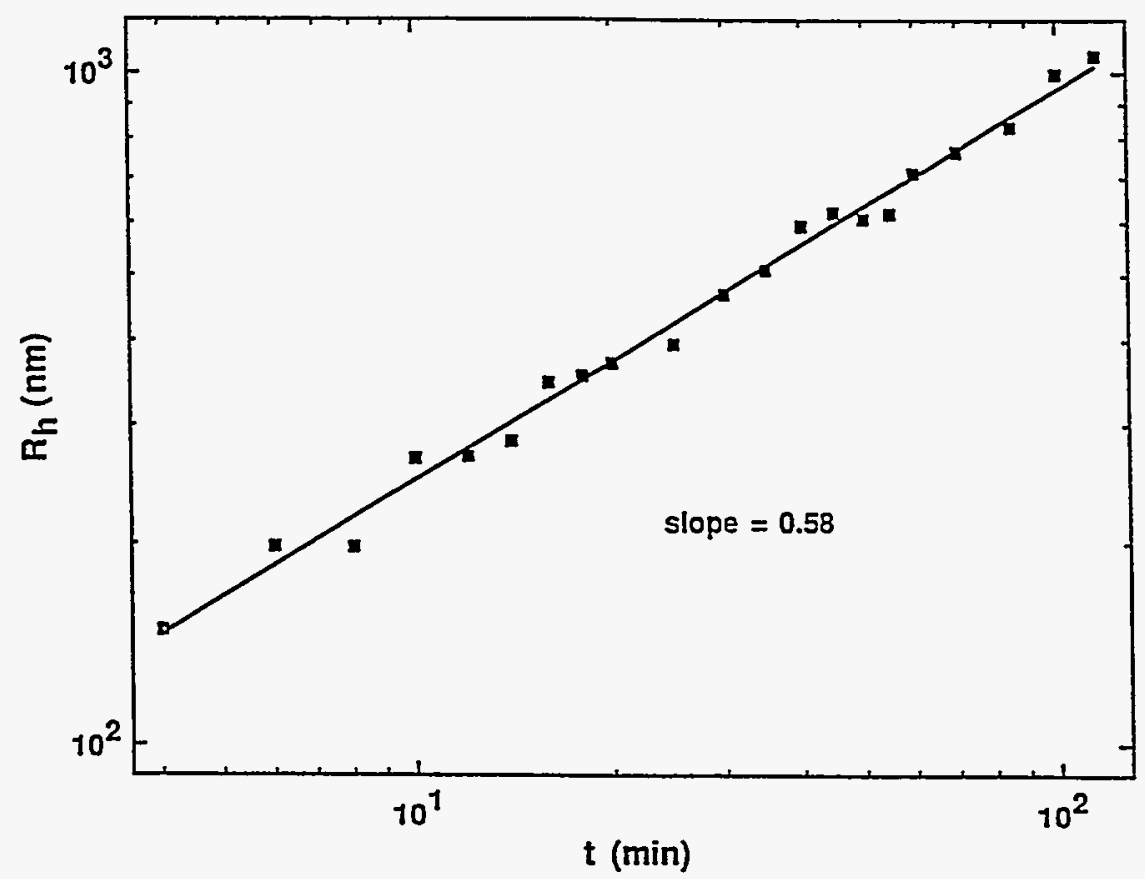

Figure 50. Rapid Diffusion-Limited Cluster Aggregation is exemplified by light scattering data from a methanol solution that is $1000 \mathrm{mM} \mathrm{NaCl}, 30.0 \mathrm{M} \mathrm{H}_{2} \mathrm{O}, 40 \mathrm{mM} \mathrm{NH}_{4} \mathrm{OH}$, and $1 \mathrm{mM}$ TMOS [46]. The power-law growth is indicative of DLCA.

light scattering measurements. Reducing the amount of base in the system has little effect on gelation time until the $\mathrm{pH}$ is less than $\sim 9$. But from $7<\mathrm{pH}$ $<9$ only amorphous silica chemical gels form.

Regardless of the growth mechanism, there are universal aspects of aggregation that provide insight into the structure and properties of a gel. For example, suppose that just after the mesoporous particles form they occupy a volume fraction $\phi_{p}=(4 p / 3) n a^{3} / V$, where $n$ is the number of particles, $a$ is the particle radius, and $V$ is the solution volume (in our case $\phi_{p} \gg 0.018$ to 0.099 for $[\mathrm{Si}]=0.40$ to $2.33 \mathrm{M}$ ). As these particles aggregate to form clusters of overlap radius $R$ (the radius of cluster-cluster contact) the solution volume they occupy increases with radius as $\phi_{c l}=(4 \pi / 3) N R^{3} / V$, where $N \sim(a / R)^{D} n$ is the number of clusters. Note that $\phi_{c l} \sim R^{3-D}$, so for mass fractal clusters, with $D<3$, the solution will become more congested as the clusters grow, until the 
clusters reach the point where they occupy the solution volume, $\phi_{c l}=1$. At this point of overlap the radius will be $R^{*} \sim a \phi_{p}^{-1 /(3-D)}$.

At the point of overlap the growth is no longer limited by cluster diffusion. Rather, it becomes limited by the condensation rate, or by fluctuations in the aggregate structure (bending motions of arms etc.). The densely packed clusters will then randomly bond to neighbors to form a gel network. We expect this gelation to be described by the percolation universality class, as demonstrated by simulations and experiments [42], and the simple fact that other gelation universality classes have not been discovered!

The final gel will be homogeneous on length scales larger than the socalled spatial correlation length $\xi$. In other words, on length scales larger than $\xi$ the pair correlation function $\rho(r)$ will be a simple constant. On length scales smaller than $x$ the pair correlation function will scale like $\rho(r) \sim 1 / r^{3-D}$. For these aggregate gels the spatial correlation length will be the overlap radius $R^{*}$ (ca. $7.0 \mathrm{~mm}$ to $1.3 \mathrm{~mm}$ for [Si] $=0.40$ to $2.33 \mathrm{M}$ and $\mathrm{a}_{\mathrm{ave}}=125 \mathrm{~nm}$ ). Note that the overlap radius will increase with an increase in the dimension $D$. This overlap radius will also increase with decreasing particle concentration, and one can argue that the final shear modulus [43] of the gel will be proportional to $1 / \xi^{3} \sim 1 /\left(a \phi_{p}^{-1 /(3-D)}\right)^{3} \sim \phi_{p}^{3}$.

D.4 Controlling the Interparticle Porosity - The interparticle porosity in the gel monolith is primarily determined by the volume fraction of particles $\left(\phi_{\mathrm{p}}\right)$, which is determined by the initial concentration of silica in the precursor solution. For many applications it is desireable to have a large interparticle pore volume (i.e. low particle fraction) and yet it is not clear whether mesoporous particles will form at very low silica/surfactant concentrations. Thus we investigated the range of silica concentrations for which periodic products form. 


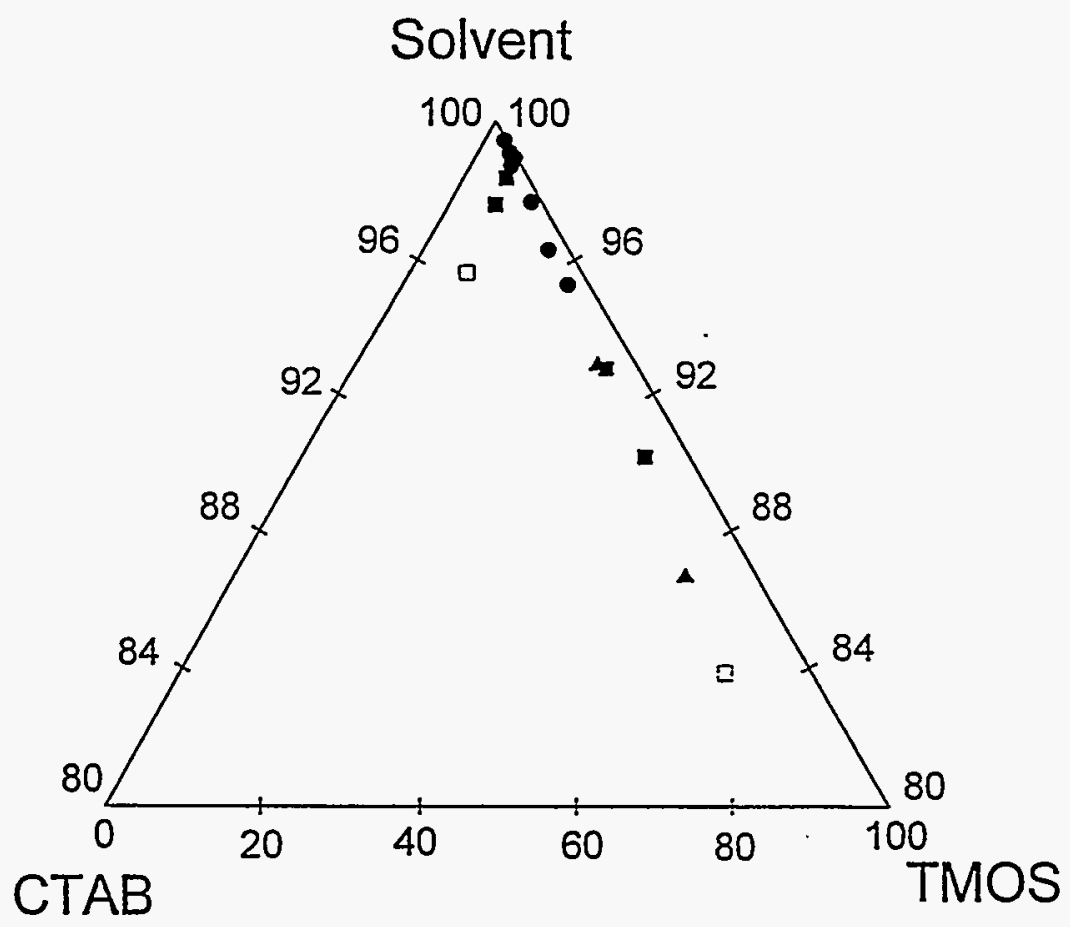

Figure 51. Phase diagram shows the formation of products with 4 or more low angle diffraction peaks (circles), three diffraction peaks (filled squares), 1 diffraction peak (triangle), and mo low angle diffraction peaks (open square). The TMOS: $\mathrm{NaOH}$ ratio was kept constant at 1:0.27 and the solvent was 75:25 water:methanol.

We first examined the solvent-rich portion of the water:methanol:TMOS:CTAB phase diagram with $r$ fixed at 25\%, as shown in Fig. 51. Here $r$ is the weight \% methanol in the cosolvent mixture. We concentrated on the line that provides optimal periodicity in the $[\mathrm{Si}]=0.40 \mathrm{M}$ preparations, that is, CTAB:TMOS molar ratio of $\sim 0.13$. The phase diagram shows that well ordered products form over a large range of water:silicon ratio, extending to low particle concentrations. In fact, periodic products can be made from solutions range from $\sim 0.1 \mathrm{M}$ to $\sim 6.0 \mathrm{M}$ in TMOS. These correspond to a $0.5 \mathrm{wt} \%$ to $32 \mathrm{wt} \% \mathrm{CTAB}$ solution, which, for a $75: 25$ water:methanol, corresponds to a $\mathrm{H}_{2} \mathrm{O}: \mathrm{Si}$ ratio of $\sim 390$ to -3.9 .

Having shown that we can indeed make the mesoporous particles at low silica concentration, the next issue is the mechanical stability of such low density gels, especially as regards their drying and shrinkage. To this end, we synthesized a series of monolithic gels that contained $[\mathrm{Si}]=0.20,0.40,0.76$, 1.38, 2.33, and $3.56 \mathrm{M}$, Si:CTAB:OH = 1:0.13:0.27 with a $75: 25 \mathrm{w} / \mathrm{w}$ 


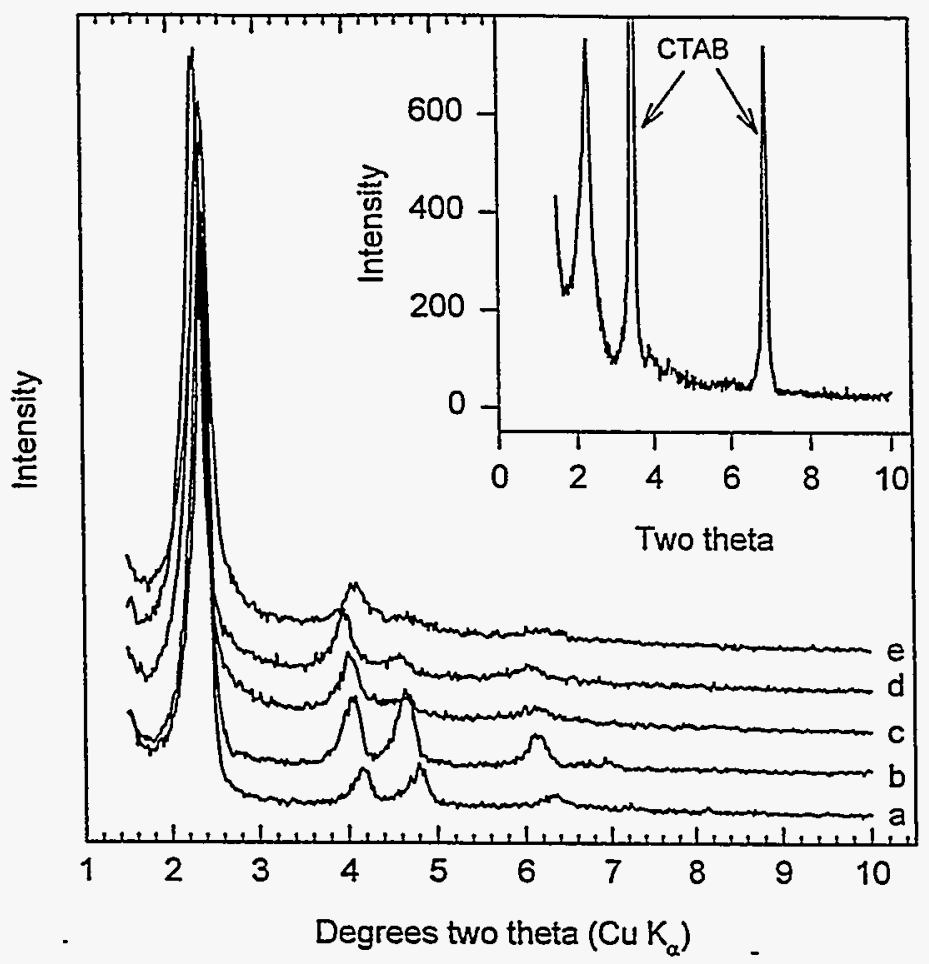

Figure 52. X-ray diffraction data recorded on samples made from $[\mathrm{Si}]=0.20 \mathrm{M}(\mathrm{a}), 0.40 \mathrm{M}(\mathrm{b})$, $0.76 \mathrm{M}$ (c), $1.38 \mathrm{M}$ (d), $2.33 \mathrm{M}$ (e), and $3.56 \mathrm{M}$ (inset) solutions with CTAB wt\% =1, 2, 4, 8, 16, and $32 \mathrm{wt} \%$ respectively; $\mathrm{Si}: \mathrm{NaOH}=1: 0.27$; and 75:25 water:methanol. Note the optimal long range order occurs for the [Si] $0.40 \mathrm{M}$ sample as indicated by the 4 sharp diffraction peaks. The $[\mathrm{Si}]=3.56 \mathrm{M}$ sample contains much unreacted CTAB.

water:methanol ratio. The diffraction patterns, Fig. 52, show that all these preparations lead to periodic mesoporous silica gels, but in the last case, even after washing, there was a high concentration of unreacted CTAB and thus a relatively low yield of PMS.

The particle density of the wet gel monoliths (as calculated by dividing the weight of added silica plus surfactant by the observed gel volume) can be quite low. For example, with [Si] $=0.40 \mathrm{M}$ the particle density is $\sim 0.02 \mathrm{~g} / \mathrm{cc}$ and with [Si] $=3.56$ the density is $0.20 \mathrm{~g} / \mathrm{cc}$, Fig. 53 . However, the density of the dried monoliths can be much larger, depending on the shrinkage experienced during drying. For PMSGs the total monolith volume shrinks $\sim 90 \%$ to $\sim 60 \%$ when the wet gels are dried in air at ambient pressure and temperature, Fig. 54. The shrinkage is caused by the capillary pressure exerted on the compliant gel network as the pore fluid evaporates: 


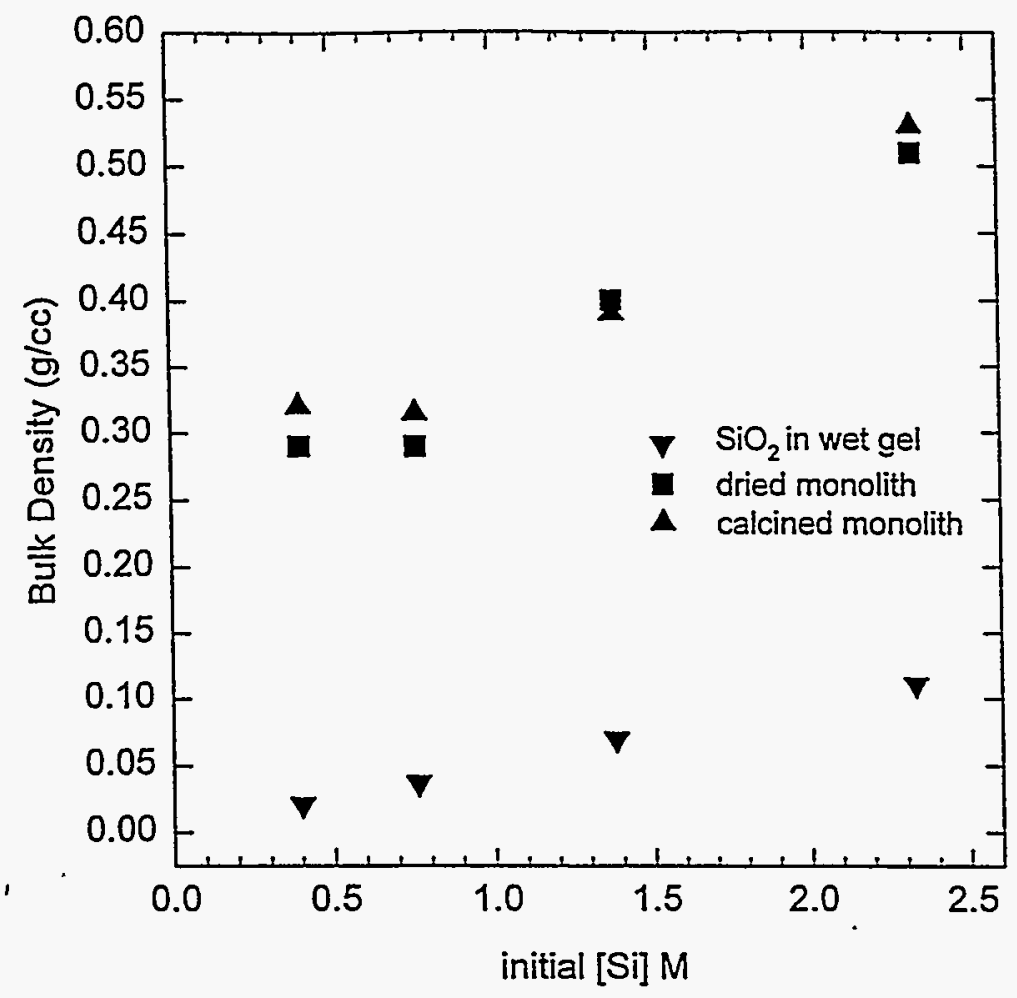

Figure 53. Bulk density increases approximately linearly with initial silica concentration for PMSG monoliths. Samples were dried in air from 75:25 water:methanol pore fluid and calcined in $\mathrm{N}_{2}$ followed by $\mathrm{O}_{2}$.

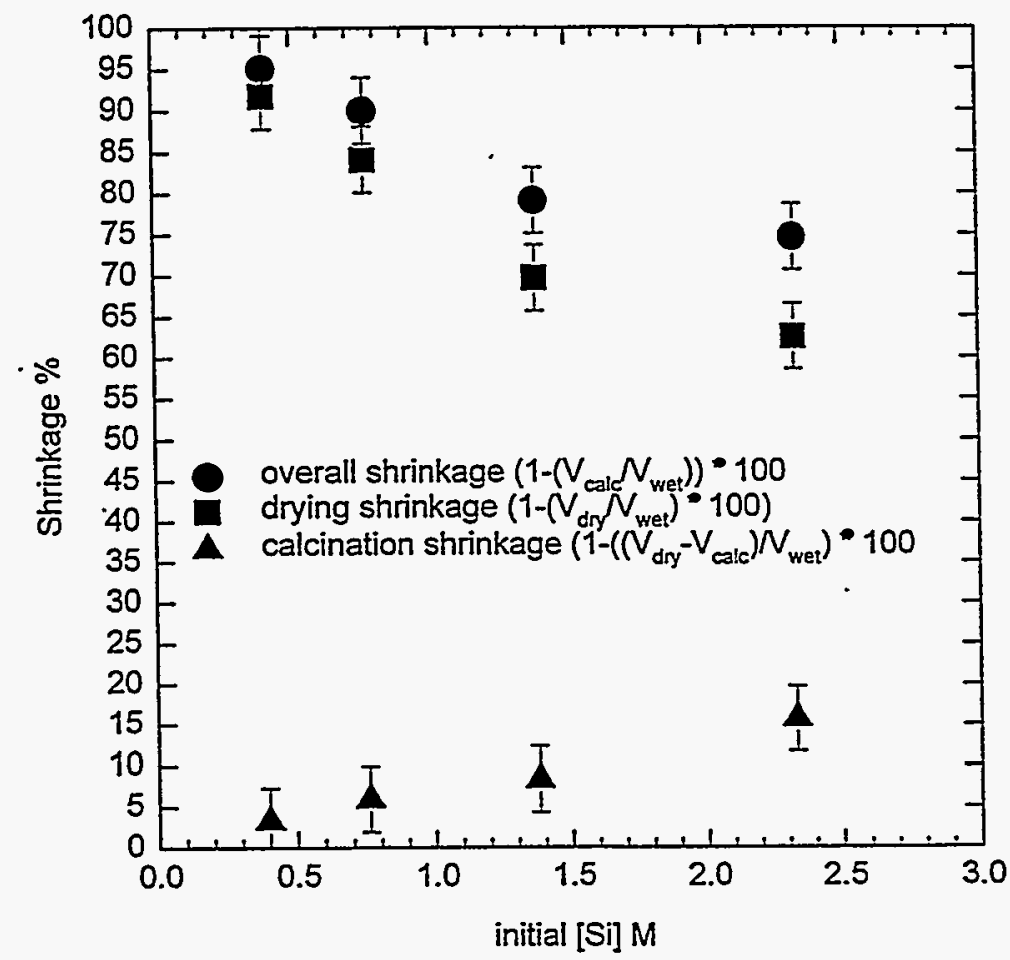

Figure 54. Drying and overall shrinkage of monoliths decrease approximately linearly with initial silica concentration. Calcination shrinkage, relative to the dried volume ( 1 $\left.\left(\mathrm{V}_{\text {calc }} / \mathrm{V}_{\mathrm{dry}}\right) * 100\right)$ remains constant at about $40 \%$ (not shown). 


$$
P_{c}=-2 \gamma_{L V} \cos \theta / a
$$

where $P_{c}$ is the capillary pressure, $\gamma_{L V}$ is the pore fluid surface tension, $\theta$ is the contact angle between the pore fluid and the pore wall, and $a$ is the pore radius.

The capillary forces generated by the pore fluid in the interparticle voids exert a tremendous tension on the gel network, and this increases the bulk densities of the monolithic gels to $\sim 0.29 \mathrm{~g} / \mathrm{cc}$ for the [Si] = $0.4 \mathrm{M}$ sample to $\sim 0.51 \mathrm{~g} / \mathrm{cc}$ for the $[\mathrm{Si}]=2.33 \mathrm{M}$ sample. The intraparticle channels contribute very little to the shrinkage: Even though within each particle there are $\sim 3$ $\mathrm{nm}$ channels that could be subject to a large capillary pressure, the channels are filled with surfactant template and the capillary pressure exerted by the small amount of polar fluid in the hydrophobic channels is expected to be minimal. In fact $X$-ray diffraction measurements of the pore diameter do not change, within the error of the experiment, on drying the gel.

The drying shrinkage can be significantly reduced by exchanging the pore fluid with one that has a lower surface tension. For example, the drying shrinkage for an acetone exchanged [Si] $=0.4 \mathrm{M}$ gel is only $\sim 30 \%$ compared to $\sim 85 \%$ for $75: 25$ water:methanol. In fact, the very low theoretical densities $(\sim 0.02$ to $0.20 \mathrm{~g} / \mathrm{cc})$ for the wet gels suggest that if the pore fluid were supercritically extracted (it would be desirable to exchange the water:methanol mixture for a fluid with a lower critical pressure) a low density, aerogel type materials would result. Supercritical drying studies are in progress.

D.5 Accessing Intraparticle Porosity - The intraparticle porosity is accessed by carefully calcining the monolith to remove the surfactant template. When the template is removed from the dried gel, an additional shrinkage occurs, Fig. 53, but the bulk density remains nearly constant Fig. 54 . The constant density is a fortuitous balance of the weight loss owing to pyrolysis - which decreases the density - and the volume contraction owing to particle 


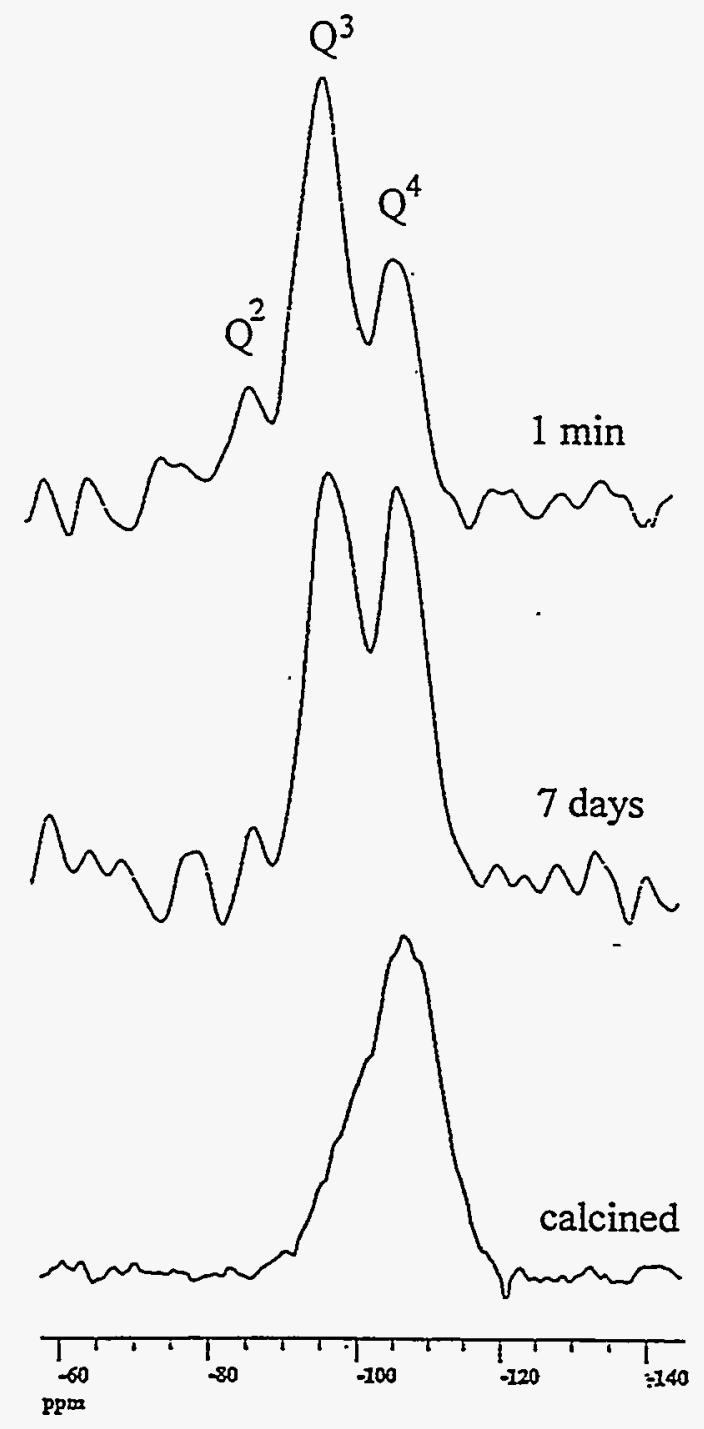

Figure 55. Deconvolution and integration of ${ }^{29} \mathrm{Si} N M R$ data on gel samples isolated after 1 minute indicate $Q^{3} / Q^{4}$ " 2.1 ; after 7 days the ratio is $\sim 1.2$; after calcination the ratio is $\sim 0.4$.

shrinkage - which tends to increase the density. The experimental weight loss during calcination is $\sim 40 \%$ regardless of initial silica concentration and the volume shrinkage at $550{ }^{\circ} \mathrm{C}$ is also $\sim 40 \%$ (relative to the dried volume) regardless of initial silica concentration.

The shrinkage of the gel during calcination can be almost solely attributed to a shrinkage of the primary particles of the gel. $X$-ray diffraction data on crushed gels that have been calcined indicate the unit cell constant of the silica shrinks $\sim 10 \%$ to $20 \%$ depending on exact experimental conditions. 
If we assume that the shrinkage of the particles is essentially isotropic (which certainly does not have to be the case in an orthotropic system) and we calculate the particle volume shrinkage based on the cell shrinkage, we obtain a $\sim 27$ to $\sim 49 \%$ volume shrinkage for the particles ( $x^{3}$ dependence). The observed $\sim 40 \%$ shrinkage fits nicely in this range.

In the early, low temperature stage of calcination, the removal of adsorbed solvent and water vapor from the particles drives shrinkage through the capillary forces exerted on the pores. This effect is opposed by the coefficient of thermal expansion of the silica framework. The shrinkage (or expansion) in this stage of calcination is typically small in amorphous silica. At slightly higher temperatures (e.g. $>150^{\circ} \mathrm{C}$ ) the template is pyrolyzed $(\sim 300$ ${ }^{\circ} \mathrm{C}$ ) and the framework continues to polymerize; the $Q^{3} / Q^{4}$ ratio decreases from $\sim 2.1$ in the wet gel to $\sim 0.4$ in the calcined gel, Fig. 55. The increased condensation of silanol groups to form siloxane bonds, which is thermodynamically and/or kinetically driven by the elimination of the excess free volume created by removal of the organic templates, probably account for the bulk of the shrinkage observed during calcination. Note that whereas drying the monolith primarily affects the interparticle porosity, removing the template affects both the inter- and intraparticle porosities.

D.6 Measuring Inter- and Intraparticle Porosity - Calcined PMSGs exhibit sorption isotherms, Fig. 56, that indicate a bimodal pore size distribution, Fig. 57. Similar to bulk materials, they show distinctive nitrogen isotherms that exhibit pronounced uptake of gas over a very narrow range of $P / P$ 。 corresponding to a narrow distribution of intraparticle mesopores (typically 25 $\AA$ in diameter). In addition, they show a broad hysteretic uptake of nitrogen at high $P / P_{o}$, which corresponds to a broad distribution of interparticle pores (typically $10-50 \mathrm{~nm}$ in diameter). The total pore volume is 1.6-2.9 cc/g $\left(V_{p}=1 / \rho_{\text {bulk }}-1 / \rho_{\text {framework }}\right.$ where $\rho_{\text {framework }}$ is $\left.\sim 2.3 \mathrm{~g} / \mathrm{cc}\right)$ of which about $0.3-0.6 \mathrm{cc} / \mathrm{g}$ is intraparticle and $1.3-2.3 \mathrm{cc} / \mathrm{g}$ is interparticle. 


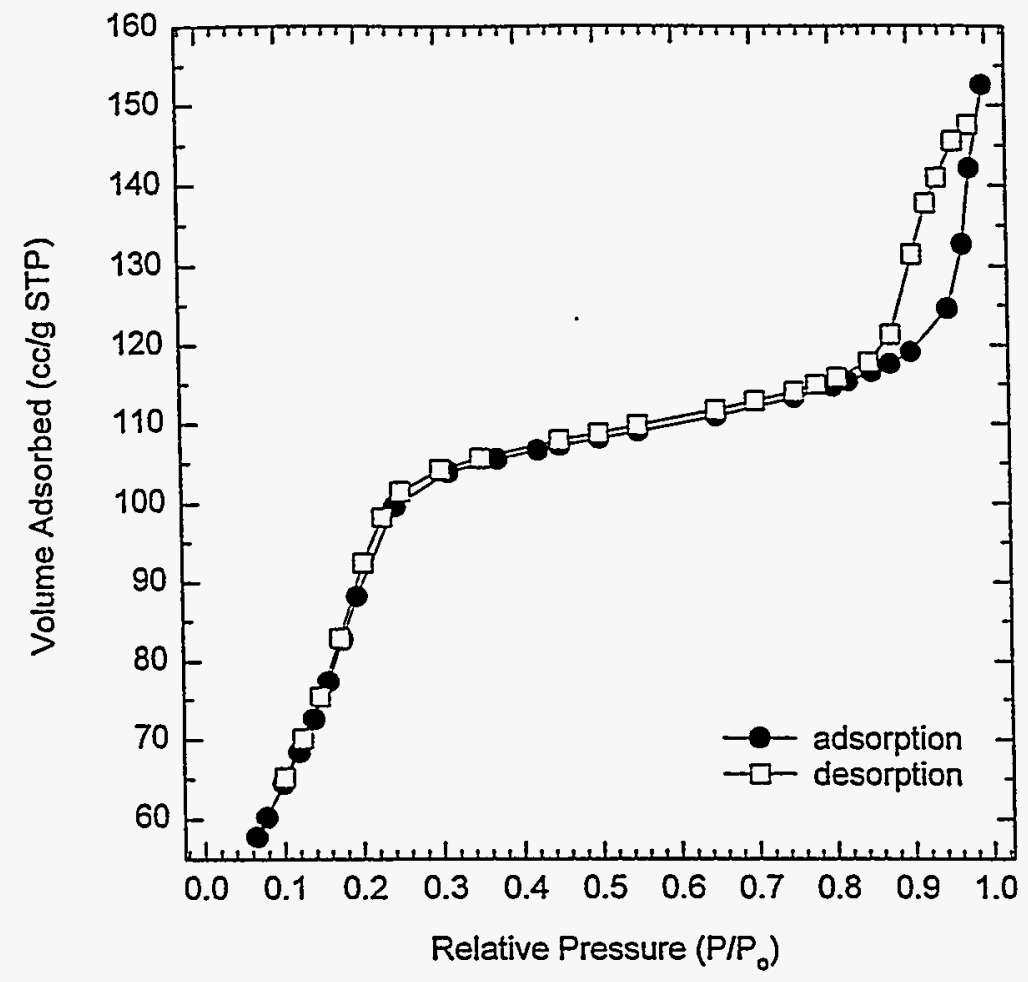

Figure 56. $\mathrm{N}_{2}$ Sorption $77^{\circ} \mathrm{K}$ shows an inflection at $\mathrm{P} / \mathrm{P}_{\mathrm{o}} \gg 0.15$, indicating uniform $-2.0 \mathrm{~mm}$ intraparticle mesopores, and shows a hysteresis at high $P / P_{0}$, indicating large interparticle pores.

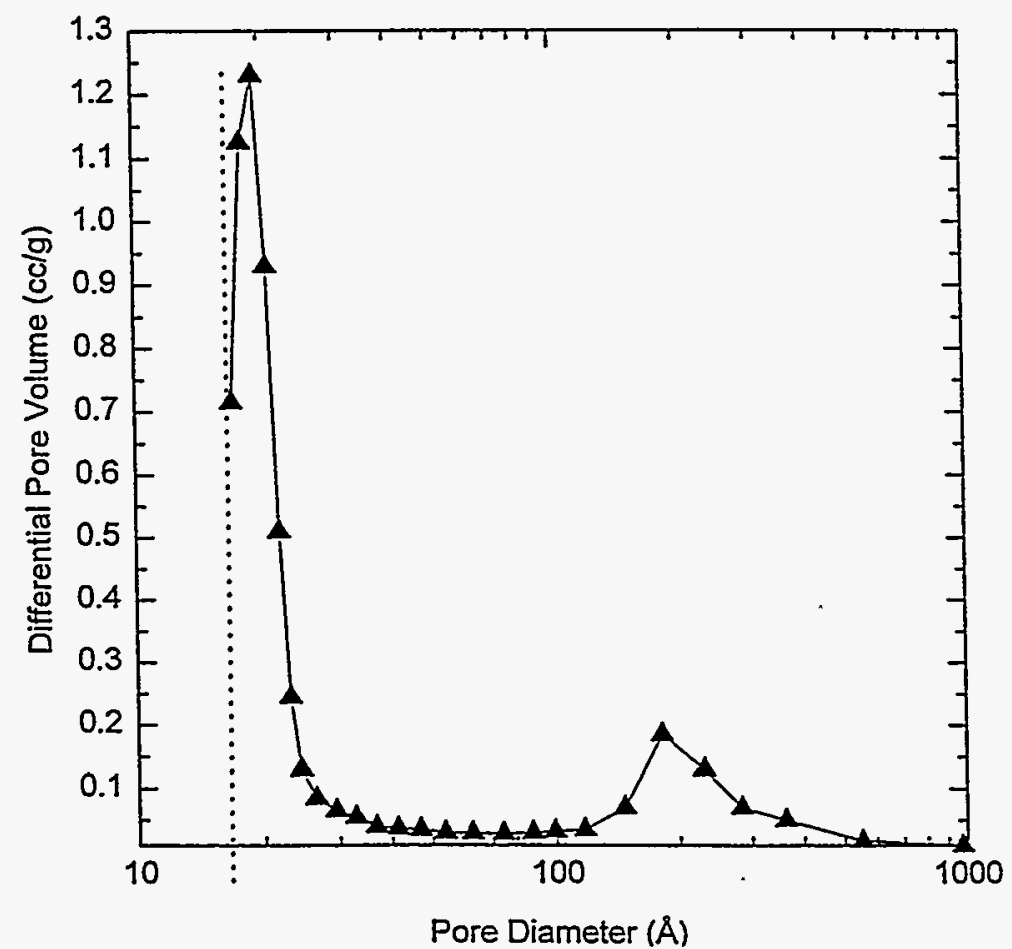

Figure 57. BJH differential pore volume indicates a bimodal distribution of pores with narrow intraparticle mesopores with $\mathrm{d}_{\max }=20 \AA$ and a broader set of pores with $\mathrm{d}_{\max }-180 \AA$. 


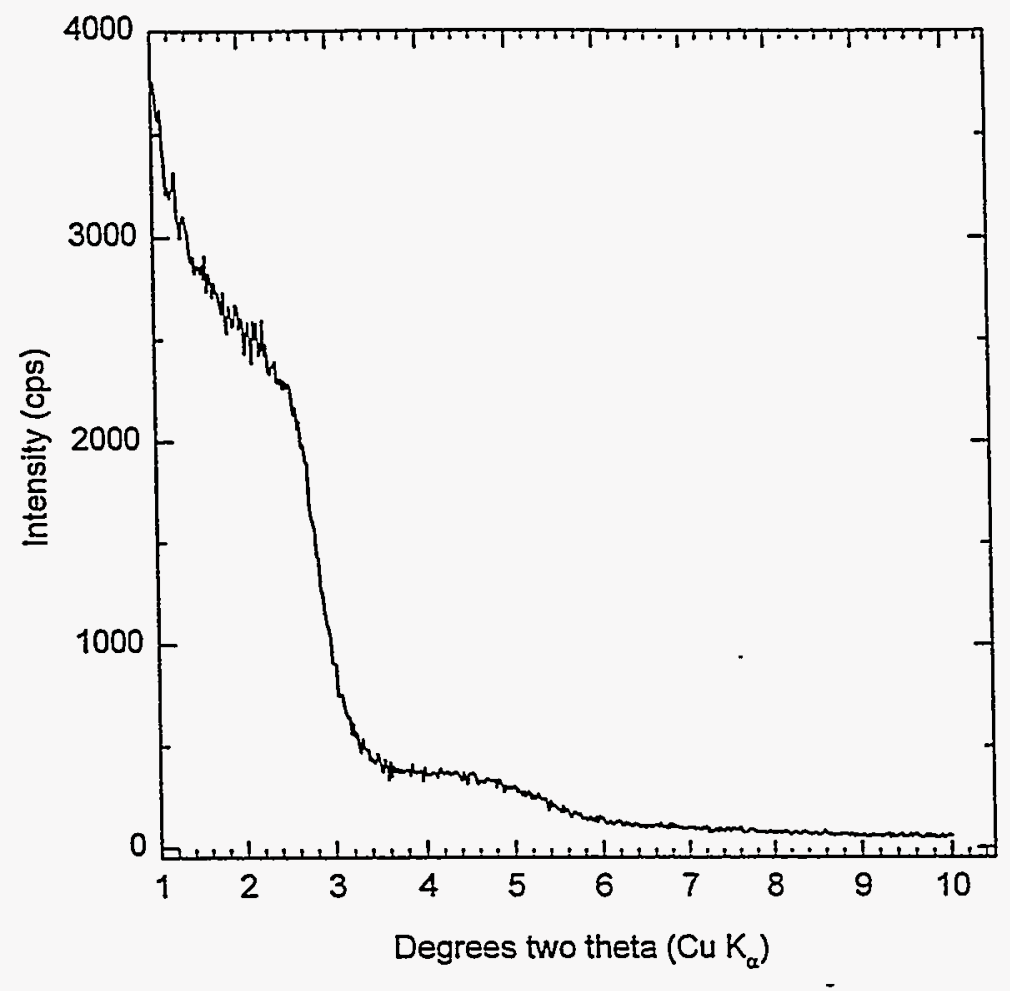

Figure 58. X-ray diffraction data on calcined monolith (crushed after calcination) exhibits two broad peaks which indicates the long range order of pores is significantly less than in the samples that were crushed and then calcined.

The $X$-ray diffraction patterns of the calcined monoliths, Fig. 58, exhibit only one diffraction peak compared to 4 or 5 for the powders, Fig. 52. This indicates the indicates the long-range order of the of pore system is less in the monoliths; however the FWHM of the pore diameter distributions as determined from $\mathrm{BJH}$ analysis of the desorption branch of the isotherm are quite comparable ( $d V / d R \sim 6-7 \AA$ for the monoliths and $d V / d R \sim 4-6 \AA$ for the powders). This implies that the registry of the pores is slightly compromised in the calcined monoliths, but that the unimodal, narrow intraparticle pore size distribution remains.

There is a report [44] of a silica material that contains two broad distributions of pore sizes; $d_{\text {ave }}=\sim 30 \AA$ (FWHM $\sim 15 \AA$ ) and $d_{\text {ave }}=\sim 110 \AA$ (spread from 80 to $200 \AA$ ) that was synthesized by calcining an $X$-ray amorphous wet product derived from tetraethoxysilane (TEOS) and cetyltrimethylammonium chloride (CTAC). Quite different from TMOS 
derived gels, the sorption isotherm for this material shows no discernible uptake of nitrogen in the $\mathrm{P} / \mathrm{P}_{\mathrm{o}}$ region that would correspond to $30 \AA$ pores.

\section{E. Conclusions}

We have developed a synthetic method to form novel mesoporous periodic silica gel monoliths and pellets of arbitrary shape and size. The key to gel formation is the silica source, TMOS, the rapid kinetics associated with product formation, and the high degree of $Q^{3}$ species present at the gel point. The large pore volumes, surface areas, and well defined intraparticle porosity may make PMSGs useful as high capacity storage materials for condensable gases, such as methane and other light hydrocarbons, as graded particle size/graded pore size separation media for liquid chromatography, or a nanoscale reaction vessels for quantum confined clusters. As made from laboratory reagents, the cost for dried and calcined PMSG is a prohibitive $\sim 0.60 / \mathrm{cc}$. By using reagents in bulk the cost could be lowered significantly. Future work will center on understanding how the choice and concentration of cosolvent can be used to tailor the properties of these materials. 


\section{Effects of Cosolvents, Swelling Agents and Added Salt}

\section{A. Epitome}

We have examined the effects of fourteen cosolvents of varying dielectric constant, hydrogen bonding capacity, and electron donating ability on the solution homogeneity, micellization, periodicity, gel time, pore diameter, and crystallite size of periodic mesoporous silica. We find that a wide variety of cosolvents act as homogenizing agents; that cosolvents decrease the extent of micellization; that the cosolvent dielectric constant (polarity) and concentration determines the degree to which micellization is reduced; that product periodicity is intimately linked to micellization; that we can make periodic products with water:cosolvent ratios as low as $3: 97 \mathrm{w} / \mathrm{w}$, which corresponds to a water to silica ratio of $\sim 3.9$; that the gel time is essentially insensitive to cosolvent concentration as long as the surfactant concentration exceeds the critical micelle concentration ( $\mathrm{cmc}$ ); that all cosolvents affect the pore diameter and can be used to continuously tune the pore aperture over several angstroms; and that crystallite size and dispersity is only slightly affected by cosolvent type. We also made mesophases from oil swollen micelles (microemulsions) and examined the changes in pore diameter and periodicity. We find that swollen mesophases can be made from microemulsions, but that the order parameter of the 2-D pore array is very small. Finally, we examined the effect of added salt on pore size, gel time, periodicity, and crystallite size. We find that multidentate, multicharged salts in high concentration can increase pore diameter, increase gel time, and decrease periodicity.

\section{B. Introduction}

It has recently been discovered that liquid crystalline surfactant templates can be used to synthesize periodic porous metal oxides. In this 
process, a source of the metal oxide is added to an acidic or basic aqueous micellar solution [1-6]. Small metal oxide ions are then generated by dissolution or hydrolysis of the metal oxide source. These ions displace the counterions that surround the micelle and induce a transformation to liquid crystalline metal oxide/surfactant microdomains. These domains grow to form particles that either precipitate from solution or form an aggregate gel. To access the porosity, the template can then removed from the isolated product by calcination or liquid extraction.

Because the template is an supramolecular aggregate, rather than a single molecule, as in the synthesis of periodic microporous oxides such as zeolites, the characteristics of the template can be greatly affected by the properties of the solvent. For example, there is a vast body of literature [47] on micellar solutions that shows that the micelle structure, curvature, aggregation number and volume; the extent of micellization; and the interfacial solvent-micelle tension can be controlled by adding low dielectric constant oils or electrolytes. In addition, there are many studies of how these physical properties are altered in completely nonaqueous systems. [48,49]

We have previously studied the effects of adding the cosolvent methanol to a purely aqueous system and found that we could control the size of the template, the extent of micellization, and the periodic domain size [33]. Based on this work, and the literature on micellization, we thought it was likely that by adding (1) nonaqueous cosolvents, (2) swelling agents, (3) electrolytes to our standard aqueous sysnthesis, we could contol the chemistry of the precursors and inorganic framework (e.g. fraction of unreacted silanol groups); the structure (i.e. hexagonal, lamellar, cubic) of the mesophase; the pore diameter and pore size distribution; and perhaps even the large scale particle size and shape.

Our initial objective in using methanol as a cosolvent was to rapidly dissolve our hydrophobic silica source, tetramethoxysilane (TMOS) in a substantially aqueous environment. It is well known that cosolvents such as alcohols act as homogenizing agents for alkoxides in aqueous systems, and 
this works in micellar solutions as well. However, we found that methanol also has a dramatic effect on micellization and the final mesophase, and thus thought that other polar cosolvents might also be useful in controlling the product synthesis and structure [33]. Adding miscible cosolvents to purely aqueous solutions changes the solution thermodynamics, generally decreasing the extent of micellization (i.e. increases the critical micelle concentration) and decreasing the aggregation number [47]. It is perhaps surprising that liquid crystalline phases of cetyltrimethylammonium bromide (CTAB) form in several highly polar solvents (glycerol, formamide, ethylene glycol, and $\mathrm{N}$-methylformamide) [48,49], indicating the possiblity of a substantially nonaqueous synthesis of mesoporous silica.

Based on these observations we pursued three lines of investigation: (1) to determine if nonaqueous synthesis of periodic silica is possible; (2) to determine the effect of decreased micellization on product periodicity; and (3) to determine the effect of cosolvent concentration on pore diameter.

Cosolvents also aid in synthesizing heteronuclear periodic mesophases, by enabling the premixing and prehydrolyzation of metal alkoxides in a substantially nonaqueous environment. The resulting $\mathrm{M}-\mathrm{O}-$ $\mathrm{M}^{\prime}$ precursor crosslinking should improve the homogeneity of frameworks.

Water immiscible solvents act as micelle swelling agents, not cosolvents, and can be used to control the pore size. Previous work has shown that pore size can be continuously tuned from $\sim 40$ to $100+\AA$ by dissolving oils into a freshly synthesized, but incompletely condensed mesophase [1,4]. The oil simply swells the nascent framework, preserving the periodicity. However, it has been found that adding the oil prior to product formation results in poorly ordered materials. Using light scattering we have found that these microemulsions are very polydisperse, and we believe this accounts for the poor product periodicity.

We also investigated the effect of added salt on product formation. A substantial literature [47] has shown that increasing the ionic strength of micellar solutions tends to decrease the critical micelle concentration $(\mathrm{cmc})$ 
and increase the micelle aggregation number. The increased charge screening provided by high electrolyte concentration has also been implicated in the several order of magnitude decrease in the gelation kinetics in silica systems [46]. We find that highly charged, multidentate ions can actually increase the gel time and pore diameter when present in high concentrations.

\section{Experimental}

C.1 Synthesis - Our work focused on 1-d hexagonally arrayed periodic mesoporous silica. A typical synthesis involved mixing: $9.8 \mathrm{~g}$ deionized water/cosolvent, $0.2 \mathrm{~g}(0.55 \mathrm{mmol})$ of cetyltrimethylammonium bromide, (CTAB; Fisher, 99+\%), $0.065 \mathrm{~mL} 50 \mathrm{wt} \% \mathrm{NaOH}(1.14 \mathrm{mmol})$, and $0.625 \mathrm{~mL}$ (4.23 mmol) tetramethoxysilane (TMOS, United Chemical Technologies). Molar ratios of CTAB:NaOH:TMOS in the initial mixture were 0.13:0.27:1. Water to silicon ratios varied from 129:1 to < 4:1.

A variety of simple ethers, esters, carboxylic acids, glycols, ketones, amides, nitriles, and alcohols were used as cosolvents, Table I. These include polar and nonpolar, protic and aprotic, donating and nondonating solvents. Samples were typically made with 100:0, 90:10, ... 10:90 water:cosolvent ratios. Smaller increments over limited ranges were examined in several cases.

A variety of low dielectric constant swelling agents including carbon tetrachloride, cyclopentane, benzene, toluene, 1,3,5-trimethylbenzene, 1bromo2,3-dichlorobenzene, and dodecane were used as swelling agent:silica molar ratios up to 5.7:1. These oils were added to the micellar solutions to make microemulsions. Several salts including $\mathrm{KI}, \mathrm{K}_{2} \mathrm{SO}_{4}, \mathrm{NaCl}, \mathrm{NaH}_{2} \mathrm{PO}_{4}$ (titrated to $\mathrm{pH} 13$ with $\mathrm{NaOH}$ ), and $\mathrm{K}_{2} \mathrm{C}_{2} \mathrm{O}_{4}$ were used in concentrations up to $2.5 \mathrm{M}$. Salts were added to the micellar solutions prior to the addition of the TMOS.

Adding the TMOS to the precursor solutions results in weak particulate gels. After aging ( $1 \mathrm{~min}$ to $30 \mathrm{~d}$ ) the wet silica/surfactant gels were suction filtered, washed with deionized water and/or methanol and dried in 
Table I. Physical Properties of Cosolvents

\begin{tabular}{|l|l|c|c|c|c|}
\hline cosolvent & formula & polar & protic & donor & $\varepsilon\left(25^{\circ} \mathrm{C}\right)$ \\
\hline ether & $\mathrm{H}_{5} \mathrm{C}_{2} \mathrm{OC}_{2} \mathrm{H}_{5}$ & & & $\mathrm{x}$ & 4.34 \\
\hline ethyl acetate & $\mathrm{CH}_{3} \mathrm{C}=\mathrm{OOC}_{2} \mathrm{H}_{5}$ & & & $\mathrm{x}$ & 6.0 \\
\hline THF & $\mathrm{C}_{4} \mathrm{H}_{4} \mathrm{O}$ & & & $\mathrm{x}$ & 7.6 \\
\hline methylene chloride & $\mathrm{H}_{2} \mathrm{CCl}_{2}$ & & & & 8.9 \\
\hline isopropanol & $\mathrm{C}_{3} \mathrm{H}_{7} \mathrm{OH}$ & $\mathrm{x}$ & $\mathrm{x}$ & $\mathrm{x}$ & 18.3 \\
\hline acetone & $\mathrm{CH}_{3} \mathrm{C}=\mathrm{OCH}_{3}$ & $\mathrm{x}$ & & $\mathrm{x}$ & 21 \\
\hline ethanol & $\mathrm{C}_{2} \mathrm{H}_{5} \mathrm{OH}$ & $\mathrm{x}$ & $\mathrm{x}$ & $\mathrm{x}$ & 25 \\
\hline methanol & $\mathrm{CH}_{3} \mathrm{OH}$ & $\mathrm{x}$ & $\mathrm{x}$ & $\mathrm{x}$ & 33 \\
\hline ethylene glycol & $\mathrm{C}_{2} \mathrm{H}_{6} \mathrm{O}_{2}$ & $\mathrm{x}$ & $\mathrm{x}$ & $\mathrm{x}$ & 37 \\
\hline acetonitrile & $\mathrm{CH}_{3} \mathrm{CN}$ & $\mathrm{x}$ & $\mathrm{x}$ & $\mathrm{x}$ & 43 \\
\hline glycerol & $\mathrm{CH}_{2} \mathrm{OHCHOHCH} \mathrm{OH}_{2} \mathrm{OH}$ & & $\mathrm{x}$ & $\mathrm{x}$ & 7.7 \\
\hline tetraglyme & $\mathrm{CH}_{3}\left(\mathrm{OCH}_{2} \mathrm{CH}_{2}\right)_{4} \mathrm{OCH}_{3}$ & $\mathrm{x}$ & $\mathrm{x}$ & $\mathrm{x}$ & 78 \\
\hline water & $\mathrm{H}_{2} \mathrm{O}$ & $\mathrm{x}$ & $\mathrm{x}$ & $\mathrm{x}$ & 111 \\
\hline formamide & $\mathrm{HC}=\mathrm{ONH}_{2}$ & $\mathrm{x}$ & $\mathrm{x}$ & $\mathrm{x}$ & 188 \\
\hline $\begin{array}{l}\mathrm{N} \text {-methyl } \\
\text { formamide }\end{array}$ & ${\mathrm{HC}=\mathrm{ONHCH}_{3}}$ & & & & \\
\hline
\end{tabular}

NOTE: $\varepsilon$ - dielectric constant

air. The surfactant typically was removed from the product by calcination. The calcination process involved a linear temperature ramp from 25 to $550{ }^{\circ} \mathrm{C}$ over $2-10 \mathrm{~h}$ flowing $\mathrm{N}_{2}$; an isotherm at $550^{\circ} \mathrm{C}$ for $1-2 \mathrm{~h}$; cooling to $<300{ }^{\circ} \mathrm{C}$; a linear ramp to $550^{\circ} \mathrm{C}$ in flowing $\mathrm{O}_{2}$; an isotherm for 6-10 h; and cooling to room temperature in flowing oxygen (furnace off). The calcined product was usually pristine white, if not, it was again calcined in $\mathrm{O}_{2}$ at $550^{\circ} \mathrm{C}$ for $\sim 10 \mathrm{~h}$.

C.2 X-ray Diffraction - X-ray data were collected with a Scintag PAD V instrument using nickel-filtered $\mathrm{Cu} \mathrm{K}_{\alpha}$ radiation. Data were collected in continuous scan mode from 1.5 to $10^{\circ} 2 \theta$ with a $0.02^{\circ}$ sampling interval and a $1 \%$ min scan rate. Slits widths starting from the source were $1,2,1$, and 0.3 $\mathrm{mm}$. Tube voltage was $45 \mathrm{kV}$ and tube current was $35 \mathrm{~mA}$. Peak positions and full-widths at half maxima were determined with Scintag analysis software (TC9 package). Peak positions for the periodic hexagonal phase were 
corrected with an external standard routine. The routine used four Bragg peaks $(100,110,200,210)$ for the correction. Linear least squares analysis of the peak positions yielded accurate lattice parameters.

C.3 SEM/TEM - A JOEL 1200EX transmission electron microscope (TEM) with ASID (SEM) attachment was used to observe the microstructure and grain size of the powder samples. In order to observe individual grains, the aggregated powders were ground under methanol in a mortar and pestle. The suspended powder was caught on the holey carbon film of a $3 \mathrm{~mm}$ copper grid. Bright field TEM or diffraction contrast imaging was done at $120 \mathrm{kV}$ and involved low (20k times) and high (300k times) magnifications of the individual grains and small aggregates. SEM micrographs were obtained that revealed the morphology of powder aggregates and the size of the grains.

C.4 Gas Adsorption - A Micromeritics ASAP 2010 was used to collect $\mathrm{N}_{2}$ sorption data at $77^{\circ} \mathrm{K}$. Samples were degased overnight at $200^{\circ} \mathrm{C}$. BET analyses were performed to determine the apparent surface areas. Pore diameters were determined by application of the Kelvin Equation to the desorption branch of the data.

\section{Results and Discussion}

D.1 Cosolvents and the Hexagonal Existence Region (ERHM) - In general, we find that four types of products can be formed from water:cosolvent solutions. These materials are characterized by their X-ray diffraction patterns: (1) hexagonal mesophase structures $(H)$ that exhibit at least 3 low angle diffraction peaks (indexed as the 100, 110, and 200 on a 2-d hexagonal net), (2) disordered mesophase structures (D) that exhibit only one diffraction peak and that are, in most cases, materials with a disordered arrangement of cylindrical 1-d pores (quasi hexagonal), (3) lamellar mesophase structures (L) characterized by a family of $00 l$ diffraction peaks, and (4) amorphous silica (A), 
which exhibit no low angle Bragg peaks. We define the ERHM as the range of $r$-where $r$ is the weight percent cosolvent in a water:cosolvent mixture - for which an $\mathrm{H}$ product is observed.

The ERHM was examined for each cosolvent by varying $r$, Fig. 59 . For cosolvents with dielectric constants $(\varepsilon)$ less than 10 , the existence region is quite small $(0 \% \leq r<20 \%)$, Figs. 60-64. The nonpolar cosolvents diminish the ability of the surfactant to act as a chemical dipole; the interaction of the hydrophobic tails and the solvent is less unfavorable, which decreases the energetic favorability of forming micelles. This limits the formation of periodic hexagonal mesophases from mixed water:nonpolar solvent systems to substantially aqueous environments.

In some low dielectric constant cosolvents, such as diethyl ether, methylene chloride and THF, the lamellar phase becomes much more favorable. In these solvents the structure of the product changes from hexagonal to lamellar ( $\mathrm{H}$ to $\mathrm{L}$ ) with increasing $r$. This transition occurs at $r \cong$

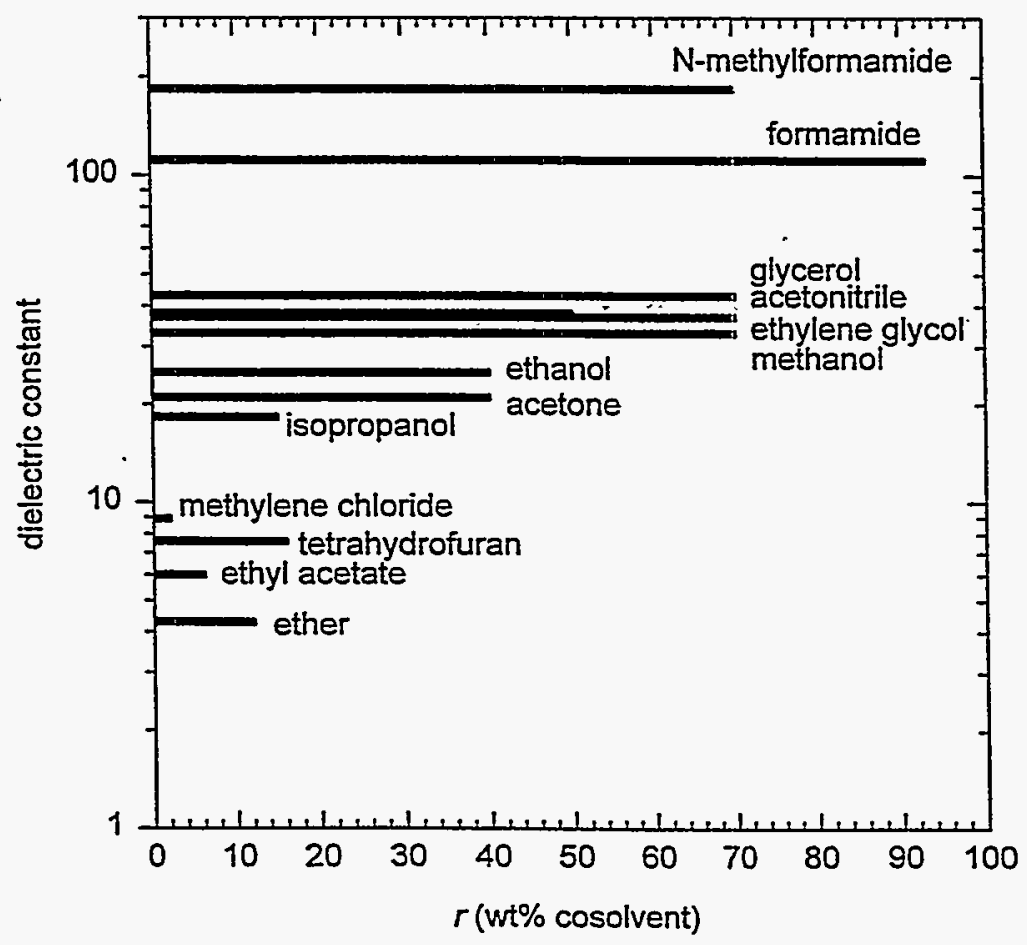

Figure 59. The existence region of hexagonal mesophases (ERHM) is shown for various cosolvents. The ERFM is defined as the range of $r$ values for which hexagonal mesophases with at least three diffraction peaks $(100,110$, and 200) form. The ERFM generally increases with dielectric constant. 


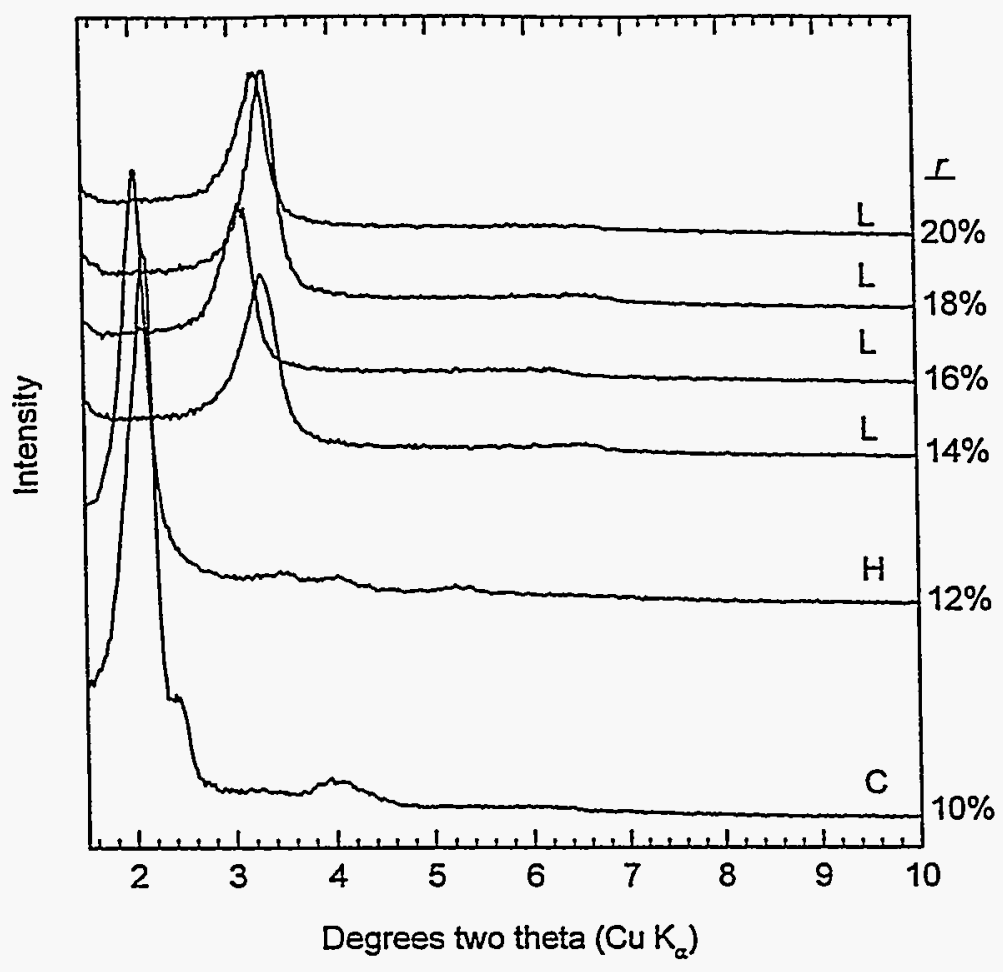

Figure 60. X-ray diffraction patterns for the water:diethyl ether system show the transformation from cubic to hexagonal to lamellar with increasing $r$. The samples were aged 5 days.

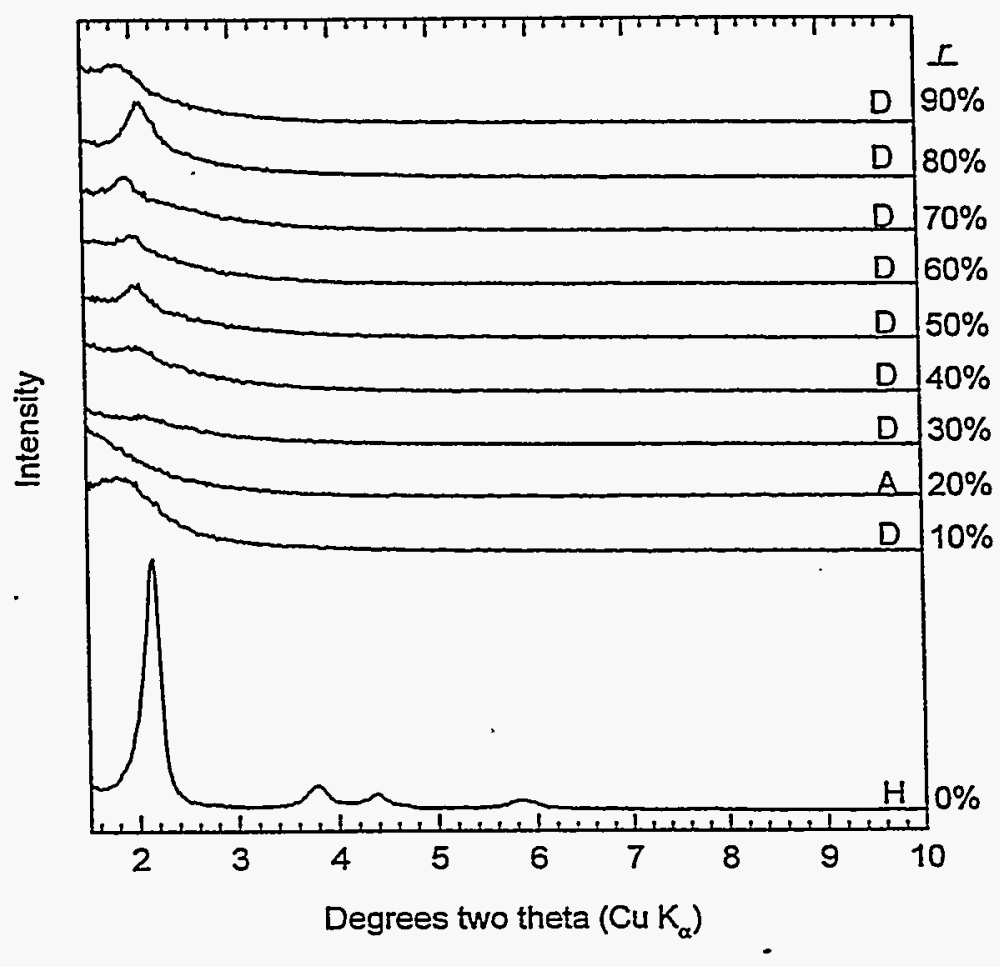

Figure 61. X-ray diffraction patterns for the water:ethyl acetate system show the transformation from hexagonal to disordered to amorphous and back to disordered. The samples were aged 1 day. 


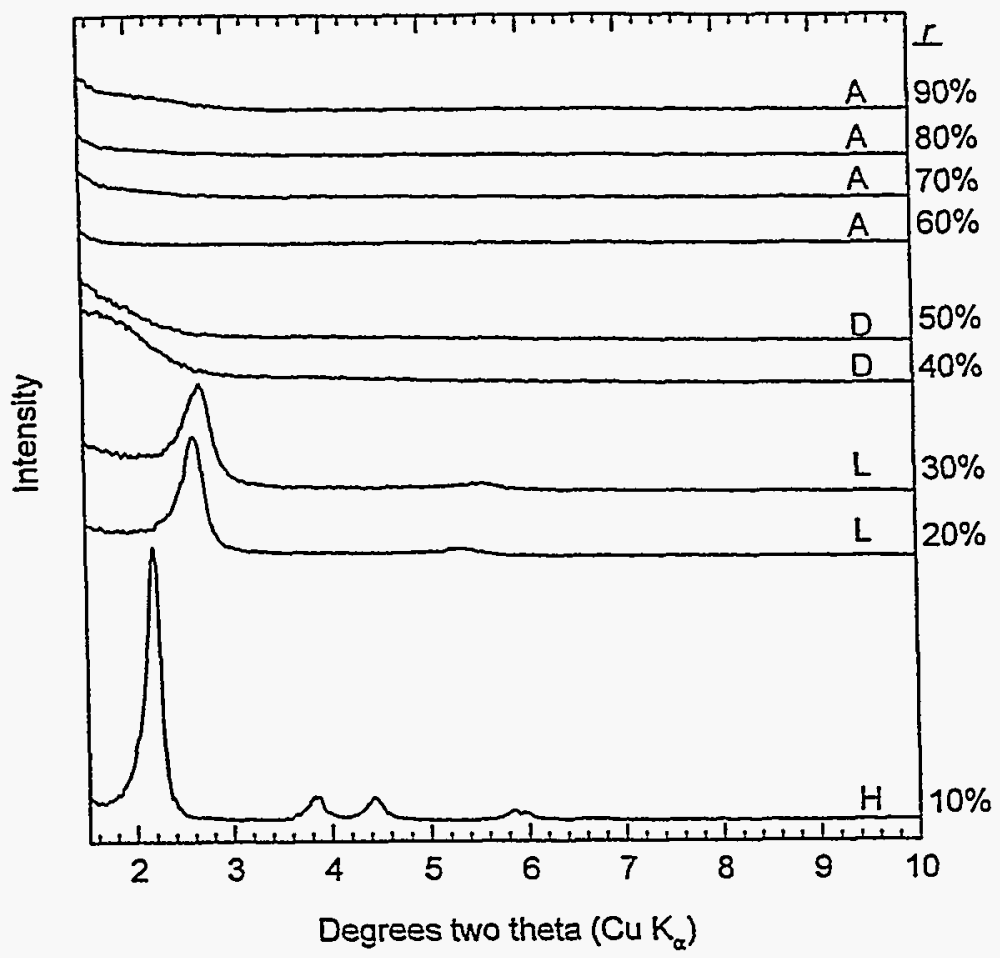

Figure 62. X-ray diffraction patterns for the water:tetrahydrofuran system show the transformation from hexagonal to lamellar to disordered to amorphous as $r$ increases. The samples were aged 4 days.

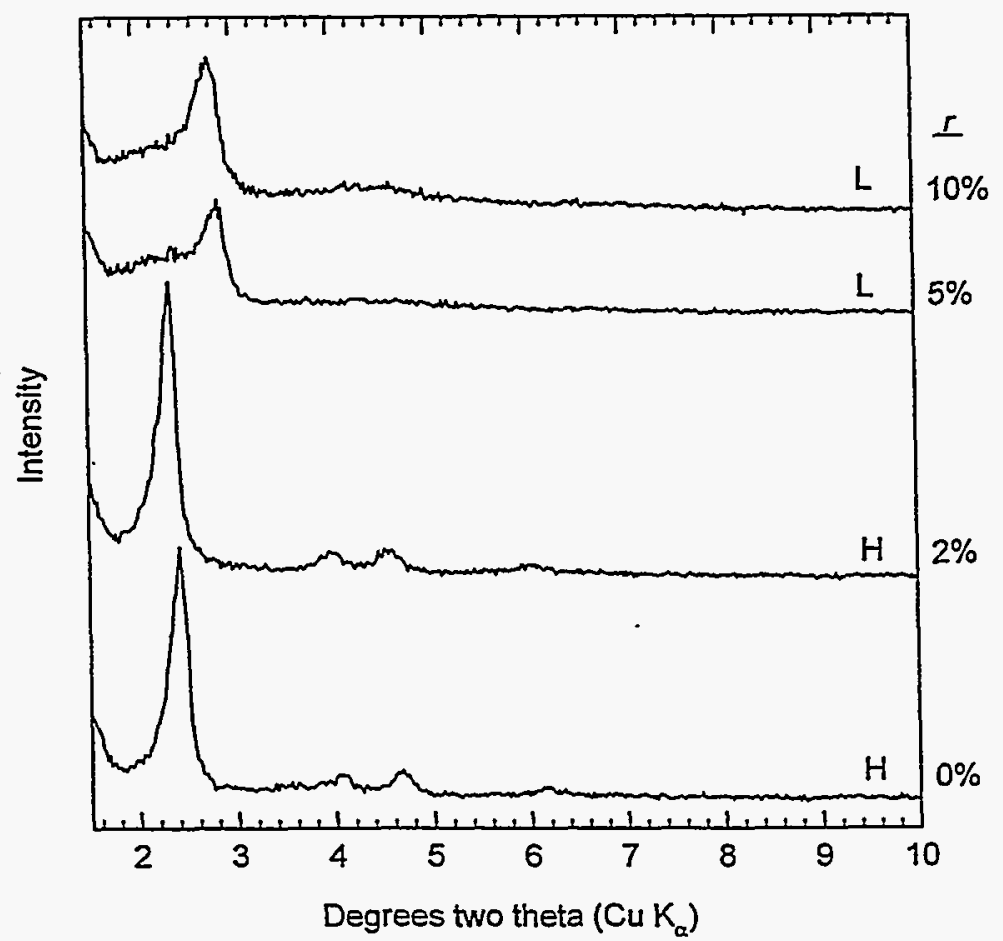

Figure 63. X-ray diffraction patterns for the water:methylene chloride system show the transformation from hexagonal to lamellar as $r$ increases. The solvent system phase separates at $r=5 \%$. The samples were aged 10 minutes. 


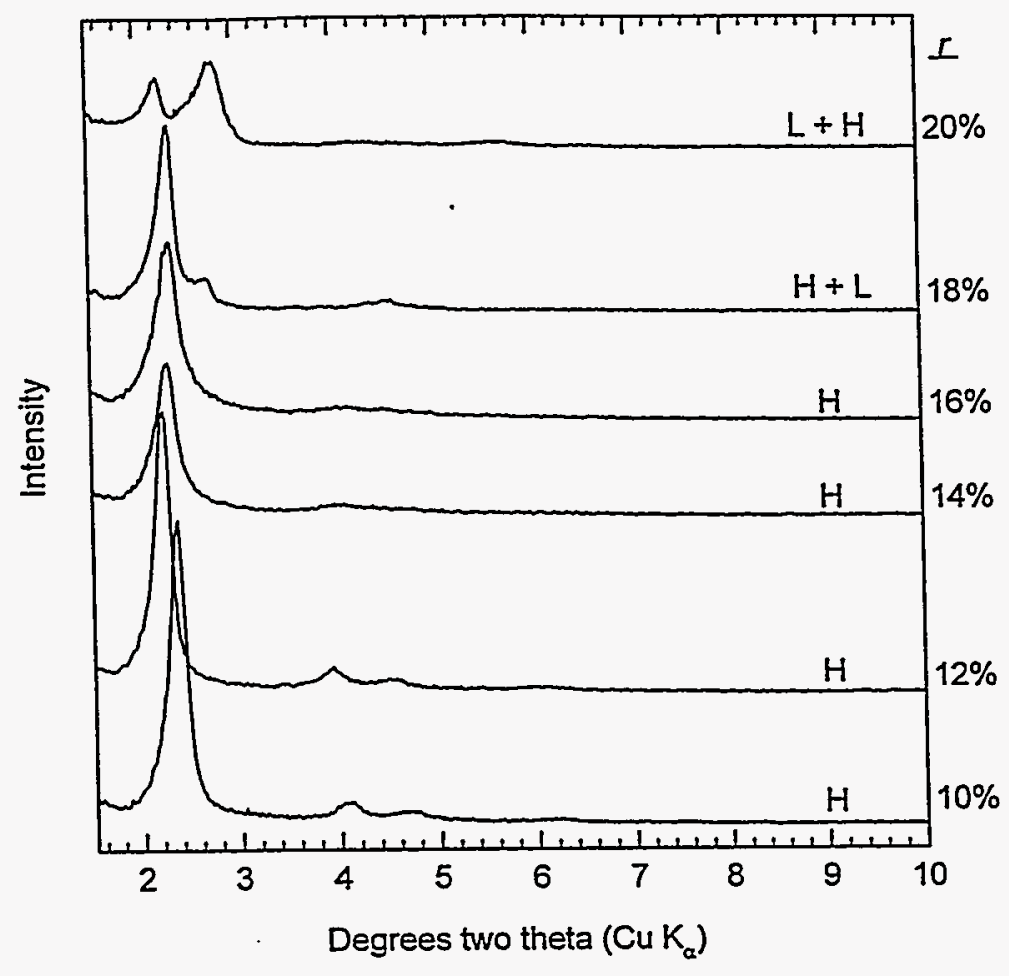

Figure 64. X-ray diffraction patterns for the water:tetrahydrofuran system show the transformation from hexagonal to lamellar at $\mathrm{r} \approx 18 \%$. The transformation is incomplete for the aging time of 3 days. The samples yellow after aging, which may indicate some decomposition of the surfactant.

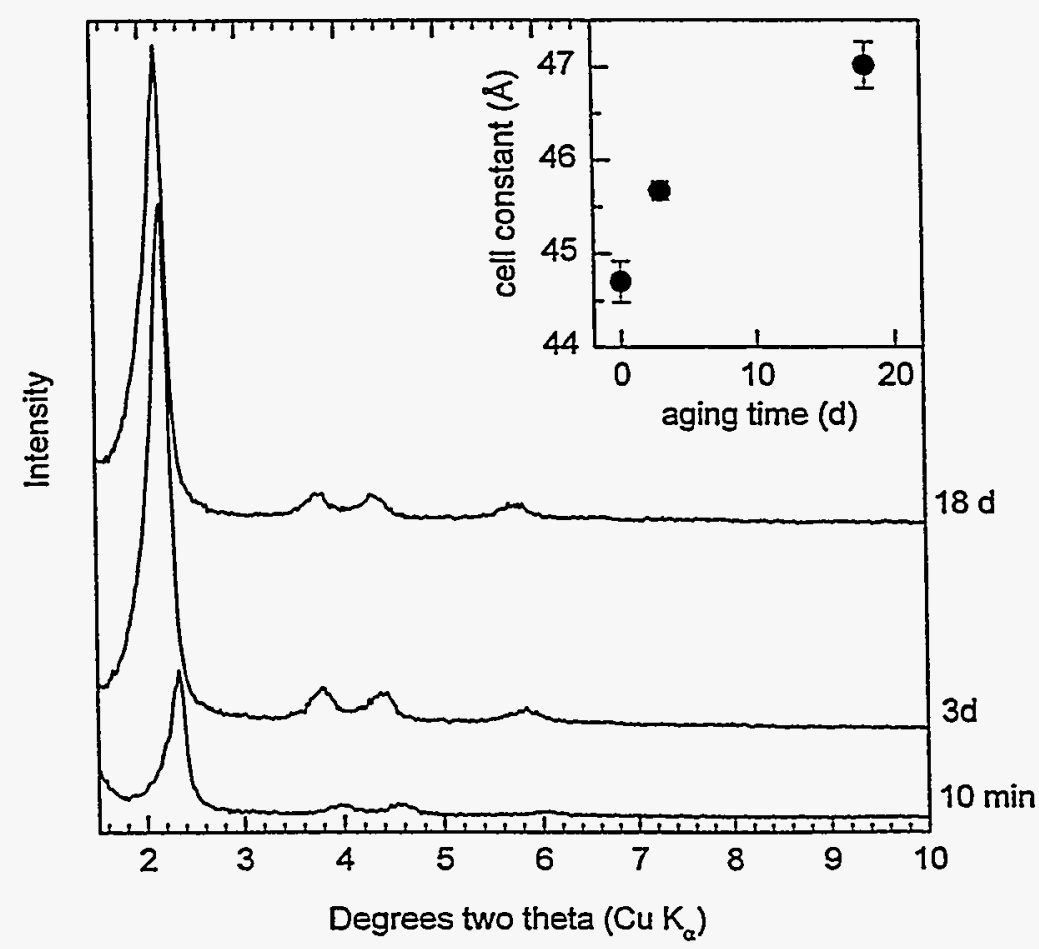

Figure 65. X-ray diffraction patterns for the water:methylene chloride system show that the cosolvent is slowly imibed into the hydrophobic interior of the surfactant arrays when aged at room temperature, which increases the cell constant. The regularity of the 2- $d$ array of pores is not affected by the long aging time. 
$12 \%$ for diethyl ether, $r \cong 2 \%$ for methylene chloride, and $r \cong 18 \%$ for THF, Figs. 60, 63, \& 64 .

The cause of the transition to the lamellar phase may be the tendency of the low dielectric constant solvent to partition inside of the hydrophobic tail region of the surfactant assemblies. Evidence for the slow incorporation of cosolvents is the increase in cell constants with aging time for the water:methylene chloride system, Fig. 65. The incorporation of cosolvents into the tail region increases the hydrophobic volume of the surfactant array, which has been shown to favor structures with lower curvature and head group volume (i.e. lamellar over hexagonal) [4, 47]. For example, in the THF system at $r=20 \%$ a hexagonal phase forms initially, Fig. 66, but over the course of several days the structure transforms to a lamellar product, Fig. 67, although the transformation is somewhat frustrated as evidenced by the residual hexagonal product. The transformation is thermally activated and can be driven to completion by aging the product at $60^{\circ} \mathrm{C}$, Fig 68 . The transformation occurs more rapidly the deeper into the lamellar region the initial value of $r$, but still appears to go through a hexagonal phase initially. For example, for $r=25 \%$ in the THF system there is already clear evidence that the transformation from $\mathrm{H}$ to $\mathrm{L}$ is occurring after only $10 \mathrm{~min}$, Fig. 69.

For cosolvents with intermediate dielectric constants (ethanol, isopropanol, acetone), $10<\varepsilon \leq 25$, the hexagonal region is larger, spanning $0 \%$ $\leq r<\sim 50 \%$, Figs. 70-72. In these cases it appears that a transformation from $\mathrm{H}$ to $\mathrm{D}$ occurs at high values of $r$ and that the $\mathrm{D}$ phase can persist at relatively high values of $r$. For example, for acetone there is a very weak peak at $r=60 \%$, Fig 72.

For polar cosolvents with large dielectric constants (methanol, ethylene glycol, acetonitrile, glycerol, tetraglyme, formamide, $\mathrm{N}$-methylformamide), $\varepsilon$ $>25$, the existence region is from $r=0 \%$ to $r>50 \%$, Figs. 73-79. The large ERHM region for these polar solvents is not particularly surprising, in view of the fact that liquid crystalline surfactant phases of CTAB have been 


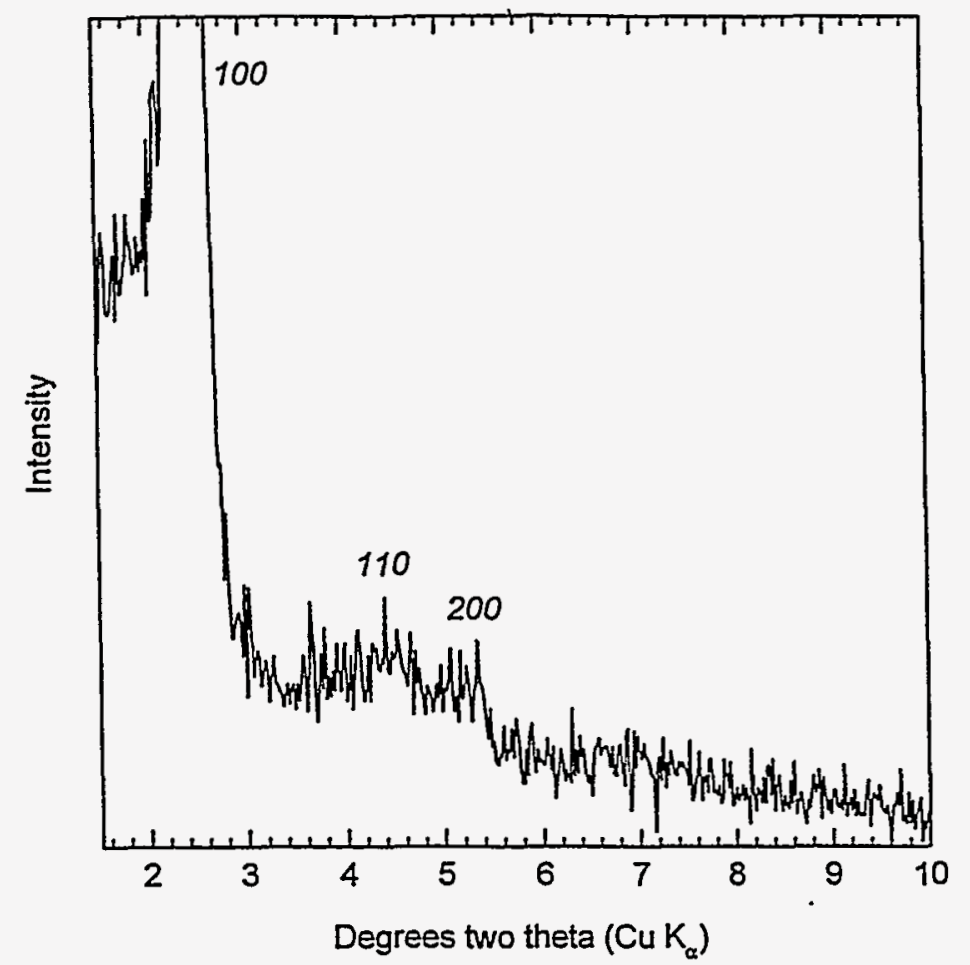

Figure 66. X-ray diffraction patterns for an $r=20 \%$ tetrahydrofuran sample aged $4 \mathrm{~h}$. The 110 and 200 reflections are evident, which, along with the 100 reflection, are definitive evidence for a hexagonal mesophase.

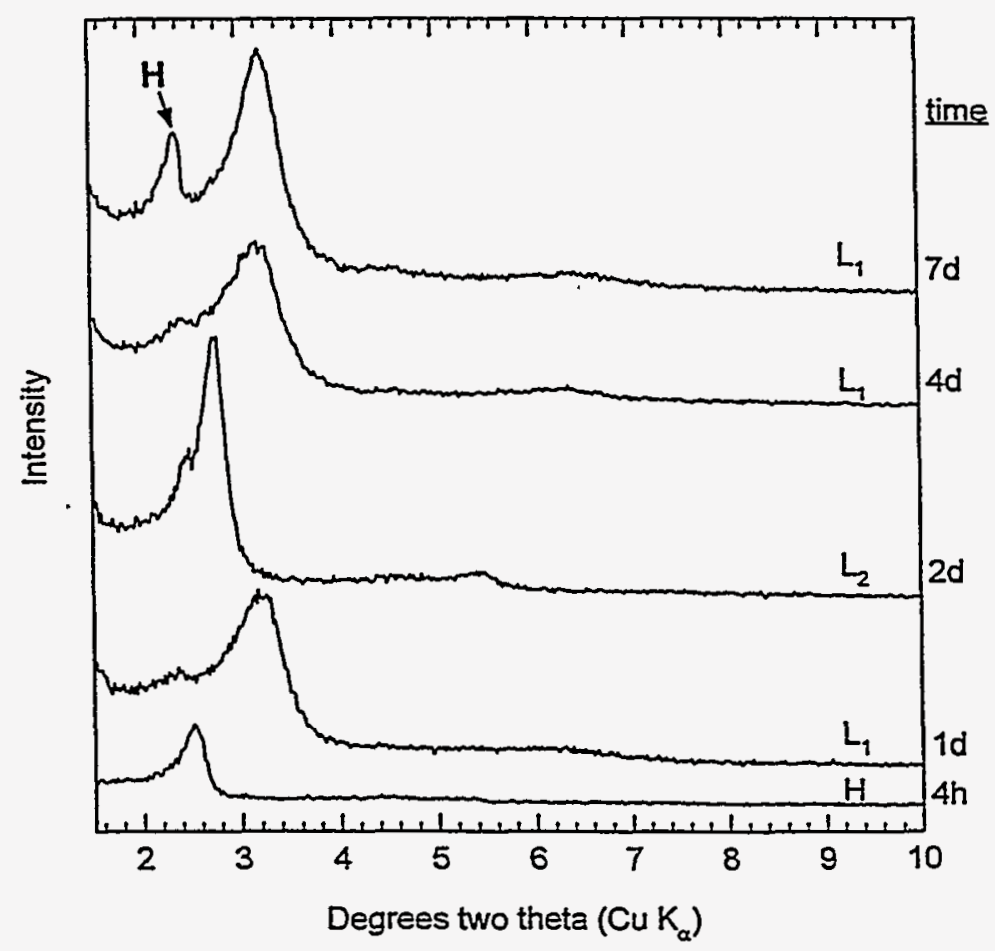

Figure 67. X-ray diffraction patterns for an $r=20 \%$ tetrahydrofuran sample aged at room temperature show the transformation from hexagonal $\left(\mathrm{H}_{\text {; }}\right.$ see Fig. 9) to lamellar $\left(\mathrm{L}_{1}\right)$ over the course of $1 \mathrm{~d}$. The $L_{1}$ phase transforms to an $L_{2}$ phase with a larger cell constant and then back to the $L_{1}$ phase. The transformation from $H$ to $L$ is not complete as evidenced by the persistent peak at $\sim 2.2$ to $2.5^{\circ}$ two theta. 


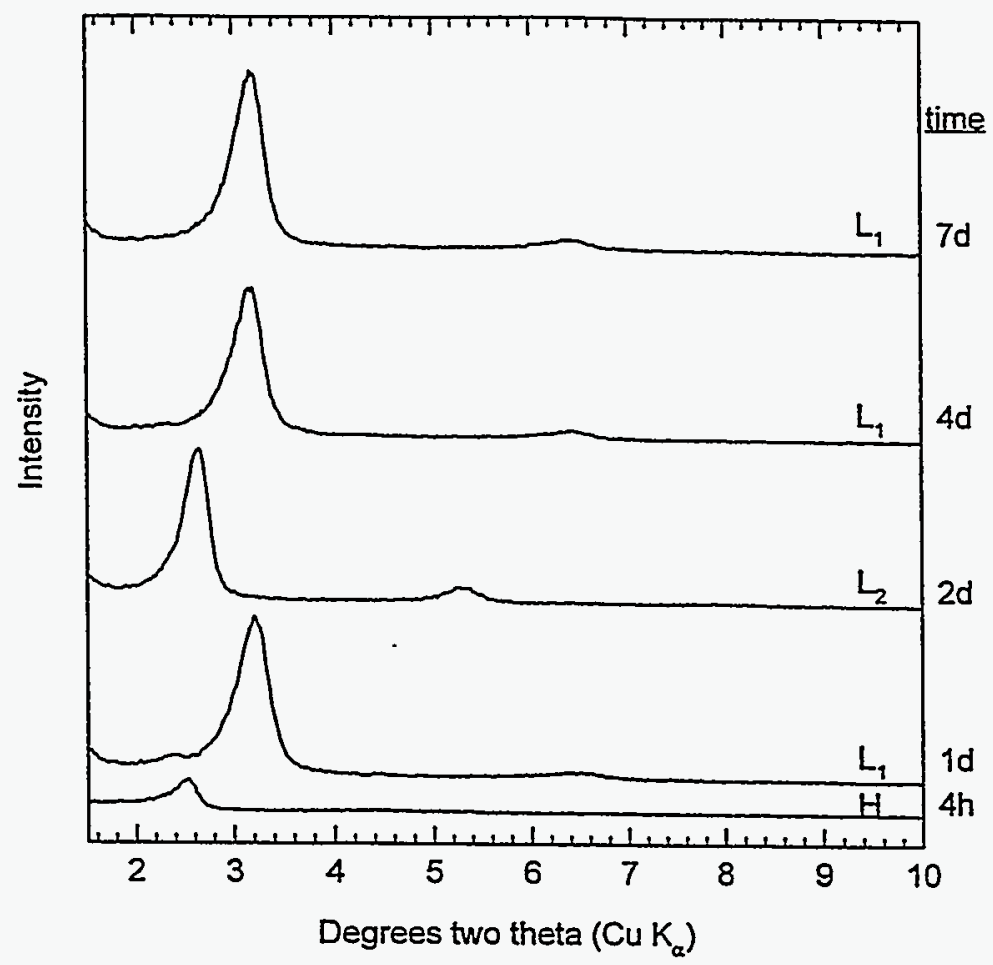

Figure 68. X-ray diffraction patterns the an $r=20 \%$ tetrahydrofuran sample aged at $60^{\circ} \mathrm{C}$ show the transformation from hexagonal $(\mathrm{H})$ to lamellar $\left(\mathrm{L}_{1}\right)$ over the course of $1 \mathrm{~d}$. The $\mathrm{L}_{1}$ phase transforms to an $L_{2}$ phase with a larger cell constant and then back to the $L_{1}$ phase. The transformation from $\mathrm{H}$ to $\mathrm{L}$ is not complete at this temperature.

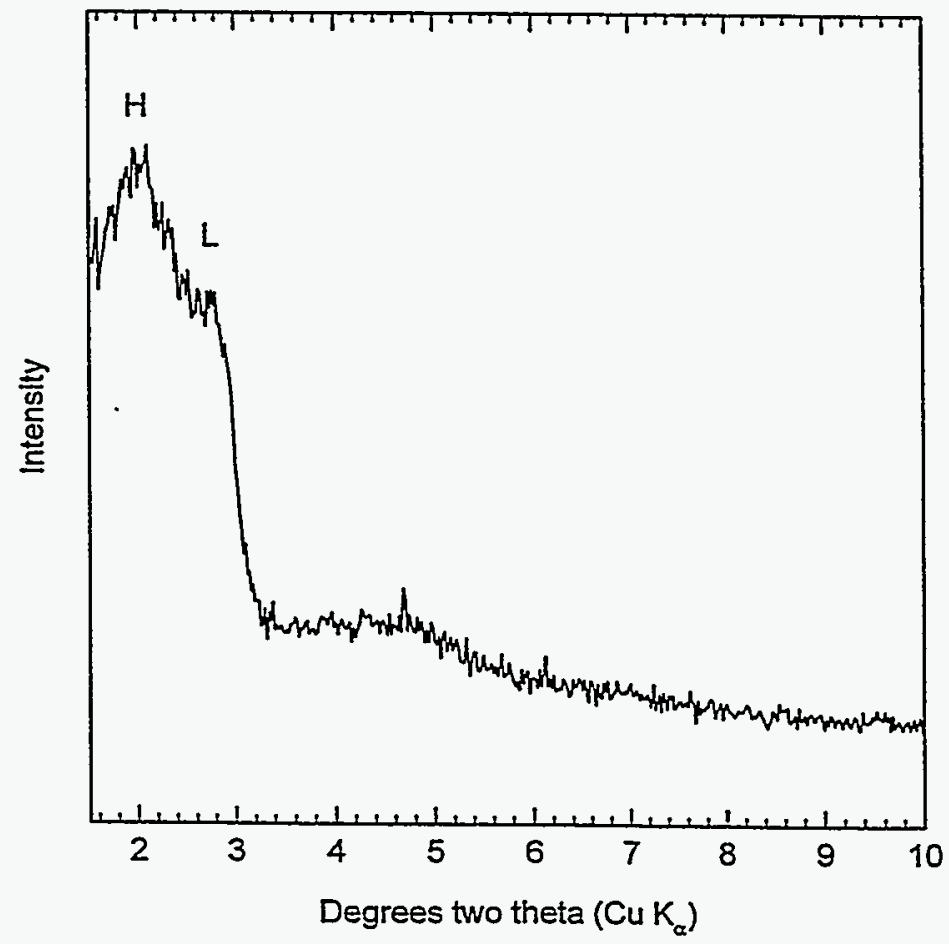

Figure 69. X-ray diffraction pattern of an $r=25 \%$ tetrahydrofuran sample aged for 10 minutes at room temperature. The sample contains peaks consistent with $\mathrm{H}$ and $\mathrm{I}$ phases, which implies the $\mathrm{H}$ to $\mathrm{L}$ transformation begins very shortly after the mesophase forms. 


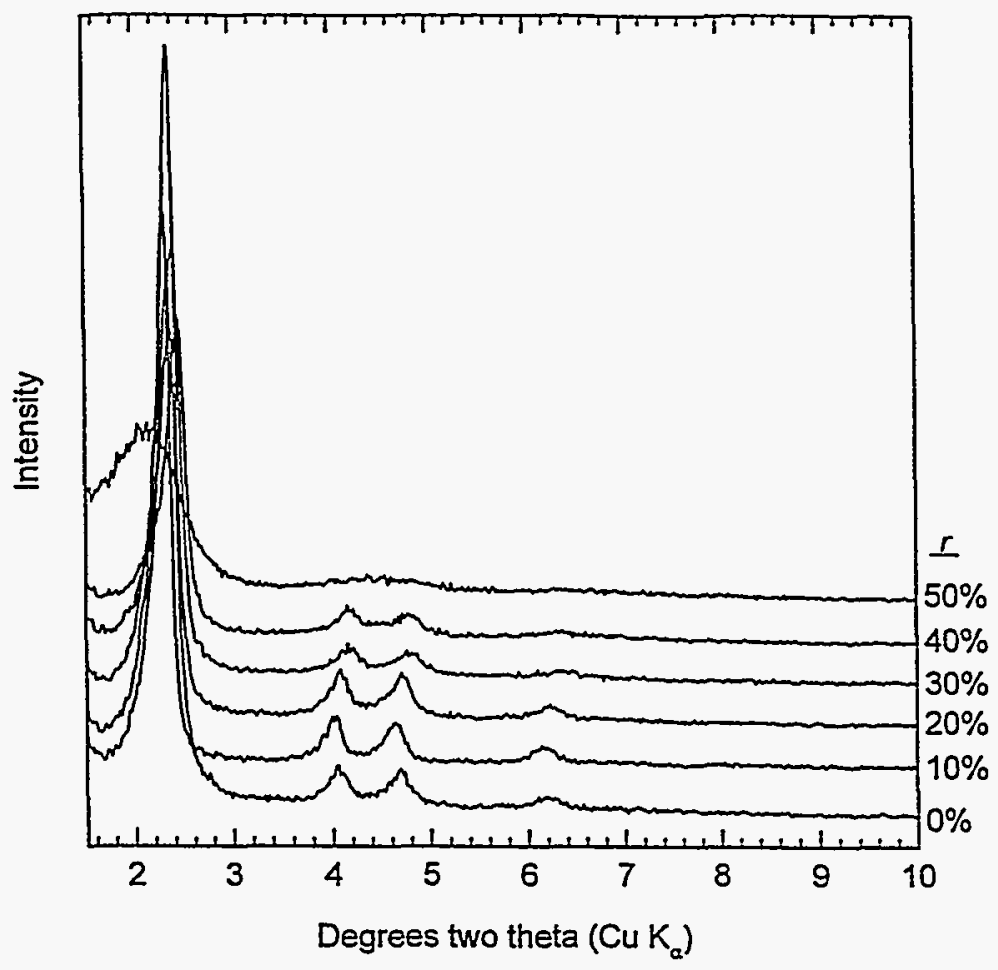

Figure 70. $X$-ray diffraction patterns for the water:ethanol system show the transformation from hexagonal to disordered at $r=40 \%$. Note the pronounced shift of the diffraction peaks to higher values of $2 \theta$ as $r$ increases, which implies the cell constant decreases as $r$ increases. The samples were aged 1 day.

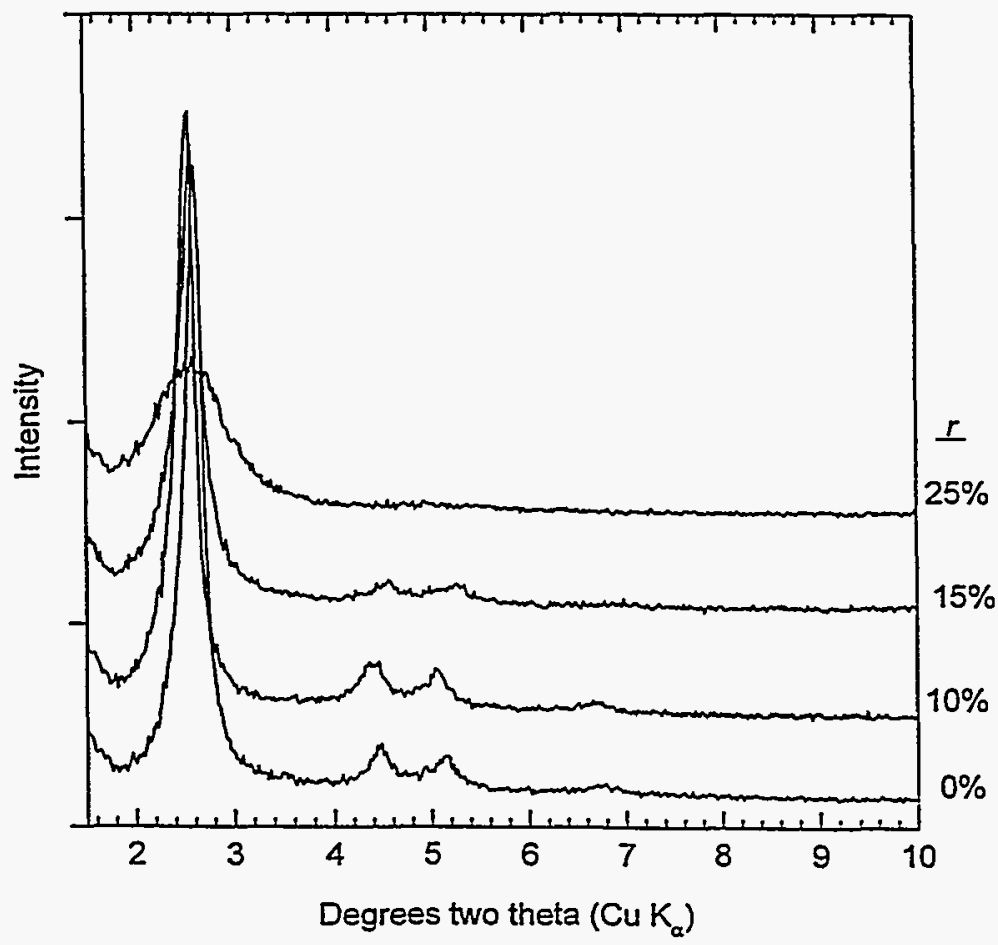

Figure 71. $X$-ray diffraction patterns for the water:isopropanol system show the transformation from hexagonal to disordered at $r=15 \%$. Note the pronounced shift of the diffraction peaks to higher values of $2 \theta$ as $r$ increases, which implies the cell constant decreases as $r$ increases. The samples were aged 5 days. 


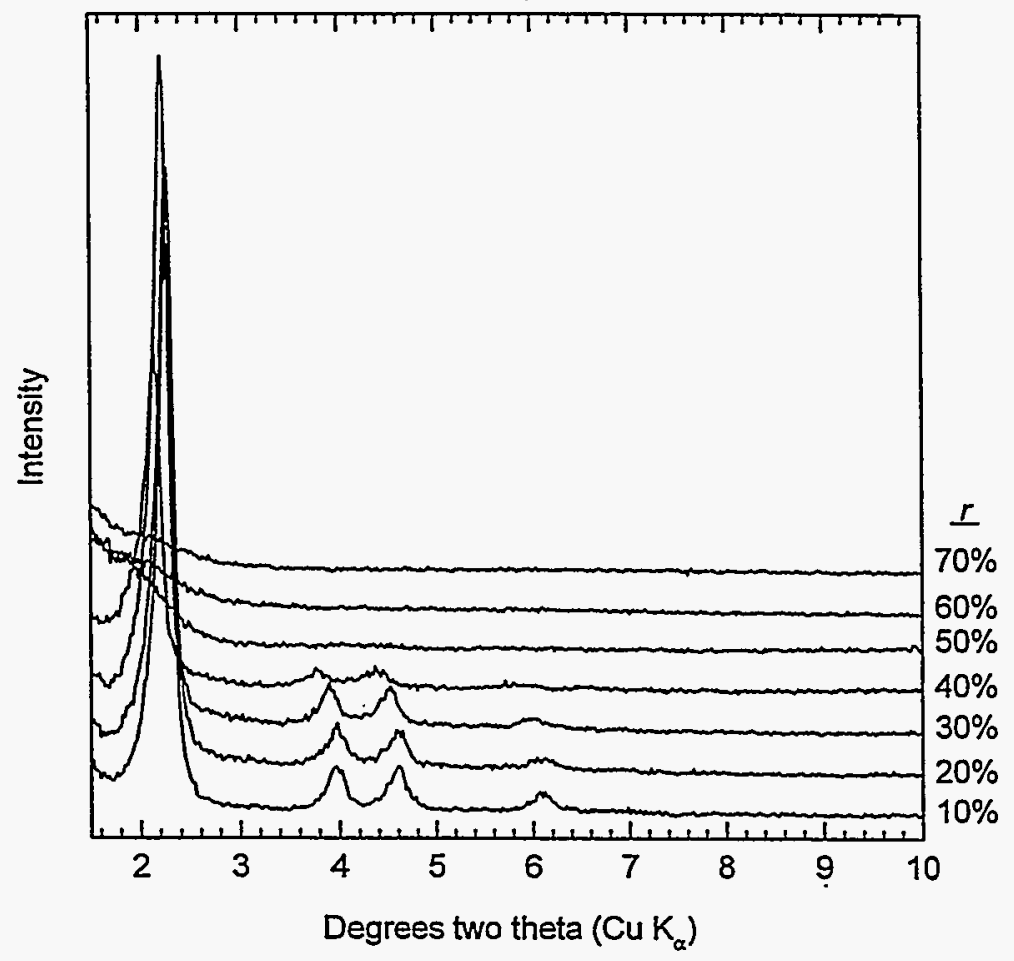

Figure 72. X-ray diffraction patterns for the water:acetone system show the transformation from hexagonal to disordered at $r \approx 40 \%$. Note the pronounced shift of the diffraction peaks to smaller values of $2 \theta$ as $r$ increases, which implies the cell constant increases as $r$ increases. The samples were aged 1 day.

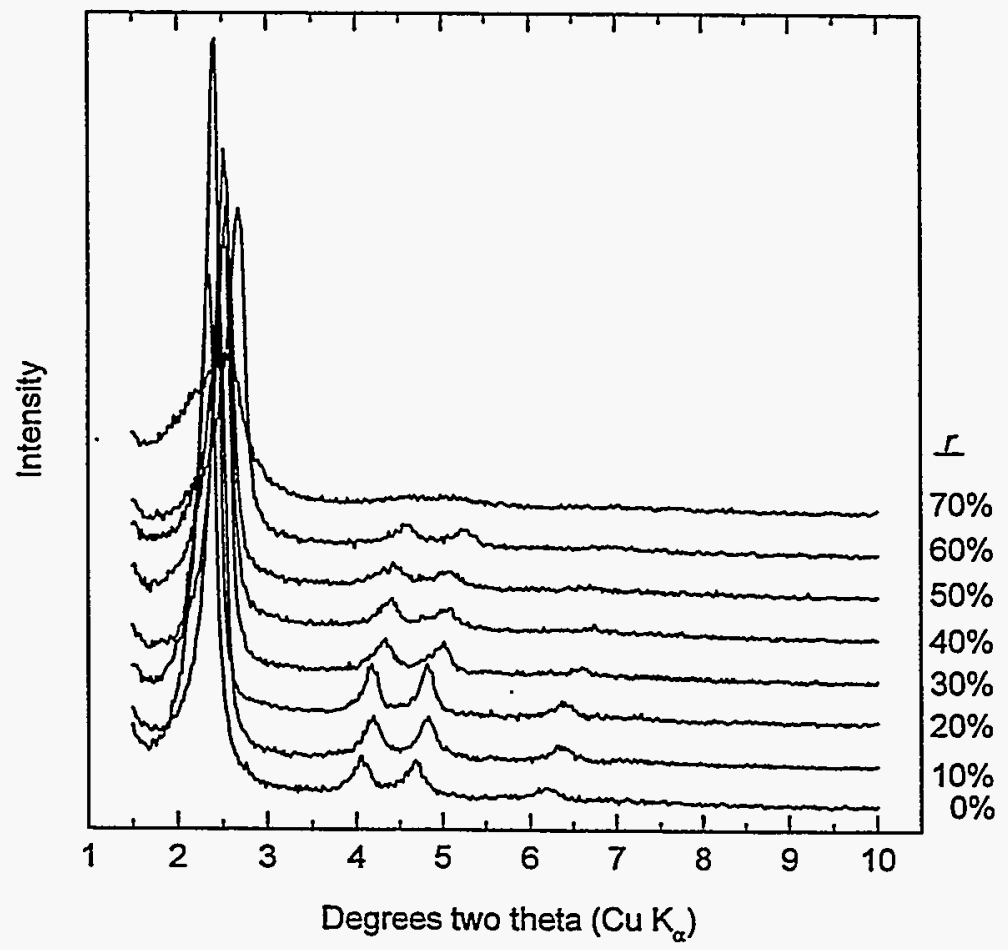

Figure 73. X-ray diffraction patterns for the water:methanol system show the transformation from hexagonal to disordered at $r \approx 60 \%$. Note the pronounced shift of the diffraction peaks to higher values of $2 \theta$ as $r$ increases, which implies the cell constant decreases as $r$ increases. The samples were aged 1 day. 


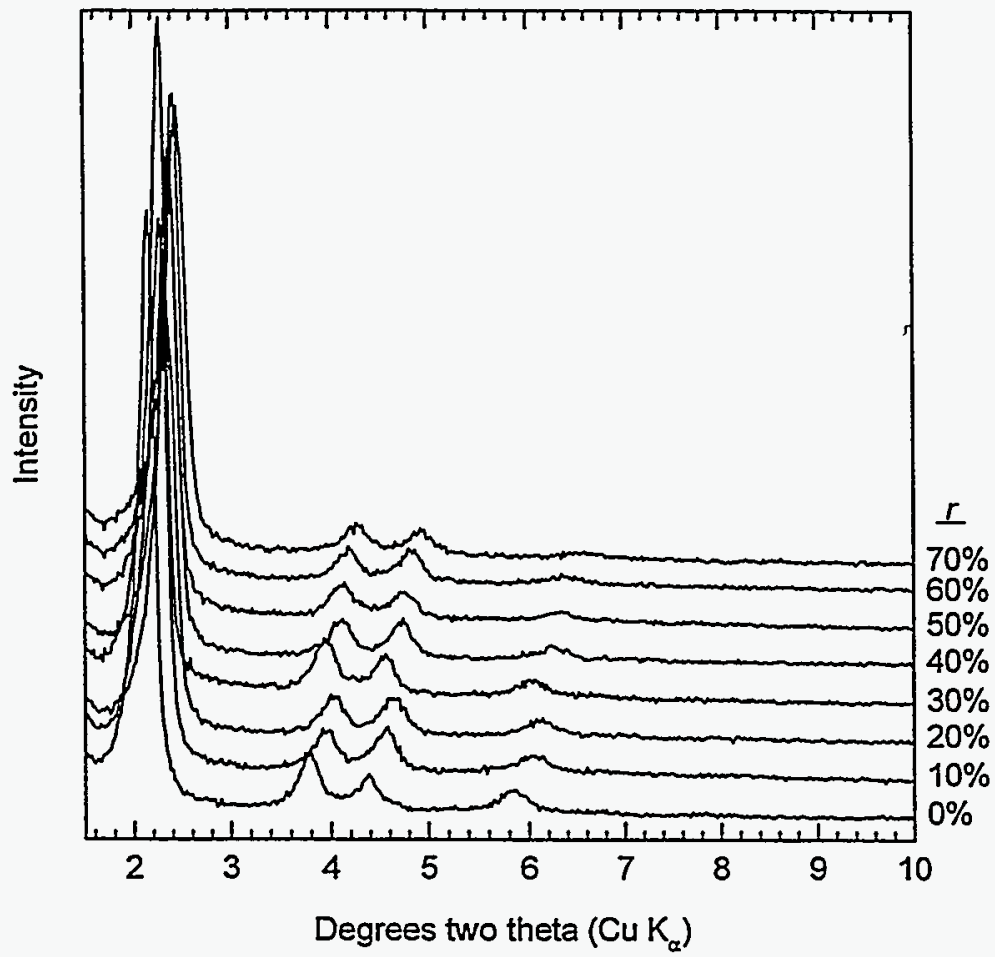

Figure 74. X-ray diffraction patterns for the water:ethylene glycol system show a pronounced shift of the diffraction peaks to higher values of $2 \theta$ as $r$ increases, which implies the cell constant decreases as $r$ increases. The ERHM is $>r=70 \%$. The samples were aged 1 day $(r=$ $10 \%$ to $50 \%)$ and 8 days $(r=60,70 \%)$.

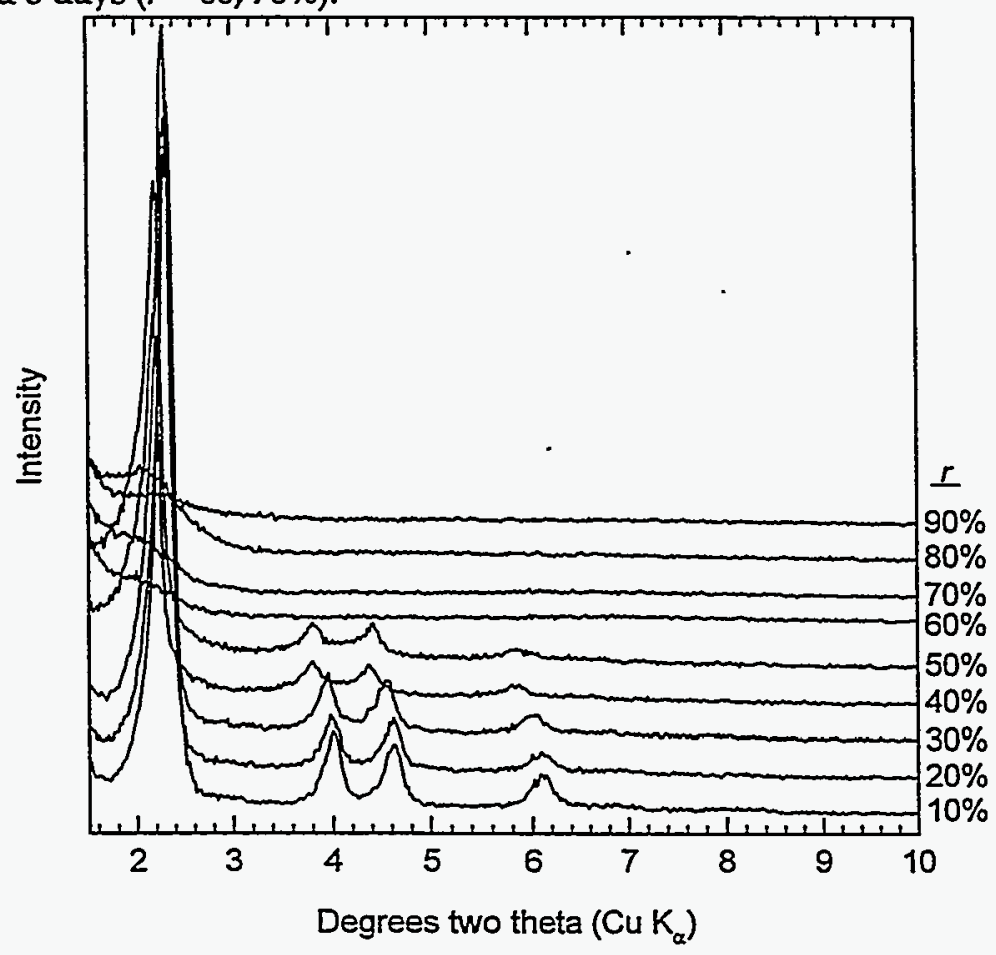

Figure 75. X-ray diffraction patterns for the water:acetonitrile system show the transformation from hexagonal to disordered at $r \approx 50 \%$. The solvent system phase separates at $50 \%$ at the high $\mathrm{pH}$ values used in these experiments. Note the shift of the diffraction peaks to lowere values of $2 \theta$ as $r$ increases, which implies the cell constant increases as $r$ increases. The samples were aged 2 days ( $r=0 \%$ to $50 \%$ ) and 5 days ( $r>50 \%$ ). 


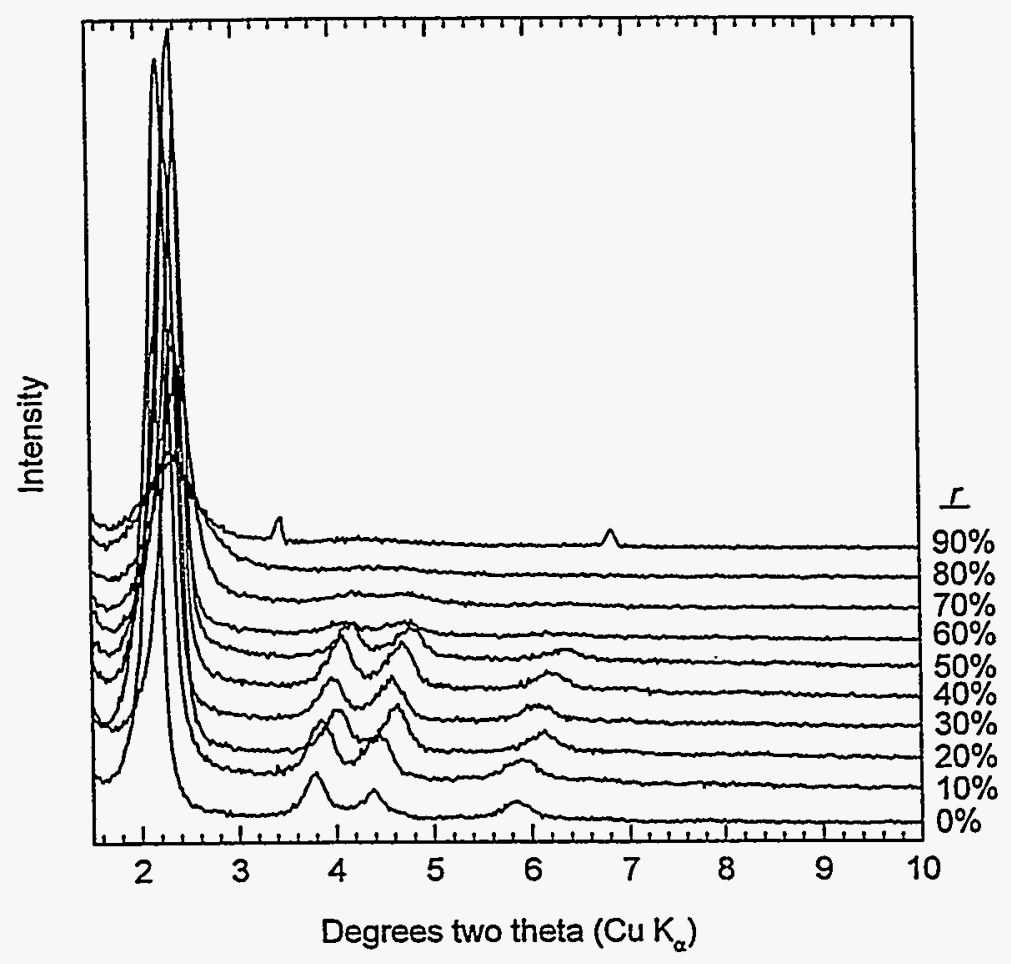

Figure 76. X-ray diffraction patterns for the water:glycerol system show the transformation from hexagonal to disordered at $r \approx 70 \%$. Note the pronounced shift of the diffraction peaks to higher values of $2 \theta$ as $\mathrm{r}$ increases, which implies the cell constant decreases as $r$ increases. The samples were aged 2 days $(r \leq 50 \%)$ and 1 day $(r>50 \%)$.

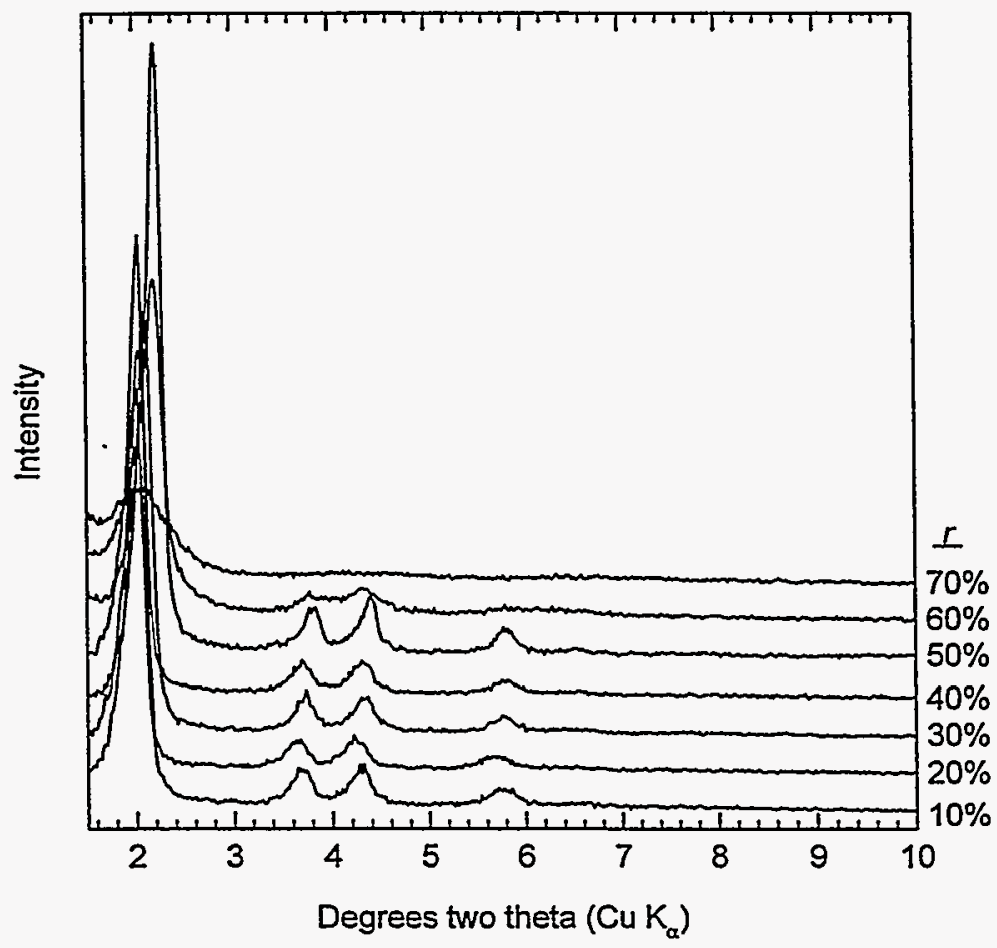

Figure 77. X-ray diffraction patterns for the water:tetragiyme system show the transformation from hexagonal to disordered at $r \approx 70 \%$. The diffraction peaks shift to higher values of $2 \theta$ as $r$ increases, which implies the cell constant increases as $r$ increases. The samples were aged 5 days. 


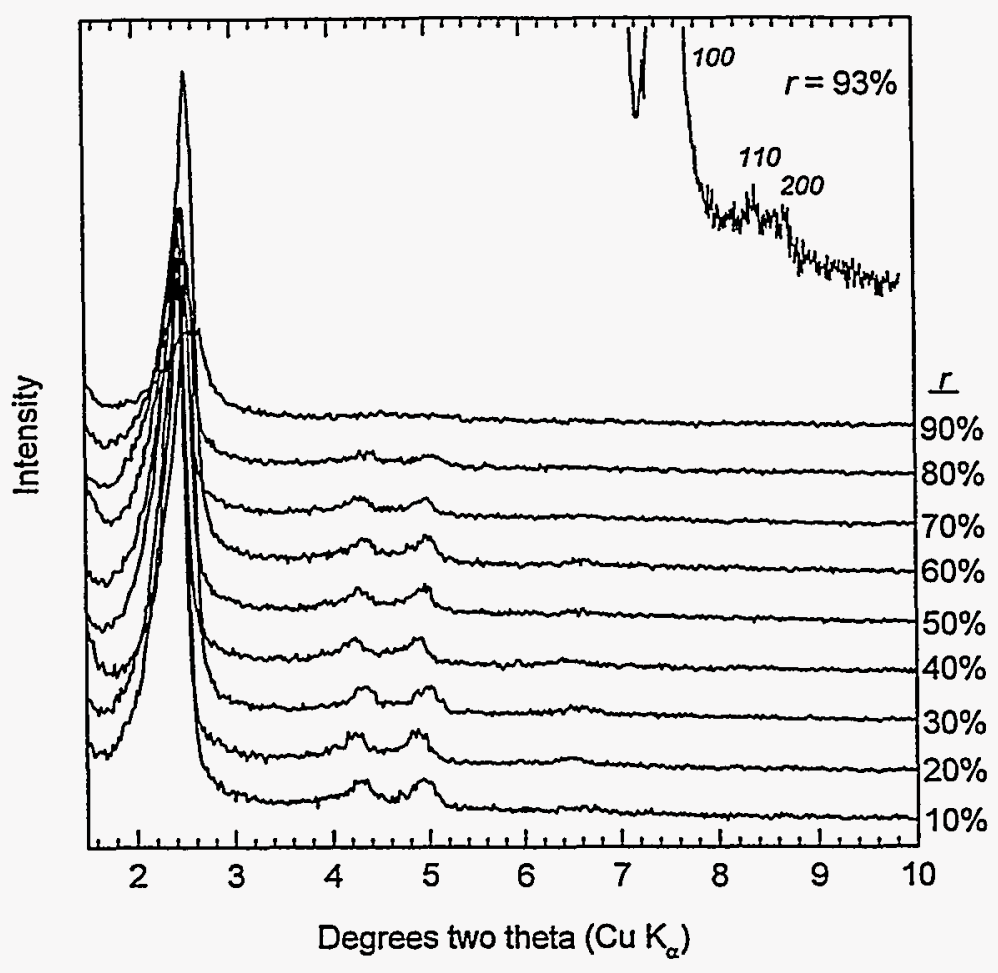

Figure 78. X-ray diffraction patterns for the water:formamide system show the transformation from hexagonal to disordered at $r \approx 93 \%$. Note the pronounced shift of the diffraction peaks to higher values of $2 \theta$ as $r$ increases, which implies the cell constant decreases as $r$ increases.

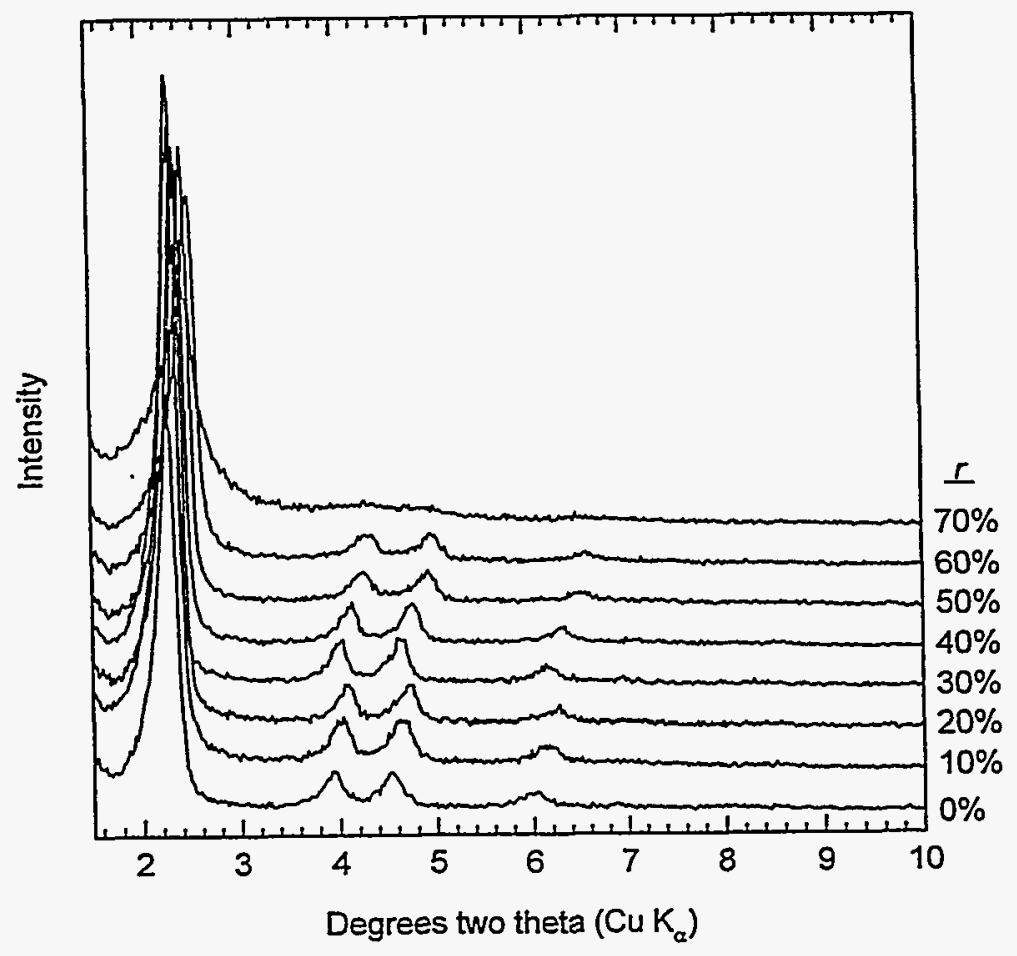

Figure 79. X-ray diffraction patterns for the water:N-methylformamide system show the transformation from hexagonal to disordered at $r \approx 60 \%$. Note the pronounced shift of the diffraction peaks to higher values of $2 \theta$ as $r$ increases, which implies the cell constant decreases as $r$ increases. The samples were aged 1 day. 
reported for the protic donor solvents glycerol, ethylene glycol, formamide, and N-methylformamide [48,49].

We also examined nine mixed-cosolvent systems by combining formamide with tetraglyme in three ratios $(25: 75,50: 50$, and $75: 25)$ ratios and adding these solutions to water with three water to mixed-cosolvent ratios ( $r$ $=70,80$, and 90\%). We found that the ERHM in the 75:25 formamide:tetraglyme system is $r \geq 90 \%$, Fig. 80, and is $r=80 \%$ in the $50: 50$ and 25:75 systems, Figs. $81 \& 82$.

D.2 Nonaqueous Synthesis - One goal of this research is to develop a nonaqueous route to hexagonal mesoporous silica. We would call strictly nonaqueous a synthetic route wherein the water:TMOS molar ratio $(h)$ is less than or equal to 4 , and the diffraction data show at least three peaks. We would term substantially nonaqueous any method that approaches this ideal.

We have seen that for glycerol and methanol $D$ mesophase products can be made with $r$ equal to at least $90 \%$, corresponding to $h=12.9$. With formamide $\mathrm{H}$ products can be made with $r=93 \%$, corresponding to $h \cong 9.0$. Formamide will form $\mathrm{D}$ products at $\mathrm{r}=96 \%(h=5.2$, diffraction peak at $d \approx 33$ $\AA)$, Fig. 83. With ethylene gycol D products actually form with $h<4(r=97 \%)$, as shown in Fig. 84. We would call these syntheses with formamide and ethylene glycol substantially nonaqueous.

At sufficiently high values of $r$ that $D$ phases no longer form transparent chemical gels form over several hours in these four polar solvents. The surfactant apparently no longer acts as a chemical dipole and/or there is simply not enough water to fully hydrolyze the silicate precursors that then drive the surfactant to a liquid crystalline state.

It is still an issue as to whether the samples synthesized in a substantially nonaqueous environment are sufficiently robust to survive calcination. Fig. 85 shows that a sample made with $r=90 \%$ formamide is indeed stable to calcination at $550^{\circ} \mathrm{C}$. Fig. 86 shows the isotherm for the 


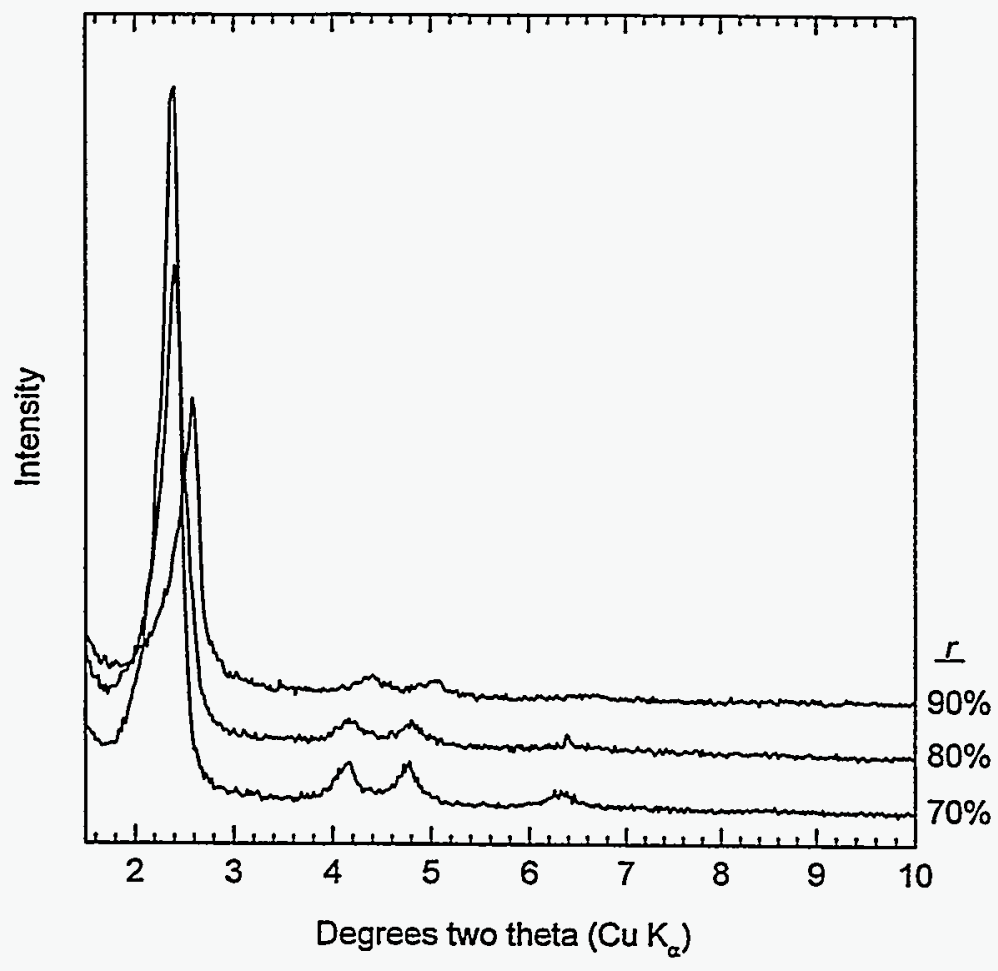

Figure 80. X-ray diffraction patterns for the water:(75:25 formamide:tetraglyme) system show that $\mathrm{H}$ products can be made at $r=90 \%$. The samples were aged 4 days.

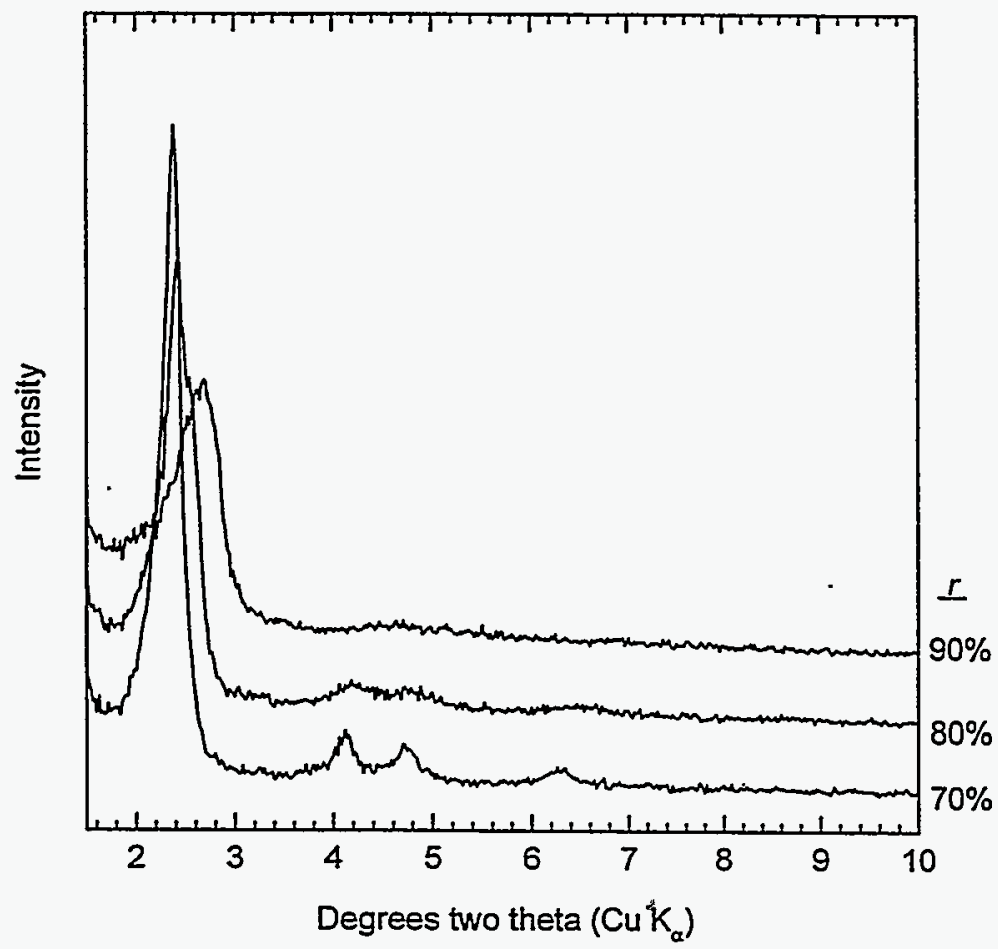

Figure 81. X-ray diffraction patterns for the water:(50:50 formamide:tetraglyme) system show that $\mathrm{H}$ products can be made at $r=80 \%$. The samples were aged 4 days. 


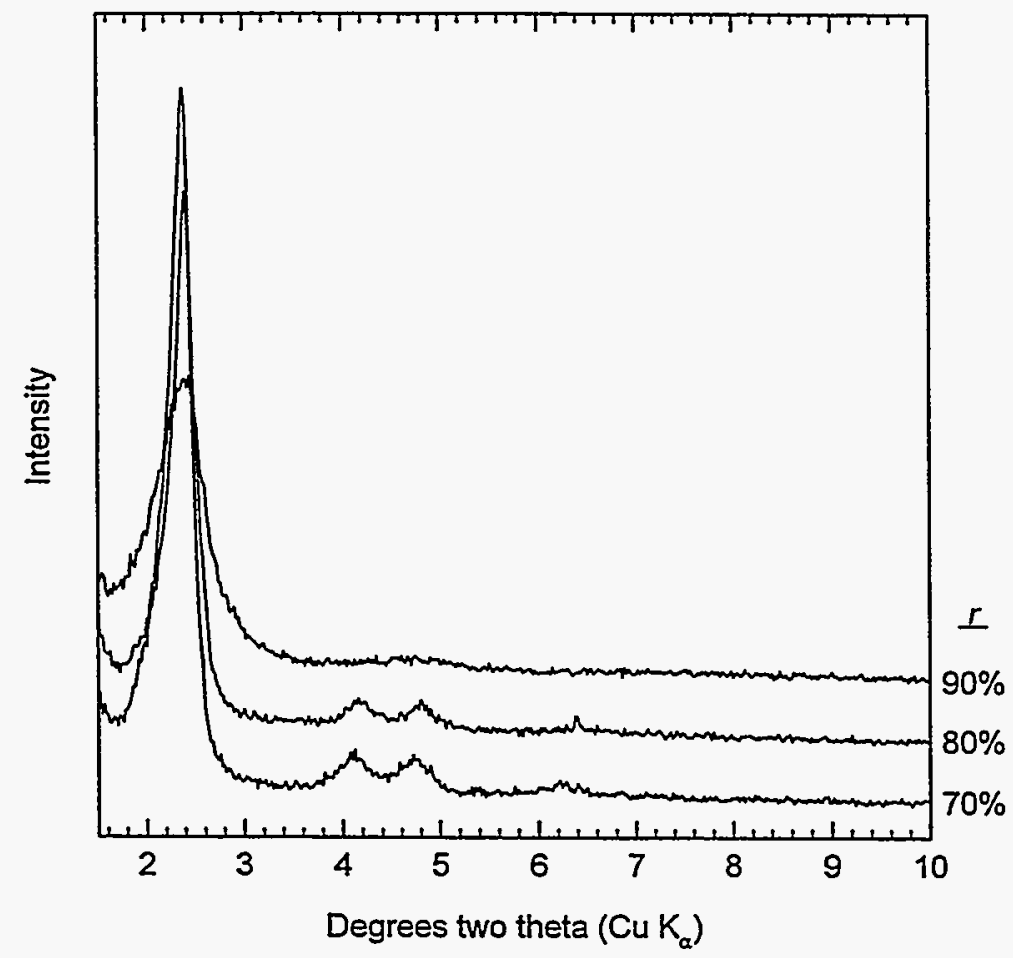

Figure 82. $X$-ray diffraction patterns for the water:(25:75 formamide:tetraglyme) system show that $\mathrm{H}$ products can be made at $r=80 \%$. The samples were aged 4 days.

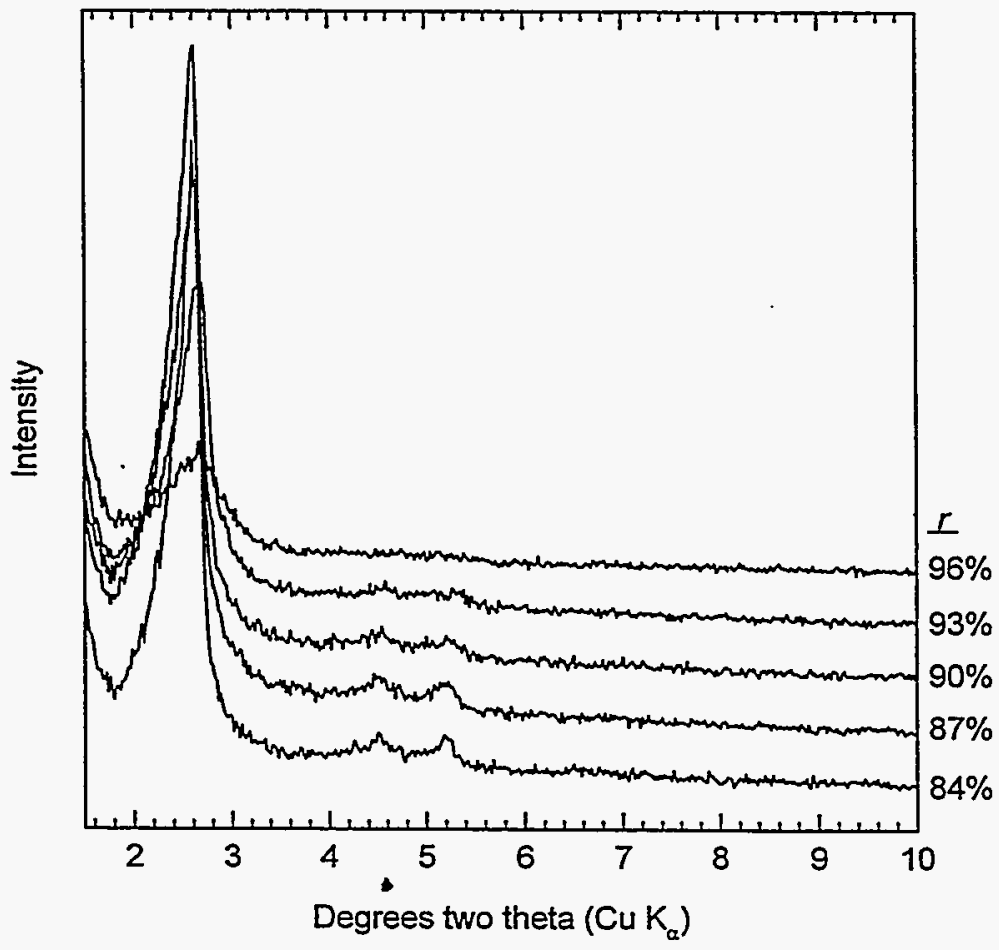

Figure 83. X-ray diffraction pattern for the water:formamide system at high values of $\mathrm{r}$ show that disordered products can be made up to $r=96 \%$. At $r=99 \%$ a clear transparent amorphous silica chemical gel forms. 
calcined sample from which we determined the sample has a high surface area and narrow pore size distribution.

It is useful to consider why the some high dielectric constant cosolvents, specifically methanol, acetonitrile, tetraglyme, and $\mathrm{N}$ methylformamide, do not support mesophase formation in substantially nonaqueous environments. For example, with methanol at $r=95 \%$ a transparent chemical gel forms. In this case the cmc, as determined by light scattering, exceeds the $2 \%$ surfactant concentration when $r$ » $60 \%$. Apparently, at methanol concentrations the surfactant is no longer an effective chemical dipole.

The cosolvent acetonitrile has limited miscibility, phase separating at $r$ » 50\%, and limiting $h$ to $\sim 65$.

Tetraglyme is aprotic, which prevents strong intermolecular hydrogen bonding. This may cause the reduced $\mathrm{cmc}$ and the more limited range over which the surfactant acts as a chemical dipole.

The dielectric constant of $\mathrm{N}$-methylformamide in anomalously high. The effective charge screening provided by the solvent may actually destabilize the inorganic-organic salt formed in mesophases, and may thus frustrate the transformation from surfactant plus silica to an ordered liquid crystalline mesophase. On the other hand, added salts also screen charge and yet lead to the hexagonal product.

D.3 Cosolvents, Micellization and Product Periodicity - We have seen that we can easily make the hexagonal product with a variety of cosolvents, but that the maximum concentration that can be used depends on the dielectric constant etc. We felt that the trend could be related to the extent of micellization in the precursor solutions, which we can determine from static light scattering measurements. In essence, the formation of micelles indicate that the surfactant is acting as a chemical dipole, which is probably necessary to the formation of a periodic product. We have previously shown that this is the case in the water/methanol system. 


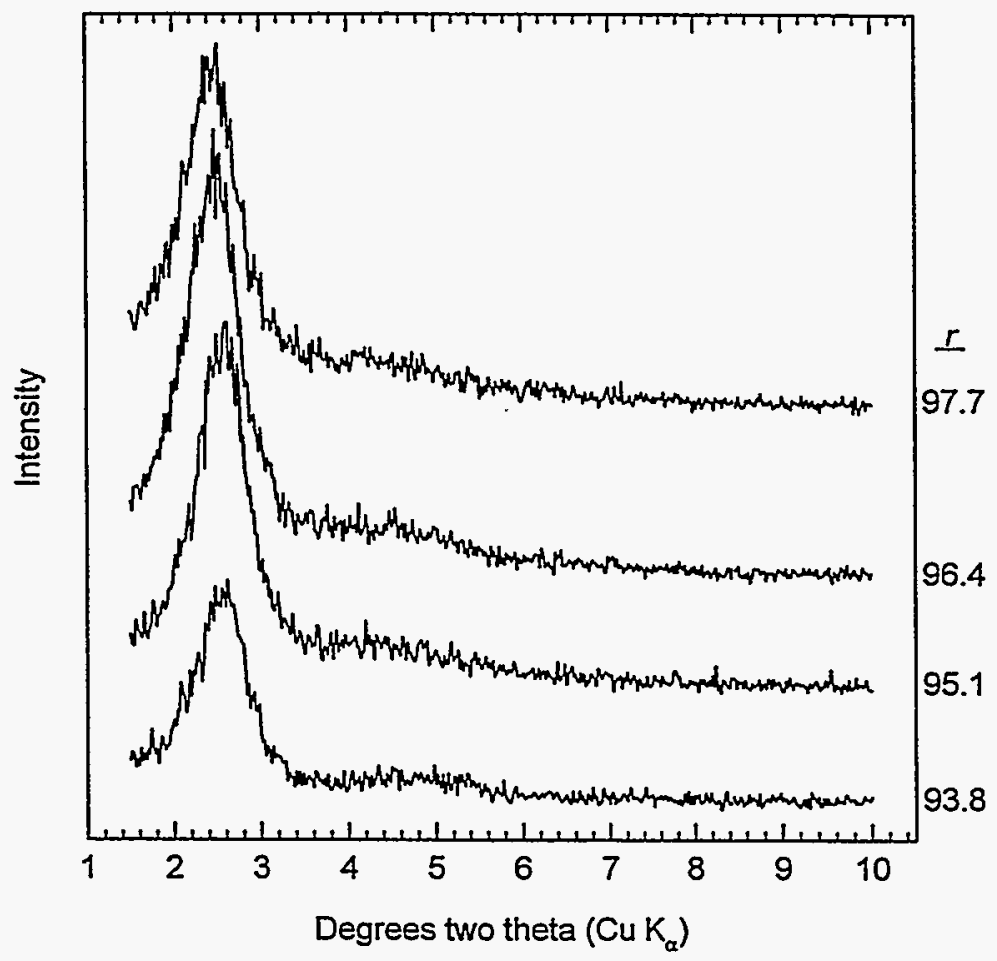

Figure 84. X-ray diffraction pattern for the water:ethylene glycol system at high values of $\mathrm{r}$ show that disordered products can be made up to $r=97.7 \%$.

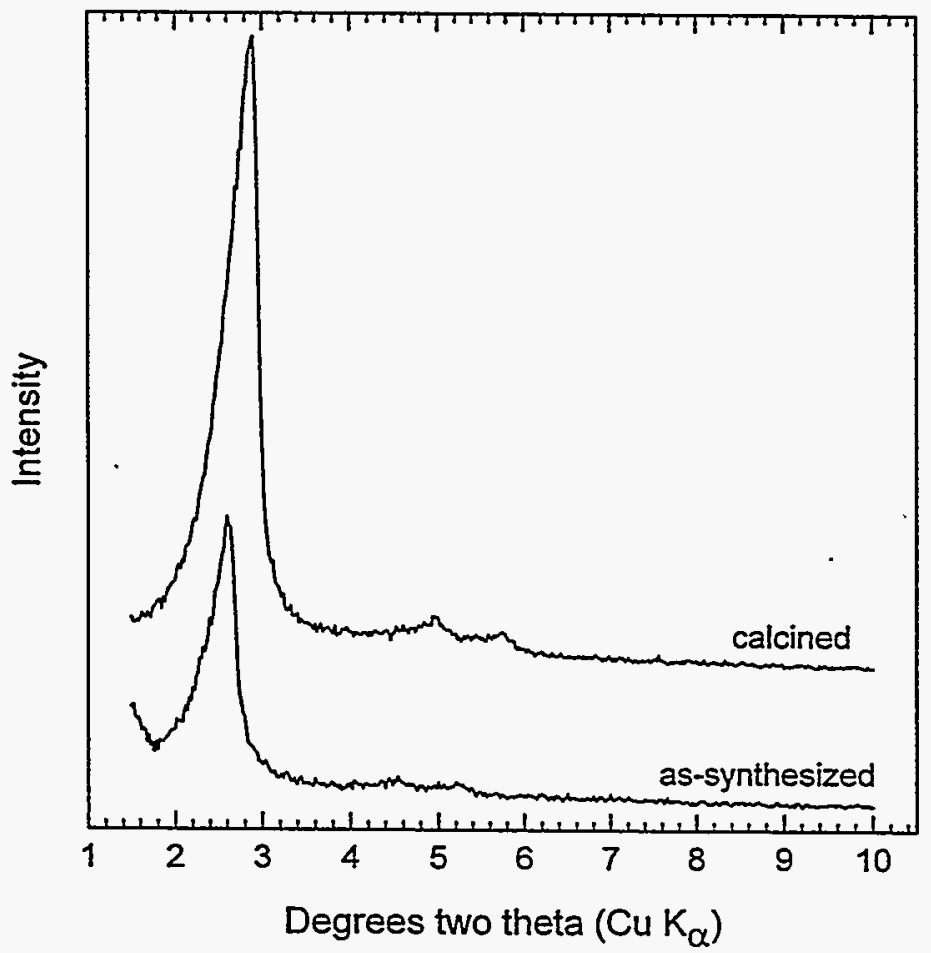

Figure 85. $X$-ray diffraction patterns of the an as-made and calcined $r=90 \%$ formamide sample. The calcined sample exhibits three peaks, which indicates it retains its periodic hexagonal arrangement of pores. The cell constant shrinks $\sim 8.5 \%$ owing primarily to the condensation of framework silanol groups at high temperature. 


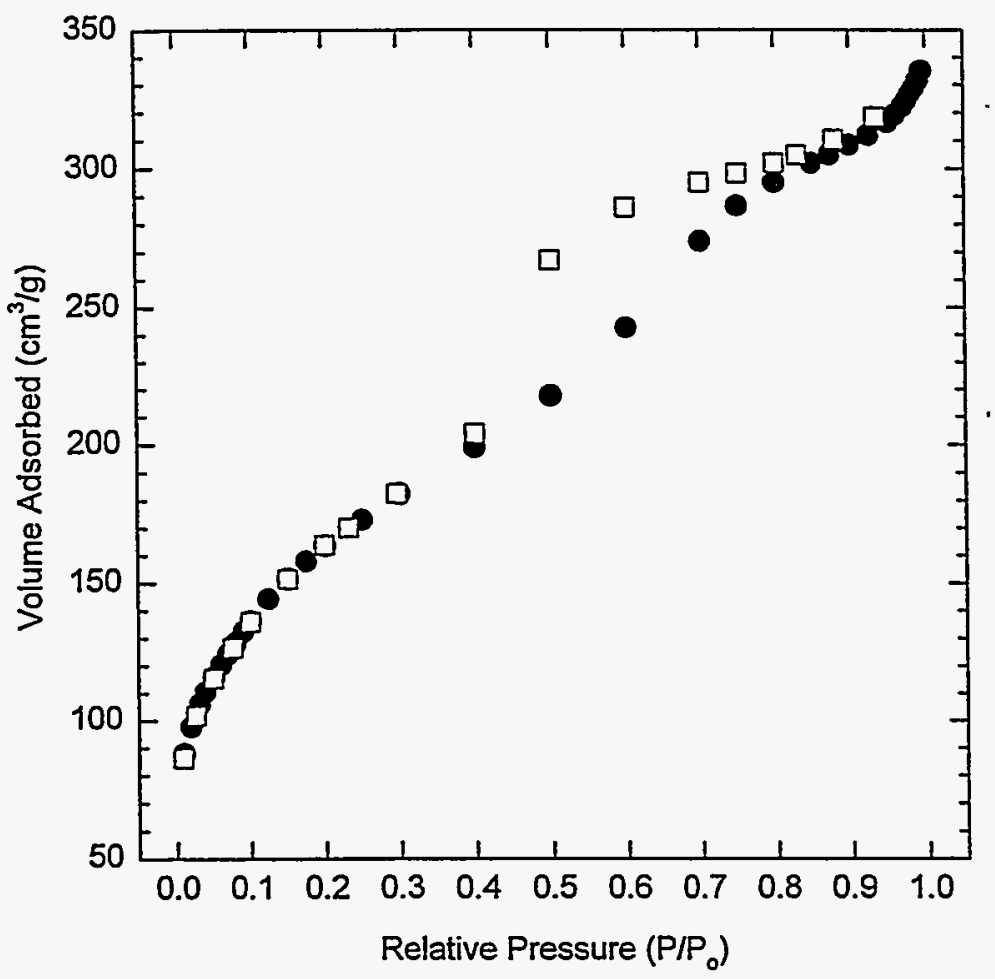

Figure 86. $\mathrm{N}_{2}$ sorption isotherm collected at $77 \mathrm{~K}$ on the $r=90 \%$-formamide sample shown in Fig. 27. The surface area is $550 \mathrm{~m}^{2} / \mathrm{g}$ and the pore volume is $0.52 \mathrm{cc} / \mathrm{g}$. A BJH analysis of the desorption branch shows that the maximum in $d V / d r$ is at 32-35 $\AA$.

We found that many of the cosolvents used here decrease the extent of micellization as $r$ increases in a $2 \mathrm{wt} \% \mathrm{CTAB}, \mathrm{pH}=13.2 \mathrm{NaOH}$, water:cosolvent solution Fig. 87. (In fact we assume this is the case for all cosolvents, but have not directly measured the change in scattered light intensity for all of them.)

The decreased micellization in the precursor solutions is accompanied by a steady but gradual decrease in the coherent scattering domain size (CSD), as determined from applying the Scherrer equation to XRD data, as $c^{*}$ decreases to zero $\left(c^{*}=c-c m c\right)$. For $c^{*}$ less than zero the CSD dramatically decreases, with an apparent break at $c^{*} \approx 0$ (compare the maximum value of $r$, Fig. 59 with the value of $r$ at $c^{*}=0$ in Fig. 87). Thus micellization of the surfactant in the precursor solutions is a good indicator of long-range 2-d hexagonal order: solutions that contain no micelles lead to $\mathrm{D}$ or A products, whereas solutions that contain micelles usually lead to $H$ products. Finally, 
the ability to form periodic mesophases with $c^{*}<0$ shows that the system can actually organize from free surfactant and molecular silicate precursors!

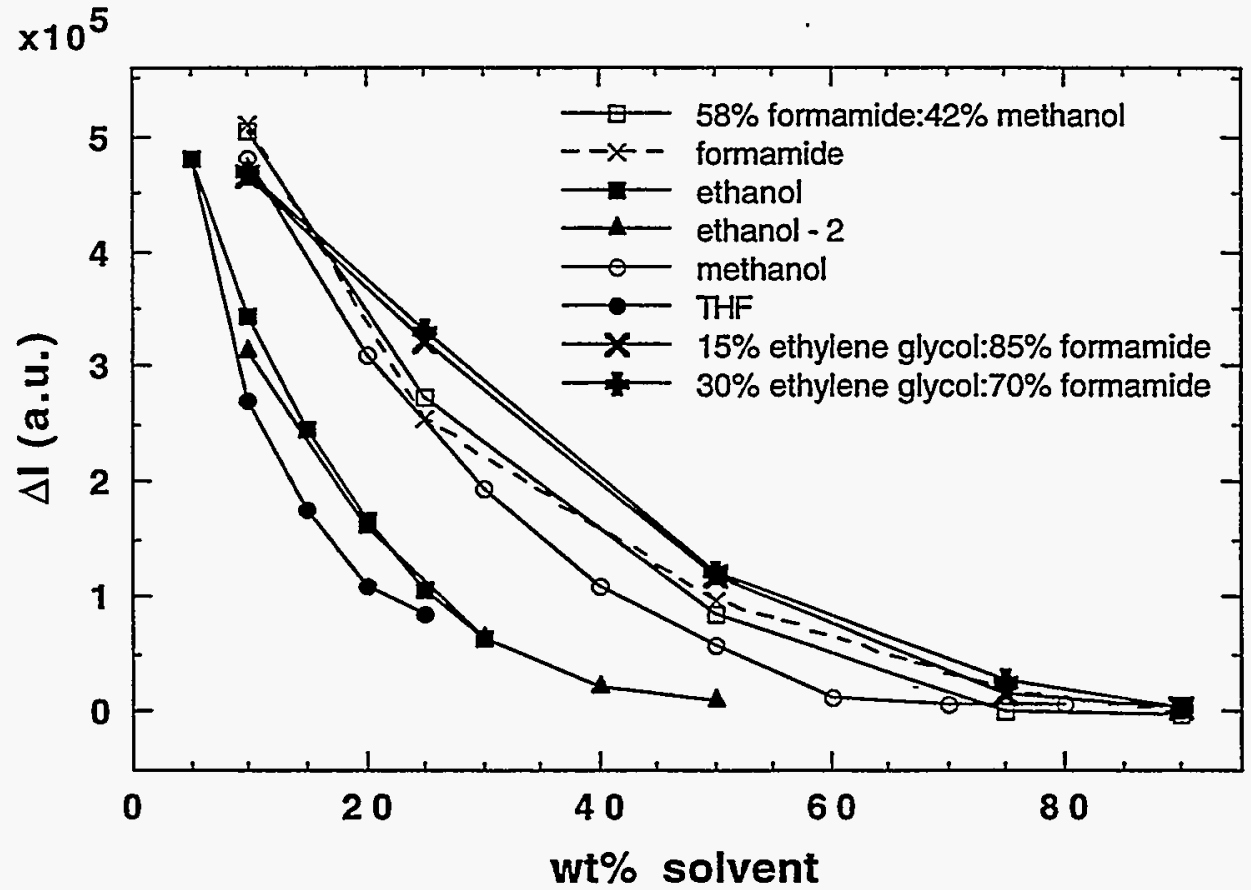

Figure 87. Static light scattering show that the intensity above background varies widely for a variety of cosolvents. The cmc » $0 \%$ where the differential scattered intensity is zero. Note that the cmc increases as the dielectric constant of the cosolvent increases.

D.4 Pore Size Control - In addition to regulating the water concentration and order parameter, cosolvents can be used to continuously tune the pore diameter of the mesoporous products. That this can occur is reasonable in light of the fact that it is well known that cosolvents can affect the aggregation number and radius of micelles [47]. We therefore set out to determine to what extent the micelle size determines the pore size.

Dynamic light scattering of the model system water:methanol demonstrated that the cosolvent decreases the micelle hydrodynamic radius as $r$ increases, Fig. 88 [33]. XRD data demonstrated an incommensurate decrease in the mesophase lattice parameter as $r$ increased. This demonstrates 
that the coupling is not simple, and that the micelles are protean structures greatly affected by the hydrolysis of TMOS.

Further studies show that cosolvents can either increase or decrease the lattice spacing. Protic cosolvents that can hydrogen bond, such as methanol, decrease the lattice spacing, whereas aprotic cosolvents increase the lattice spacing, Fig 89.

The cosolvent-solvent and cosolvent-surfactant interactions are undoubtedly complex, but the different effects of protic versus aprotic cosolvents may lie in their locus of solubilization within a micelle. Protic cosolvents are believed to be solubilized nearer the headgroups of the surfactant molecules - in proximity to the polar solvent [47]. Protic solvents thus have the ability to reduce repulsive headgroup interactions and reduce micelle diameter. Aprotic cosolvents are believed to be solubilized deeper within the micelle where they tend to expand the hydrophobic volume and increase the micelle size [47]. Regardless of the microscopic nature of the effect, cosolvents can be used to continuously tune the unit cell constant (and pore diameter)[33] of the mesophases.

D.5 Mixed-Metal Frameworks - Another advantage of the cosolvent approach to mesoporous oxides is that two or more alkoxides can be mixed and prehydrolyzed in an alcoholic environment, which can - by matching hydrolysis rates of the alkoxides - lead to a precursor solution that contains heteroatomic $\mathrm{M}-\mathrm{O}-\mathrm{M}^{\prime}$ bonds. This precursor solution can be used to synthesize a mesophase product with a homogeneous distribution of dopant ions in the framework. (A similar approach was used by Kim et al. [50], who used a $10 \% \mathrm{CTACl}$ in formamide solution to dissolve titanium n-butoxide and then added precipitated silica to this followed by aqueous sources of dissolved silica. In their case mesophases were formed from $r=86 \%, h=8.6$, $[\mathrm{Si}] \approx 0.92 \mathrm{M}$ solutions.) 


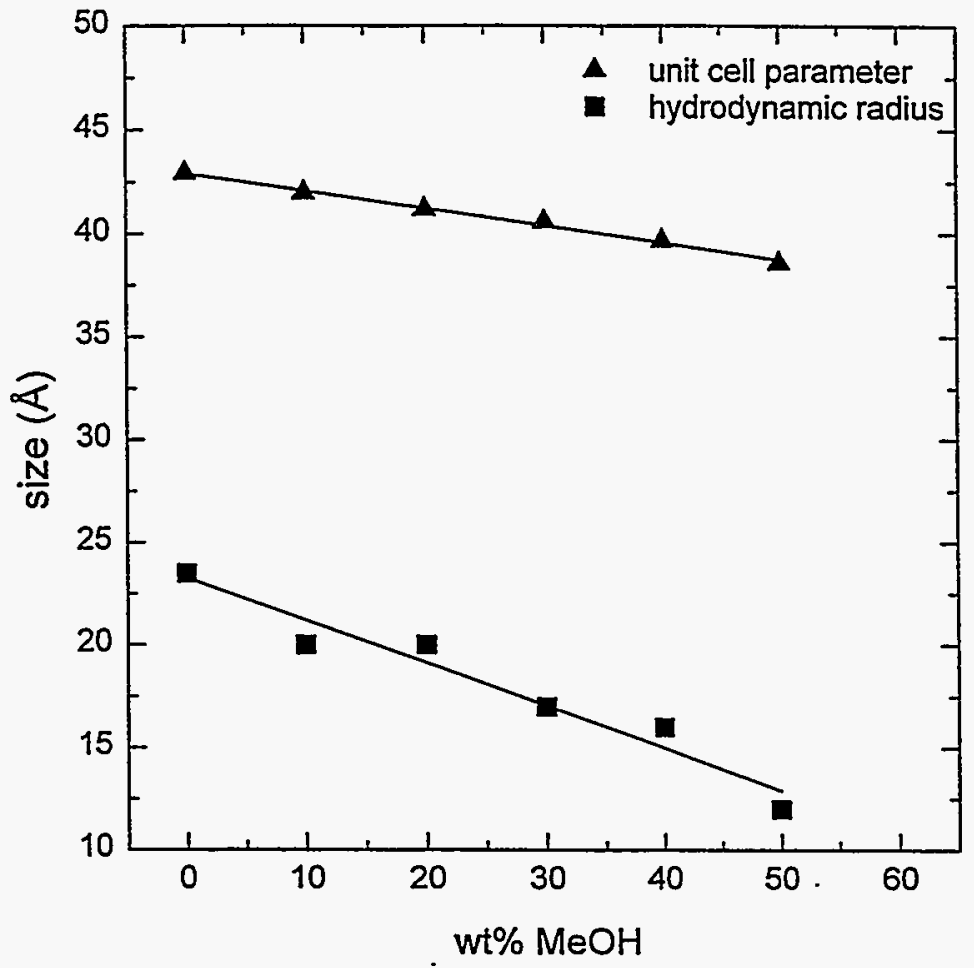

Figure 88. The cell constants of the $\mathrm{H}$ mesophases made from a water:methanol system decreases as $r$ increases as do the hydrodynamic radii of micelles in the precursor solution. The slope of the cell constant plot (da/dr) is $-8.0 \AA / 100 \%$.

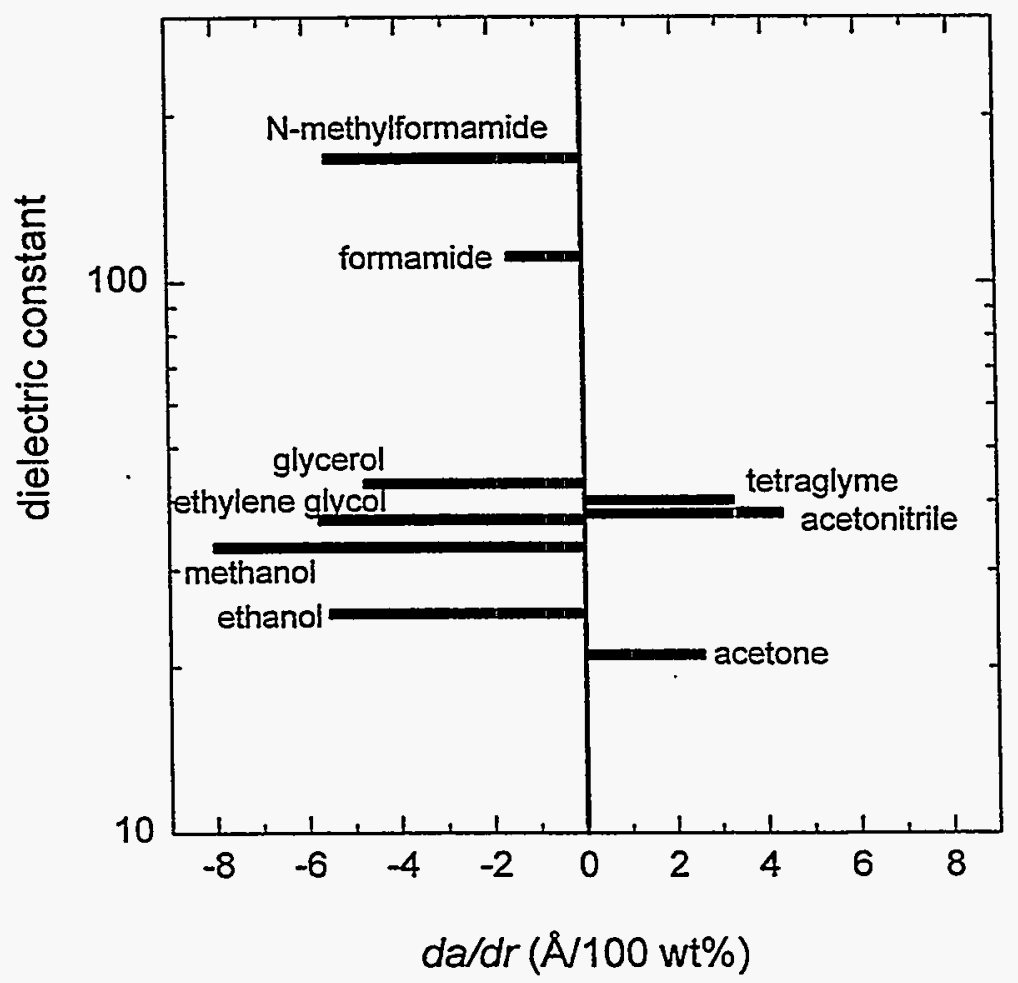

Figure 89. The graph shows the slope of graphs similar to that shown in Fig. 29 for the cosolvents indicated. A negative slope indicates the cell constant decreases with increase in $r$. 
To make heteroatomic precursors we first mixed two alkoxides in a nonaqueous environment. For example, TMOS (4.22 mmol) and aluminum sec-butoxide $(0.422 \mathrm{mmol})$ were mixed with isopropanol to form homogeneous solutions, the concentration in five solutions ranging from 1.8 to $8.6 \mathrm{M}$ silica. Water was then added to these solutions to promote hydrolysis and condensation and after aging these solutions were added to a basic aqueous micelle solution. The resulting mixtures had $r=5-25 \%$ and a constant $0.45 \mathrm{M}$ silica concentration. After 7-10 $\mathrm{s}$ an aluminum containing periodic mesophase gel resulted, Fig. 90. The $r=5 \%$ sample had the largest CSD. ${ }^{27} \mathrm{Al}$ NMR indicates that all of the aluminum in each of the five samples is 4-coordinate, Fig. 91, indicating that all the $\mathrm{Al}$ is incorporated into the silica framework. However, after calcination only $\sim 2 / 3$ of the Al is 4 coordinate, the rest is 5- or 6-coordinate and is assumed to be extraframework, which occurs commonly for mixed-metal $\mathrm{Al}: \mathrm{SiO}_{2}$ framework mesophases $[6,50,51]$.

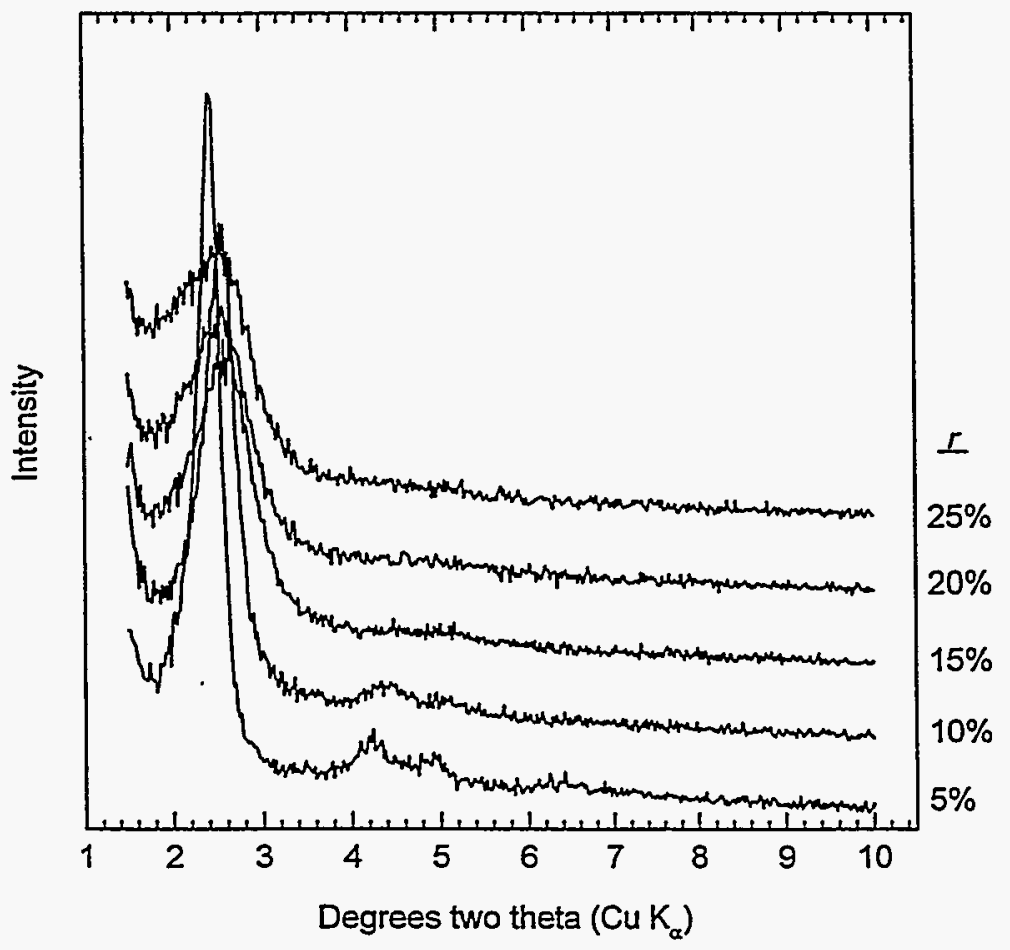

Figure 90. X-ray diffraction from samples made by premixing TMOS and $\mathrm{Al}(\mathrm{sec}-\mathrm{OBu})_{3}$ in a 10:1 molar ratio in isopropanol and then adding this solution to an aqueous alkaline micellar solution. The value of $r$ indicates the weight percent isopropanol in the final mixture. 


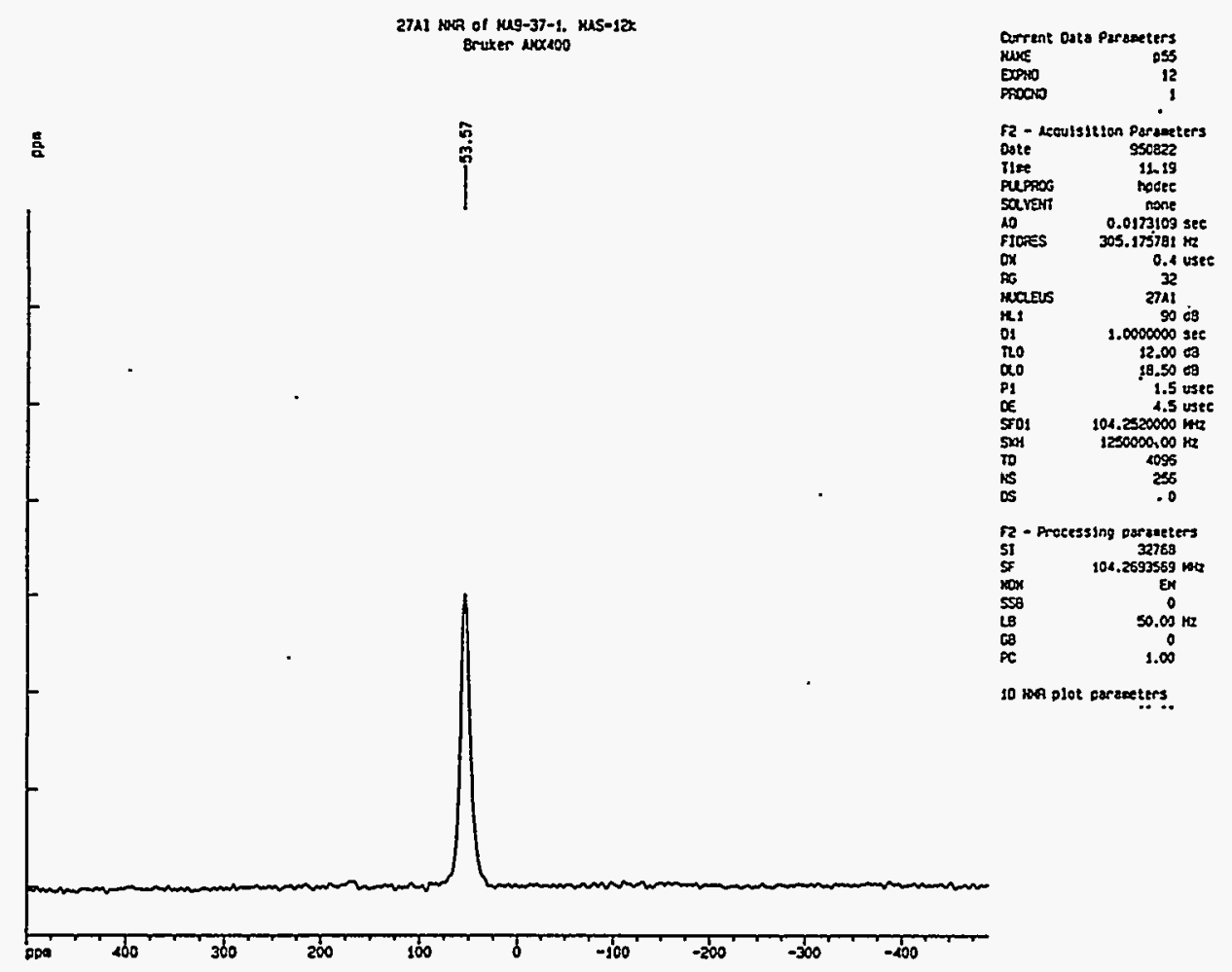

Figure $91 .{ }^{27} \mathrm{Al}$ solid state magic angle spinning $\mathrm{NMR}$ recorded on the $r=5 \%$ sample indicate all of the $\mathrm{Al}$ is four coordinate.

\section{D.6 Effect of Cosolvents on Microstructure - TMOS-derived periodic} mesophases occur as aggregate gels of submicron particles, Fig. 92. In general, the presence of cosolvents little affects the size range of grains, but can effect dispersity and grain shape. Samples made by treating neutral solutions with $\mathrm{NH}_{3}$ gas tend to exhibit particle sizes an order of magnitude smaller than for samples made with $\mathrm{NaOH}$. We examined the effects of seven cosolvents on microstructure and summarized the results in Table II.

D.7 Swelling Agents -- Under some circumstances, adding water-immiscible, low dielectric constant liquids (oils) to micellar solutions swells the micelle to produce a microemulsion (i.e. thermodynamically stable solution of surfactant, oil and solvent) [47]. The dissolved oil thus provides a means of increasing the micelle radius which might then be used to increase the pore diameter of the mesophase. This strategy is different than that already employed, where an oil is added to the reacting solution to swell the nascent mesophase [1,4]. This procedure results in $\mathrm{H}$ products that exhibit 4 or more 


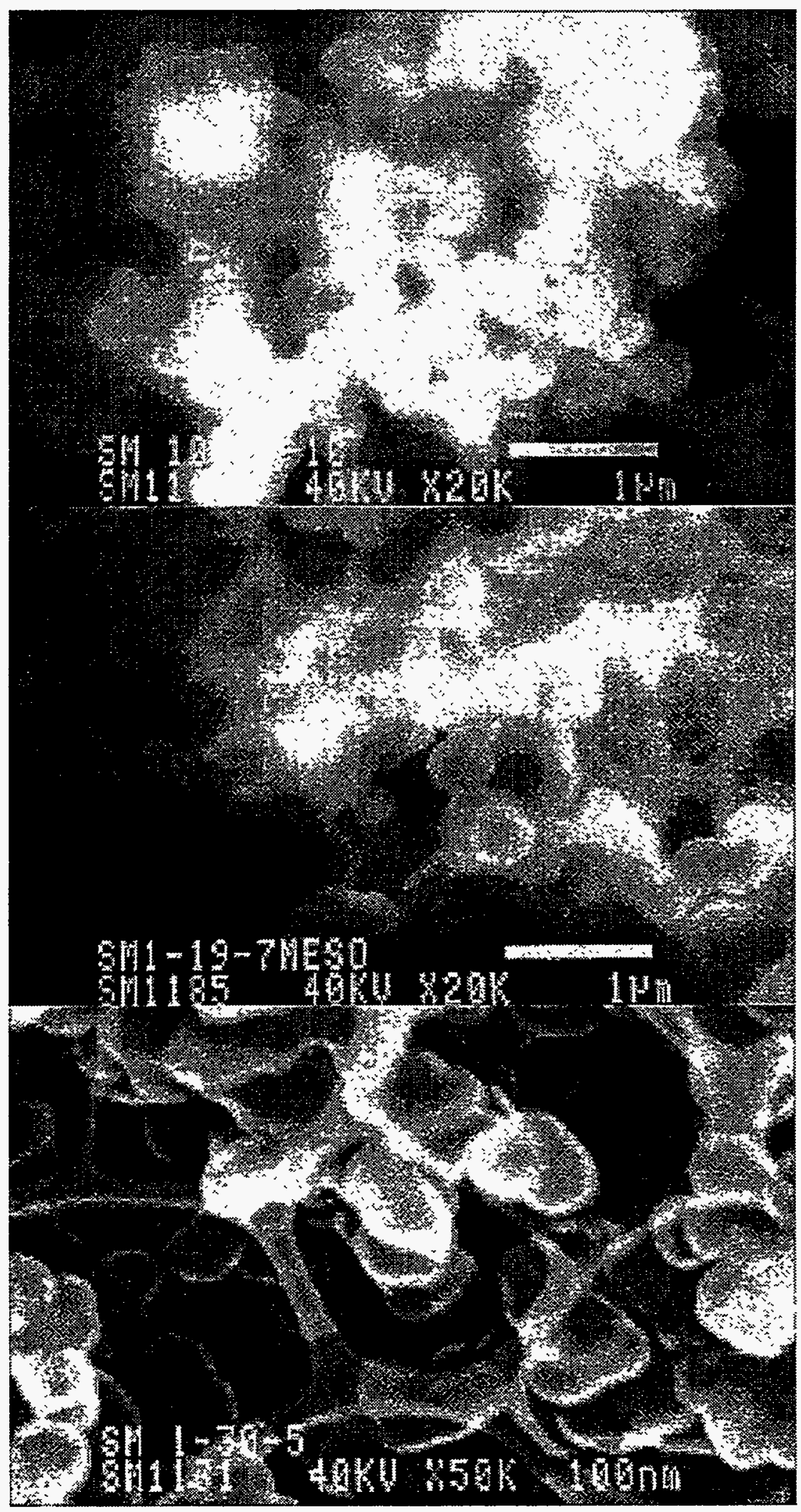

Figure 92. Top to bottom: particles grown in formamide, acetone and tetrahydrofuran. 
Table II. Effect of Cosolvents on Microstructure

\begin{tabular}{|c|c|c|c|}
\hline cosolvent & $r(\%)$ & grain size $(\mathrm{nm})$ & $\begin{array}{l}\text { grain } \\
\text { shape }\end{array}$ \\
\hline water & - & $150-500$ & $E$ \\
\hline tetrahydrofuran & 20 & $150-600$ & $\mathrm{E} / \mathrm{S}$ \\
\hline tetrhydrofuran & 10 & $150-600$ & $E / S$ \\
\hline acetone & 40 & $300-600$ & $S$ \\
\hline isopropanol & 15 & $200-750$ & $E$ \\
\hline methanol & 25 & $150-500$ & $E / S$ \\
\hline methanol $^{2}$ & 25 & $250-500$ & $E$ \\
\hline methanol & 40 & $150-700$ & $E$ \\
\hline formamide & 90 & $200-1000$ & $\mathrm{E} / \mathrm{S}$ \\
\hline formamide ${ }^{3}$ & 50 & $25-100$ & $S$ \\
\hline ethylene glycol & 50 & $10-50$ & $\mathrm{~S}$ \\
\hline
\end{tabular}

Note: E-ellipsoidal; S - spherical.

${ }^{1}$ made with a 2-butanol:CTAB ratio of 2.7:1.

${ }^{2}$ made at $10^{\circ} \mathrm{C}$ and $50^{\circ} \mathrm{C}$ with a 2-butanol:CTAB ratio of 2.7:1.

${ }^{3}$ thin film made with ammonia gas rather than $\mathrm{NaOH}$ as the catalyst.

diffraction peaks; but the solutions are inhomogeneous and invariably provide less than quantitative yields.

To make swollen mesophases we initially employed a cosolvent (e.g. methanol) in order to ensure that the silicon source completely dissolved prior to product formation. We discovered the cosolvent destabilized the microemulsion and did not allow swollen products to form. We omitted the cosolvent and added the cosurfactant 2-butanol (cosurfactant:surfactant $=0.0$ to 2.5) to the solution in an attempt to stabilize the microemulsion. Dynamic light scattering on the solutions confirms that we formed microemulsions in which the micelle radius is larger than in the absence of added oil, but that are quite polydisperse, Fig. 93. 


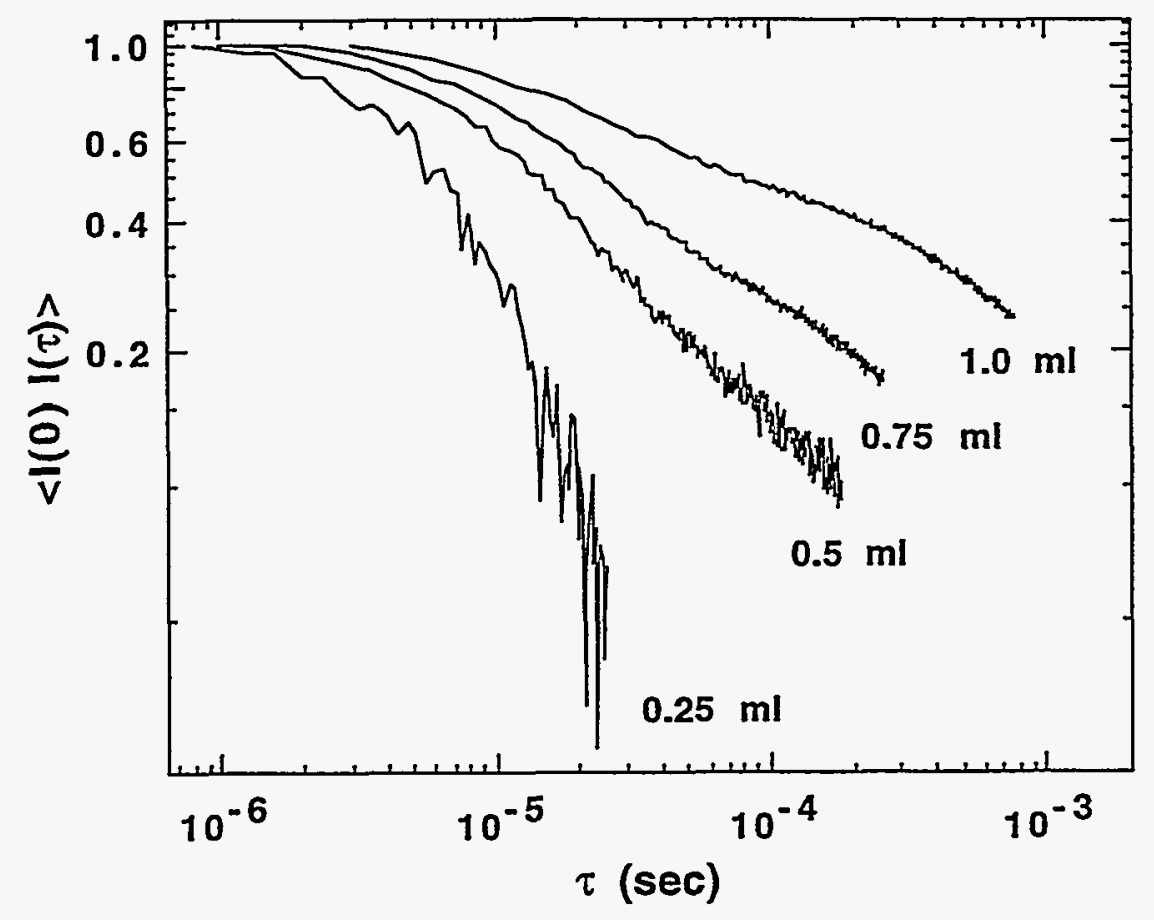

Figure 93. The nonexponential correlation intensity autocorrelation functions obtained by dynamic light scattering indicate a polydisperse ensemble of microemulsion droplets, each of which has a relaxation time that is determined by its size-dependent diffusion constant.

From these solutions, we made products with $d_{100}$ spacings larger than those made from solutions with no oil. We made $L, D$, and $A$ products. We could not unambiguously determine if the materials with one peak are hexagonal or lamellar, but the materials exhibit $d_{100}$ spacings up to $62 \AA$ (a » 72 $\AA$ ), Fig 94. Lamellar products commonly form from solutions that contain a high surfactant:silicon ratio $(>0.5)$ or that are concentrated in surfactant (8 $w t \%)$. The latter form hexagonal products in the absence of oils.

Because the hydrolysis of TMOS liberates the lower alcohol methanol, which tends to destabilize microemulsions, we eliminated all methanol from the solution by using the molecular silica sources TXOS ( $X=$ ethoxy, propoxy, butoxy). These silica sources have the advantage that they liberate higher alcohols that could actually act as cosurfactants when hydrolyzed. However, in control experiments without oils we found that these silica sources result in inhomogeneous solutions, that even in the presence of homogenizing 
agents lead to $\mathrm{D}$ or $\mathrm{A}$ products. When oil is added to the mixture the same type of products that form in the TMOS systems ( $L, D$, and $A$ ) are produced.

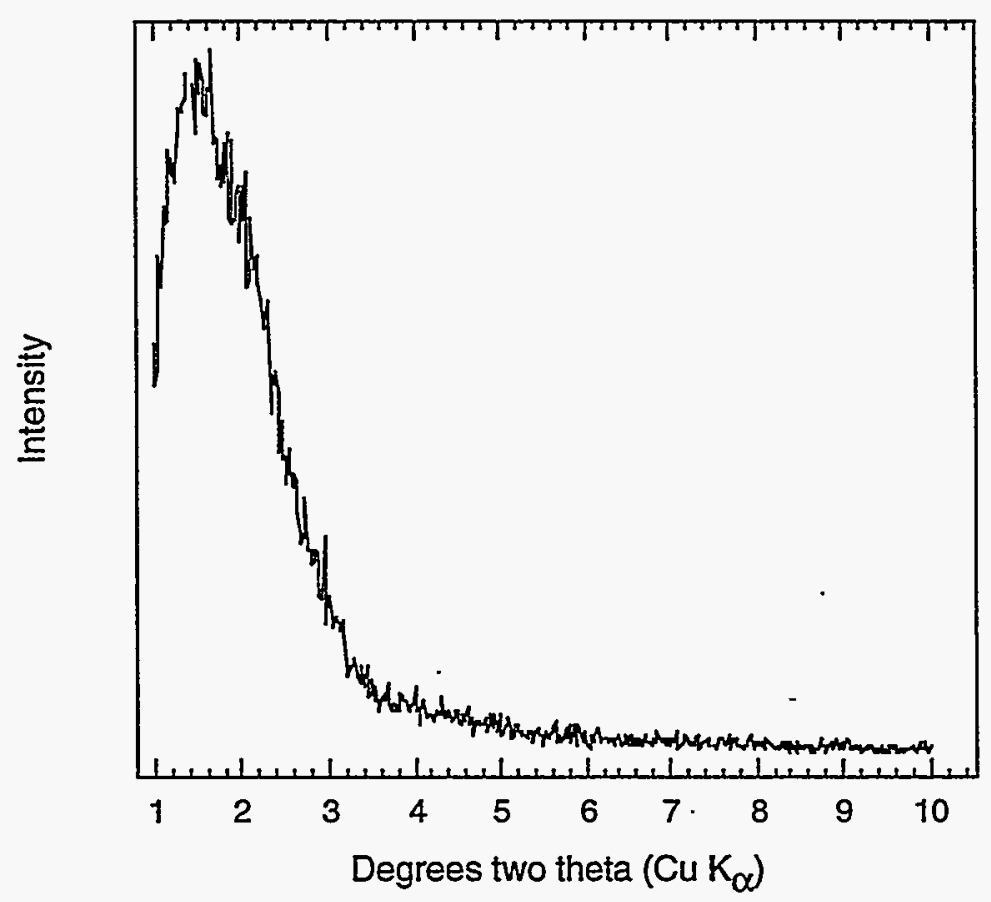

Figure 94. XRD data for a swollen lamellar mesophase with a lattice spacing of $72 \AA$.

We conclude: (1) that it is possible to form swollen mesophases ( $a$ up to -72 A) from microemulsions, but because of the polydispersity of the microemulsion it is difficult to form a regular array of pores; (2) by adding a small amount of oil to a concentrated surfactant- silicon solution (e.g. CTAB:oil: $\left.\mathrm{H}_{2} \mathrm{O}: \mathrm{Si}=0.16: 0.44: 41: 1\right)$, $\mathrm{L}$ phases form at molar ratios where $\mathrm{H}$ phases would form in the absence of oil; (3) the molecular structure of the swelling agent has little or no effect on the periodicity or final pore size of the swollen product; and (4) high concentrations of cosolvents can destabilize microemulsions and prevent the formation of swollen mesophases.

Finally, we found that a vigorously stirred two-phase mixture of water and the volatile liquid 1,1,2- trichloroethane leads to periodic swollen $\mathrm{H}$ and cubic (C) products. To accomplish this a 50:50 mixture of methanol and 1,1,2trichloroethane was added in increasing amounts to the standard TMOS 
preparation. This resulted in swollen hexagonal products with cell constants up to $\sim 54 \AA$ (vs $44 \AA$ in pure water) and a cubic phase with a cell constant of $\sim 102 \AA$ as shown in Fig. 95. Owing to the large excess of swelling agent in this two-phase mixture, it is difficult to determine whether the 1,1,2trichloroethane dissolves in the micelles before the product forms or whether it dissolves in the surfactant core of the product after it forms. Our inability to form ordered products from microemulsions and the success of previous workers who dissolved oil in previously formed mesophases, indicates that the latter is more likely.

D.8 Added Salt - It is believed that the initial step in the formation of periodic mesophases is the displacement of surfactant counterions by multidentate silicate oligomers [4]. It should thus be possible to alter the reaction kinetics and potentially inhibit mesophase formation by using anions that strongly bond to the surfactant head groups. We have examined the effect of mono-, di-, and trivalent anions that are uni- or multidentate to determine their effect on mesophase formation. We used the salts $\mathrm{KI}, \mathrm{K}_{2} \mathrm{SO}_{4}$, $\mathrm{NaCl}, \mathrm{NaH}_{2} \mathrm{PO}_{4}$ (titrated to $\mathrm{pH} 13$ with $\mathrm{NaOH}$ ) and $\mathrm{K}_{2} \mathrm{C}_{2} \mathrm{O}_{4}$ in concentrations up to $2.5 \mathrm{M}$.

In general, at anion concentrations less than $0.5 \mathrm{M}$ (salt:silica < 1.2:1) the added salt had no measurable effect on cell constants or gelation time. In solutions that contain $\mathrm{Cl}^{-}, \mathrm{I}^{-}, \mathrm{SO}_{4}{ }^{2-}$ and $\mathrm{PO}_{4}{ }^{3-}$ in concentrations up to $2.0 \mathrm{M}$, we found that the ions increase the gel time by less than $\sim 30 \%$ (oxalate has a more pronounced effect, as discussed below). The gel time increases in the order $\mathrm{I}^{-} \approx \mathrm{Cl}^{-}<\mathrm{SO}_{4}^{2-}<\mathrm{PO}_{4}^{3-}<\mathrm{C}_{2} \mathrm{O}_{4}^{2-}<$ silicate.

The effect of oxalate ion on gel kinetics is independent of concentration until the oxalate:Si ratio exceeds 1.2 ( $0.5 \mathrm{M}$ salt). At ratios greater than 1.2, there is a linear dependence of gel time on oxalate ion concentration, Fig. 96. This behavior implies that oxalate ions are able to effectively screen the silicate ions from the binding sites at the surfactant head 


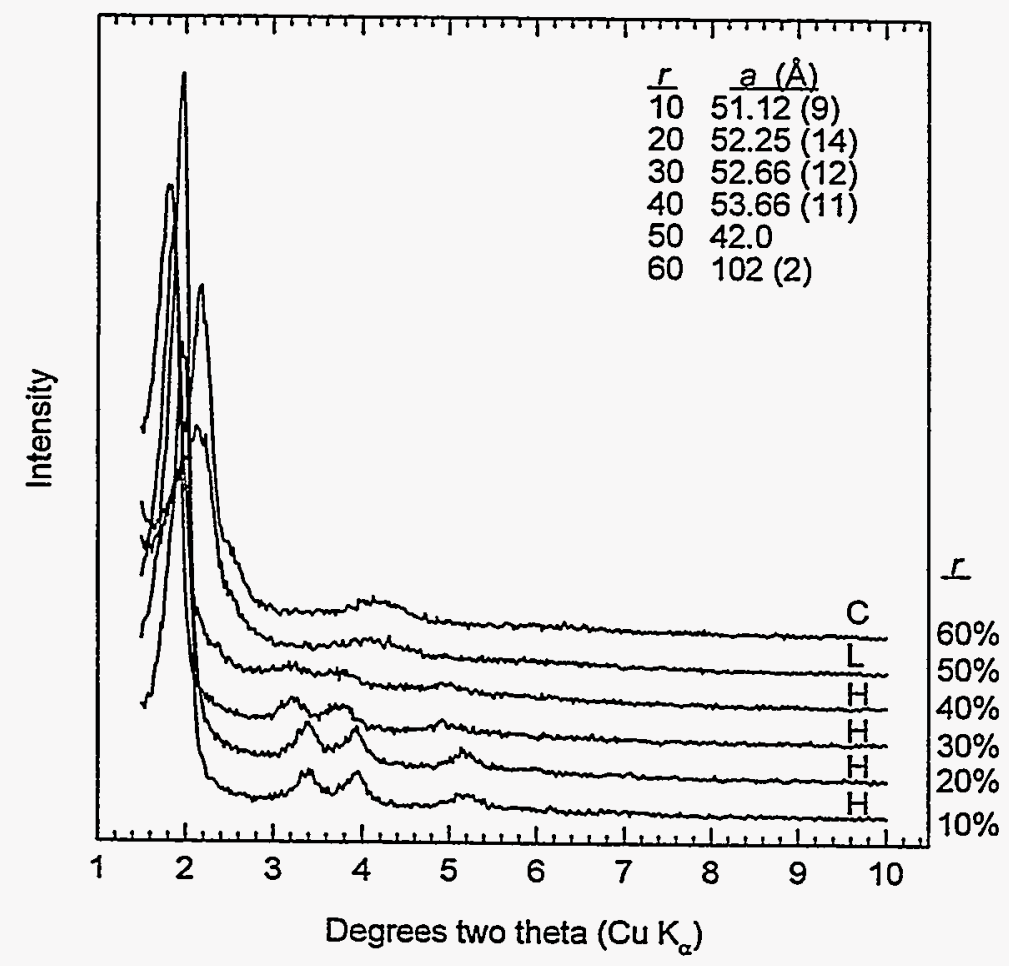

Figure 95. $X$-ray diffraction patterns for a 2-phase water:cosolvent (cosolvent $=50: 50$ methanol:1,1,2,-trichloroethane) system show the evolution from to $C$ as $r$ increases. The cell constant is $\sim 16$ to $\sim 22 \%$ larger for the $H$ phases than for $H$ phases made in pure water.

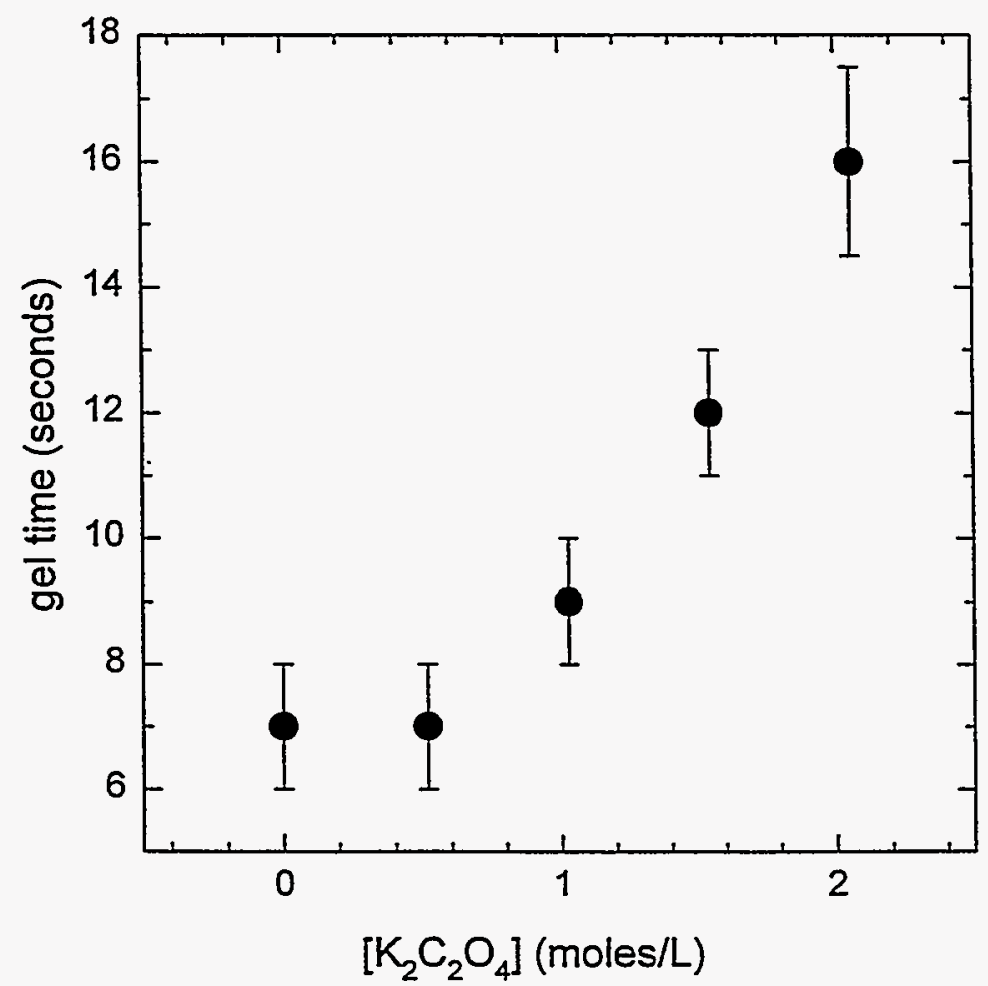

Figure 96. The gelation time increases linearly with $\mathrm{K}_{2} \mathrm{C}_{2} \mathrm{O}_{4}$ concentration at high concentrations of salt. 
groups when present in concentrations greater than the silicate species.

It is interesting to note that the oxalate: $\mathrm{CTA}^{+}$ratio in the $0.5 \mathrm{M}$ salt solution is $\sim 9: 1$. This means that there is about 18 times more oxalate ions in solution than would be necessary to completely balance the charge on the surfactant head groups. Clearly the oxalate ions can completely encrust the surfactant assemblies and significantly increase the ionic strength before they effect gel kinetics.

Increasing the oxalate concentration also increases the cell constant from $\sim 44$ to $\sim 49 \AA$ and decreases the CSD, Fig. 97. The increase in lattice parameter is consistent with previous observations that addition of neutral electrolyte to solutions of ionic surfactants in aqueous solutions cause an increase in aggregation number and volume of the surfactant assemblies [47].

The calcined material made from the $0.5 \mathrm{M}$ solution exhibits a surface area $\left(820 \mathrm{~m}^{2} / \mathrm{g}\right)$, pore volume $(0.58 \mathrm{cc} / \mathrm{g})$, and a pore radius $(28.1 \AA)$ very similar to materials synthesized in the absence of added salt. The wall thickness is $\sim 10 \AA$, which is comparable to samples made with no added salt.

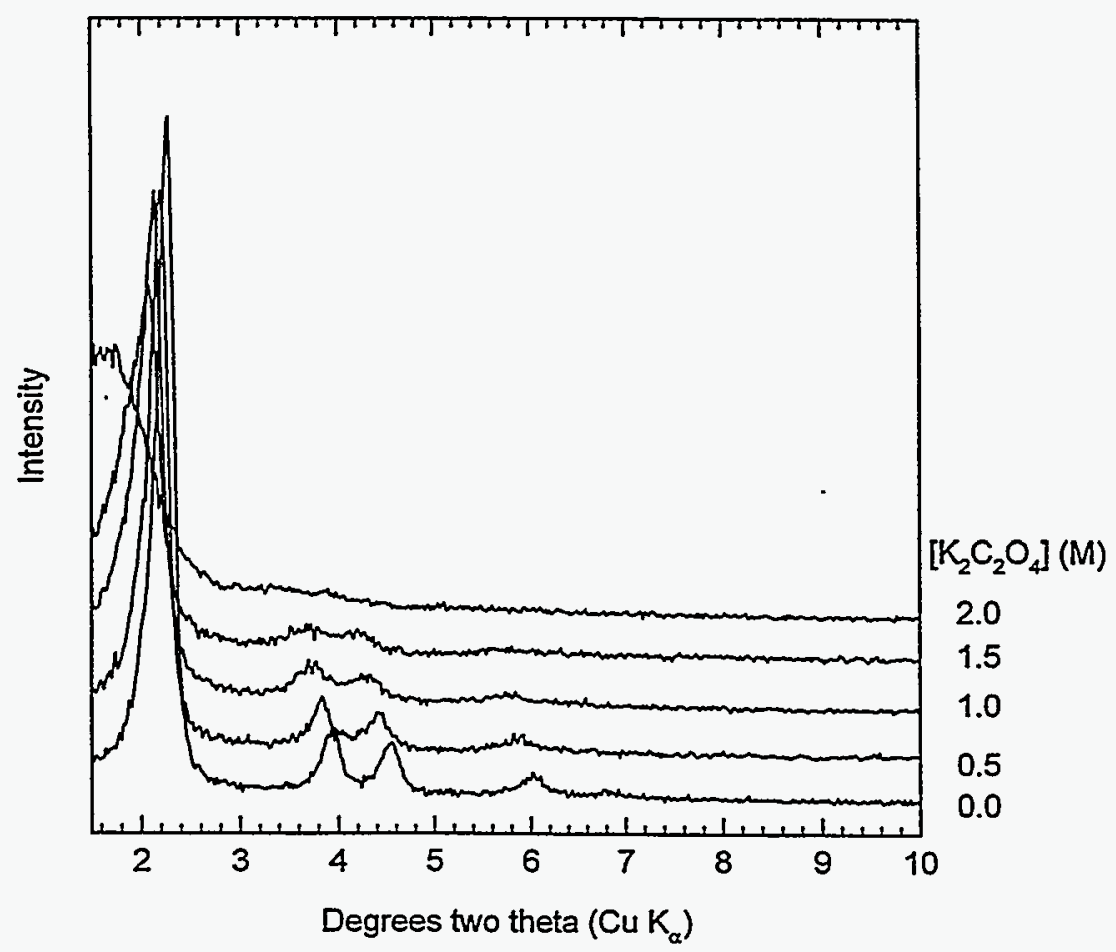

Figure 97. $X$-ray diffraction shows that the cell constant increases and the long range order decreases for $\mathrm{H}$ mesophases made with high $\mathrm{K}_{2} \mathrm{C}_{2} \mathrm{O}_{4}$ concentrations. 


\section{E. Conclusions}

Cosolvents, oils, and salt can be useful agents in controlling the synthesis of mesoporous silica. For example, cosolvents change the aggregate radius and decrease the degree of aggregation (cmic) in micellar solutions, and they change the pore radius and decrease coherent scattering domain size in mesophases. Oils swell micelles to form polydisperse microemulsions that increase the pore diameter and decrease the order parameter of mesophases. Salts tend to increase the aggregation number of micelles, and they increase the pore diameter of mesophases. These similarities illustrate that mesophase formation is affected by thermodynamics that governs the precursor micelle solutions, and that by altering the thermodynamics, fine control over the physical properties of mesophases can be exercised in the synthesis stage.

\section{Acknowledgments}

We thank R. Assink (SNL) and S. Prabakar (UNM) for collecting the NMR data. This work was funded by the United States Department of Energy under Contract No. DE-AC04-94AL85000. 


\section{References}

[1]. Beck, J. S.; Vartuli, J. C.; Roth, W. J.; Leonowicz, M. E.; Kresge, C. T.; Schmitt, K. D.; Chu, C. T-W.; Olson, K. H.; Sheppard, E. W.; McCullen, S. B.; Higgins, J. B.; Schlenker, J. L. J. Am. Chem. Soc. 1992, 114, 10834-10843.

[2]. Kresge, C. T.; Leonowicz, M. E.; Roth, W. J.; Vartuli, J. C.; Beck, J. S. Nature, 1992, 359, 710712.

[3]. Huo, Q.; Margolese, D. I.; Ciesla, U.; Feng, P.; Gier, T. E.; Sieger, P.; Leon, R.; Petroff, P. M.; Schuth, F.; Stucky, G. D. Nature, 1994, 24, 317-321.

[4]. Huo, Q.; Margolese, D. I.; Ciesla, U.; Demuth, D. G.; Feng, P.; Gier, T. E.; Sieger, P.; Firouzi, A.; Chmelka, B. F.; Schuth, F.; Stucky, G. D. Chem. Mater. 1994, 6, 1176-1191.

[5]. Tanev, P. T.; Pinnavaia, T. J. Science, 1995, 267, 865-867.

[6]. Chen, C-Y.; Li, H-X.; Davis, M. E. Micropor. Mater. 1993, 2, 17-26.

[7]. Kloetstra, K. R.; van Bekkum, H. J. Chem. Res, Synop. 1995, 1, 26-27.

[8]. Armengol, E.; Maria, L.; Corma, A.; Garcia, G.; Navarro, M. T. J. Chem. Soc., Chem. Commun. 1995, 5, 519-520.

[9]. Kloetstra, K. R.; van Bekkum, H. J. Chem. Soc., Chem. Commun. 1995, 10, 1005-1006. [10]. Corma, A.; Fornes, V.; Navarro, M. T.; Perez-Pariente, J. J. Catal. 1994, 148, 569.

[11]. Corma, A.; Navarro, M. T.; Perez-Pariente, J. J. Chem. Soc., Chem. Commun. 1994, 147.

[12]. Tanev, P. T.; Chibwe, M.; Pinnavaia, T. J. Nature 1994, 368, 321.

[13]. Schuth, F. Ber. Bunsen-Ges. 1995, 99(11), 1306-1315.

[14]. Corma, A.; Fornes, V.; Navarro, M. T.; Perez-Pariente, J. J. Catal. 1994, 148, 569.

[15]. Corma, A.; Martinez, A.; Martinez-Soria, V.; Monton, J. B. J. Catal. 1995, 153, 25.

[16]. Kresge, C. T.; Marler, D. O.; Rav, G. S.; Rose, B. H. U.S. Patent 5366 945, 1994.

[17]. Kozhevnikov, I. V.; Sinnema, A.; Jansen, R. J. J., Pamin, K.; van Bekkum, J. Catal. Lett. $1995,30,241-252$.

[18]. Wu, C-G.; Bien, T. Science 1994, 264, 1757-1759.

[19]. Wu, C-G.; Bien, T. Science 1994, 266, 1013.

[20]. Olson, D. H.; Stucky, G. D.; Vartuli, J. C. U. S. Patent 5364 797, 1994.

[21]. Berne, B.; Pecora, R. Dynamic Light Scattering; Wiley: New York, 1976.

[22]. Cogan, J. D.; Setterstrom, C. A. Chem. \& Eng. News 1946, 24, 2499.

[23]. Light Scattering in Liquids and Macromolecular Solutions DeGiorgio, V.; Corti, M.; Giglio, M.; Eds.; Plenum: New York, 1980.

[24]. Nicoli, D. F.; Ciccolello, R.; Briggs, J.; Dawson, D. R.; Offen, H. W.; Romstead, L.; Bunton, C. A. in Scattering Techniques Applied to Supramolecular and Nonequilibrium Systems 
Chen, S.; Chu, B.; Nossal, R.; Eds.; NATO Advanced Study Institute Series, Series B: Physics, Plenum: New York, Vol. 73, 1981; p 363.

[25]. The instrumental peak breadth (FWHM), as determined by using an Ag-boehmite standard in conjunction with data from a NIST Si standard, is $D(2 q)_{\text {inst }} \gg 0.10^{\circ}$ for the 100 Bragg reflection.

[26]. This was determined by observing the optical behavior of the solutions. For $t_{\text {solubility }}>t_{\text {gel }}$ the solution remains translucent until $t_{\text {gel. }}$. For $t_{\text {solubility }}<t_{\text {gel }}$ the solution is initially translucent and then turns transparent before $t_{\text {gel }}$.

[27]. Brinker, C. J.; Scherer, G. W. Sol-Gel Science Acedemic: New York, 1990; Chapter 13.

[28]. The surfactants are $99+\%$ pure. The primary impurities are assumed to be aliphatic and hydrophobic, and they most likely reside inside at the hydrophobic interior of the micelle. Such species are more soluble in alcohol than in water. Partitioning of these species into the continuous phase could increase the dispersity of micelles. This could lead to an increase in CSD size and may contribute to the increase in CSD size.

[29]. Maschmeyer, T.; Rey, F.; Sankar, G.; Thomas, J. M. Nature 1995, 378, $159-162$.

[30]. Ogawa, M. J. Am. Chem. Soc. 1994,116, 7941-7942.

[31]. Yang, H.; Kuperman, A.; Coombs, N.; Mamiche-Afara, S.; Ozin, G. A. Nature 1996, 379, 703-705 (1996).

[32]. Bontha, J. R.; Kim, A. Y.; Liu, J. Mater. Res. Soc. Symp. Proc. P: Microporous and Macroporous Materials, April 8-12, San Fracisco, CA, 1996.

[33]. Anderson, M. T.; Martin, J. E.; Odinek, J. G.; Newcomer, P. P., submitted to Chemistry of Materials.

[34]. Ricco, A. J.; Frye, G. C.; Martin, S. J. Langmuir 1989, 5, 273-276

[35]. The Fractal Approach to Heterogeneous Chemistry; Avnir, Ed.; Wiley: New York, 1989; 131.

[36]. Mandelbrot, B. B. The Fractal Geometry of Nature; Freeman: New York, 1977.

[37]. Anderson, M. T.; Martin, J. E.; Odinek, J. G.; Newcomer, P. P. to be submitted to Journal of Physical Chemistry.

[38]. Anderson, M. T.; Martin, J. E.; Odinek, J. G.; Newcomer, P. P., Wilcoxon, P. J. Mater. Res. Symp. Proc., this volume.

[39]. Brinker, C. J.; Scherer, G. W. In Sol-Gel Science; Academic: New York, 1990; Chapter 9.

[40]. It is well know that aerogels have an 'open' porosity, so that the void space percolates.

However, a small sphere diffusing through the void space will see a broad distribution of pore dimensions, and will find many narrow apertures through which it cannot pass. A very small sphere will be able to percolate through the pore volume, but for a given aerogel there will be a critical sphere size $R_{c}$ that marks the threshold of percolation. Larger 
spheres will be localized, with a localization length that decreases as the sphere size increases.

[41]. We do not believe this is merely a double layer effect, as the Debye screening length depends only on the ionic strength and adding salt does not induce phase separation.

[42]. Martin, J. E.; Adolf, D. Annu. Rev. Phys. Chem. 1991, 42, 311-319.

[43]. Martin, J. E.; Wilcoxon, J. P. Phys. Rev. A 1989, 39, 252-258.

[44]. Lin, W.; Chen, J.; Sun, Y.; Pang, W. J. Chem. Soc., Chem. Commun. 1995, 2367.

[45]. Martin, J. E.; Wilcoxon, J. P.; Schaefer, D.; Odinek, J. Phys. Reo. A, 1990, 41(8), 4379-4391.

[46]. Martin, J. E.; Odinek, J. J. Coll. and Interfac. Science 1992, 154(2), 461-479..

[47]. see for example (a). Rosen, M. J. in Surfactants and Interfacial Phenomena; Wiley: New York, 1989; Chapters 3 and 4. (b). Meyers, D. in Surfactant Science and Technology; VCH: New York, 1992; Chapters 3 and 4.

[48]. Wärnheim, T.; Jönsson, A. J. Coll. Intfac. Sci. 1988, 125(2), 627-633.

[49]. Auvray, X; Petipas, C.; Anthore, R.; Rico, I.; Lattes, A. J. Phys. Chem. 1989, 93, 74587464.

[50]. Kim, A. Y.; Lui, J.; Virden, J. W.; Bunker, B. Mater. Res. Soc. Symp. Proc.; Komarneni, S.; Smith, D.; Beck, J. S. Eds.; MRS Symposium Series 371; Materials Research Society: Pittsburgh, PA, 1995; pp 105-110.

[51]. Schmidt, R.; Akporiaye, D.; Stöcker, M.; Ellestad, O. E.; J. Chem. Soc., Chem. Commun. 1994, 1493. 


\section{DISTRIBUTION:}

$\begin{array}{lll}1 & \text { MS-1436 } & \text { C. E. Meyers, 1007 } \\ 1 & \text { MS-1427 } & \text { P. L. Mattern, 1100 } \\ 5 & \text { MS-1421 } & \text { J. E. Martin, 1152 } \\ 1 & \text { MS-1421 } & \text { P. Newcomer, 1152 } \\ 1 & \text { MS-1421 } & \text { G. Samara, 1152 } \\ 5 & \text { MS-1349 } & \text { M. T. Anderson, 1815 } \\ 1 & \text { MS-9018 } & \text { Central Tech Files, 8523-2 } \\ 5 & \text { MS-0899 } & \text { Technical Library, 4414 } \\ 2 & \text { MS-0619 } & \text { Review and Approval Desk, 12630 } \\ 44 & & \text { For DOE/OSTI-UC404 }\end{array}$

